Space-time monitoring of tropical forest changes using observations from multiple satellites

Eliakim Hamunyela 


\section{Thesis committee}

\section{Promotor}

Prof. Dr M. Herold

Professor of Geo-information Science and Remote Sensing

Wageningen University \& Research

\section{Co-promotor}

Dr J.P. Verbesselt

Associate professor, Laboratory of Geo-information Science and Remote

Sensing Wageningen University \& Research

\section{Other members}

Prof. Dr W. Peters, Wageningen University \& Research

Prof. Dr E.J. Pebesma, Westfälische Wilhelms-Universitt Münster, Germany Dr M. Mahecha, Max Planck Institute for Biogeochemistry, Jena, Germany Dr S. Lhermitte, Delft University of Technology, Delft

This research was conducted under the auspices of the C.T. de Wit Graduate School of Production Ecology \& Resource Conservation (PE\&RC) 


\title{
Space-time monitoring of tropical forest changes using observations from multiple satellites
}

\author{
Eliakim Hamunyela
}

\section{Thesis}

submitted in fulfilment of the requirements for the degree of doctor at Wageningen University

by the authority of the Rector Magnificus

Prof. Dr A.P.J. Mol, in the presence of the

Thesis Committee appointed by the Academic Board to be defended in public

on Wednesday 6 September 2017 at 4 p.m. in the Aula. 
Eliakim Hamunyela

Space-time monitoring of tropical forest changes using observations from multiple satellites

204 pages

$\mathrm{PhD}$ thesis, Wageningen University, Wageningen, the Netherlands (2017)

With references, with summary in English

ISBN 978-94-6343-640-3

DOI: $10.18174 / 420048$ 
Dedicated to my father: Kornelius Namukwiyu. 



\section{Contents}

Chapter 1 Introduction

Page

1

Chapter 2 Using spatial context to improve early detection of deforestation from Landsat time series

Chapter 3 Monitoring deforestation at sub-annual scales as extreme events in Landsat data cubes

Chapter 4 Using space-time features to improve detection of forest disturbances from Landsat time series

Chapter 5 Combining Sentinel-2 and Landsat time series for small-scale forest change monitoring

Chapter 6 Improving near-real time deforestation monitoring in tropical dry forests by combining dense Sentinel1 time series with Landsat and ALOS-2 PALSAR-2

Chapter $7 \quad$ Synthesis

References

Summary

Acknowledgements

Publications

Short biography

Education and training statement 



\section{Chapter}

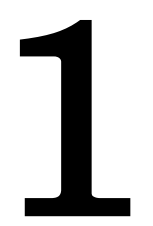

Introduction 


\section{$1.1 \quad$ Background}

Humanity is highly depended on forests for a number of products (e.g. timber) and services (e.g. mitigation of global warming), but humanity has been deforesting and degrading forests at alarming rates in recent years. When forests are deforested or degraded (Fearnside 1996; Fearnside 2000), they contribute immensely to greenhouse gas (GHG) emissions (Pan et al. 2011; Arneth et al. 2017; Le Quéré et al. 2009). Increased GHG concentrations in the atmosphere enhance global warming (Heimann \& Reichstein 2008), leading to frequent occurrence of extreme climatic conditions i.e. heatwaves and droughts (Coumou \& Rahmstorf 2012; Meehl \& Tebaldi 2004). These extreme climatic conditions weaken the capacity for forest ecosystems to act as sinks for carbon (Reichstein et al. 2013). Moreover, extreme climatic conditions reduce food production (Ciais et al. 2005; Lobell et al. 2012) and they ultimately undermine the capacity for forest ecosystems to regulate the climate system (Bonan 2008). Deforestation and forest degradation also contribute to biodiversity loss (Newbold et al. 2015), and reduce the capacity for forest ecosystems to prevent soil erosion and sustain hydrological cycles (Luyssaert et al., 2008). Despite these negative impacts, humanity is still deforesting and degrading forests in many parts of the globe (Hansen et al. 2013), with tropical areas being the hotspot for forest loss (FAO 2015).

Forests are deforested and degraded mainly to expand agricultural land (De Sy et al. 2015), but also for timber and to expand mining activities (Asner et al. 2013). Some deforestation and forest degradation activities are illegal. It is estimated that up to $10 \%$ of globally traded timber is illegally harvested (Seneca Creek Associates 2014), thus indicating that some people derive important economic value from illegally harvested timber. With human population increasing by 2 billion people in the next three decades (Cohen 2003), forest loss is expected to accelerate, triggering further loss of biodiversity (Jenkins 2007). Losing more forest in the next decades will further diminish the capacity for forests to provide goods and services to humanity.

Several strategies aimed at reducing forest loss have been suggested recently. One of these strategies is land sparing which refers to interventions which seek to minimise the demand for new agricultural land by increasing yield from existing agricultural land (Lamb et al. 2016; Green et al. 2005). Since agriculture is the main driver of forest loss (De Sy et al. 2015), increasing yield on existing agricultural lands can be viewed as an important approach to reduce agriculture-driven forest loss (Carter et al. 2015; Lamb et al. 2016; Green et al. 2005). The REDD+ (Reducing deforestation and forest degradation) is another recent strategy that has been proposed to protect forest ecosystems. This strategy aims to reward developing countries financially for protecting and enhancing forest management (UNFCCC 2009). 
When implemented correctly, the REDD+ mechanism can reduce forest loss by creating alternative incomes for local communities who live in forest areas. Land sparing and REDD+ can therefore be viewed as synergic and complementary approaches for reducing forest loss.

In a broader perspective, implementing land sparing and REDD+ strategies has potential to reduce forest loss significantly by decreasing the demand for new agricultural land and increasing the conservation of forest ecosystems. However, stopping forest loss caused by illegal forest disturbances is likely to be a major challenge especially in areas where forest are deforested and degraded illegally for commercial interests (e.g. selling timber and mining minerals).

To fully protect forest ecosystems, additional measures, other than land sparing and REDD+ mechanism, are therefore needed. Enacting and reenforcing forest protection laws are a critical measure needed to prevent illegal forest clearings (Nepstad et al. 2008), but policing large forest areas using traditional approaches can be expensive and challenging. To re-enforce forest protection laws efficiently, timely, reliable and location-specific information on new forest disturbances is needed.

Frequent and large-area forest mapping and monitoring using satellite observations can provide timely and cost-effective information about new forest disturbances (Reiche et al. 2016). This approach was instrumental in helping Brazil to reduce illegal forest clearings in recent years (Nepstad et al. 2014). However, existing forest monitoring systems still have major weaknesses. More specifically, persistent cloud cover and strong seasonal variations in photosynthetic activity and canopy water content of the forest reduce the capacity for forest monitoring systems to detect new disturbances accurately and timely. Persistent cloud cover is particularly a major challenge in the humid tropical areas, whereas strong forest seasonal dynamics are a major hindrance to timely detection of forest disturbances in the dry tropical areas. Persistent cloud cover can be addressed by using observations from multiple satellite sensors (Reiche, de Bruin, et al. 2015), but satellite sensors often have inter-sensor differences which make integration of observations from multiple sensors challenging. Furthermore, detecting small-scale and low-magnitude forest disturbances accurately and timely remains overly challenging, especially in complex forest landscapes. This is because existing forest monitoring systems use coarse spatial resolution satellite data or because the forest change detection methods are not able to detect lowmagnitude disturbances without amplifying the commission error. Currently, timely detection of forest disturbances is inherently accompanied by many false detections (Reiche, de Bruin, et al. 2015; Zhu et al. 2012), thus making the forest change alerting system unreliable. For a forest monitoring system to be reliable, it should generate alerts which are spatially and temporally accurate. To generate spatially and temporally accurate alerts, the change 
detection algorithm should simultaneously be able to (i) account for seasonality, (ii) have capacity to detect small-scale and low-magnitude forest disturbances timely while rejecting false detections, and (ii) should be able to ingest observations from multiple medium spatial resolution satellite sensors to overcome the problem of persistent cloud cover and to increase the temporal details. Unfortunately, existing change detection approaches do not have a combination of all these capabilities. This thesis fills this gap by developing a space-time forest change detection framework for monitoring forest disturbances in humid and dry tropical forests using observations from multiple satellites.

The remaining part of this chapter is structured as follows. Section 1.2 provides a brief overview of how satellite-based forest monitoring has evolved over the years. Current challenges facing near real-time satellite-based forest monitoring, including previous effort to address them, are discussed in Section 1.3. Section 1.4 highlights the limitations of the existing forest change monitoring approaches. Section 1.5 provides the overview of how the challenges facing satellite-based forest change monitoring can be addressed. The research objectives of this thesis are presented in Section 1.6, and the structure of this thesis is discussed in Section 1.7.

\subsection{Evolution of satellite-based forest monitoring}

Forest change monitoring using satellite data has evolved significantly over the years. Early work on satellite-based forest monitoring focused mainly on mapping the extent of the forest areas and other land cover types (Defries et al. 2000; Hansen et al. 2000). These mapping efforts produced several global land-cover maps, with satellite data from Pathfinder Advanced Very High Resolution Radiometer (AVHRR) sensors being the primary source for global land cover maps. AVHRR data, however, have a coarse spatial resolution $(1 \mathrm{~km})$, and are rarely able to capture small-scale land-cover dynamics. Although these land-cover maps did not provide reliable information on forest cover change, they provided critical information about the extent of global forest ecosystems. The arrival of the moderate resolution imaging spectroradiometer (MODIS) on-board of Terra and Aqua satellites in 2000 , with a free and open access data policy, provided new opportunities to produce global land-cover maps with an improved spatial resolution $(250 \mathrm{~m}-$ $500 \mathrm{~m}$ ). Our understanding of the extent for forest ecosystems worldwide was further improved when a global vegetation continuous fields (VCF) tree cover product from MODIS data (Hansen et al. 2003) became available. In subsequent years, there were other efforts to improve global tree cover products. For example, a Landsat-based VCF product with $30 \mathrm{~m}$ spatial resolution was produced by rescaling the $250 \mathrm{~m}$ MODIS VCF tree cover product using 2000 and 2005 Landsat data (Sexton et al. 2013). These VCF 
products have been used to identify areas where the forest has been cleared (Hansen \& Defries 2004), but for accounting purpose only. The extent of the global tree cover was mapped elaborately after Landsat data archives become freely accessible (Hansen et al. 2013). To date, tree cover maps remain important inputs for forest change detection (Hansen et al. 2016; Hansen \& Loveland 2012; Devries, Verbesselt, et al. 2015).

The temporal scale at which forest changes are mapped using satellite observations has also changed drastically over the years, shifting from decadal (Achard et al. 2014) and annual (Souza, Jr et al. 2013; Griffiths et al. 2012; Kennedy et al. 2010; Devries, Verbesselt, et al. 2015) to sub-annual scales in recent years (Reiche, de Bruin, et al. 2015; Xin et al. 2013; Zhu et al. 2012; Shimabukuro et al. 2006).

Forest change monitoring shifted to sub-annual scales in order to detect forest disturbances in near real-time. In literature, there is not yet a clear-cut definition of what constitutes near real-time forest change monitoring. However, it broadly refers to a monitoring scenario whereby forest disturbances are detected once a new image becomes available (Diniz et al. 2015; Verbesselt et al. 2012; Shimabukuro et al. 2006; Hammer et al. 2014) in order to provide timely information about new disturbances. Timely information about new forest disturbances became critically important in recent years to support initiatives which aim to reduce illegal forest clearings.

The shift to sub-annual forest monitoring was triggered mainly by availability of frequent, free and open access coarse resolution data from MODIS. Although MODIS data have been used widely for forest change monitoring (Setiawan et al. 2016; Shimabukuro et al. 2006; Hammer et al. 2014), it is well recognised that they are too coarse (low spatial details) to capture forest disturbances occurring at small spatial scales (Diniz et al. 2015). When compared to Landsat data (30 m), for example, MODIS misses majority of forest disturbances (Hansen \& Loveland 2012). To address this problem, near real-time forest change monitoring is now shifting from coarse resolution to medium resolution data (10-30m) (Dutrieux et al. 2015; Reiche, de Bruin, et al. 2015; Hansen et al. 2016). Medium spatial resolution data (e.g. Landsat) have been available long before MODIS data (since 1972), but they are only being used for near real-time forest change monitoring in recent years (Hansen et al. 2016). In the past, medium spatial resolution data were not available for free, thus too expensive to buy (Wulder et al. 2012) especially for large-area forest monitoring, and were not frequent enough (low temporal details) to provide timely information on new forest disturbances, especially in tropical areas (Asner 2001). The availability of multiple Landsat sensors coupled with the advent of the European Union's Copernicus Sentinel satellites, such Sentinel-1 and -2 sensors in recent years, temporally dense free and open access medium resolution data are increasingly becoming available. These multiple data streams provide frequent observations suitable 
for mapping forest disturbances in near real-time. To use these multiple data streams optimally, however, change detection approaches which can combine observations from multiple sensors are needed.

The approaches for detecting forest disturbances from satellite data have also evolved over the years. They shifted from approaches that detect forest disturbances by comparing two satellite images acquired at two different time steps (Coppin et al. 2014) or by using annually aggregated time series of satellite observations (Cohen et al. 2010; Kennedy et al. 2010; Griffiths et al. 2012) to approaches that use all available satellite observations to detect forest disturbances (Reiche, de Bruin, et al. 2015; Verbesselt et al. 2012; Zhu \& Woodcock 2014b; Zhu et al. 2012). The approaches that use the time series of all available satellite observations were developed mainly to address the challenge of seasonality in satellite data, but not inter-sensor differences in multi-sensor time series. Typically, these approaches detect forest disturbance by analysing observations available at a particular pixel, thus relying only on temporal and spectral information. The observations can either be of vegetation index (Verbesselt et al. 2012; Reiche, de Bruin, et al. 2015) or multispectral bands (Hansen et al. 2016; Zhu \& Woodcock 2014b).

Overall, satellite-based forest monitoring has improved significantly over the years, and in principle, a combination of frequent medium resolution data and advanced change detection approaches should allow for accurate and timely detection of forest disturbances. However, generating spatially and temporally accurate forest change alerts is still challenging. Challenges which hinder timely and accurate detection of forest disturbances from satellite image time series are discussed in Sections 1.3.1.

Throughout this thesis, unless stated explicitly, the term "forest disturbance" refers to full or partial loss of forest cover which is visible from high and medium satellite data. It is thus used to encompass both deforestation and forest degradation. The usage of the term "forest change" is also limited to forest cover loss, thus interchangeable with "forest disturbance" unless clearly stated. The term "monitoring" refers to the assessment of the forest cover over time with the aim of detecting forest disturbances.

\subsection{Current challenges facing near real-time satellite-based forest monitoring}

\subsubsection{Seasonality in image time series and persistent cloud cover}

Strong seasonal variations in forest photosynthetic activity and canopy moisture content coupled with persistent cloud cover are the main challenges affecting accurate and timely detection of forest disturbances from satellite image time series. These challenges negatively affect the capacity for change 
detection approaches to identify forest disturbances in satellite image time series. Seasonality in image time series, for example, can disguise lowmagnitude forest disturbances, and can also trigger many false detections. To detect forest disturbances timely and accurately in forests that have strong seasonality, the seasonal component in the image time series should be accounted for. Typically, the seasonal component is accounted for using a seasonal model (Verbesselt et al. 2012; Zhu et al. 2012). The use of a seasonal model is based on the assumption that the seasonal component in the time series can be described mathematically (Cleveland et al. 1990). However, because of persistent cloud cover (Asner 2001) and irregular image acquisition, the satellite image time series are often irregular, thus making it difficult to properly account for seasonality. As a result, near real-time forest change detection in dry tropical forests is difficult.

\subsubsection{Small-scale and low-magnitude forest disturbances}

Detecting small-scale and low-magnitude forest disturbances timely and accurately is still a major challenge for satellite-based forest change monitoring. Oftentimes, forest disturbances which occur at small-scale (Hansen et al. 2016; Tyukavina et al. 2013) or have low-magnitude of change (Cohen et al. 2017) are omitted, even when using 30m resolution Landsat time series. Several factors can contribute to high omission error for smallscale and low-magnitude forest disturbances. First, high variance in the satellite image time series, as a result of seasonality or noise, can disguise the disturbances which have low-magnitude of change, making it difficult to identify such disturbances early. Monitoring forest changes using algorithms which are tuned for high-magnitude disturbances also contributes to the omission of small-scale and low-magnitude forest disturbances (Cohen et al. 2017). In principle, the omission of small-scale and low-magnitude forest disturbances can be reduced by using less strict thresholds when identifying forest disturbances in satellite image time series, but such thresholds can also amplify the commission error (Cohen et al. 2017).

\subsubsection{Inherent noise in satellite image time series}

Timely detection of forest disturbances is often accompanied by high commission error (many false detections) (Reiche, de Bruin, et al. 2015). False detections make near real-time forest change monitoring less reliable. Too many false alerts can increase the cost of policing the forest, because resources might be deployed to many areas where forest disturbances are not truly happening. It can also erode confidence in near real-time forest monitoring systems among the users of the alerts. When seasonality is accounted for, false detections are caused mainly by noise which is inherent 
in the satellite images time series as a result of atmospheric contaminations and sensor artefacts. Climate-induced forest disturbances, which may not lead to forest loss, can also trigger many false detections. For example, drought can trigger abrupt decrease in photosynthetic activity of the forest (Saatchi et al. 2012), and such decrease can be detected during forest change monitoring. Yet, existing forest change detection approaches do not have the capacity to account for the effects of climate extremes.

In recent years, there has been research effort to address the problem of false detections, but the proposed approaches (e.g. Zhu et al. 2012) either reduce temporal accuracy or increase the omission error. Therefore, amplified false detections remain a pressing challenge in satellite-based forest monitoring.

\subsubsection{Inter-sensor differences in multi-sensor time series}

Currently, not a single medium spatial resolution satellite sensor is capable of providing sufficiently frequent observations to support near realtime forest monitoring across the globe. Observations from multiple sensors are needed. However, it is challenging to combine such multi-sensor observations into a temporally consistent time series because of the intersensor differences. Oftentimes, satellite sensors (optical ones) have different radiometric calibrations, viewing angles, orbit times, and spatial and spectral resolutions, and these differences can propagate disjoints in multi-sensor time series (Roy et al. 2016; Zhang \& Roy 2016; Fan \& Liu 2017). Such disjoints can lead to many false abrupt changes in a multi-sensor time series (Tian et al. 2015). Methods for reducing inter-sensor differences in the multi-sensor data have been proposed (Scarino et al. 2016; Roy et al. 2016; Fan \& Liu 2017), but they use one sensor as a reference for other sensors. Using one sensor as a reference is problematic because a new reference sensor is always needed once the initial reference sensor is no longer a good reference. Ideally, methods for reducing inter-sensor differences in multi-sensor time series should be generic and should not rely on one sensor as a reference for others. Inter-sensor differences also exist among the Synthetic Aperture Radar (SAR) sensors. SAR sensors operate at different wavelength, with some operating in C-band (Reiche, Verbesselt, et al. 2015) while others are operating in L-band (Lehmann et al. 2012; Rosenqvist et al. 2007). To overcome the challenge of persistent cloud cover, data from multiple SAR and optical sensors should be integrated to achieve timely detection of forest disturbances (Reiche, de Bruin, et al. 2015). Significant progress has been made towards addressing the challenge of combining SAR and optical time series (Lehmann et al. 2015; Lehmann et al. 2012; Reiche, Verbesselt, et al. 2015) to improve near realtime monitoring of forest disturbances (Reiche et al. 2016; Reiche, de Bruin, et al. 2015), but the proposed approaches for combining SAR and optical time 
series were only tested in areas where forests do not exhibit strong seasonality. As a result, it is not yet known whether the current approaches for combining time series are also applicable to dry forests.

\subsection{Limitations of existing forest change monitoring approaches}

Several forest change monitoring approaches have been proposed over the years (Zhu et al. 2012; Shimabukuro et al. 2006; Verbesselt et al. 2012; Reiche, de Bruin, et al. 2015; Hansen et al. 2016) to improve detection of forest disturbances from satellite data. However, the proposed approaches have major limitations. First, existing forest monitoring approaches only rely on single-pixel-time series to detect forest disturbances from satellite data. They are developed with a view that temporal and spectral information in satellite image time series is sufficient to distinguish forest disturbances from normal forest dynamics. Yet, accounting for seasonality in image time series based on temporal information only is often challenging when the pixel-time series is irregular, contain strong effects of inter-sensor differences or is too short to fit a seasonal model correctly. Second, existing approaches lack capacity to reject false detections without compromising the temporal accuracy or amplifying omission of small-scale and low-magnitude disturbances. Oftentimes, single pixel-time series alone does not contain additional and useful information to immediately differentiate forest disturbances from false detections. Third, existing change detection approaches are not capable of reducing inter-sensor differences in multisensor time series. As a result, the existing change detection approaches are either only applied on a single-sensor time series or on the time series from multiple satellite sensors which have similar characteristics (e.g. Landsat sensors). Those that can integrate time series from different satellite sensors (e.g. Reiche, de Bruin, et al., 2015) are currently challenging to apply in dry tropical forests because of strong seasonality.

\subsection{Integrating contextual information in satellite-based forest change monitoring}

Forest disturbances and natural forest dynamics are spatio-temporal events and processes. They have both spatial and temporal contexts, thus autcorrelated in space and time. Yet, existing change detection approaches for forest change monitoring do not include contextual information when detecting forest disturbances from satellite data. Contextual information in this thesis refers to information derived from neighbouring pixels of a focal pixel - a pixel that is being analysed for forest disturbance. I used the terms contextual information and spatial information interchangeably in this thesis. 
Forest change monitoring can potentially be improved by including contextual information during forest change detection. For example, contextual information has potential to reduce seasonal variations in satellite data through spatial normalisation. This is because undisturbed pixels which are closer to each other are likely to exhibit similar temporal dynamics. With spatial normalisation, unlike with seasonal model, seasonality problem can be addressed even when the image time series is irregular or temporally short. We can potentially use spatial normalisation to also tackle the problem of inter-sensor differences in multi-sensor image time series. If each image is normalised individually using contextual information, the differences in multi-sensor time series caused for example by differences in radiometric calibrations may reduce. Using contextual information also has potential to reduce false detections. Since forest disturbances are spatio-temporal events, we can for example combine contextual and temporal information in satellite data to detect anomalies at pixel level, and subsequently use a set of spatiotemporal metrics extracted from satellite data cubes to confirm forest disturbances. If a set of spatio-temporal metrics can help us to discriminate forest disturbances from false detections, then we can also probably be able to tackle the problem associated which high omission error for small-scale and low-magnitude disturbances. This is because, instead of using a strict threshold to identify forest disturbances, we can potentially use less strict thresholds to detect such disturbances, and use the spatio-temporal metrics to reject false detections.

\subsection{Research objectives}

The overall objective of this thesis is to improve satellite-based forest change monitoring by addressing the key challenges which hinders accurate and timely detection of forest disturbances from satellite data. More specifically, the thesis assesses whether the problem of seasonality, omission of small-scale and low-magnitude forest disturbances, and inherent noise in satellite image time series and inter-sensor differences in multi-sensor image time series can be addressed by combining contextual and temporal information when monitoring forest disturbances using satellite data. The research presented in this thesis is guided by the following research questions:

1. How do we account for seasonality in irregular satellite image time series when aiming for accurate and timely detection of disturbances in dry forests? 
2. What is the added-value of using both spatial and temporal information when monitoring forest disturbances from satellite image time series?

3. How can we combine observations from multiple satellites using space-time information to improve near real-time forest change monitoring?

\subsection{The structure of this thesis}

This thesis consists of seven chapters, including this introductory chapter. Figure 1.1 shows the linkage between the chapters, with arrows indicating how the chapters build up to each other.

Chapter 2 addresses the first research question (RQ1) by proposing a spatial normalisation approach for reducing seasonality in a combined optical time series for Landsat-5/TM and Landsat-7/ETM+. The proposed approach is compared with a seasonal model approach, and was tested at two study sites. One study site is dry forest area, located in south east of Santa Cruz de la Sierra, Bolivia, where forest is characterised by strong seasonality in its photosynthetic activity. Forest disturbances at this site are dominated mainly by industrial agricultural expansion with large blocks of deforestation events. The other site is an evergreen forest area, located in west of Ariquemes, Rondonia State, Brazil. At this site, forest disturbances are heterogeneous and correspond mostly to a process of colonisation. Forest disturbances were detected using existing change detection method that analyses single pixeltime series to detect forest disturbances (Verbesselt et al. 2012).

Chapter 3 tackles the second research question (RQ2) by developing a space-time change detection approach that detects forest disturbances by exploiting both spatial and temporal information in satellite data cubes. As in Chapter 2, this approach is evaluated at a dry tropical forest site in Bolivia and a humid tropical forest site in Brazil using a combined time series from Landsat-5/TM and Landsat-7 /ETM+. The spatial normalisation approach proposed in Chapter 2 was used to reduce seasonality in the image time series.

Chapter 4 also addresses the second research question (RQ2) by identifying the "space-time features" which are important for accurate and timely detection of forest disturbances. This chapter uses the space-time features derived from satellite data cubes to increase the capacity for rejecting false detections without compromising temporal accuracy. This chapter builds upon Chapter 2 and Chapter 3. The idea of space-time features approach was evaluated in the UNESCO Kafa Biosphere Reserve, situated in the southwest of Ethiopia, where small-scale and gradual disturbance processes, caused mainly by small-holder agriculture, human settlements 
expansion, industrial coffee plantations, and domestic firewood and charcoal extractions are common(Pratihast et al. 2014; Dresen et al. 2014; Devries, Verbesselt, et al. 2015). Forest disturbances were detected using a combined time series of Landsat-7/ETM+ and Landsat-8/OLI.

Chapter 5 combines time series from Landsat and Sentinel-2 sensors to address the third research question (RQ3). In particular, this chapter assessed whether the spatial context approach proposed in Chapter 2 can also be used to reduce inter-sensor differences in multi-sensor time series of optical sensors. It also evaluated how detection of small-scale disturbances improves with increasing spatial resolution $(10 \mathrm{~m})$, and whether increasing temporal density of medium spatial resolution observations also improves the estimates for forest loss. In this chapter, forest disturbances are detected using the space-time approaches developed in Chapter 3 and 4. The approach presented in this chapter is demonstrated in the UNESCO Kafa Biosphere Reserve, situated in the southwest of Ethiopia.

Chapter 6 tackles RQ1 and RQ3. First, it evaluates whether the spatial normalisation approach developed in Chapter 2 can also be used to reduce seasonal variations in SAR time series to facilitate SAR-Optical multi-sensor data integration to detect forest disturbances in near real-time. Second, it evaluates how the timeliness for forest change detection improves when combining observations from multiple sensors. A probabilistic change approach that combines SAR-optical time series (Reiche, de Bruin, et al. 2015) was used to detect forest disturbances after reducing seasonality using spatial context. The probabilistic change detection approach converts the original SAR and optical information into non-forest probabilities, thus removing inter-sensor differences in the multi-sensor time series.

Chapter 7 presents the main findings of this thesis. The findings are discussed in the context of the research questions, and the remaining research gaps are highlighted. In particular, the prospects for space-time forest change detection using SAR-optical image time series to take advantage of the space-time features is discussed. 


\section{Single pixel-time series change analysis}

Space-time change analysis

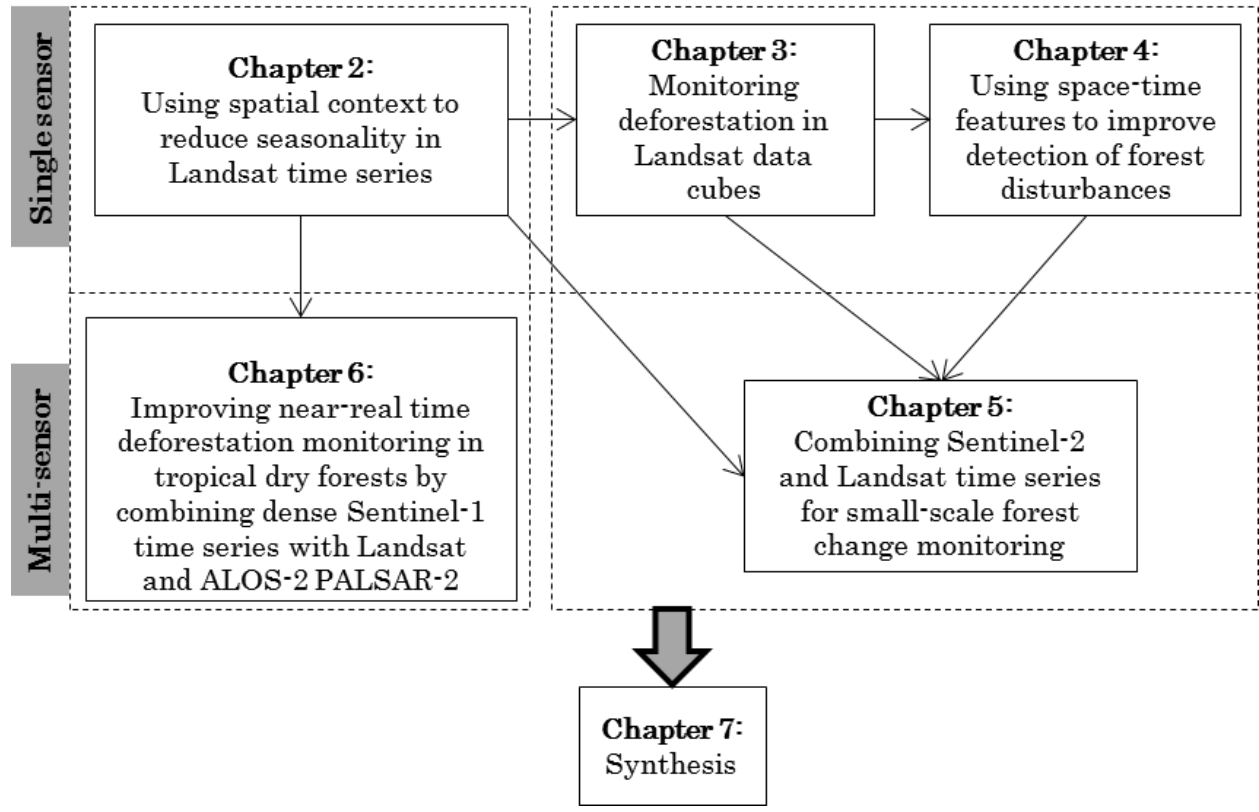

Figure 1.1: Overview of the chapters (excluding Chapter 1) for this thesis 



\section{Using spatial context to improve early detection of deforestation from Landsat time series}

This chapter is based on:

Hamunyela, E., Verbesselt, J., \& Herold, M. (2016). Using spatial context to improve early detection of deforestation from Landsat time series. Remote Sensing of Environment, 172, 126-138. 


\section{Abstract}

Mapping deforestation using medium spatial resolution satellite data (e.g. Landsat) is increasingly shifting from decadal and annual scales to subannual scales in recent years, but this shift has brought new challenges on how to account for seasonality in the satellite data when detecting deforestation. A seasonal model is typically used to account for seasonality, but fitting a seasonal model is difficult when there are not enough data in the time series. Here, we propose a new approach that reduces seasonality in satellite image time series using spatial context. With this spatial context approach, each pixel value in the image is spatially normalised using the median value calculated from neighbouring pixels whose pixel values are above $90^{\text {th }}$ percentile. Using Landsat data, we compared our spatial context approach to a seasonal model approach at a humid tropical forest in Brazil and a dry tropical forest with strong seasonality in Bolivia. After reducing seasonal variations in Landsat data, we detected deforestation from the same data using Breaks For Additive Season and Trend (BFAST) method. We show that, in dry tropical forest, deforestation events are detected much earlier when the spatial context approach is used to reduce seasonal variations in Landsat data than when a seasonal model is used. In the dry tropical forest, the median temporal detection delay for deforestation from the spatial context approach was two observations, seven times shorter than the median temporal detection delay from the seasonal model approach (15 observations). In the humid tropical forest, the difference in the temporal detection delay between spatial context and seasonal model approach was not significant. The differences in overall spatial accuracy between the spatial context and seasonal model were also not significant in both dry and humid tropical forests. The main benefit for using spatial context is early detection of deforestation events in forests with strong seasonality. Therefore, the spatial context approach we propose here provides opportunity to monitor deforestation events in dry tropical forests at sub-annual scales using Landsat data.

Keywords: Landsat, deforestation, spatial context, seasonality, seasonal model 


\section{$2.1 \quad$ Introduction}

Mapping deforestation using medium spatial resolution satellite data (e.g. Landsat) is increasingly shifting from decadal (Achard et al. 2014) and annual scales (Devries, Verbesselt, et al. 2015; Souza, Jr et al. 2013; Griffiths et al. 2012; Kennedy et al. 2010) to sub-annual scales (Reiche, Verbesselt, et al. 2015; Reiche, de Bruin, et al. 2015; Dutrieux et al. 2015) mainly because of increased temporal availability of medium spatial resolution satellite data in recent years. Mapping deforestation from medium spatial resolution satellite data at sub-annual scales is beneficial because it provides opportunity for timely detection of small deforestation events that cannot be detected from coarse spatial resolution data. Currently, however, detecting deforestation from medium spatial resolution satellite data at sub-annual scales is challenging especially in forests that exhibit strong seasonality in their photosynthetic activity. Satellite images from medium spatial resolution satellite sensors are often not acquired regularly in all parts of the globe. Methods (e.g. DeVries et al., 2015; Verbesselt, Zeileis, \& Herold, 2012; Zhu \& Woodcock, 2014), which are used to detect deforestation at sub-annual scales from satellite image time series, account for seasonality in the time series using a seasonal model. The use of a seasonal model is based on an assumption that there is an identifiable seasonal pattern in the time series which can be described mathematically (Cleveland et al. 1990). However, this assumption may not always hold if the time series is for satellite images which are not acquired at regular interval, or have wide temporal gaps due to persistent cloud cover (Asner 2001). Oftentimes, however, a seasonal model is still used to account for seasonality in such image time series (Zhu \& Woodcock 2014b; Devries, Verbesselt, et al. 2015). Yet, we do not know if using a seasonal model to account for seasonality in satellite image time series which is not acquired at regular interval affects our ability to detect deforestation events early.

Recently, time series from coarse resolution sensors (e.g. Moderate Resolution Imaging Spectroradiometer) have been used to derive the seasonal patterns when mapping deforestation at sub-annual scales from medium spatial resolution satellite data (Dutrieux et al. 2015).The approach of using time series from coarse resolution sensors to address the problem of seasonality in medium spatial resolution satellite data is novel and can be viewed as a synergistic way of using satellite data (De Sy et al. 2012; Zhang 2010; Reiche, Verbesselt, et al. 2015). The weakness of this approach, however, is its reliance on data from another sensor to account for seasonality in Landsat data. If the sensor producing data that are used to derive the seasonal pattern fails, the approach would also not work anymore. To avoid such situation, methods that can address the issue of seasonality in image 
time series without using data from other satellite sensors to derive the seasonal pattern are critically needed and should be developed.

Instead of using a seasonal model to account for seasonality in image time series, we can potentially use spatial context. Here, spatial context refers to spatial neighbourhood of a focal pixel - the pixel which is being processed. There are several ways we can use spatial context to address the challenge of seasonality in image time series. For example, we can assume that pixels within a spatial neighbourhood of a focal pixel are likely to exhibit temporal dynamics similar to that of the focal pixel. Based on this assumption, we can simply calculate similarity between values of the neighbouring pixels and the focal pixel at each time step, resulting into a time series of similarity measures (Lhermitte et al. 2011). Similarity measures calculated during the growing season are likely to be similar to those calculated during senescence. In this way, deforestation can then be mapped from the time series of similarity measures. To calculate similarity measures in a consistent manner, however, reference pixels need to be selected and such pixels themselves should remain stable for the period under consideration. The challenge, however, is on how to keep the same cohort of reference pixels throughout the entire time series because, at some time steps, selected reference pixels might be affected by clouds, and would be masked out by cloud masking algorithm. Such challenge makes the method of calculating similarity measures to minimise the seasonal variations in image time series cumbersome. Another spatial context method that can be used to tackle the problem of seasonal variations in image time series is the one applied in vegetation regeneration studies. In such studies, regeneration of vegetation after fire is usually characterised from satellite data by calculating the ratio between burned plot pixels and un-burned plot pixels at each time step, resulting into the so-called regeneration index time series (Riano et al. 2002; Lhermitte et al. 2010). In a simplistic view, such method can be viewed as local normalisation of pixel values using spatial context. The problem with such method, however, is that pixels which are similar to the focal pixel prior to a fire event should be used as a reference when calculating regeneration index (Lhermitte et al. 2010). Such requirement has some crucial advantages, but is equally problematic because if the reference pixels happen to be disturbed at some subsequent time steps they can no longer be useful references. A new method that does not require selection of reference pixels when using spatial context to tackle the problem of seasonal variations in image time series is, therefore, desirable.

In this chapter, our objectives were i) to investigate how spatial context can be used to reduce seasonal variations in satellite image time series, and ii) to assess whether using spatial context to reduce seasonal variations in satellite image time series leads to earlier detection of deforestation events than when a seasonal model is used. To achieve these objectives, we proposed 
a new spatial context approach for reducing seasonal variations in satellite image time series, and compared the results from the spatial context approach to those where a seasonal model is used to account for seasonality. Normalised difference vegetation index (NDVI) (Tucker 1979) derived from Landsat Thematic Mapper (TM 5) and Enhanced Thematic Mapper Plus (ETM+ 7) images spanning from April 1984 to December 2014 was used as an indicator of vegetation temporal dynamic. Deforestation events were detected using Breaks For Additive Season and Trend (BFAST, Verbesselt et al., 2012), a change detection method capable of detecting forest cover disturbances at sub-annual scales (Dutrieux et al., 2015; Reiche, Verbesselt, et al., 2015). BFAST was first proposed by Verbesselt, Hyndman, Newnham, \& Culvenor (2010) and later optimised by Verbesselt et al. (2012) for near real-time detection of disturbances from satellite image time series based on structural change monitoring framework (Chu et al. 1996; Zeileis et al. 2005; Leisch et al. 2000). With BFAST, the history and monitoring periods are first defined. History period contains the historical data whereas the monitoring period contains newly acquired observation that should be assessed for disturbance. To assess the newly acquired observation for disturbance, regression coefficients are first estimated from the historical data, and subsequently used to predict the value of an incoming observation in the monitoring period. Approaches for testing for structural change in the time series (Chu et al. 1996; Zeileis et al. 2005) are then used to assess if the incoming observations are significantly different when compared to the prediction based on the history period. We hypothesised that deforestation events would be detected much earlier from the time series whose seasonality is reduced using spatial context than from time series whose seasonality is accounted for using a seasonal model.

\section{$2.2 \quad$ Study area}

Our study focused at two tropical forest sites, a humid and dry forest (Figure 2.1). The humid forest site, covering an area of about $10000 \mathrm{~km}^{2}$, is located west of Ariquemes, Rondonia State, Brazil (centred at: $10.2952^{\circ} \mathrm{S}$, $64.0478^{\circ} \mathrm{W}$ ). The dry forest site, also covering $10000 \mathrm{~km}^{2}$, is located in south east of Santa Cruz de la Sierra, Bolivia (centred at: $18.388^{\circ} \mathrm{S}, 62.361^{\circ} \mathrm{W}$ ). These two sites have similar rainfall pattern throughout the year, but the Brazilian site receive higher rainfall per month (Figure 2.2). Compared to the Bolivian site, the study site in Brazil is closer to the equator, and this may explain why the rainfall per month is much higher. This high rainfall per month also means more cloud cover and atmospheric contamination in the satellite data. The forest at the Brazilian site exhibits weak seasonality in its photosynthetic activity, typical of an evergreen tropical forest (Figure 2.3). In contrast, the forest at the Bolivian site is characterised by strong seasonality 
in its photosynthetic activity, typical of dry tropical forest (Figure 2.3). Deforestation at the Bolivian site is dominated mainly by industrial agricultural expansion with large blocks of deforestation events whereas at the Brazilian site deforestation events are heterogeneous and correspond mostly to a process of colonization. The forests at these two sites have varying degree of seasonality, thus providing an ideal opportunity to evaluate whether using spatial context to reduce seasonal variations in image time series is more beneficial than using a seasonal model when detecting deforestation at sub-annual scales.

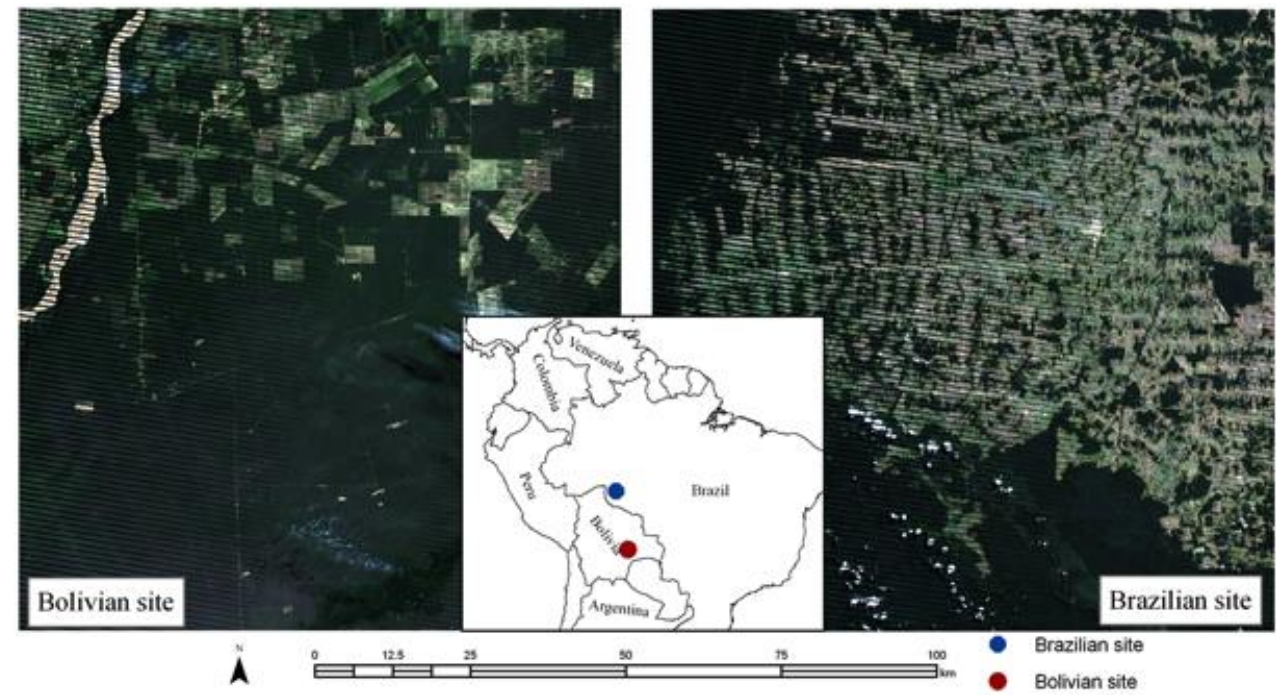

Figure 2.1: Overview of study sites. One study site, a humid forest, is located west of Ariquemes, Rondonia State, Brazil, and the other site, a dry forest, is located south east of Santa Cruz de la Sierra, Bolivia. The base images are band 1-2-3 composites of Landsat ETM+ images from 2013-04-15 (Bolivian site) and 2014-07-21 (Brazilian site). 


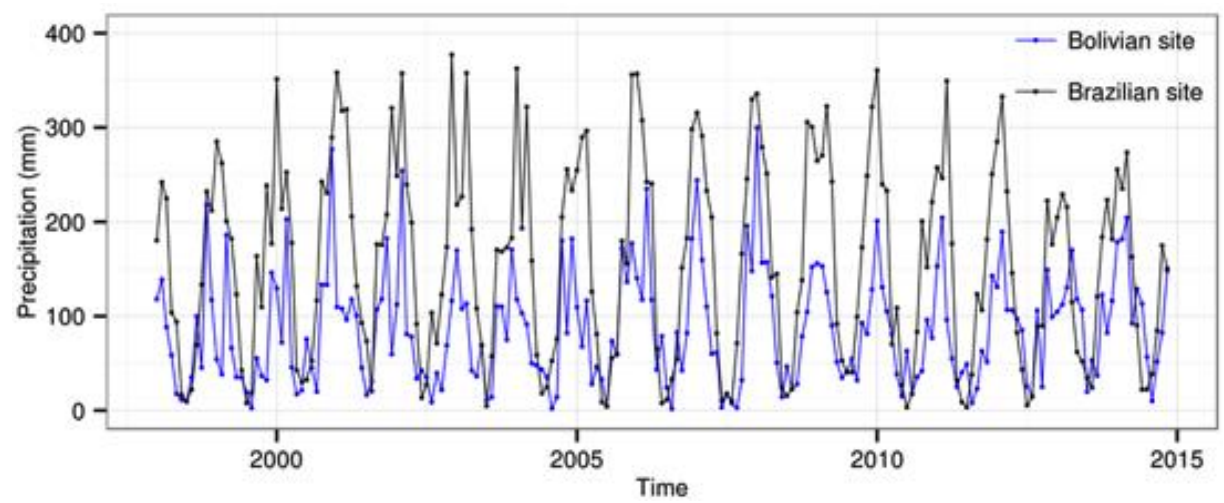

Figure 2.2: Monthly precipitation (mm) for the period 1998 -2014 measured by the tropical rainfall measuring mission (TRMM) for the Bolivian and Brazilian study site. The time series are spatially-averaged, and were obtained through the Giovanni online data system (http://disc.sci.gsfc.nasa.gov/giovanni).

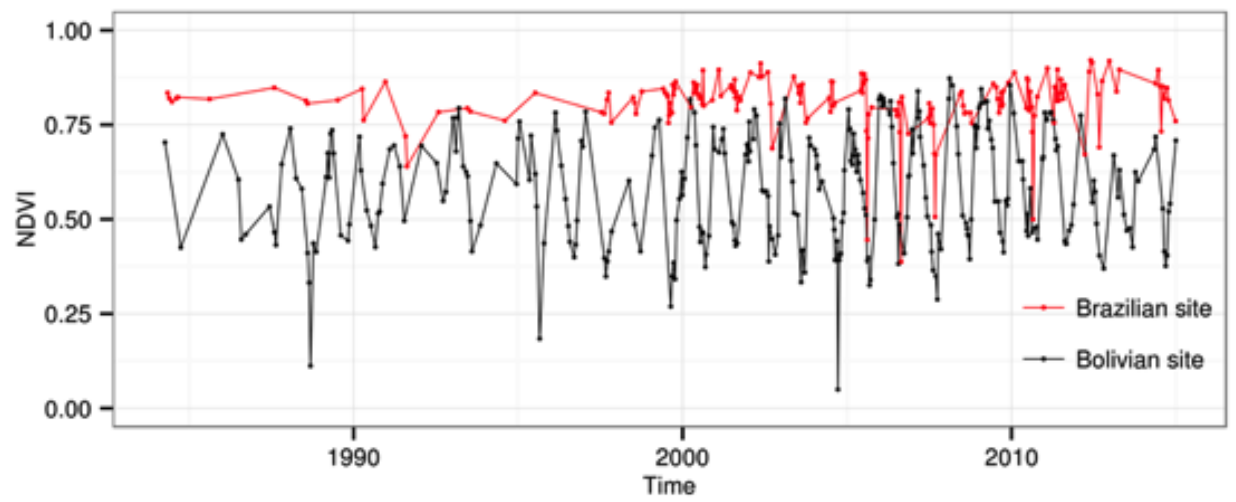

Figure 2.3: Time series for Normalised difference vegetation index (NDVI) for period of 1984 - 2014 derived from Landsat Thematic Mapper (TM 5) and Enhanced Thematic Mapper Plus (ETM+ 7) at the Brazilian and Bolivian study sites.

\subsection{Data and pre-processing steps}

\subsubsection{Landsat data and tree cover percent dataset}

We used NDVI time series derived from terrain corrected (L1T) Landsat TM 5 and ETM+7 images, spanning from April 1984 to December 2014. Landsat images were obtained from The United State of America's Geological Survey (USGS) Landsat Surface Reflectance (SR) Climate Data Records 
(CDR). Landsat SR CDR Products are atmospherically and geometrically corrected. In total, $448 \mathrm{~L} 1 \mathrm{~T}$ images were used at the Bolivian study site, and $228 \mathrm{~L} 1 \mathrm{~T}$ images at the Brazilian study site. Clouds and cloud-shadows were masked out using the Function of mask (Fmask) outputs (Zhu \& Woodcock 2012), which were distributed with Landsat SR CDR Products by The United State of America's Geological Survey (USGS). We masked non-forest areas using the Landsat tree cover continuous fields for 2005 (Sexton et al. 2013).To ensure that we do not analyse pixels with insufficient data, we also masked pixels which had less than 15 observations in their entire time series and less than 6 observations in the historical period - the period prior to year 2005.

\subsubsection{Validation data}

A total of 468 sample points were used to validate the final change map at the Bolivian site, whereas 400 sample points were used at the Brazilian site (Table 2.1). Another 82 sample points were used as training data set at the Bolivian site, whereas 70 sample points were used at the Brazilian site (Table 2.1). Validation data were collected from Landsat images, complemented by high spatial resolution imagery available in Google Earth (https://www.google.com/earth/) and Bing Maps (http://www.bing.com/maps/), through visual interpretation. We followed a proportional probability sampling when collecting the validation data. The maps for deforested and stable forest areas were used as strata (see Section 2.4.7).

Table 2.1: The number of sample points which were used for training purposes and final validation of deforestation maps produced at the Bolivian and Brazilian site. Training dataset was used to calibrate Breaks For Additive Season and Trend (BFAST) for deforestation detection, whereas the test dataset was used to validate the deforestation map produced after calibrating BFAST.

\begin{tabular}{lcccc}
\hline & \multicolumn{2}{c}{ Bolivian site } & \multicolumn{2}{c}{ Brazilian site } \\
\hline & Forest & Deforested & Forest & Deforested \\
\hline Training data & 74 & 8 & 49 & 21 \\
Test data & 421 & 47 & 280 & 120 \\
\hline
\end{tabular}

\section{$2.4 \quad$ Methods}

First, we proposed a new spatial context approach for reducing seasonal variations in image time series, and applied it to Landsat NDVI time series, resulting in a new time series that we referred to as spatially normalised NDVI time series (Section 2.4.1). Second, we tested eight varying spatial window sizes at each study site to determine the optimal window size to calculate spatially normalised NDVI, and determined the computational time 
per pixel for each window (Section 2.4.2). Third, we removed the negative outliers from Landsat NDVI and spatially normalised NDVI time series (Section 2.4.3). Fourth, we optimised BFAST algorithm for deforestation detection using the magnitude of change (Section 2.4.4). Fifth, deforestation was detected from spatially NDVI (Section 2.4.5) and original NDVI (Section 2.4.6) time series using BFAST. Finally, we explain how validation data were collected (Section 2.4.7) and how the final deforestation maps were validated (Section 2.4.8).

\subsubsection{Using spatial context to reduce seasonal variations in Landsat NDVI time series}

Vegetation indices derived from satellite data are used as proxy indicators of the state and condition of the forest. A major negative deviation in the time series of a vegetation index, not related to seasonality, can therefore be used to signal forest disturbance, for example deforestation. Seasonal variations, however, can be mistaken for deforestation if not properly removed from the time series. So far, seasonal model is the only approach used to account for seasonality in satellite image time series. Yet, fitting a seasonal model is difficult when there is not enough data in the time series. Here, we propose an alternative method for reducing seasonal variations in image time series using spatial context. To reduce seasonal variations using spatial context, we propose that i) a median value should be computed from the pixels within a defined spatial neighbourhood, which are above a certain percentile (e.g. 90th), and ii) divide the value of the focal pixel using the calculated median value. When this procedure is applied at each time step, it would result into a spatially normalised time series from which deforestation can be detected using existing generic change detection methods (e.g. Verbesselt et al., 2012; Zhu \& Woodcock, 2014). We base our approach on the assumption that, if no disturbance has occurred, the forest at the focal pixel would exhibit temporal dynamics similar to that of forest pixels within its neighbourhood. Similarly, we exploit the fact that climatic influences (e.g. drought) occur at more regional scales and deforestation events at more local scales. As such, climate influences can easily be removed from the time series when dividing the value of the focal pixel with a median value computed from the pixels within a defined spatial neighbourhood. Dividing the value of the focal pixel with median value of its neighbours would yield a value closer to one if no disturbance has occurred or if the disturbance is regional. When the focal pixel becomes disturbed and the disturbance is local, dividing the value of the focal pixel by the median value calculated from pixels in the neighbourhood, which are above a certain percentile, is likely to amplify the impact of the disturbance on the time series. 
To spatially normalise each image, we implemented a spatial moving window whereby a $90^{\text {th }}$ percentile is calculated from pixel values which are within the spatial window, and subsequently calculate the median value (VImedian) from the value of the pixels that are above or equal to the $90^{\text {th }}$ percentile. In a next step, the value of the focal pixel ( $\left.\mathrm{VI}_{\text {pixel }}\right)$ is divided by the $\mathrm{VI}_{\text {median }}$ (Eq. 2.1), resulting in what we refer to as spatially normalised NDVI (sNDVI).

$$
\text { sNDVI }=\frac{V I_{\text {pixel }}}{V I_{\text {median }}}
$$

When the spatial normalisation procedure is applied to all pixels in the image, and applied at each time step we obtain sNDVI time series (Figure 2.4). Deforestation can then be detected from sNDVI time series using time series-based change detection methods (e.g. Verbesselt et al. 2012). It is important that the $\mathrm{VI}_{\text {pixel }}$ is normalised with a value calculated from forest pixels, to avoid normalising with a value from deforested area. Therefore, in

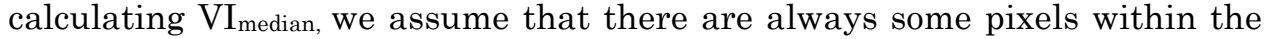
moving window which are not disturbed, and these pixels would be either above or equal to the $90^{\text {th }}$ percentile of all pixels in the moving window. If we calculate $\mathrm{VI}_{\text {median }}$ from disturbed pixels, and $\mathrm{VI}_{\text {pixel }}$ is also disturbed, the impact of the disturbance may be smoothed out from the time series when dividing $\mathrm{VI}_{\text {pixel }}$ by $\mathrm{VI}_{\text {median. }}$ So, by calculating $\mathrm{VI}_{\text {median }}$ from pixels that are above or equal to the 90th percentile, we minimise the chance of calculating $\mathrm{VI}_{\text {median }}$ from disturbed pixels. Although we used $90^{\text {th }}$ percentile here, other percentiles (e.g. $95^{\text {th }}$ ) can also be used when calculating $\mathrm{VI}_{\text {median }}$. 


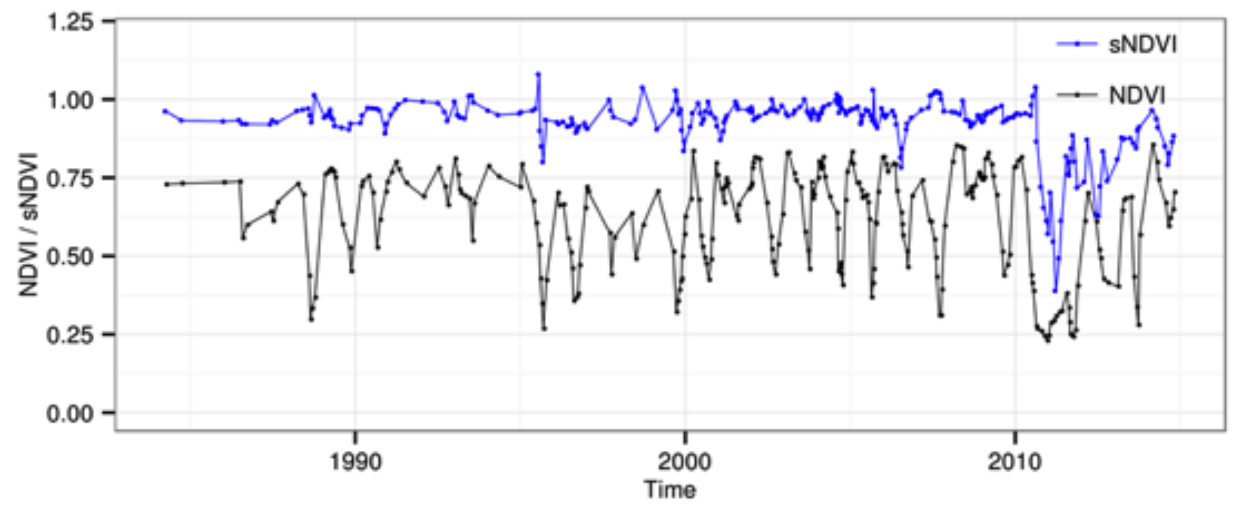

Figure 2.4: An example of normalised difference vegetation index (NDVI) and spatially normalised difference vegetation index (sNDVI) time series for period of 1984 - 2014 calculated from Landsat Thematic Mapper (TM 5) and Enhanced Thematic Mapper Plus (ETM+ 7) images at the Bolivia study site. The NDVI and sNDVI are from the same Landsat pixel.

\subsubsection{Window size}

The window size is an important aspect for our spatial context method. The spatial moving window needs to be sufficiently larger than the size of the deforestation event to avoid smoothing out the disturbance impact from the time series when dividing $\mathrm{VI}_{\text {pixel }}$ by $\mathrm{VI}_{\text {median, }}$ and also for it to capture local variations. We evaluated how the window size influence the detection of deforestation from sNDVI time series, by testing eight window sizes, ranging from 7.29 ha to 1317.69 ha (Table 2.2). We also assessed how the size of spatial window influences computational time, the time it takes to complete processing a single pixel when calculating sNDVI. An optimal window size should yield high mapping accuracy and relatively low computational cost.

Table 2.2: The size of spatial windows used to calculate spatially normalised vegetation index (sNDVI) from Landsat normalised deference vegetation index (NDVI).

\begin{tabular}{lccc}
\hline $\begin{array}{l}\text { Window } \\
\text { name }\end{array}$ & $\begin{array}{c}\text { Window dimension } \\
\text { (row x column) }\end{array}$ & Number of Landsat pixels & $\begin{array}{c}\text { Window } \\
\text { size (ha) }\end{array}$ \\
\hline W9 & $9 * 9$ & 81 & 7.29 \\
W25 & $25 * 25$ & 625 & 56.25 \\
W35 & $35 * 35$ & 1225 & 110.25 \\
W41 & $41 * 41$ & 1681 & 151.29 \\
W61 & $61 * 61$ & 3721 & 334.89 \\
W81 & $81 * 81$ & 6561 & 590.49 \\
W101 & $101 * 101$ & 10201 & 918.09 \\
W121 & $121 * 121$ & 14641 & 1317.69 \\
\hline
\end{tabular}




\subsubsection{Removing noise from NDVI and sNDVI time series}

Satellite-based products (e.g. NDVI) usually contain noise (Vermote et al. 2009). So, our time series of Landsat NDVI, and by extension the sNDVI, were also noisy, characterized by negative outliers, which possibly are a result of factors such as cloud contamination and atmospheric scatter that were not captured by Fmask algorithm. Such negative outliers can be detected as if they are deforestation thus resulting in high commission error. To remedy this problem, we removed the negative outliers from original NDVI and sNDVI time series using the method proposed by Hamunyela et al. (2013) (Eq.2.2).

$$
x_{t}=\frac{x_{t-1}+x_{t+1}}{2}\left\{\text { if } x_{t}-x_{t-1}<-0.01 x_{t-1} \& x_{t}-x_{t+1}<-0.01 x_{t+1}\right\}
$$

where $x_{t}$ is an observation in the time series at time $t, x_{t-1}$ is the observation in the time series at time $t-1$, and $x_{t+1}$ is the observation at time $t+1$. Observation $x_{t}$ is replaced as an outlier with the average of $x_{t-1}$ and $x_{t+1}$ if the difference between $x_{t}$ and $x_{t-1}$ is less than -1\% of $x_{t-1}$, and the difference between $x_{t}$ and $x_{t+1}$ is also less than $-1 \%$ of $x_{t+1}$. This method is however not able to remove outliers which are consecutive. Figure 2.5 show an example of how local outliers were removed the sNDVI time series.

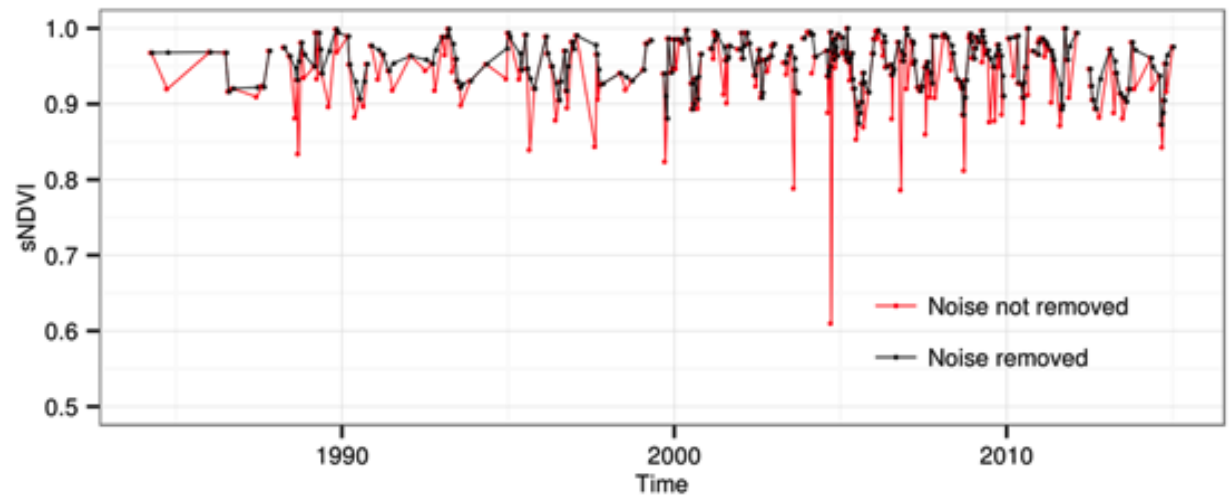

Figure 2.5: An example of how local outliers are removed from the time series using the method proposed by Hamunyela et al. (2013).

\subsubsection{Optimising BFAST for deforestation detection}

BFAST is a generic change detection method (Verbesselt et al. 2012), and it is not calibrated to differentiate deforestation from other disturbances. Therefore, when a negative breakpoint is detected in the image time series using BFAST, it does not necessarily imply deforestation. Magnitude of 
change can be used to optimise BFAST to better differentiate deforestation from other disturbances (Devries, Verbesselt, et al. 2015). To map deforestation from Landsat NDVI and sNDVI time series therefore, we determined the optimal change magnitude that can be used as threshold for accepting the change detected by BFAST as deforestation. To determine the optimal change magnitude $\left(m c_{s}\right)$, we created a sequence of magnitudes $(\mathrm{n}=$ 500) ranging from -0.5 to 0 , at an interval of -0.001 . Each of these magnitudes was then used as a magnitude threshold $\left(m c_{t}\right)$ for deforestation while applying BFAST to the time series of each pixel which was selected for training purposes. Sequentially, each observation in the monitoring period was evaluated for disturbance, and deforestation was declared if the calculated magnitude of change $\left(m c_{\rho}\right)$ is higher than the pre-defined magnitude threshold $\left(m c_{t}\right)$.We then used the training data to calculate the overall spatial accuracy achieved when using each of the magnitude threshold as a threshold for deforestation. The magnitude threshold that achieved the highest overall spatial accuracy (OSA) was chosen as the optimal magnitude threshold (Table 2.2). We determined the optimal magnitude threshold ( $m c_{s}$ ) for NDVI (seasonal model approach) and sNDVI (seasonal model approach) separately at each study site.

Table 2.3: Optimal magnitude thresholds $\left(\mathbf{m c}_{\mathbf{s}}\right)$ which were used to calibrate BFAST when mapping deforestation from normalised vegetation index (NDVI) and spatially normalised difference vegetation index (sNDVI) time series at the Bolivian and Brazilian study site. The optimal magnitude thresholds were derived by calculating the overall spatial accuracy (OSA) using the training data set. Magnitude thresholds that achieved the highest OSA were the optimal magnitude threshold.

\begin{tabular}{lcccc}
\hline & \multicolumn{2}{c}{ Bolivian site } & \multicolumn{2}{c}{ Brazilian site } \\
\cline { 2 - 5 } & OSA (\%) & $m c_{s}$ & OSA (\%) & $m c_{s}$ \\
\hline NDVI & 95.1 & -0.185 & 85.7 & -0.202 \\
sNDVI from W9 & 92.7 & -0.132 & 85.7 & -0.072 \\
sNDVI from W25 & 93.9 & -0.206 & 91.4 & -0.115 \\
sNDVI from W35 & 93.9 & -0.203 & 88.6 & -0.108 \\
sNDVI from W41 & 93.9 & -0.383 & 88.6 & -0.113 \\
sNDVI from W61 & 93.9 & -0.210 & 87.1 & -0.090 \\
sNDVI from W81 & 93.9 & -0.377 & 85.7 & -0.093 \\
sNDVI from W101 & 93.9 & -0.198 & 88.6 & -0.098 \\
sNDVI from W121 & 92.7 & -0.132 & 87.1 & -0.100 \\
\hline
\end{tabular}

\subsubsection{Detecting deforestation from sNDVI time series}

We detected deforestation from sNDVI time series using BFAST (Verbesselt et al. 2012). We focused on detecting deforestation events that occurred in 2005 through 2014 at each study site. The detection of 
deforestation was done in a sequential manner whereby each observation in the monitoring period $(2005-2014)$ was treated as an incoming observation. We mimicked a monitoring scenario whereby an incoming observation is immediately assessed for deforestation. BFAST, as described by Verbesselt et al. (2012) detects breakpoints in a 'near real-time mode' within the time series by first predicting the value for the incoming observation $\left(y_{t}\right)$ using a regression equation Eq.2.3 if the trend and seasonal terms are not included.

$$
\hat{y}_{t}=\beta_{0}+\varepsilon
$$

whereby $\hat{y}_{t}$ is the predicted value of $y_{t}, \quad \beta_{0}$ is the intercept estimated by regressing previous observations (historical data) on time (acquisition date of the image); and $\varepsilon$ is the random error. The estimation of $\beta_{0}$ is done using the ordinary least squares (OLS). Next, residuals are computed by subtracting $y_{t}$ from $\hat{y}_{t}$. Moving sum (MOSUM) of residuals is then calculated using Eq. 2.4 :

$$
M O_{t}=\frac{1}{\sigma \sqrt{n}} \sum_{s=t-h+1}^{t}\left(y_{s}-\hat{y}_{s}\right)
$$

where $h$ is the size of the moving window calculated as a fraction of the number of observations $(n)$ in the historical period, $t$ is time, and $\sigma$ is an estimator of the variance (Leisch et al. 2000; Zeileis et al. 2005). Throughout this study, we assigned $25 \%$ of $n$ as the size (h) for the moving window when calculating the MOSUM of residuals. In a subsequent step, we test if the residuals have crossed the boundary of its empirical fluctuation whereby the boundary is the confidence interval (e.g. 95\%) specified by the user. Empirical fluctuation of MOSUM residuals is basically the historical oscillation of MOSUM of residuals estimated from historical data. The MOSUM of residuals $\left(M O_{t}\right)$ is expected to remain within the boundary of its empirical fluctuation (Leisch et al. 2000) if $\beta_{0}$ is a good predictor of the incoming observation (Chu et al. 1996; Zeileis et al. 2005). A 5\% significant level was used as threshold to either accept or reject the null hypothesis that the MOSUM of residuals has not crossed the boundary of its empirical fluctuation. The assumption is that if the photosynthetic activities of the forest have not been disturbed, the incoming observation $\left(y_{t}\right)$ can be estimated reliably by $\beta_{0}$. A breakpoint is signalled when the $M O_{t}$ crosses the boundary of its empirical fluctuation (Zeileis et al. 2010; Zeileis et al. 2005; Verbesselt et al. 2012). When a breakpoint is detected in the time series based on $M O_{t}$, we calculate the magnitude of change $\left(m c_{\rho}\right)$ by subtracting the predicted value $\left(\hat{y}_{t}\right)$ from incoming observation $\left(y_{t}\right)$ where a breakpoint is detected (Eq. 2. 5). Next, we compare $m c_{\rho}$ and $m c_{s}$, and deforestation is signalled if $m c_{\rho}$ is negative, and $\left|m c_{\rho}\right|$ is greater than $\left|m c_{s}\right|$. If deforestation is 
signalled, the monitoring of deforestation at that particular pixel is ceased, otherwise, the monitoring at such pixel continues until each of subsequent observation is evaluated. With this monitoring approach, if an area had experienced multiple deforestation events between 2005 and 2014, only one of the events would be detected.

$$
m c_{\rho}=y_{t}-\hat{\mathrm{y}}_{t}
$$

\subsubsection{Detecting deforestation from Landsat NDVI time series}

We also detected deforestation from Landsat NDVI time series following the same approach presented in Section 2.4.5, except that here we updated the regression equation to include the seasonal terms to account for seasonal variations in NDVI. The regression equation that includes the seasonal terms takes the form:

$$
\hat{\mathrm{y}}_{t}=\beta_{0}+\sum_{j=1}^{k} \gamma_{j} \sin \left(\frac{2 \pi j t}{f}+\delta_{j}\right)+\varepsilon_{t}
$$

where $\beta_{0}$ is the y-intercept coefficient, $\gamma$ is the amplitude, $\delta$ is the phase of the harmonic season and $\varepsilon_{t}$ is the random error at time $t$ (Verbesselt, Hyndman, Zeileis, et al. 2010; Verbesselt, Hyndman, Newnham, et al. 2010; Verbesselt et al. 2012). We set the frequency $f$ to 23 , corresponding to the number of images that would be acquired at each location by a Landsat sensor per year based on a 16 -day revisit frequency. We fitted three harmonic terms (i.e. $\mathrm{k}=3$ ). As in Section 2.4.5, we calculated the magnitude of change $\left(m c_{\rho}\right)$ using Eq.2.5.

\subsubsection{Collecting validation data}

In many parts of the globe, reliable validation data for forest change are lacking. As a result, high and medium spatial resolution satellite data are mostly used as source of validation data (Cohen et al. 2010; Zhu \& Woodcock 2014b; Dutrieux et al. 2015). Here, we also collected validation data for deforestation from Landsat images, complemented by high spatial resolution imagery available in Google Earth (https://www.google.com/earth/) and Bing Maps (http://www.bing.com/maps/), through visual image interpretation. We defined deforestation as loss of forest cover that is visually visible from Landsat images and other high resolution imagery available in Google Earth and Bing Maps. To facilitate the collection of validation data, forest and deforested areas were first digitised from Landsat images at each site, resulting into two strata per study site: forest and deforested stratum. Deforested stratum consists of all areas which were deforested in 2005 
through 2014, whereas the forest stratum consists of areas which were still forest at the end of 2014. In total, 550 sample points we generated at the Bolivian site and 470 at the Brazilian site. In each stratum, we generated sample points proportionate to the area of the stratum, using probability sampling. For each study site, a training data set, $15 \%$ of the sample points, was sampled from these sample points also using proportional probability sampling. The training data set was used to validate the results generated during the calibration of BFAST algorithm (Section 2.4.4). The remaining validation data $(85 \%)$, the test data set, were used to validate the final deforestation maps produced after calibration of BFAST algorithm. For each sample point in deforested stratum, the date for deforestation was documented by systematically tracing the first image on which the deforestation event was visible. The acquisition date of the image in which deforestation event was first visible was used as a surrogate date for when the deforestation has occurred, and such date was used to the calculate the temporal detection delay. The procedure followed here to collect validation data is consistent with approaches recommended or used in previous studies (Cohen, Yang, \& Kennedy, 2010; Olofsson et al., 2014; Zhu \& Woodcock, 2014b).

\subsubsection{Accuracy assessment}

We estimated the overall spatial accuracy, commission and omission errors, bias and temporal detection delay for deforestation detected from NDVI and sNDVI using test validation data set (Table 2.1). The bias is the difference between commission and omission error. The spatial accuracy was calculated based on the overall change detected at each study site in 2005 through 2014. Confidence interval for the overall spatial accuracy was calculated using binomial probability of success using Wilson's method (Wilson 1927). In some cases, a change was detected at locations where deforestation has indeed occurred, but the change is detected before the deforestation has occurred. Such scenario occurs when not all remnant noise is successfully removed from the time series. If such incidents are not taken into account, estimated overall spatial accuracy would be falsely amplified. In our analysis, we treated such incidents as commission error.

The temporal detection delay was determined at each sample point by counting the number of valid observations available between the image in which deforestation was visually identified, and the image in which deforestation was detected by BFAST. The temporal detection delay was calculated only for sample points where the date of deforestation detected from NDVI or sNDVI was similar to, or later than, that of the validation data. We expressed the temporal detection delay in the number of observations as unit of measurement, and not in time, because expressing temporal detection delay in terms of observations provides a clear picture on how robust a 
change detection method is, and it also makes it possible to clearly see the number of observations required before deforestation is detected.

We used a paired t-test to compare the temporal detection delay for deferent spatial window sizes which were used to calculate the sNDVI. The paired $t$-test was also used to compare the temporal detection delay achieved from NDVI (seasonal model approach) to the temporal detection delay achieved from sNDVI (spatial context approach). For the paired t-test, a 5\% significant level was used as threshold to accept or reject the null hypothesis that the difference in temporal detection delays is significant.

Finally, to demonstrate that it is possible to monitor deforestation throughout the year in the tropics using Landsat image time series, we plotted a graph showing distribution of the date of deforestation based on validation data, and changes detected from sNDVI and NDVI.

\section{$2.5 \quad$ Results}

\subsubsection{Spatial context approach vs. seasonal model approach}

Reducing seasonal variations in the Landsat image time series using spatial context (spatial window $=61 \times 61$ pixels) when detecting deforestation resulted in the overall spatial accuracy higher than when a seasonal model was used (Figure 2.6a,b). The overall spatial accuracy of $95.9 \%$ was achieved at the Bolivian site when spatial context was used to reduce seasonal variations in Landsat image time series, whereas an overall spatial accuracy of $94.0 \%$ was achieved when a seasonal mode was used. Conversely, at the Brazilian site, the overall spatial accuracy of $85.5 \%$ was achieved when spatial context was used to account for seasonality, whereas $82.5 \%$ overall spatial accuracy was achieved when a seasonal model was used. The difference (Bolivian site $=1.9 \%$, Brazilian site $=3.0 \%$ ) in the overall spatial accuracy observed between the spatial context and seasonal model approach at each site was marginal. The incidents of false detections were lower at the Bolivian site when spatial context was used to reduce seasonal variations in Landsat image time series than when a seasonal model is used to account for seasonality (Figure 2.6a). At the same site, deforestation events were omitted almost equally when either spatial context or seasonal model was used to reduce seasonal variations. In contrast, at the Brazilian site, incidents of detecting false detections when spatial context was used to reduce seasonal variations in Landsat image time series were almost equal to those of when a seasonal model was used to account for seasonality (Figure 2.6b). Unlike at the Bolivian site, the incidents of omitting deforestation events were higher when a seasonal model was used to account for seasonality in Landsat image time series at the Brazilian site than when spatial context was used to reduce seasonal variations (Figure 2.6b). Figure 2.7 shows deforestation events detected at each study site from the time series of spatially normalised 
Landsat vegetation index (sNDVI; the spatial context approach), and Landsat normalised difference vegetation index (NDVI; the seasonal model approach). The benefit of using spatial context to reduce seasonal variations in Landsat image time series when mapping deforestation was more pronounced in the temporal detection delay, especially at the Bolivian site (Figure 2.6c). The mean temporal detection delay for deforestation events at the Bolivian site when spatial context was used to reduce seasonal variations in Landsat image time series, prior to deforestation mapping, was shorter than when a seasonal model was used to account for seasonality $(\mathrm{t}=2.3855$, df $=15, \mathrm{p}<$ 0.05 ). The median temporal detection delay from the spatial context approach was two observations, seven times shorter than the median temporal detection delay from seasonal model approach ( 15 observations) at the Bolivian site. The temporal detection delay achieved from spatial context and seasonal model approach were not significantly different at the Brazilian site $(\mathrm{t}=1.7703, \mathrm{df}=57, \mathrm{p}>0.05)$. 

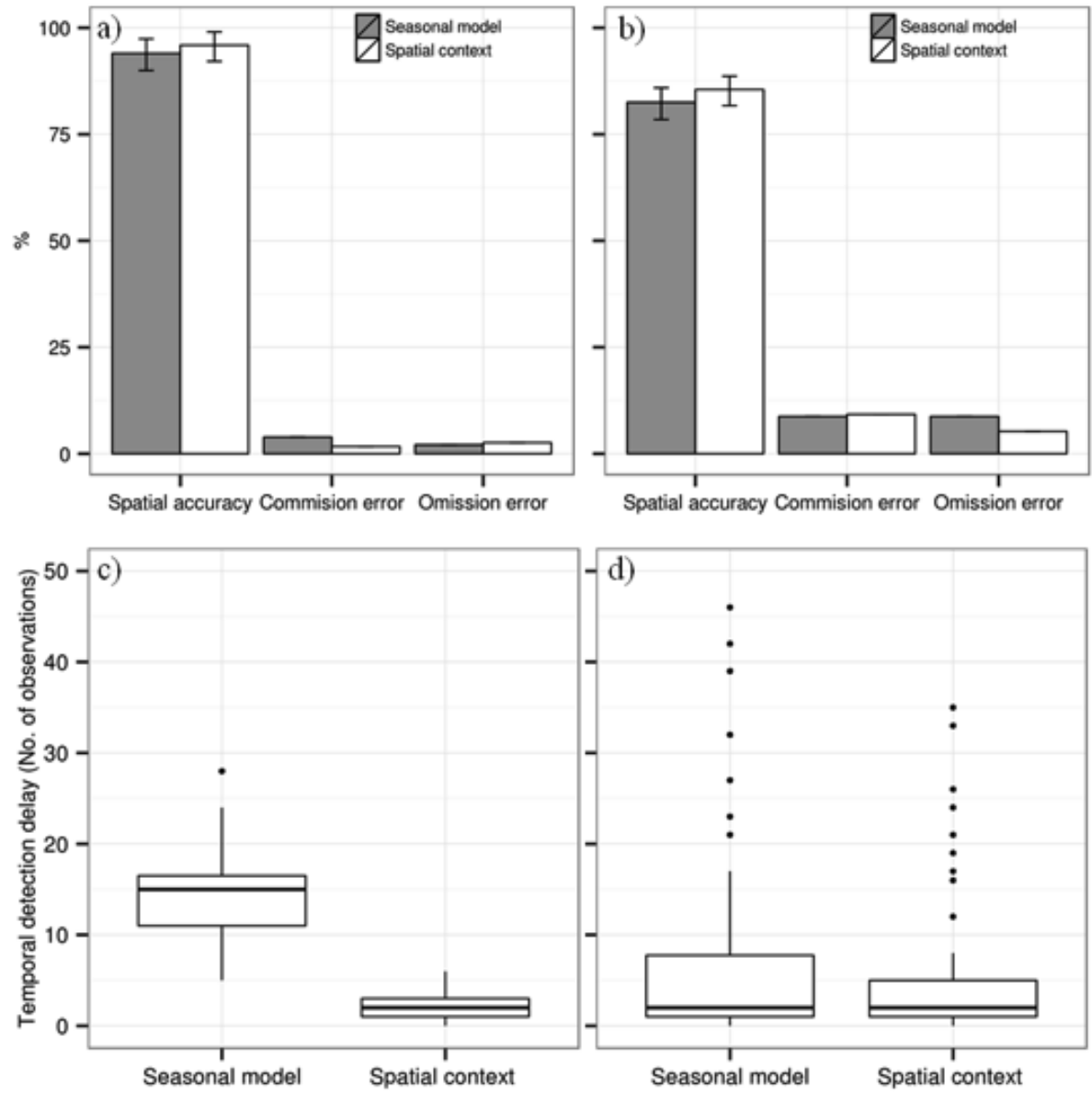

Figure 2.6: Overall spatial accuracy $(\mathrm{a}, \mathrm{b})$ and distribution of temporal detection delay (c, d) for deforestation events detected from spatially normalised vegetation index (sNDVI) and Landsat normalised difference vegetation index (NDVI) time series at the Bolivian (a, c) and Brazilian (b, d) study sites. Deforestation is for the period of 2005 through 2014, and was detected using BFAST. The "error" bars in (a) and (b) are 95\% confidence intervals for the overall spatial accuracy, calculated using binomial probability of success based on Wilson's method (Wilson, 1927). The black dots in (c) and (d) represent the outliers. 


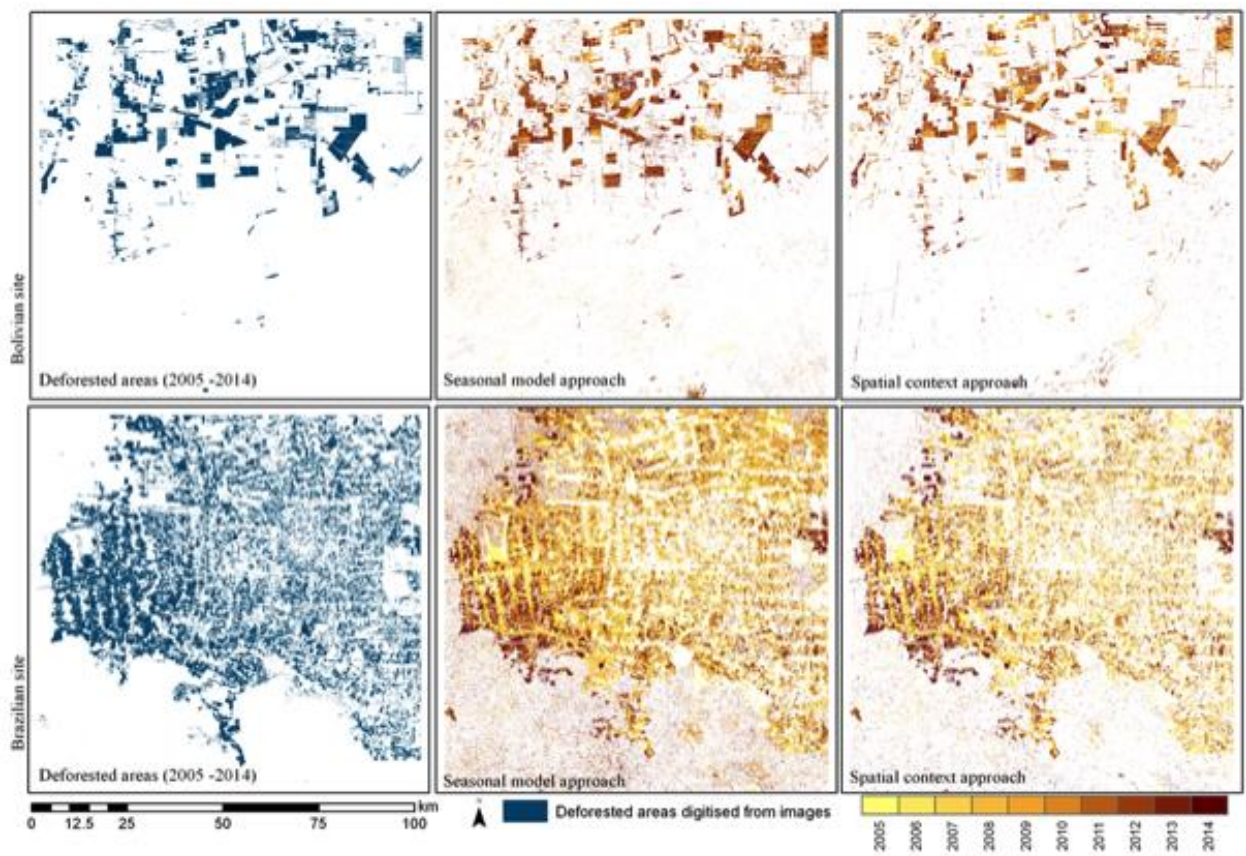

Figure 2.7: Deforestation events detected from the time series of spatially normalised Landsat vegetation index (sNDVI; the spatial context approach), and Landsat normalised difference vegetation index (NDVI; the seasonal model approach), at the Bolivian and the Brazilian study site. Deforestation is for the period of 2005 through 2014, and was detected using Breaks For Additive Season and Trend (BFAST) method. Deforested area data in the first column were digitised from Landsat images.

\subsubsection{Effect of spatial window size on spatial accuracy}

Mapping deforestation from sNDVI time series calculated from each spatial window resulted in the overall spatial accuracy above $92 \%$ at the Bolivian site, and above $85 \%$ at the Brazilian site. For the Bolivian site, the highest overall spatial accuracy $(95.9 \%)$ was achieved from sNDVI time series calculated from the spatial window of 334.89 ha (W61), and the lowest overall spatial accuracy (92.1\%) was achieved from the spatial window of 1317.69 ha (W121) (Figure 2.8a). The spatial window (W61) that achieved the highest overall spatial accuracy at the Bolivian site also achieved the lowest bias ($0.7 \%)$. Apart from window W9, W101, W121, the bias at the Bolivian site was in favour of the omission error (Figure 2.8b), implying that for these windows the omission error was higher than the commission error. At the Brazilian site, the highest overall spatial accuracy (89.0\%) was achieved from sNDVI time series calculated using a spatial window of 56.25 ha (W25), whereas the 
lowest overall spatial accuracy (85.5\%) was achieved from the spatial window of 334.89 ha (W61).Like at the Bolivian site, the spatial window (W25) that achieved the highest spatial accuracy at Brazilian site also achieved the lowest bias (1.7\%). Unlike at the Bolivian site, the bias for each spatial window at the Brazilian site was in favour of the commission error, implying that for all windows the commission error was higher than the omission error. At the Brazilian site, smaller spatial windows (W9, W25, W35 and W41) achieved higher overall spatial accuracy than larger windows (W61, W81, W101, and W121).
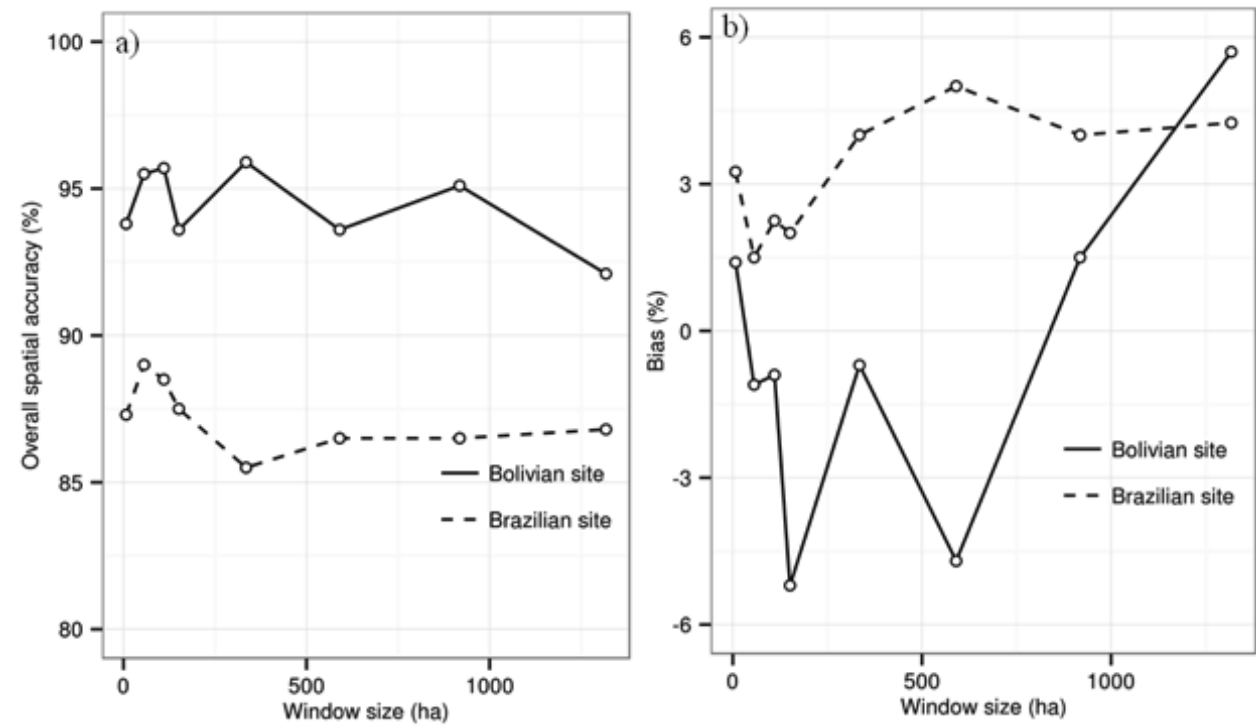

Figure 2.8: (a) overall spatial accuracy for deforestation detected from the spatially normalise difference vegetation index (sNDVI) at the Bolivian and Brazilian study sites; and (b) the bias between commission and omission error (Bias $=$ commission error - omission error $)$. Varying spatial window sizes were used to calculate sNDVI.

\subsubsection{Effect of spatial window size on temporal detection delay}

The temporal detection delay for deforestation events at the Bolivian site (Figure 2.9a) was not different across the spatial windows except for W61 which recorded a shorter mean temporal detection delay than window $\mathrm{W} 101(\mathrm{t}=-2.4227 \mathrm{df}=15, \mathrm{p}<0.05)$. For the Brazilian site (Figure 2.9b), the mean temporal detection delay for W61 was shorter than that of W9 $(\mathrm{t}=$ 2.1914, df $=57, \mathrm{p}<0.05), \mathrm{W} 25(\mathrm{t}=2.2629, \mathrm{df}=57, \mathrm{p}<0.05)$ and W35 $(\mathrm{t}=$ $2.0867, \mathrm{df}=57, \mathrm{p}<0.05)$. The mean temporal detection delays for all other windows were not different at the Brazilian site. A median temporal detection delay of two observations was achieved when detecting deforestation from 
sNDVI calculated using each of the spatial windows at the Bolivian site. For the Brazilian site, a median temporal detection day of one observation was achieved from W25, whereas a median temporal detection delay of two observations was achieved from W35, W41, W61, W81, W101, and W121. The smallest window (W9) achieved a median temporal detection delay of four observations at the Brazilian site. Generally, the sNDVI time series calculated from W61 resulted in shorter mean temporal detection delay at each study site.
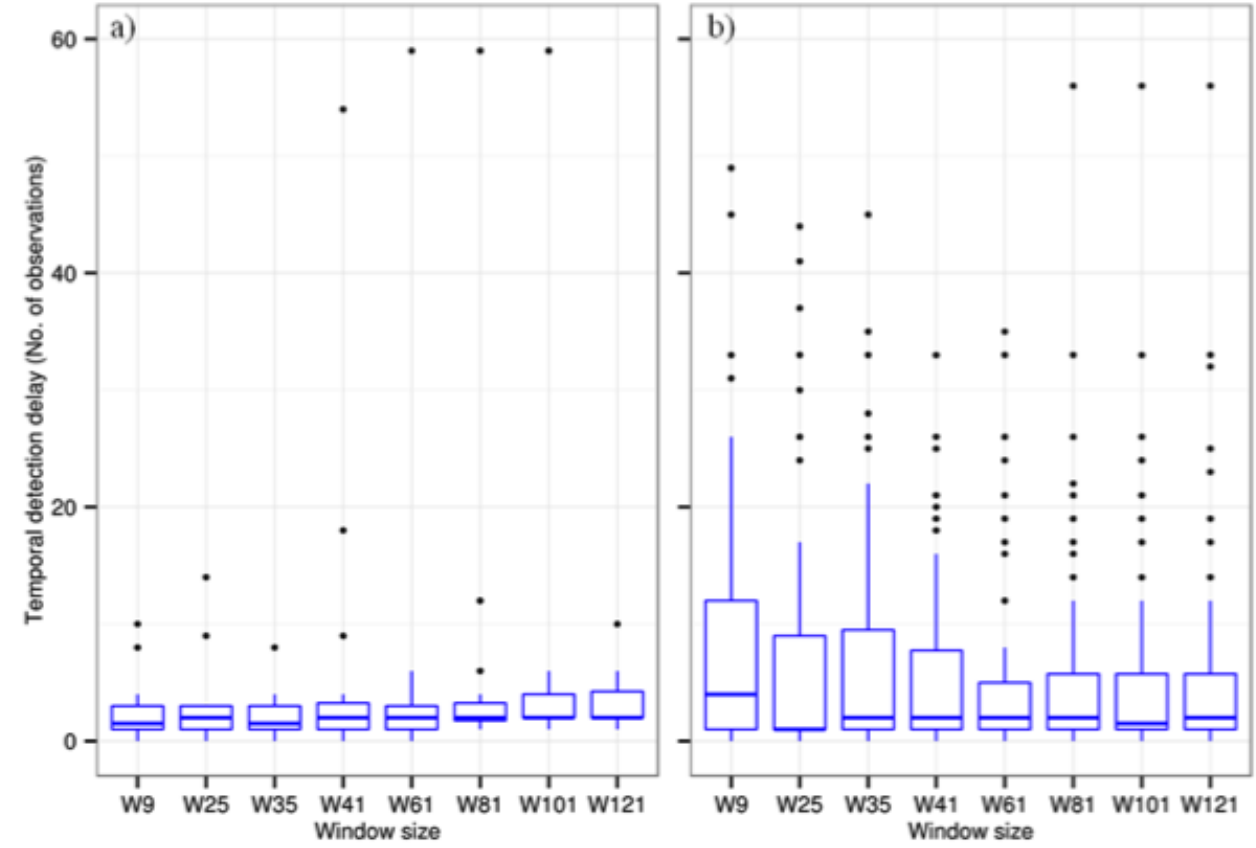

Figure 2.9: Distribution of deforestation temporal detection delay per spatial window size for (a) the Bolivian and (b) Brazilian study site. Deforestation was detected from spatially normalised deference vegetation index (sNDVI) calculated from Landsat normalised deference vegetation index (NDVI). The black dots are outliers.

\subsubsection{Effect of spatial window size on computational time}

Increasing the size of the spatial window resulted in a linear increase in the computational time per pixel when computing sNDVI (Figure 2.10). When using $\mathrm{R}$ software ( $\mathrm{R}$ Core Team, 2014) on a LINUX computer with 32 GB RAM, the computational time per pixel increased from 0.000318 seconds for the smallest window (W9) to 0.001755 seconds for the largest window (W121). 


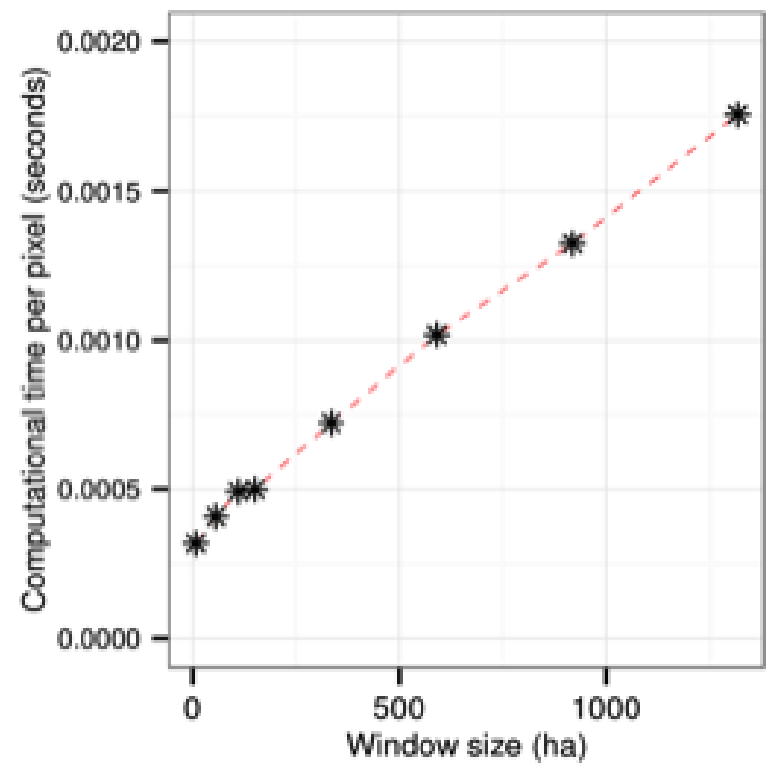

Figure 2.10: Computational time per pixel for each spatial window when calculating spatially normalised difference vegetation index (sNDVI) from Landsat normalised deference vegetation index (NDVI).

\subsubsection{Effect of magnitude threshold on spatial accuracy and temporal detection delay}

Increasing the magnitude threshold for deforestation, in absolute term, leads to an increase in the overall spatial accuracy, but only until the maximum overall spatial accuracy is reached (Figure 2.11a, b). After the maximum overall spatial accuracy is reached, increasing the magnitude threshold further leads to a decrease in overall spatial accuracy. At smaller magnitude thresholds, the bias is in favour of false detections, and it favours omission of deforestation events at larger magnitude thresholds (Figure $2.11 \mathrm{a}, \mathrm{b})$. At larger magnitude thresholds, omission of deforestation was much higher at the Brazilian site than at the Brazilian site (Figure 2.11a, b). Generally, the median temporal detection delay was higher at lower magnitude thresholds for the Bolivia site, and lower for the Brazilian site (Figure 2.11c, d). 

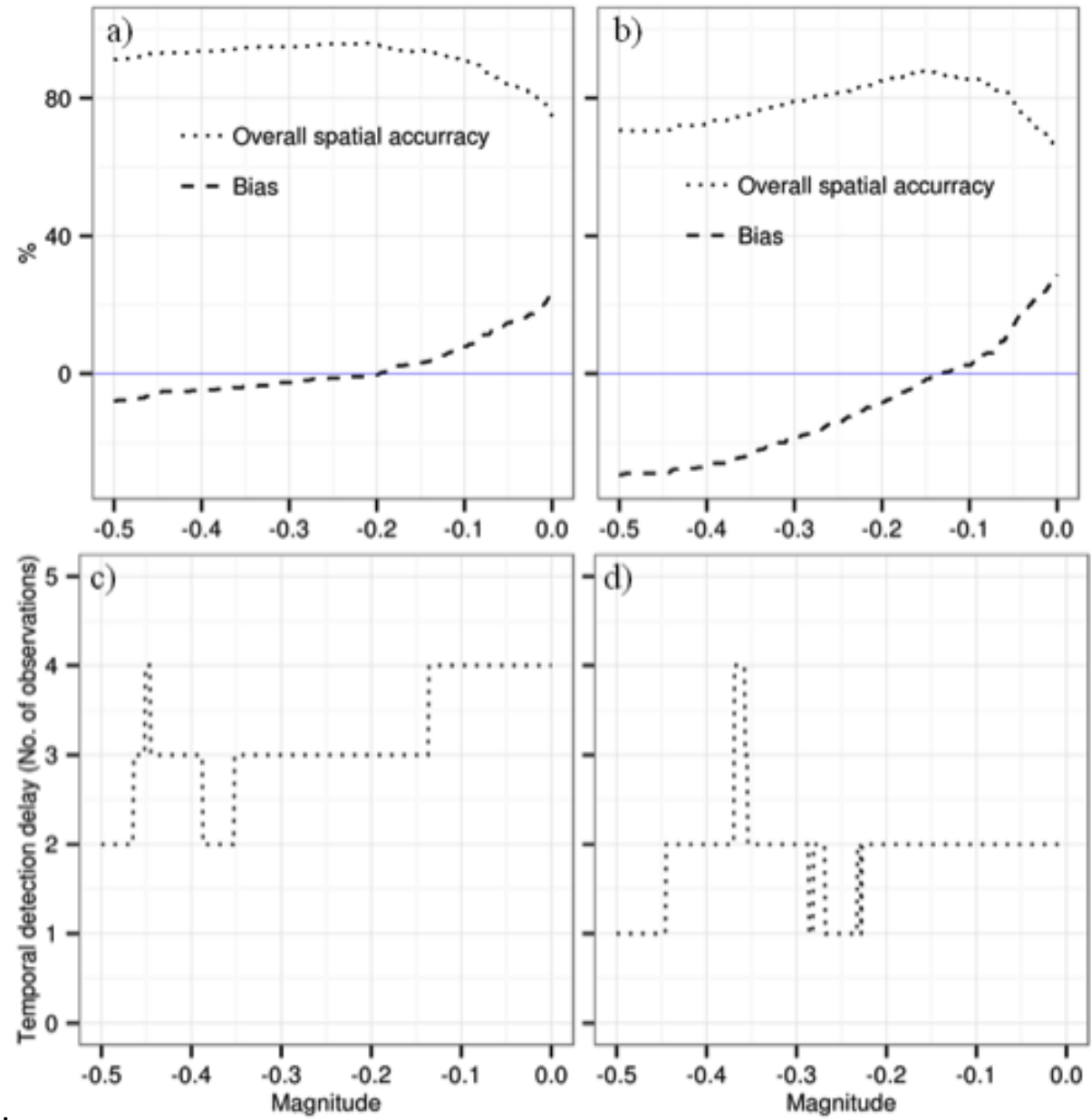

Figure 2.11: An example of how the overall spatial accuracy, area bias and temporal detection delay change when using different magnitude thresholds to discriminate deforestation from other disturbances. Graphs on the left (a and c) are for Bolivian site, and the graphs on the right (b and c) are for the Brazilian site.

\subsubsection{Monitoring deforestation with Landsat data throughout the year}

There was a valid Landsat image in each month at each study site during the period of 1984 through 2014 (Figure 2.12a). In general, valid images were distributed almost equally among the months at the Bolivian site, whereas most of the valid images at the Brazilian site were mainly available during the month of April -October. Our validation data show that deforestation occurred at the Bolivian site throughout the year (Figure 2.12b, 
c), whereas deforestation was mainly restricted to the period of April October at the Brazilian site. Generally, the day-of-the-year for deforestation events detected either from NDVI or sNDVI exhibits temporal pattern similar to that of validation data (Figure 2.12b, c), but at each site the distribution of the day-of-the-year for deforestation detected from NDVI was biased more to the months of September and October than for deforestation detected from sNDVI.
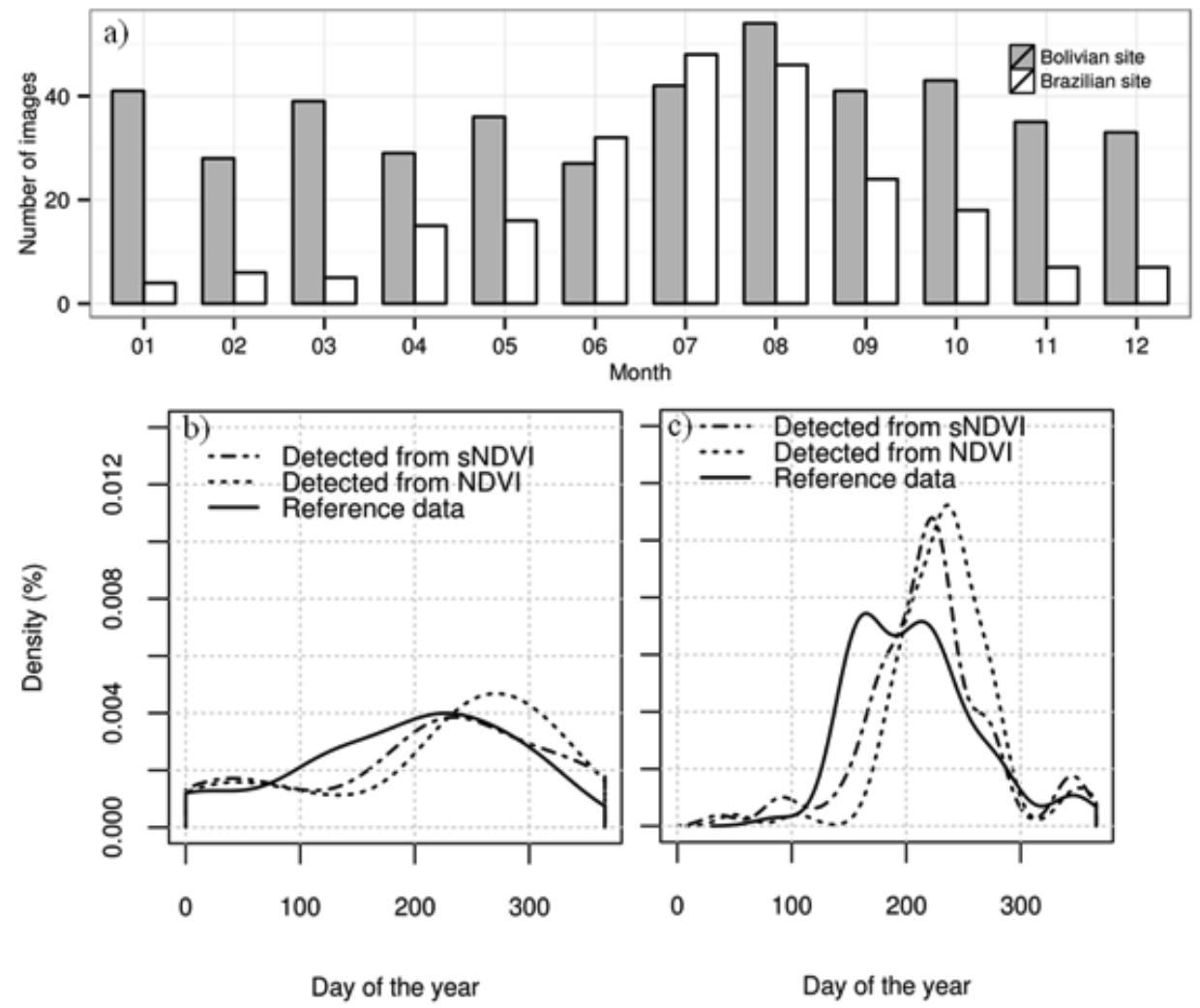

Figure 2.12: (a) Number of Landsat images per month acquired by Landsat Thematic Mapper (TM 5) and Enhanced Thematic Mapper Plus (ETM+ 7) for a period of 1984 through 2014, and (b, c) the distribution of day-of-the-year (DOY) for deforestation events derived from validation data and deforestation detected from nor normalised deference vegetation index (NDVI) and spatially normalised deference vegetation index (sNDVI) at the (b) Bolivian and (c) Brazilian site. 


\subsection{Discussion}

In this chapter, we propose a new approach for reducing seasonal variations in satellite data using spatial context, and evaluate whether reducing seasonal variations in satellite data using spatial context improves early deforestation detection by comparing our approach to a seasonal modelbased approach. We demonstrated the spatial context approach on Landsat data at dry (Bolivian site) and evergreen (Brazilian site) forest site. Our results show that deforestation events are detected earlier if we reduce seasonal variations in the data using spatial context than when we use seasonal model. The benefit of reducing seasonal variations in Landsat image time series using spatial context is only significant at the Bolivian site where the forests exhibit strong seasonality. At this site, we were able to achieve a median temporal detection delay of two observations, which is seven times shorter than if we use the seasonal model (15 observations). This significant decrease in the temporal detection delay when mapping deforestation from spatially normalised Landsat time series can be attributed to the fact that the spatial context approach minimises the fluctuations in the time series while amplifying the impact of a disturbance. Such amplification of the disturbance impact enabled the MOSUM of residuals (Chu et al. 1996; Zeileis et al. 2005) to quickly cross its critical boundary. BFAST, which we used to detect deforestation, only detects abrupt changes (breakpoints) in the time series if the MOSUM of residuals crosses its critical boundary. Therefore, shorter temporal detection delay indicates that the MOSUM of residuals was crossing its critical boundary earlier if we use spatial context to reduce seasonal variations than when using a seasonal model. Fitting a seasonal model is difficult when there are not enough temporal observations. Model overfitting is likely to occur, and this may affect the removal of seasonality. If seasonality is not properly removed, the impact of a deforestation event can be disguised by seasonality, and this may lead to a delay in detecting deforestation.

Unlike at the Bolivian site, no significant difference in the temporal detection delay was observed between the spatial context and seasonal model approach at the Brazilian site. This non-significant difference in temporal detection delay at the Brazilian site can be explained by inherently low seasonality in the time series at this site. The forest at this site is evergreen, and the impact of deforestation on the time series can hardly be disguised by seasonal variations. As a result, the impact of deforestation on either original or spatially normalised Landsat time series is therefore likely to immediately push the MOSUM of residuals beyond its critical boundary at evergreen forest sites.

The overall spatial accuracy achieved from the spatial context approach at each study site was marginally higher than the one achieved from the seasonal model approach. At the Bolivian site, the overall spatial accuracy 
from the spatial context approach was higher than that of the seasonal model approach because there were less false detection (commission error) when seasonality was reduced using spatial context. Possibly, the seasonal model was not effective in accounting for seasonality, and some seasonal variations might have been detected as deforestation at the Bolivian site, thus resulting in higher commission error. Another possible explanation for why commission error from seasonal model was higher than that from spatial context at the Bolivian site could be drought effects. Unlike seasonal model, spatial context has potential to remove the impact of drought disturbances from the time series. Such drought disturbances could be lead to false alarms of deforestation if not removed from the time series. The overall spatial accuracy from the spatial context approach at the Brazilian site was mainly higher than that of the seasonal model approach because the incidents of omitting deforestation (omission error) were few when seasonality was reduced using spatial context. A possible explanation for low omission error from spatial context at the Brazilian site than that from seasonal model is the amplification of the impact of deforestation on the time series. Spatial context approach normalise the value of the focal pixel, and this procedure has potential to amplify the impact of local disturbances on the time series. Omission error from the spatial context approach was therefore lower than that from the seasonal model approach possibly because the impact of deforestation on the time series was amplified, making it easier for the BFAST to distinguish deforestation from normal forest dynamics.

The overall spatial accuracy achieved in this study either from spatial context or seasonal model approach is consistent with accuracies achieved from other studies that used Landsat image time series to map deforestation (Devries, Verbesselt, et al. 2015; Dutrieux et al. 2015; Zhu \& Woodcock 2014b; Reiche, de Bruin, et al. 2015; Reiche, Verbesselt, et al. 2015). For temporal detection delay, previous studies (Reiche, Verbesselt, et al. 2015; Reiche, de Bruin, et al. 2015; Dutrieux et al. 2015), which looked at the temporal detection delay experienced when mapping deforestation from Landsat time series, expressed the temporal detection delay in time. Here, we used the number of observations as a measure for temporal detection delay. It is therefore not possible to compare the temporal detection delay achieved in this study to that of other studies. Using time as measure of temporal detection delay is intuitive but such approach makes it difficult to understand why a delay is experienced. In time, a delay can be caused by the change detection algorithm, but lack of observations because of cloud cover can also lead to a delay, time-wise.

Deforestation events were detected with higher spatial accuracy at the Bolivian site than at the Brazilian site. These differences could be explained by the process causing deforestation. At the Bolivian site, deforestation is dominated mainly by industrial agricultural expansion with large blocks of 
deforestation events whereas at the Brazilian site deforestation events are heterogeneous and correspond mostly to a process of colonization. Large blocks of deforestation events may be easier to detect than deforestation caused colonization because such changes are typically abrupt. There were fewer images at the Brazilian site than at the Bolivian site. This difference in temporal distribution of available images might have also contributed to the inter-site difference in overall spatial accuracy. Detectability of deforestation events is likely to increase with the number of images available at the study site.

Landsat NDVI images were used in this study, but our spatial context approach can be applied to any satellite-derived vegetation index, and is also not restricted to Landsat data. Although we used 90 th percentile when calculating sNDVI, other percentiles can equally be used but selecting lower percentiles (e.g. 50 th) can potentially lead to lower $\mathrm{VI}_{\text {median }}$ which may undesirably smooth out the impact of deforestation from the time series. Furthermore, the results presented in this study only came from two sites which are in the same continent. Therefore, the size of spatial windows used here to calculate sNDVI, and the magnitude thresholds we applied, may not be optimal in other parts of the globe where different pattern of deforestation exist. For example, in Ethiopia, forest change processes occur at small-scale and the change is generally gradual (Devries, Verbesselt, et al. 2015). Such forest disturbances may be detected more accurately if a different window size or magnitude threshold is applied. Further work is therefore needed in many parts of the globe in order to determine more robust spatial window size(s) from which sNDVI can be calculated, and also to rigorously calibrate BFAST algorithm.

We have shown that reducing seasonal variations in Landsat data using spatial context when mapping deforestation is beneficial in forests that exhibit strong seasonality. Strong seasonality is a major challenge when mapping deforestation at sub-annual scales from Landsat data. Therefore, our spatial context approach is a novel approach that provides a unique opportunity to map deforestation from Landsat data at sub-annual scales in areas where forests exhibit strong seasonality. However, our spatial context approach may face challenges in heterogeneous forests, where evergreen and deciduous are mixed. In heterogeneous forest, calculating $\mathrm{VI}_{\text {median }}$ from pixels above $90^{\text {th }}$ percentile may not reduce seasonal variations for pixels, which are deciduous because the all pixels above $90^{\text {th }}$ percentile might be from evergreen pixels. A possible solution for mixed forests when using spatial context is to normalise the value of the focal pixel with $\mathrm{VI}_{\text {median }}$ calculated from neighbouring pixels, which show similar temporal variations to that of focal pixel. In this way, we can avoid normalising a deciduous forest pixel with $\mathrm{VI}_{\text {median }}$ calculated from evergreen forest pixels. Identifying pixels that show temporal dynamics similar to that of the focal pixel is likely to be a 
cumbersome step and computationally demanding, and may make the spatial context approach less attractive.

Our spatial context approach adds extra computational time to already computationally demanding analysis of satellite data. This shortcoming is even more pronounced if larger spatial window are used when computing sNDVI. Fortunately, our results show that even if smaller spatial windows, which are less computationally demanding, are used, deforestation can still be detected much earlier with high overall accuracy than when a seasonal model is used.

The spatial context approach we propose here can also be applied on highly irregular image time series like those from RapidEye and Disaster Monitoring Constellation (DMC) satellite sensors to remove seasonality effects in order to enable change detection at high spatial resolution.

\subsection{Conclusion}

In this chapter, we presented a new approach that reduces seasonality in Landsat data using spatial context, and compared this new approach to a seasonal model approach. We have demonstrated that deforestation can be detected with a short delay even in the tropical dry forests if we use spatial context to reduce seasonal variations in the data. A median temporal detection delay of two observations was achieved in forest with strong seasonality from spatial context approach, and this delay was seven times shorter than if we use the seasonal model (15 observations). In the humid tropical forest, the difference in the temporal detection delay between spatial context and seasonal model approach was not significant. The gain in the overall spatial accuracy is only marginally higher when using spatial context than when using a seasonal model. The spatial context approach we presented in this chapter is a novel and useful approach for timely detection of deforestation events in areas where forests exhibit strong seasonality and it provides opportunity to detect deforestation events from satellite data throughout the year even in deciduous forests. Therefore, our spatial context approach provides unique opportunity to monitor deforestation events in dry tropical forests at sub-annual scales using Landsat data. With data from sentinel constellation becoming available it will be possible to detect deforestation events within days or weeks of occurrence. 


\section{Acknowledgements}

This work was funded through Google Deforestation Monitoring project (grant agreement 5120906), the European Union Seventh Framework Programme (FP7/2007-2013; grant agreement Number 283093) - The Role of Biodiversity in Climate Change Mitigation (ROBIN), and European Space Agency (ESA) ForMoSa- Forest Degradation Monitoring with Satellite Data Project (grant agreement 5160957022). 


\section{Chapter}

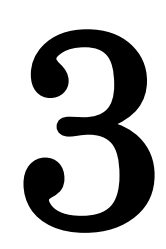

\section{Monitoring deforestation at sub-annual scales as extreme events in Landsat data cubes}

This chapter is based on:

Hamunyela, E., Verbesselt, J., Bruin, S. De, \& Herold, M. (2016). Monitoring deforestation at sub-annual scales as extreme events in Landsat data cubes. Remote Sensing, 8, 651. 


\begin{abstract}
Current methods for monitoring deforestation from satellite data at subannual scales require pixel time series to have many historical observations in the reference period to model normal forest dynamics before detecting deforestation. However, in some areas, pixel time series often do not have many historical observations. Detecting deforestation at a pixel with scarce historical observations can be improved by complementing the pixel time series with spatial context information. In this work, we propose a data-driven spacetime change detection method that detects deforestation events at sub-annual scales in data cubes of satellite image time series. First we spatially normalised observations in the local space-time data cube to reduce seasonality. Subsequently, we detected deforestation by assessing whether a newly acquired observation in the monitoring period is an extreme when compared against spatially normalised values in a local space-time data cube defined over reference period. We demonstrated our method at two sites, a dry tropical Bolivian forest and a humid tropical Brazilian forest, by varying the spatial and temporal extent of data cube. We emulated a "near real-time" monitoring scenario, implying that observations in the monitoring period were sequentially rather than simultaneously assessed for deforestation. Using Landsat normalised difference vegetation index (NDVI) time series, we achieved a median temporal detection delay of less than three observations, a producer's accuracy above 70\%, a user's accuracy above 65\%, and an overall accuracy above $80 \%$ at both sites, even when the reference period of the data cube only contained one year of data. Our results also show that large percentile thresholds (e.g., $5^{\text {th }}$ percentile) achieve higher producer's accuracy and shorter temporal detection delay, whereas smaller percentiles (e.g., 0.1 percentile) achieve higher user's accuracy, but longer temporal detection delay. The method is data-driven, not based on statistical assumption on the data distribution, and can be applied on different forest types. However, it may face challenges in mixed forests where, for example, deciduous and evergreen forests coexist within short distances. A pixel to be assessed for deforestation should have a minimum of three temporal observations, the first of which must be known to represent forest. Such short time series allow rapid deployment of newly launched sensors (e.g., Sentinel-2) for detecting deforestation events at sub-annual scales.
\end{abstract}

Keywords: Landsat, deforestation, spatial context, seasonality, seasonal model 


\subsection{Introduction}

Monitoring deforestation at sub-annual scales (e.g., weekly or monthly) using satellite data is increasingly becoming an important part of the initiatives that aim to reduce deforestation across the globe. This is because monitoring deforestation at the sub-annual scale, unlike annual monitoring, allows for timely detection of deforestation events, thus providing an opportunity for early interventions to stop illegal deforestation activities (Shimabukuro et al. 2006). For example, the Brazilian Institute for Space Research (INPE) monitors deforestation events at sub-annual scales in the Amazon using a system based on Moderate Resolution Imaging Spectroradiometer and bi-temporal change detection which has played an important role in reducing deforestation in Brazil. However, forest monitoring systems which detect deforestation events at sub-annual scales based on a bitemporal change detection approach may face challenges in areas where forest has strong seasonality. To address this challenge, methods that detect deforestation at sub-annual scales from satellite image time series while accounting for seasonal variations have been developed in recent years (Hamunyela, Verbesselt \& Herold 2016; Hansen et al. 2016; Hilker, Wulder, Coops, Linke, et al. 2009; Zhu \& Woodcock 2014b; Verbesselt et al. 2012; Dutrieux et al. 2015; Reiche, de Bruin, et al. 2015). These methods detect deforestation events by testing if a newly acquired observation at a particular pixel is abnormally low when compared to historical temporal dynamics of forest at such pixel (Verbesselt et al. 2012; Reiche, de Bruin, et al. 2015; Zhu \& Woodcock 2014b; Reiche, Verbesselt, et al. 2015). However, for the test to be robust, a pixel time series is required to have many historical observations. In some areas, however, pixel time series often do not have enough historical observations, mainly because of persistent cloud cover coupled with a relatively long revisit time in the past (Asner 2001), especially for satellite sensors which have high and medium spatial resolutions. To remedy the problem of cloud cover, new methods that detect deforestation events at sub-annual scales by combining optical and synthetic aperture radar (SAR) data have been proposed (Reiche 2015; Reiche, Verbesselt, et al. 2015). However, these methods also require a pixel to have many historical observations. In the past, SAR sensors, which can penetrate the clouds, had limited temporal coverage, also resulting in sparse time series. Such sparse historical observations make it difficult to properly model the normal temporal dynamics of the forest, and may lead to many false detections during deforestation detection. In the near future, however, temporally dense SAR time series from Sentinel sensors will be available, and such dense time series, when combined with data from optical sensors, will address some of the current challenges associated with monitoring deforestation at sub-annual scales in the tropics (Reiche et al. 2016). In particular, it will be possible to detect deforestation events within a few days of occurrence. However, with such dense time series, another 
challenge related to pre-processing huge amounts of historical data will arise: pre-processing huge amounts of historical data for large areas is likely to take a relatively long time, thus affecting rapid detection of deforestation events.

The challenge associated with monitoring deforestation at sub-annual scales in areas with scarce historical observations can also be addressed by exploiting both temporal and spatial information in satellite image time series. An individual pixel may not have enough observations, but the spatial context derived from neighbouring pixels can provide sufficient information to determine whether a pixel with scarce historical observations is deforested or not. Such an approach may reduce the amount of historical satellite data that needs to be processed when monitoring deforestation at sub-annual scales.

Recently, Huang and colleagues (Huang et al. 2009) exploited spatiotemporal Landsat data to identify deforested areas, but that work focused on detecting deforestation at the annual scale. Similarly, Hamunyela and co-workers (Hamunyela, Verbesselt \& Herold 2016) used the spatial context of pixels to reduce seasonal variations in Landsat time series before detecting deforestation at sub-annual scales using a method (Verbesselt et al. 2012) which relies on individual pixel time series. These studies demonstrate that spatiotemporal information of image time series is useful for deforestation monitoring. However, methods for detecting deforestation at sub-annual scales based on integrated analysis of spatiotemporal data have not been published, to the best of our knowledge.

The approach proposed in this chapter is to identify deforested pixels by exploiting spatiotemporal information available in the space-time data cube of satellite image time series. In this way, a pixel with at least three temporal observations in its time series is expected to still allow assessment of deforestation at sub-annual scales. This is because observations in the spacetime data cube are likely to be sufficient for deciding whether a newly acquired observation is abnormally low. However, shifting to space-time change analysis requires two major challenges to be overcome. The first challenge concerns dealing with seasonality in the data cube. Seasonal variations may disguise deforestation in the data if not removed, especially in dry forests where seasonality is strong. The second challenge is how to consistently identify anomalies in a space-time data cube. Existing methods for identifying anomalous observations in space-time data cubes of climatic data (Davis \& Mikosch 2008) and global gross primary production (Zscheischler et al. 2013) nevertheless focus on the temporal perspective, since thresholds are defined without considering spatial context.

This chapter describes a data-driven space-time change detection method for monitoring deforestation at sub-annual scales. The method detects deforestation at pixel level as an extreme event in vegetation index values within local space-time cubes of satellite image time series. With this method, spatial-temporal information in satellite image time series is exploited to 
detect deforestation at sub-annual scales. Our method builds upon the spatial context approach developed in a previous study (Hamunyela, Verbesselt \& Herold 2016) for reducing seasonal variations in satellite image time series. The method we propose here demonstrates how data cubes of satellite image time series can be used for sub-annual deforestation monitoring. We demonstrated our method at two sites, a dry tropical forest and a humid tropical forest (Section 3.2.3), using normalised difference vegetation index (NDVI, (Rouse et al. 1974; Tucker 1979)) time series derived from Landsat5/TM and Landsat-7/ETM+ data.

\subsection{Method description}

\subsubsection{Space-time approach for deforestation detection-the concept}

Methods for detecting deforestation events at sub-annual scales (e.g., monthly) from satellite image time series first model the normal forest cover dynamics before assessing deforestation. Modelling normal forest cover dynamics is challenging for pixels which do not have many historical observations. We can remedy this challenge of insufficient historical observations in individual pixel time series by adding the spatial context of the focal pixel in a so-called neighbouring pixels space-time data cube. To do this, we implement the following steps (Figure 3.1). First, like in other studies including (Devries, Verbesselt, et al. 2015; Dutrieux et al. 2015; Hamunyela, Verbesselt \& Herold 2016) we mask non-forest areas in the historical period from the image time series. In this study, we masked non-forest areas using Landsat tree cover continuous fields (Sexton et al. 2013). Second, we define a local space-time data cube (e.g., Figure 3.2) around each pixel in the image time series. Essentially, we have a spatially moving window with an additional time dimension that moves from one pixel to the other. To avoid the smoothing out effect when deseasonalising the data [5], the spatial extent of the data cube should be larger than the size of deforestation events the user aims to detect. Third, we deseasonalise the observations in the local data cube (e.g., Figure 3.3) to increase the sensitivity for detecting non-seasonal changes (see Section 3.2.2). Fourth, we split each local data cube into a reference cube (RC) and monitoring cube (MC). The RC contains historical observations-where nonforest pixels have been masked, whereas the MC contains newly acquired observations - not yet assessed for deforestation. Here, we build upon the idea of specifying the history (reference) and monitoring period as employed in a structural change monitoring framework (Chu et al. 1996; Zeileis et al. 2010; Zeileis et al. 2005; Verbesselt et al. 2012). Fifth, based on training data and observations in the RC, we compute a threshold percentile for defining an observation as abnormally low (see Section 3.2.3). Sixth, we assess whether the 
focal pixel in the $\mathrm{MC}$ is below the threshold percentile. If so, the pixel is flagged as deforestation or potential deforestation.

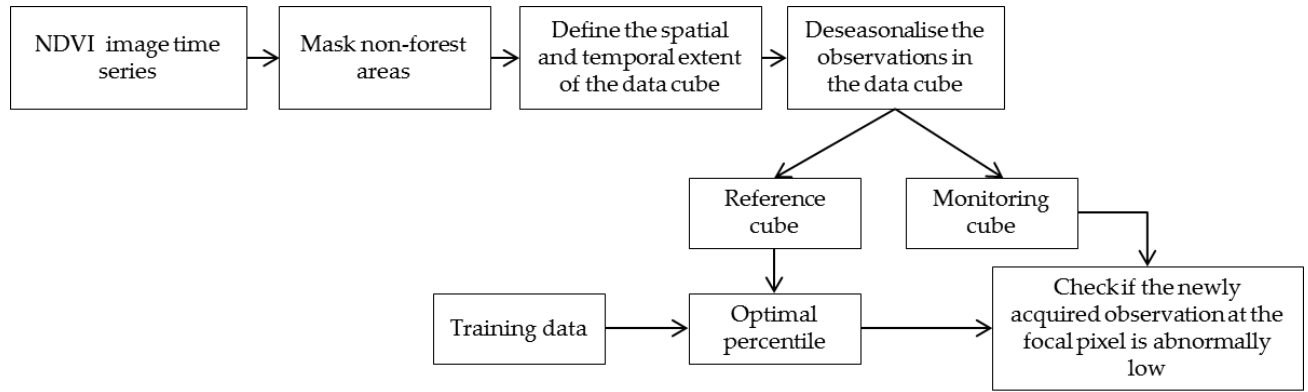

Figure 3.1. The workflow for detecting deforestation at sub-annual as extreme event in space-time data cube.
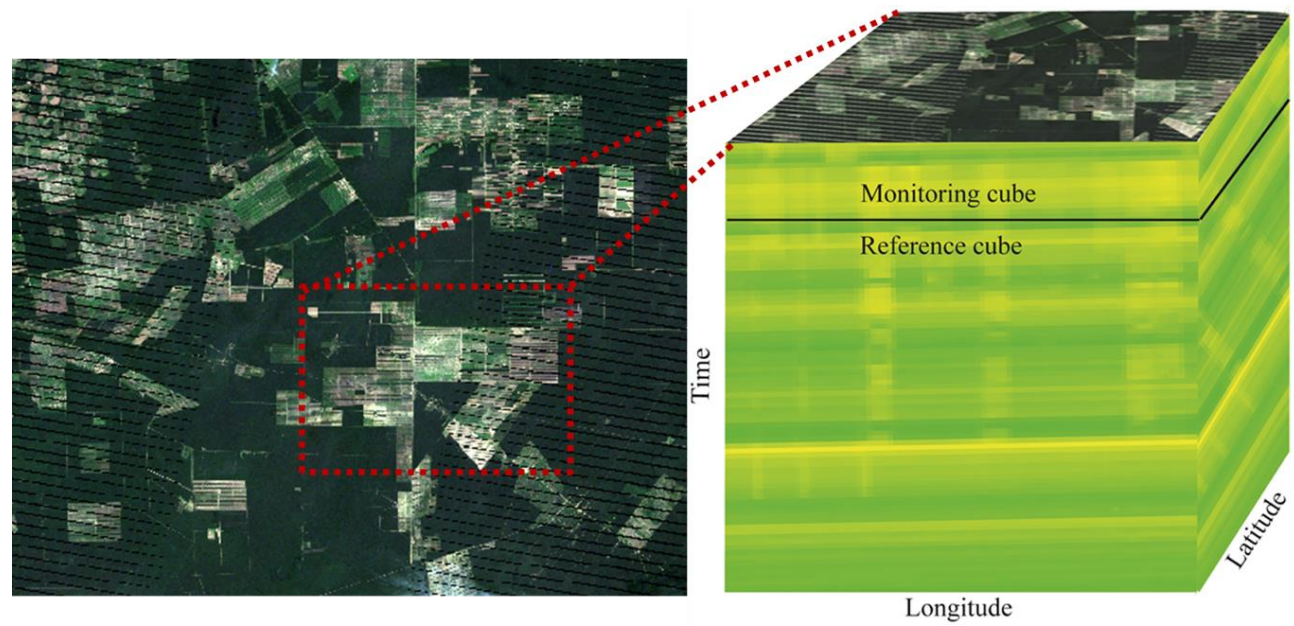

Figure 3.2. An example of a local space-time data cube (right) for normalised difference vegetation index (NDVI) derived from a stack of Landsat images for the period of 2004-2014. The data cube moves across the image stack (moving window), and it is split into a reference and monitoring cube. The NDVI values in the cube are not yet deseasonalised. The horizontal banding in the cube shows the effect of seasonal variations, with yellowish tones representing the dry season. 

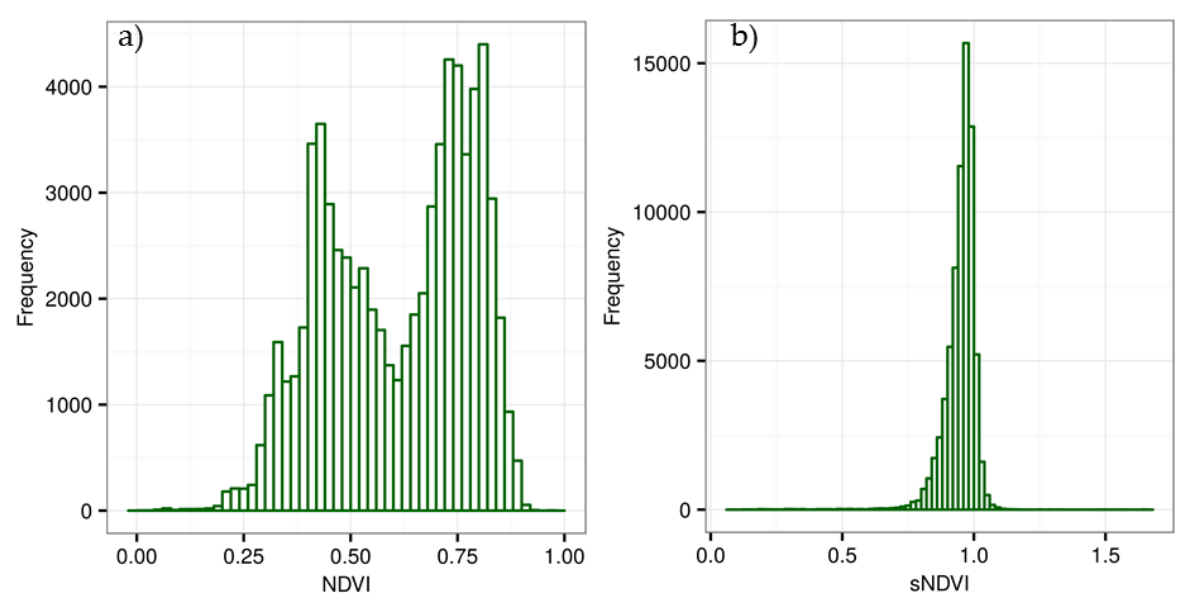

Figure 3.3. An example of distributions of observations in the reference cube before (a) and after (b) reducing the seasonality in a space-time data cube using spatial context. sNDVI denotes spatially normalised NDVI-after applying the spatial context method. Note that some values for spatially normalised NDVI are above 1 because we used 95 percentile to normalise the pixel values. Such values represent NDVI values which were above 95 percentile.

\subsubsection{Deseasonalising observations in the space-time data cube}

Building on the method of (Hamunyela, Verbesselt \& Herold 2016), NDVI time series are deseasonalised by dividing the NDVI value for each pixel with the $95^{\text {th }}$ percentile $\left(\mathrm{P}_{95}\right)$ computed from the NDVI values of the pixels within a defined spatial neighbourhood of such pixel. The use of the $\mathrm{P}_{95}$ is based on the assumption that, for each spatial neighbourhood, forested pixels would occupy the upper tail of the distribution, which is deemed to be properly represented by $\mathrm{P}_{95}$ (Hamunyela, Verbesselt \& Herold 2016). Note that this assumption may not hold if the forest canopy cover is low, or if there are pixels with high vegetation index (VI) values owing to land cover types other than forests (e.g., agriculture). A good forest mask is needed to make sure seasonality for forest pixels can be captured by $\mathrm{P}_{95}$. In contrast to (Hamunyela, Verbesselt \& Herold 2016), where $\mathrm{P}_{95}$ was computed for each pixel, here we compute $\mathrm{P}_{95}$ at each time step in the local data cube, and use it to deseasonalise all pixels per time slice in the data cube. The subscript $t$ in $\mathrm{P}_{95 t}$ denotes a particular time instance t. Within the data cube, we calculate $\mathrm{P}_{95 t}$ for each time instance, and deseasonalise all pixel values at such time instances by dividing them by $\mathrm{P}_{95 t}$. An example of how observations in the reference cube are distributed (a) before and (b) after reducing the seasonality in a space-time data cube using spatial context is shown in Figure 3.3. After deseasonalising, the next step (Section 
3.2.3) is to determine whether a newly acquired observation at the focal pixel in the monitoring period is abnormally low.

\subsubsection{Detecting deforestation as extreme event in space-time data cube}

We rely on the existing extreme value approach (Zscheischler et al. 2013) to compute the threshold from RC for determining if an observation in the monitoring period is abnormally low. With the extreme value approach, observations below a specified percentile (e.g., $5^{\text {th }}$ percentile) are regarded as abnormally low (Zscheischler et al. 2013). Here, a suitable percentile is established by using a training dataset (see Section 3.3.2).

For each local cube, an observation is abnormally low if it is below the specified percentile, and such an observation is initially flagged as potential deforestation. Definite labelling is postponed since satellite image time series are often noisy, mainly because of remnants of clouds and cloud shadows. Furthermore, some pixels may have naturally low values if they cover forest areas that have lower photosynthetic activity. Values for such pixels might be identified as extreme observations within a local space-time data cube, although no deforestation has occurred. If not accounted for, these pixels could lead to the detection of false deforestation events.

Deforestation is confirmed if the next observation is also below the specified percentile regardless of the size of the time step between the observations. Given this monitoring approach, deforestation events are detected with a delay of one observation. This delay is referred to as temporal detection delay, which is the number of observations between when the deforestation event occurred and when the deforestation is detected (Hamunyela, Verbesselt \& Herold 2016). We only assess a pixel for deforestation if such a pixel contains a minimum of three observations, one of which must be in the RC.

\subsection{Monitoring deforestation in Landsat NDVI data cubes-a case study}

We assessed our space-time change detection method at two study sites, shown in Figure 3.4. One study site is a dry tropical forest located southeast of Santa Cruz de la Sierra in Bolivia, (centred at $18.388^{\circ} \mathrm{S}, 62.361^{\circ} \mathrm{W}$ ), and the other study site is a humid tropical forest located west of Ariquemes, Rondonia State, Brazil (centred at $10.2952^{\circ} \mathrm{S}, 64.0478^{\circ} \mathrm{W}$ ). The forest at the Bolivian site is characterised by strong seasonality in its photosynthetic activity, whereas the seasonality is less pronounced at the Brazilian site. Each of the study sites covers an area of about $10,000 \mathrm{~km}^{2}$. Deforestation at the Bolivian site is dominated mainly by industrial agricultural expansion that resulted in large blocks of deforestation events, whereas deforestation events at the Brazilian site are heterogeneous in size, corresponding mostly to a process of 
colonisation. With varying degrees of seasonality and different deforestation processes, these study sites are particularly suitable for testing a new method for sub-annual deforestation monitoring.

We used the NDVI image time series derived from atmospherically (Masek et al. 2006) and geometrically corrected Landsat-5/TM and Landsat7/ETM+ images. Landsat images were obtained from the United State of America's Geological Survey (USGS) Landsat Surface Reflectance (SR) Climate Records (CDR). We used all available (2000-2014) terrain corrected images (L1T). We assumed that co-registration of Landsat-5/TM and Landsat-7/ETM+ images was satisfactory. Clouds and cloud shadows were masked using the Fmask procedure (Zhu \& Woodcock 2012). Note that Fmask outputs are distributed with Landsat SR CDR Products. Landsat tree cover continuous fields for 2005 (Sexton et al. 2013) were used to mask non-forest areas and areas with less than $10 \%$ tree cover prior to the year 2005 .

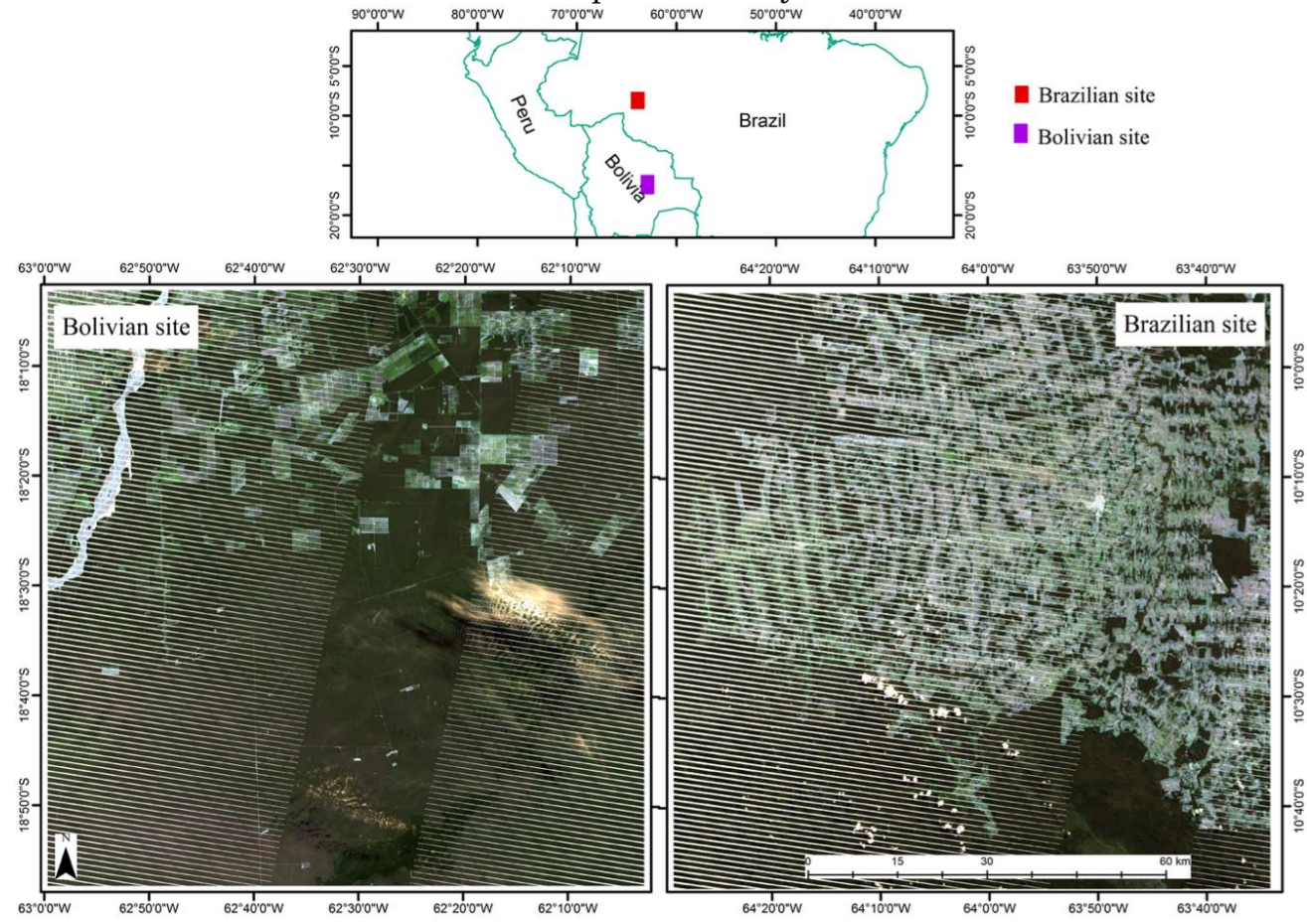

Figure 3.4. An overview of the location of the study sites south east of Santa Cruz de la Sierra, Bolivia, and west of Ariquemes, Rondonia State, Brazil. The base images are band 1-2-3 composites of Landsat ETM+ images from 15 April 2013 (Bolivia) and 21 July 2014 (Brazil).

To understand how the spatial extent of the local data cube influences spatial and temporal accuracies, we tested six varying spatial extents of the 
local data cube (Table 3.1). We also varied the temporal extent of the RC, herein referred to as temporal extent of the data cube, to understand how it affects spatial and temporal accuracy for deforestation detection. The temporal extent was varied from one to five years of data, at an interval of one year. The $\mathrm{RC}$ contained images from 2004 for the one-year data scenario, images from 2003-2004 for the two-year scenario, and images from 2002-2004 for the three-year data scenario. For the four- and five-year data scenarios, the RC contained images from 2001-2004 and 2000-2004, respectively. Figure 3.5 shows the number of images available in the RC for each temporal extent at each study site. For each spatial and temporal extent, we trained our method to determine the optimal percentile for detecting deforestation at a sub-annual scale (Section 3.3.2). Next, we used the optimal percentiles to validate our method (Section 3.3.3).

Table 3.1. Data cube names and their spatial extents and dimensions for the Bolivian and Brazilian sites.

\begin{tabular}{ccc}
\hline $\begin{array}{c}\text { Name of the Data } \\
\text { Cube }\end{array}$ & $\begin{array}{c}\text { Spatial Dimensions the Data } \\
\text { Cube } \\
\text { (Row } \times \text { Column) }\end{array}$ & $\begin{array}{c}\text { Spatial Extent of the Data } \\
\text { Cube } \\
\text { (ha) }\end{array}$ \\
\hline C5 & $5 \times 5$ & 2.25 \\
C9 & $9 \times 9$ & 7.29 \\
C13 & $13 \times 13$ & 15.21 \\
C17 & $17 \times 17$ & 26.01 \\
C21 & $21 \times 21$ & 39.69 \\
C25 & $25 \times 25$ & 56.25 \\
\hline
\end{tabular}

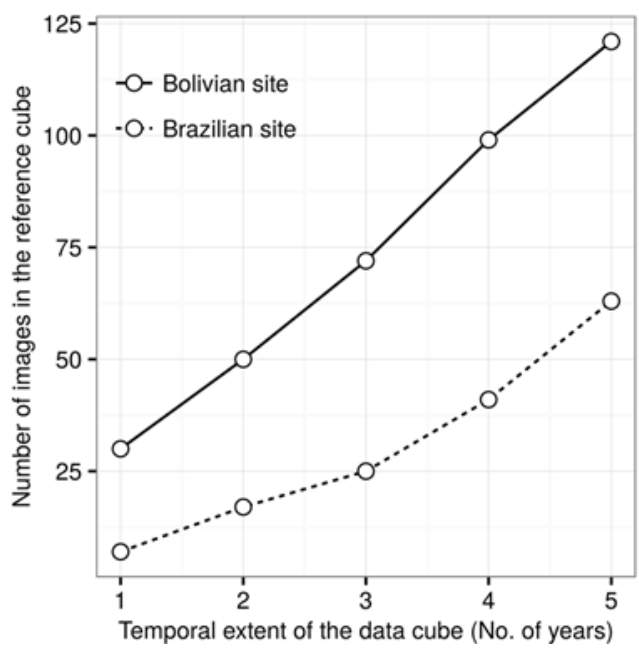

Figure 3.5. Cumulative number of images in the reference cube versus the temporal extent of the reference cube at Bolivian and Brazilian study sites. 


\subsubsection{Reference data}

We used 966 sample pixels to validate the final change map for the Bolivian site, and 400 sample pixels were used for validating the Brazilian change map (Table 3.2). Training was based on 170 and 70 sample pixels for the sites in Bolivia and Brazil, respectively. Similar to (Cohen et al. 2010; Dutrieux et al. 2015; Devries, Verbesselt, et al. 2015), reference data were acquired by visual interpretation of Landsat data along with high spatial resolution imagery available in Google Earth and Bing Maps . High spatial resolution imagery available in Google Earth and Bing Maps were used to determine whether an area is indeed deforested or not. At each study site, we sampled deforested and forested areas by stratified probability sampling (Stehman 2009; Olofsson et al. 2014) after manually digitising corresponding areas on Landsat images. The number of sample pixels was proportional to the area of the stratum. The deforested stratum contained areas that had been deforested during the period of 2005-2014, whereas the forested stratum covered areas which were still forested at the end of 2014. For each sample pixel in the deforested area, we estimated the date of deforestation by visually determining the Landsat image in which the deforestation event is first visible. The date of deforestation was used to assess the temporal accuracy.

Table 3.2. Number of sample pixels used for training purposes and final validation of deforestation maps produced at the Bolivian and Brazilian sites.

\begin{tabular}{lcccc}
\hline & \multicolumn{2}{c}{ Bolivian Site } & \multicolumn{2}{c}{ Brazilian Site } \\
\cline { 2 - 5 } & Forest & Deforested & Forest & Deforested \\
\hline Training data & 155 & 15 & 49 & 21 \\
Test data & 878 & 88 & 280 & 120 \\
\hline
\end{tabular}

\subsubsection{Training the space-time change detection method}

To train our method, we generated a series of percentiles $(n=50)$, ranging from 0.1 to the $5^{\text {th }}$ percentile, at an interval of 0.1 percent. Next, for each spatial and temporal extent, we used each of these percentiles as a threshold for identifying deforestation at a sub-annual scale. A training data set (Table 3.2) was then used to calculate the overall accuracy, bias (calculated by subtracting the omission error from the commission error (Reiche 2015)), and the median temporal detection delay. Since our monitoring goal was to detect deforestation events as early as possible but with high overall accuracy, for each spatial and temporal extent we selected the percentile, i.e., the optimal percentile, that achieved the shortest median temporal detection delay with the highest overall accuracy. 


\subsubsection{Validating the space-time change detection method}

At each study site, we applied our space-time change detection method using the optimal percentiles, determined from the training data, as thresholds for deforestation. We emulated a "near real-time" monitoring scenario, implying that observations in the monitoring period were sequentially rather than simultaneously assessed for deforestation. Although some areas may have experienced multiple deforestation and regrowth regimes between 2005 and 2014, we only considered the first deforestation event per pixel, and once labelled as deforested, we stopped monitoring such a pixel at subsequent time steps. The spatial and temporal accuracy for change detected between 2005 and 2014 were calculated using the test data set (Table 3.2). More specifically, we calculated the overall accuracy, producer's accuracy, user's accuracy, and the median temporal detection delay. Like (Hamunyela, Verbesselt \& Herold 2016), the temporal detection delay was calculated at each sample point by counting the number of valid observations available between the image in which deforestation was visually identified, and the image in which deforestation was detected by our method. Confidence intervals for the overall accuracy, as well as producer's and user's accuracies, were calculated using binomial probability of success based on Wilson's method (Wilson 1927)

\subsection{Results}

In this section, first we show the results from the training phase of our method (Section 3.4.1). In particular, we show how the percentile threshold affects the spatial and temporal accuracy (Section 3.4.1.1), and how spatial and temporal extents of the data cube influence the optimal percentile for detecting deforestation at a sub-annual scale. Next, we show the validation results (Section 3.4.2).

\subsubsection{Training}

\subsubsection{Effect of percentile threshold on spatial and temporal accuracy}

Figure 3.6 shows an example of how overall accuracy, bias, and median temporal detection delay were changing in relation to the percentile threshold. Here, we only show examples for the smallest (C5) and largest (C25) cubes for each study site. Generally, larger percentiles produced lower overall accuracy (Figure 3.6a,d) and shorter median temporal detection delay (Figure 6c,f), except for the C25 at Brazilian site. Deforestation events were increasingly being omitted (negative bias) when using smaller percentiles (Figure 3.6b,e). 


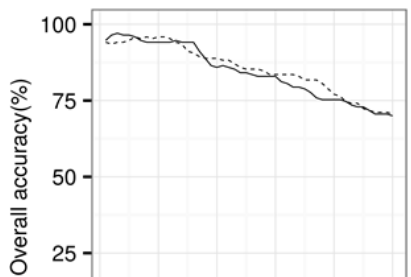

a)

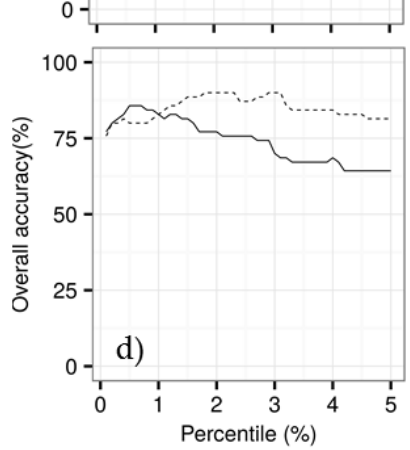

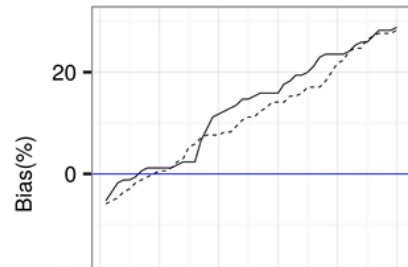

b)

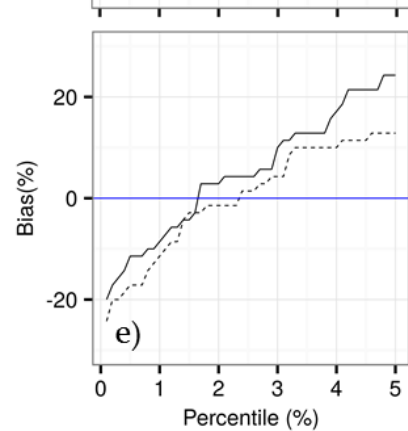

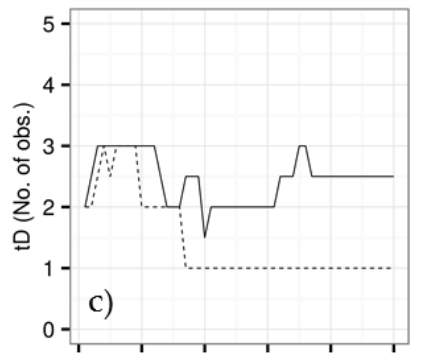

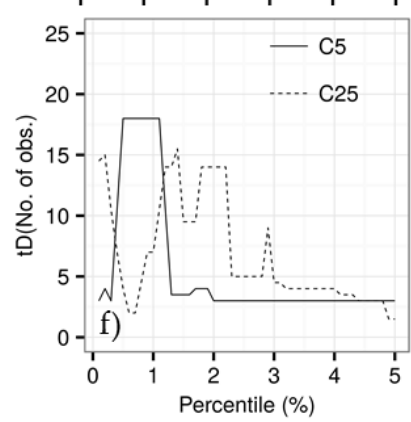

Figure 3.6. Change in the overall accuracy, bias, and the median temporal detection delay (tD) as a function of the percentile for defining deforestation as extreme event in Landsat space-time data cubes (C5 and C25) at the Bolivian $(\mathrm{a}-\mathrm{c})$ and Brazilian $(\mathrm{d}-\mathrm{f})$ study sites. The blue lines denote zero bias. Bias was calculated by subtracting the omission error from the commission error (Reiche 2015).

\subsubsection{Effect of spatial and temporal extent of the data cube on optimal percentile}

Figure 3.7 shows how the optimal percentile changes for different combinations of spatial and temporal extents. For longer temporal extents (TE $=4$ or 5 years), the optimal percentile is higher for larger spatial extents (Figure 3.7). Generally, the optimal percentiles at the Brazilian site were larger than those for the Bolivian site. At the Bolivian site, the optimal percentile was smaller than $3 \%$ for each spatial and temporal extent combination. 


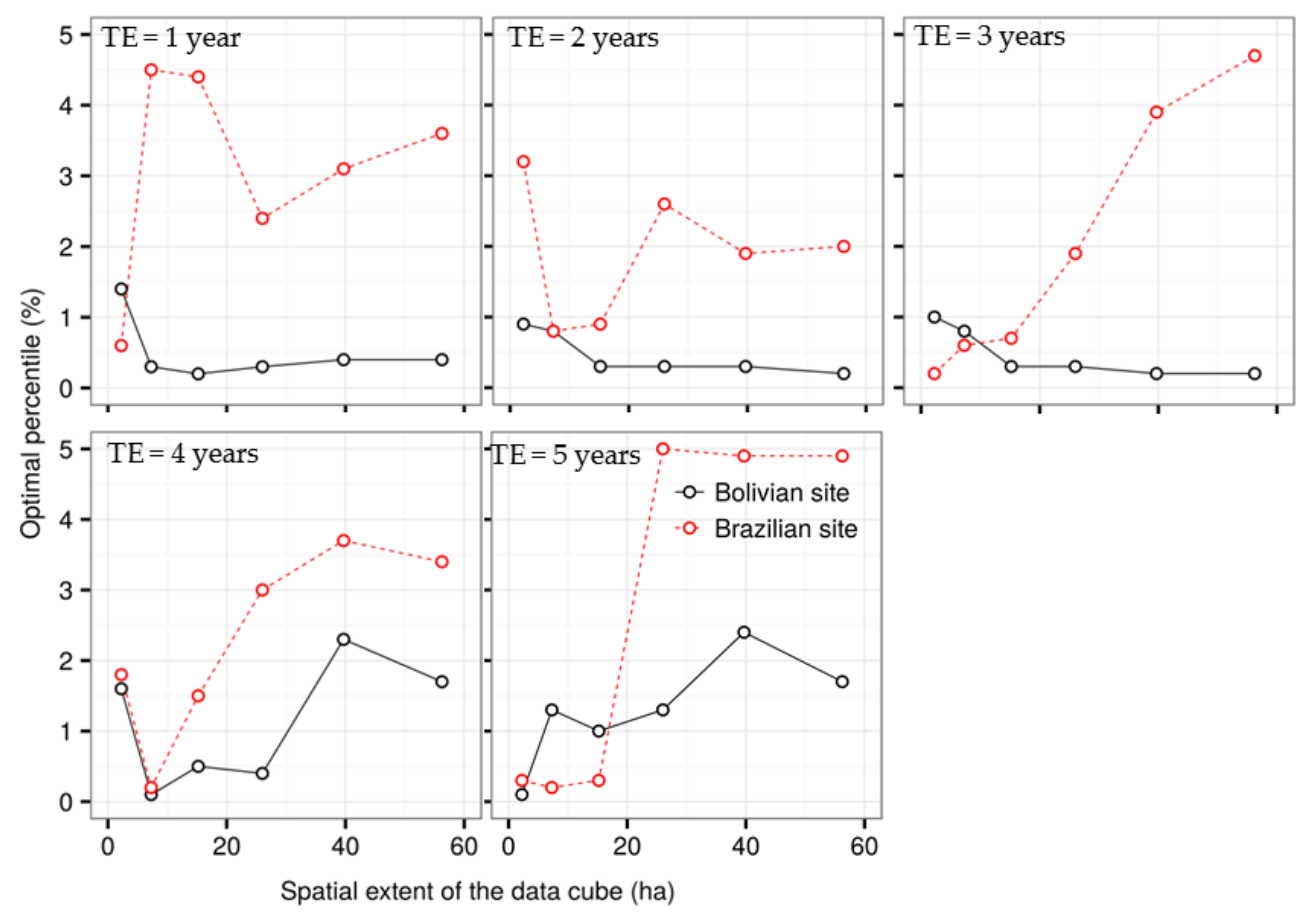

Figure 3.7. Change in the optimal percentile for detecting deforestation events at sub-annual scales when varying the spatial extent and temporal extent (TE) of the data cube at the Bolivian and Brazilian site. The optimal percentile was determined by choosing the percentile that achieves the shortest median temporal detection delay and highest overall accuracy based on the training data set.

\subsubsection{Validation}

Figure 3.8 shows how the spatial and temporal accuracies varied in relation to spatial and temporal extents. Note that the effect of the spatial extent on spatial and temporal accuracy (Figure 3.8a,c,e,g), is shown for the results obtained from the longest temporal extent ( $\mathrm{TE}=5$ years). The effect of the temporal extent is shown for the results obtained from the largest spatial extent (56.25 ha, Figure $3.8 \mathrm{~b}, \mathrm{~d}, \mathrm{f}, \mathrm{h})$. Varying the spatial extent or temporal extent had a marginal influence on the overall accuracy at both the Bolivian and the Brazilian site (Figure 3.8a,b). However, the change in either the spatial extent or temporal extent of the data cube had a pronounced effect on the producer's (Figure $3.8 \mathrm{c}, \mathrm{d}$, ) and user's accuracies (Figure 3.8e,f). Increasing the spatial or temporal extent of the data cube resulted in higher producer's accuracy and lower user's accuracy at both sites. Data cubes with a smaller spatial extent (e.g., smaller 
than 20 ha) produced maps with a higher user's accuracy at both study sites than larger data cubes. The reference cube with the shortest temporal extent (one year of data) produced maps with $74.4 \%$ user's accuracy at the Bolivian site, and $65.8 \%$ at the Brazilian site. Deforestation events detected at Bolivian and Brazilian sites when the reference cube contained one year of data are shown by month (Figure 3.9) and year (Figure 3.10) of detection. The variation in producer's accuracy was generally larger when varying the spatial extent of the cube than when varying the temporal extent (Figure 3.8c,d).

Increasing the spatial or temporal extent of the data cube resulted in a shorter median temporal detection delay at both study sites (Figure $3.8 \mathrm{~g}, \mathrm{~h}$ ). However, the change in temporal extent did not have an influence on the median temporal detection delay at the Brazilian site. At this site, reference cubes with a temporal extent of four or five years achieved the shortest median temporal detection delay (one observation). Similar to the producer's accuracy, the variation in median temporal detection delay was generally greater when varying the spatial extent of the cube than when varying the temporal extent (Figure $3.8 \mathrm{~g}, \mathrm{~h}$ ).

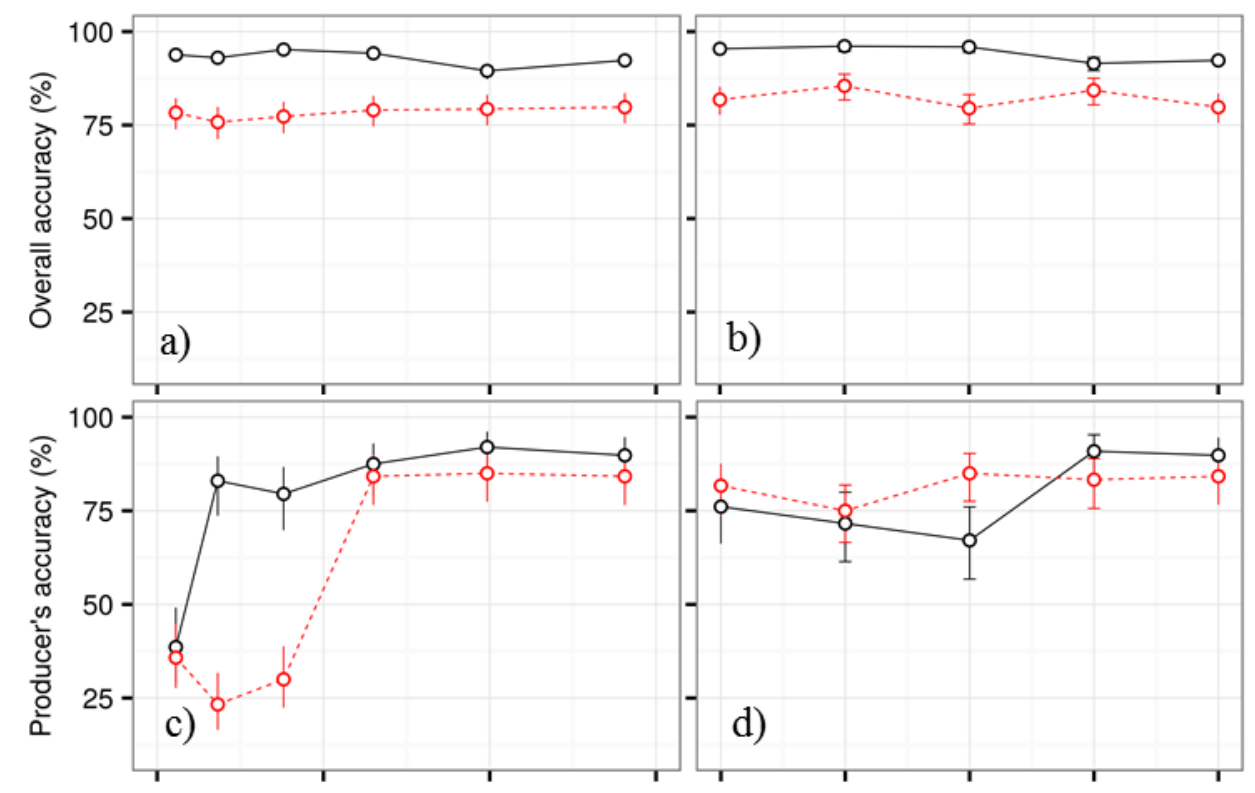




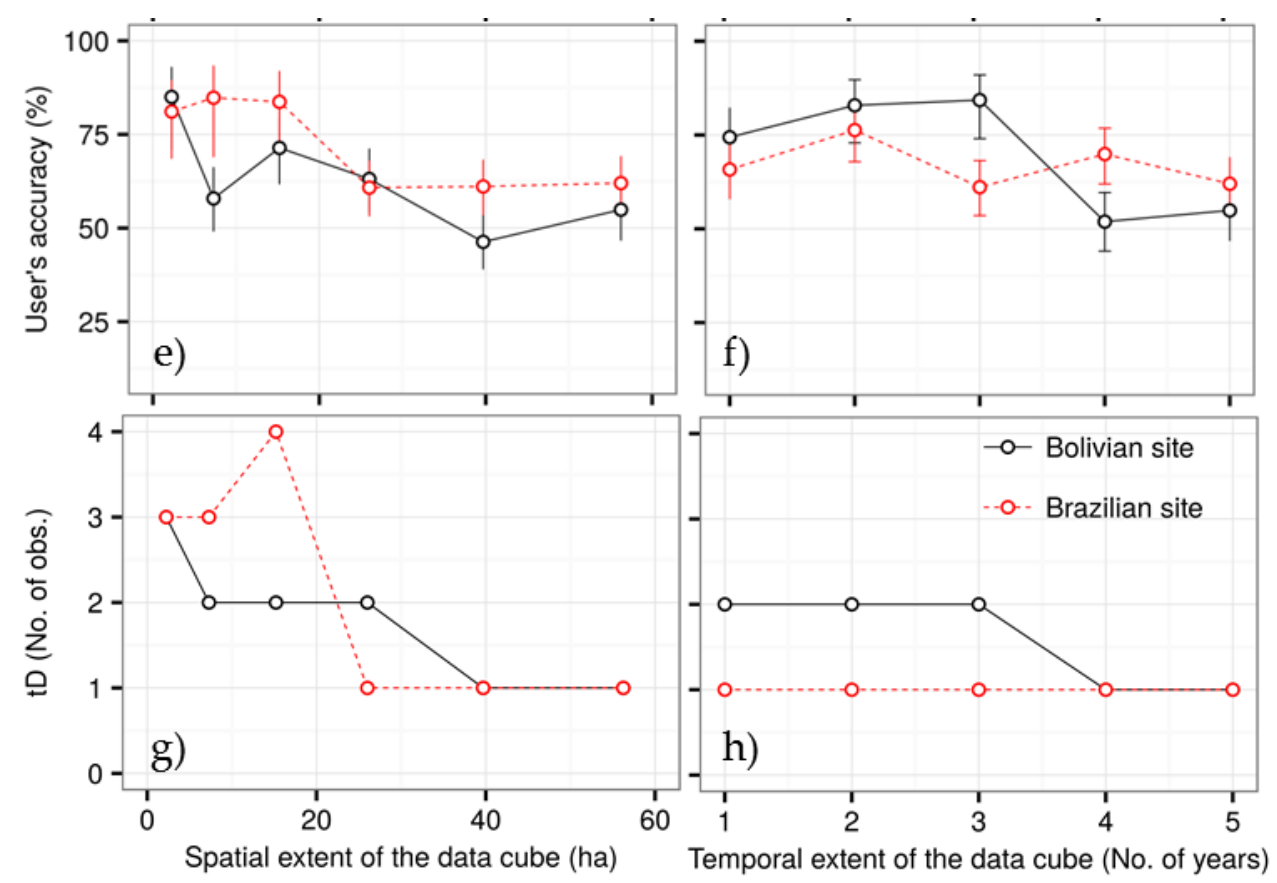

Figure 3.8. Overall accuracy (a,b), producer's accuracy (c,d), user's accuracy $(\mathrm{e}, \mathrm{f})$, and median temporal detection delay $(\mathrm{tD})(\mathrm{g}, \mathrm{h})$ of deforestation events at the Bolivian and Brazilian study sites as a function of the spatial (left) and temporal extent (right) of the local data cube. Vertical bars (a-f) indicate $95 \%$ confidence intervals. The effect of the spatial extent on spatial and temporal accuracy (Figure 3.8a,c,e,g), is shown for the results obtained from the longest temporal extent $(\mathrm{TE}=5$ years). The effect of the temporal extent is shown for the results obtained from the largest spatial extent (56.25 ha, Figure 3.8b,d,f,h). 

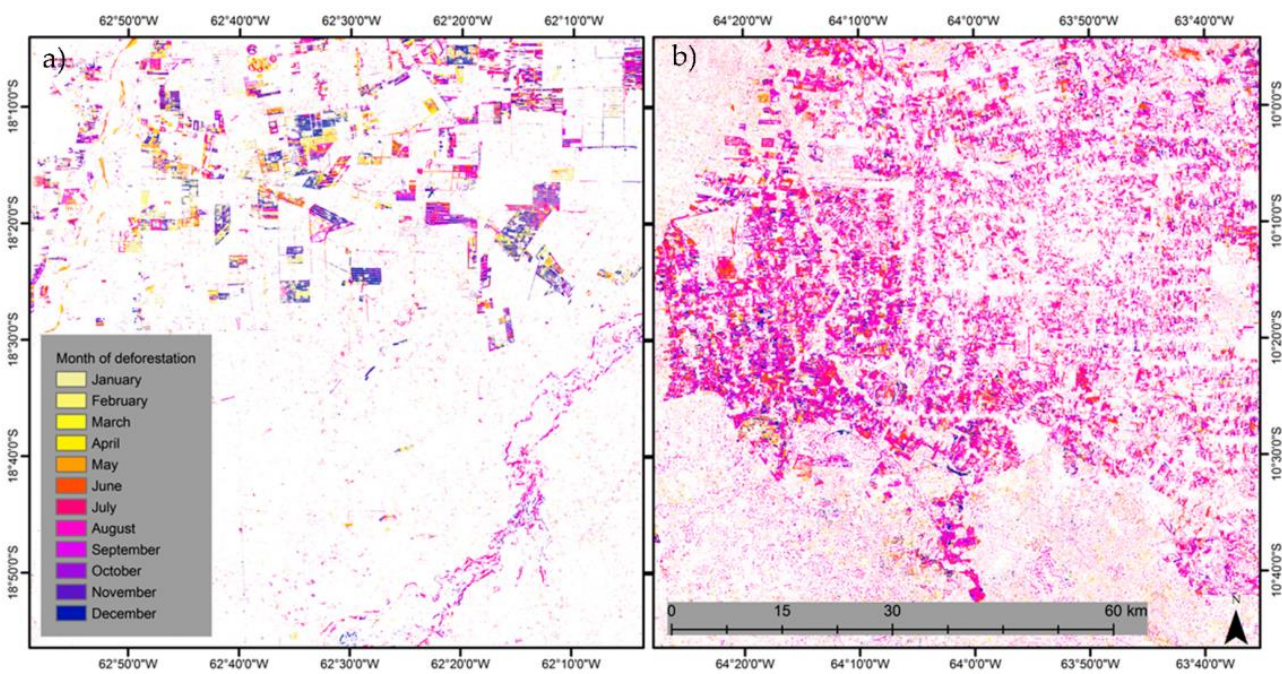

Figure 3.9. The month in which deforestation events detected from Landsat time series at Bolivian site (a) and Brazilian site (b) over the period of 20052014 occurred. Deforestation was detected as an extreme event in Landsat data cube with a reference period containing one year of data.
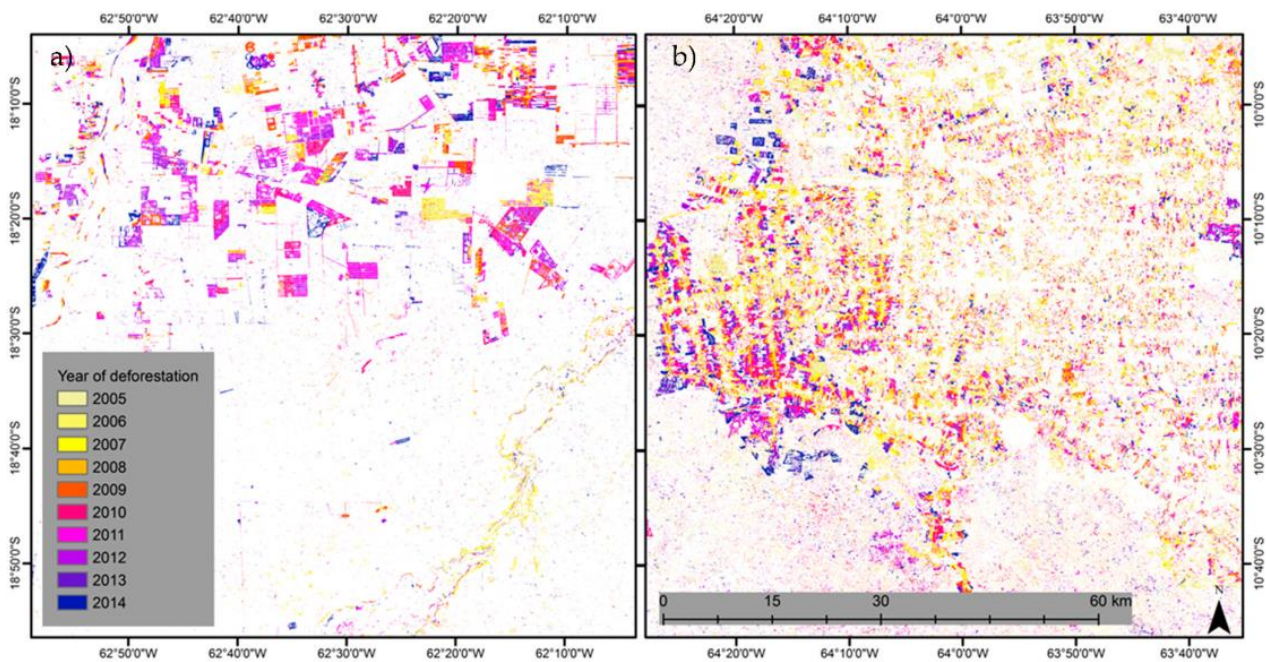

Figure 3.10. The year in which deforestation events detected from Landsat time series at Bolivian site (a) and Brazilian site (b) over the period of 20052014 occurred. Deforestation was detected as an extreme event in Landsat data cube with a reference period containing one-year of data. 


\subsection{Discussion}

In this chapter, we proposed a data-driven space-time change detection method that exploits spatiotemporal information in satellite image time series to detect deforestation at sub-annual scales as extreme events in local data cubes. We demonstrated the space-time change detection method on Landsat NDVI image time series at a dry (Bolivian site) and humid (Brazilian site) tropical forest sites. Our results show that the method is suitable for accurate detection of deforestation events at sub-annual scales in both dry and humid forests even when image time series contains only one year of historical observations. We were able to achieve a median temporal detection delay of less than three observations, and producer's accuracy above $70 \%$, user's accuracy above $65 \%$, and an overall accuracy above $80 \%$ at both dry and humid tropical forest areas when using a data cube with one year of historical observations and a window size of 56.25 ha (Figure 3.8). A previous study at the same study sites (Hamunyela, Verbesselt \& Herold 2016), which used a method that only analysed individual pixel time series (Verbesselt et al. 2012)and used all available Landsat images (1984-2014), also achieved at overall accuracy above $80 \%$ and a median temporal detection delay of less than four observations. Other studies (Zhu \& Woodcock 2014b; Dutrieux et al. 2015; Reiche, de Bruin, et al. 2015) which used different methods to detect deforestation at sub-annual scales at different study sites, also achieved overall accuracies above $80 \%$. These studies expressed the temporal delay in time, whereas here we express the temporal detection delay as number of observations, thus making it difficult to directly compare their temporal delay to ours.

Deforestation events were mapped more accurately at the Bolivian site when using data cubes with a large spatial extent. This is mainly because deforestation events at the Bolivian site were generally large. In areas with large deforestation events, cubes with a larger spatial extent lead to accurate deforestation mapping because the cube is less likely to be entirely within the footprint of a deforestation event. If the spatial extent of a data cube is smaller than the footprint of the deforestation event, the impact of deforestation is likely to be smoothed out when spatially normalising data to reduce seasonal variations. The spatial normalisation approach assumes that there are at least $5 \%$ forest pixels within the spatial window, since pixels' values are spatially normalised against the upper 5\% tail (Hamunyela, Verbesselt \& Herold 2016). Using data cubes with small spatial extent can lead to accurate mapping of deforestation events in areas with relatively small deforestation events. With small deforestation events, spatial normalisation is less likely to smooth out the impact of deforestation in the data. This is why deforestation events were mapped accurately at the Brazilian site even when using data cubes with small spatial extent. 
The incidents of false detection (low user accuracy) were particularly high for data cubes with large spatial extents because the thresholds calculated from data cubes with large spatial extents or longer temporal extents were relatively large. Such large thresholds can lead to many false detections because they might be too sensitive. Such sensitivity could also explain why deforestation events were typically detected with shorter delay when using data cubes with either large spatial extent or longer temporal extent (Figure 3.8). Sensitive thresholds can also explain why increasing the temporal extent of the data cube at the Bolivian site led to accurate mapping of deforestation events. Increasing the temporal extent of the data cube did not affect the temporal detection delay at the Brazilian site (Figure $3.8 \mathrm{~h}$ ). This is mainly because, for each temporal extent, the optimal percentile at the Brazilian site was large (Figure 3.7).

Increasing the spatial extent had more of a major influence on the optimal percentile at the Brazilian site than at the Bolivian site (Figure 3.7). This inter-site difference can be explained by the number of observations in the reference cube. Temporally, there were fewer images at the Brazilian site than at the Bolivian site (Figure 3.5). So, optimal percentiles at the Brazilian site were less likely to reach stability at smaller spatial extents because observations in the reference cube were few. In contrast, at the Bolivian site, the optimal percentile was most likely to reach stability at smaller spatial extents because the images were many, and additional information from spatial context was less likely to have a major influence on the optimal percent.

Our method offers new opportunities to tackle challenges associated with existing methods for monitoring deforestation at sub-annual scales (Verbesselt et al. 2012; Dutrieux et al. 2015; Reiche, de Bruin, et al. 2015; Hamunyela, Verbesselt \& Herold 2016; Zhu et al. 2012). In particular, our method exploits both spatial and temporal information in satellite image time series to detect deforestation at a sub-annual scale, thus allowing us to analyse pixels which do not have many historical observations. Results from the two case studies indicate that our method is robust in detecting deforestation events at a subannual scale, even when the image time series only contains one year of historical observations (Figure 3.8). One year of historical observations is often too short to properly differentiate deforestation from normal forest dynamics, especially in forests that exhibit strong seasonality. By combining spatial and temporal information, we can use image time series of high spatial resolution satellite sensors (e.g., RapidEye), whose time series are short, to track smallscale forest disturbances (e.g., selective logging). Similarly, by exploiting spatiotemporal information in image time series, there is no need to wait for image time series from newly launched sensors (e.g., Sentinel-2) to lengthen before exploiting such data to detect deforestation events at sub-annual scales. Since our method remains robust in detecting deforestation at sub-annual 
scales even when the reference period only contains one year of data, users may not need to pre-process huge amounts of historical data when monitoring deforestation at sub-annual scales.

The method presented in this chapter can be applied in different forest areas because it is a data-driven approach using thresholds computed from the data. However, it may face challenges in mixed forests, where deciduous and evergreen forests coexist at short distances. This is mainly because of the way we reduce seasonal variations in the data cube. Normalisation against $P_{95 t}$ (Section 3.2.2) is not likely to reduce seasonal variations because $\mathrm{P}_{95 t}$ would represent evergreen trees in mixed stands. This limitation may be addressed by calculating $\mathrm{P}_{95 t}$ for each forest type separately and by deseasonalising pixels from each forest type using the corresponding P95t. Another limitation is related to how we treat the pixels whose historical observations also qualify as extremes although no deforestation has occurred.

We determined optimal percentiles for identifying deforestation at subannual scale, but these percentiles might not be optimal for other areas with different forest types and processes causing deforestation. In areas with gradual changes (Devries, Verbesselt, et al. 2015) for example, smaller percentiles ( $5^{\text {th }}$ percentile) might be preferable. Therefore, users should calibrate the method to identify optimal percentiles for their respective study areas before monitoring for deforestation at sub-annual scales. To do this, users should select sample pixels from both deforested and forested areas in their study areas, and apply space-time change detection method while varying the percentile for deforestation detection (e.g., in Section 3.3.2). Depending on the monitoring goal, for example a shorter temporal detection delay, the user can then selects the percentile that achieves the shortest median temporal delay as the optimal percentile.

We tested several window sizes (spatial extents) of the data cube, but identifying a window size appropriate for different parts of the globe is still challenging. This is because a window size which is optimal in one area might not be optimal in another area. Prior knowledge on the size of the deforestation events that typically occur in a particular area can be used to decide on the spatial extent of the data cube. If such prior knowledge is lacking, the user should choose a spatial extent which is larger than the size of deforestation events the user aims to detect.

With the advent of open and free access to data from Sentinel sensors, especially Sentinel-1 and -2, detecting deforestation at small spatial scales within few days of occurrence will be possible. Combining Sentinel and Landsat data will boost monitoring of deforestation at sub-annual scales, allowing agencies responsible for forest protection to timely intervene in areas where illegal deforestation events are occurring. However, such multi-source data would need harmonisation to produce multi-sensor time series which is temporally consistent. 


\subsection{Conclusion}

In this chapter, we demonstrated how spatial and temporal information can be combined and exploited to detect deforestation from satellite image time series at a sub-annual scale. We proposed a data-driven space-time change detection method that detects deforestation as an extreme event within a space-time data cube of satellite image time series. We detected sub-annual deforestation from Landsat NDVI time series at a dry tropical forest site, where the forest exhibits strong seasonality, and at humid tropical forest site. The method remained robust in detecting deforestation events at a sub-annual scale even when the image time series only contained one year of historical observations. The space-time method we presented in this chapter is a novel and robust approach for timely detection of deforestation events in areas where forests exhibit strong seasonality. It provides an opportunity to detect deforestation events using image time series with scarce historical observations. The method can be used in different types of forest, both evergreen and deciduous, but, it may face a challenge in mixed forests, where deciduous and evergreen forests coexist at short distances. Although we used NDVI, the method is expected to be applicable for image time series of any satellite-derived metric that is used for deforestation monitoring. To further improve deforestation monitoring at a sub-annual scale, future research should investigate how data from different satellite sensors (i.e., Landsat 7 and 8, Sentinel-2, RapidEye, and SPOT) can be combined in a space-time change detection framework to facilitate near real-time deforestation detection.

\section{Acknowledgements}

This work was funded through Google Deforestation Monitoring project (grant agreement 5120906), the European Union Seventh Framework Programme (FP7/2007-2013; grant agreement 283093)-The Role of Biodiversity in Climate Change Mitigation (ROBIN), and European Space Agency (ESA) ForMoSa-Forest Degradation Monitoring with Satellite Data Project (grant agreement 5160957022). 

Chapter

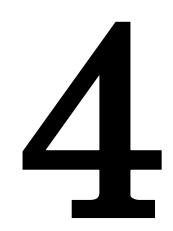

\section{Using space-time features to improve detection of forest disturbances from Landsat time series}

This chapter is based on:

Hamunyela, E., Reiche, J., Verbesselt, J., \& Herold, M. Using space-time features to improve detection of forest disturbances from Landsat time series. Remote Sensing, 9, 515. 


\begin{abstract}
Current research on forest change monitoring using medium spatial resolution Landsat satellite data aims for accurate and timely detection of forest disturbances. However, producing forest disturbance maps that have both high spatial and temporal accuracy is still challenging because of the trade-off between spatial and temporal accuracy. Timely detection of forest disturbance is often accompanied by many false detections, and existing approaches for reducing false detections either compromise the temporal accuracy or amplify the omission error for forest disturbances. Here, we propose to use a set of space-time features to reduce false detections. We first detect potential forest disturbances in the Landsat time series based on two consecutive negative anomalies, and subsequently use space-time features to confirm forest disturbances. A probability threshold is used to discriminate false detections from forest disturbances. We demonstrated this approach in the UNESCO Kafa Biosphere Reserve located in the southwest of Ethiopia by detecting forest disturbances between 2014 and 2016. Our results show that false detections are reduced significantly without compromising temporal accuracy. The user's accuracy was at least $26 \%$ higher than the user's accuracies obtained when using only temporal information (e.g. two consecutive negative anomalies) to confirm forest disturbances. We found the space-time features related to change in spatio-temporal variability, and spatio-temporal association with non-forest areas, to be the main predictors for forest disturbance. The magnitude of change and two consecutive negative anomalies, which are widely used to distinguish real changes from false detections, were not the main predictors for forest disturbance. Overall, our findings indicate that using a set of space-time features to confirm forest disturbances increases the capacity to reject many false detections, without compromising the temporal accuracy.
\end{abstract}

Keywords: Space-time features, Data cubes, Landsat, Change detection, Forest disturbance 


\subsection{Introduction}

Current research on forest change monitoring aims for accurate and timely detection of forest disturbances. Many change detection approaches have therefore been proposed in recent years to improve the detection of forest disturbances from medium spatial resolution satellite data (e.g. Landsat) (Zhu et al. 2012; Xin et al. 2013; Zhu \& Woodcock 2014b; Hilker, Wulder, Coops, Seitz, et al. 2009; Verbesselt et al. 2012; Hamunyela, Verbesselt, Bruin, et al. 2016; Reiche, de Bruin, et al. 2015; Hansen et al. 2016). The proposed approaches have addressed many key challenges, including seasonality in dry forests (Dutrieux et al. 2015; Hamunyela, Verbesselt \& Herold 2016; Reiche et al. in review). However, it is still challenging to produce forest disturbance maps which have both high spatial and temporal accuracy. There is always a trade-off between spatial and temporal accuracy (Zhu et al. 2012; Hamunyela, Verbesselt, Bruin, et al. 2016; Reiche, de Bruin, et al. 2015; Reiche et al. in review). A shorter temporal detection delay is typically associated with high producer's accuracy and low user's accuracy (Reiche et al., in review). Low user's accuracy implies that timely detection of forest disturbances is accompanied by many false detections. False detections in satellite time series may occur because of insufficient deseasonalisation or because of the presence of sensors' artefacts and remnant atmospheric contaminations (Vermote et al. 2009; Zhu et al. 2012). Yet, existing approaches lack capacity to reject false detections without compromising the temporal accuracy. In cases where high user's accuracy has been reported, the omission error for small-scale forest disturbances was high (Hansen et al. 2016). In short, amplified false detections and high omission error for small-scale forest disturbances are two major challenges that currently hinder accurate and timely detection of forest disturbances.

Recent studies attempted to remedy the problem of false detections by using a threshold on the magnitude of change, to discriminate real disturbances from false detections (Devries, Verbesselt, et al. 2015; Hamunyela, Verbesselt \& Herold 2016). The use of a threshold on the magnitude of change is based on the assumption that, when compared to real changes, false detections have smaller magnitude of change. This assumption is problematic, however, because forest disturbances that involve partial removal of forest cover may also have small magnitude of change (Huang et al. 2009). Small-scale and gradual forest disturbances, which are widely common especially in Africa, caused mainly by small-holder agriculture expansion, domestic firewood and charcoal extractions (Fisher 2010) also tend to have small magnitude of change (Devries, Verbesselt, et al. 2015). Recent studies show that false detections are particularly common in areas where small-scale forest disturbances, caused by small-holder agriculture and human settlements expansion, are prevalent (Schultz et al. 2016). Separating forest disturbances 
from false detections using a threshold on the magnitude of change in such areas may therefore lead to high omission error for small-scale forest disturbances. The difficulty in separating false detections and forest disturbances may explain why small-scale forest disturbances are still a major constraint to accurate mapping and quantification of forest loss (Tyukavina et al. 2013). It is therefore important that new change detection approaches should be able to improve the detection of small-scale forest disturbances in order to achieve accurate mapping and quantification of forest loss.

Alternative to magnitude of change, some change detection approaches use consecutive anomalies to confirm forest disturbance. Essentially, forest disturbance is only confirmed if there are at least two or more consecutive negative anomalies in the time series (Zhu \& Woodcock 2014b; Hamunyela, Verbesselt, Bruin, et al. 2016; Reiche, de Bruin, et al. 2015; Hansen et al. 2016). An observation is considered as negative anomaly if it is below a specified threshold (Zscheischler et al. 2013). The approach of consecutive anomalies is based on the assumption that the presence of multiple consecutive negative anomalies in the time series may be strong indicator for forest disturbance because the impact of forest disturbances is typically persistent through time (Zhu et al. 2012). Consecutive negative anomalies are also considered more robust against noise in the time series than a threshold on the magnitude of change (Zhu \& Woodcock 2014b; Zhu et al. 2012; Reiche, de Bruin, et al. 2015). To detect forest disturbances timely using a consecutive negative anomaly approach, however, a threshold for defining an observation as an anomaly has to be less strict (Hamunyela, Verbesselt, Bruin, et al. 2016; Reiche et al. in review). Unfortunately, a less strict threshold amplifies false detections (Hamunyela, Verbesselt, Bruin, et al. 2016). In addition to consecutive anomalies, other approaches use temporal metrics derived from pixel-time series of Landsat spectral bands and indices to calculate the likelihood of forest loss (Hansen et al. 2016). Forest loss is confirmed when there are two consecutive observations at the pixel showing a higher likelihood of forest loss (Hansen et al. 2016). This approach is particularly novel because the likelihood of forest loss is calculated based on a set spectral and temporal metrics. Overall, this forest change detection approach produced a forest disturbance map with high user's accuracy in Peru, but the omission error for small-scale forest disturbances was high (Hansen et al. 2016).

Solving the problems of false detections and omission of small-scale forest disturbances remains challenging mainly because existing forest change detection approaches only rely on temporal and spectral information. They ignore spatial information. Yet, forest disturbances, by default, are spatiotemporal processes (Alves 2002). Oftentimes, new disturbances, especially those induced by humans, have both spatial and temporal association to old disturbances (Alves 2002). This spatio-temporal association arises because new loggings, for example, are more likely to occur within the neighbourhood of 
previously logged areas or logging routes than in areas which are far from previous disturbances. Essentially, a set of "space-time features" can be extracted from satellite data cubes and subsequently use such features as indicators for forest disturbance in order to discriminate false detections from real disturbances. To the best of our knowledge, this idea of using space-time features to discriminate false detections from real disturbances has not yet been investigated.

The objective of this chapter was to investigate whether the detection of forest disturbances from Landsat time series can be improved by using spacetime features. We propose a new approach that first detects potential forest disturbances in the pixel-time series based on two-consecutive negative anomalies, and subsequently use space-time features to confirm forest disturbances. The forest disturbance maps generated using the space-time features approach were compared to maps generated using two-consecutive negative anomaly only to detect forest disturbances, and using two-consecutive negative anomalies and the magnitude of change to confirm forest disturbances. We hypothesised that the space-time features approach would produce forest disturbance maps which are spatially more accurate. Throughout this chapter, forest disturbance is defined as forest loss which constitutes either full or partial removal of forest cover, which is visible from Landsat multi-spectral data.

\subsection{Study area}

Our study focused on detecting forest disturbances in the UNESCO Kafa Biosphere Reserve, situated in the southwest of Ethiopia (Figure 4.1), where a moist Afromontane broadleaf evergreen forest with moderate seasonality exists (Devries, Verbesselt, et al. 2015). The forest in this area is subject to smallscale and gradual disturbance processes, caused mainly by small-holder agriculture, human settlements expansion, industrial coffee plantations, and domestic firewood and charcoal extractions (Pratihast et al. 2014; Dresen et al. 2014). Previous studies in the UNESCO Kafa Biosphere Reserve show that separating forest disturbances, especially those involve partial removal of the forest cover, from false detections is difficult (Devries et al. 2016; Devries, Verbesselt, et al. 2015; Schultz et al. 2016). This study area is therefore ideal for evaluating the importance of using space-time features to achieve accurate and timely detection of forest disturbances. We defined forest disturbance as full or partial removal of forest cover, which is visually visible from Landsat multi-spectral images. 


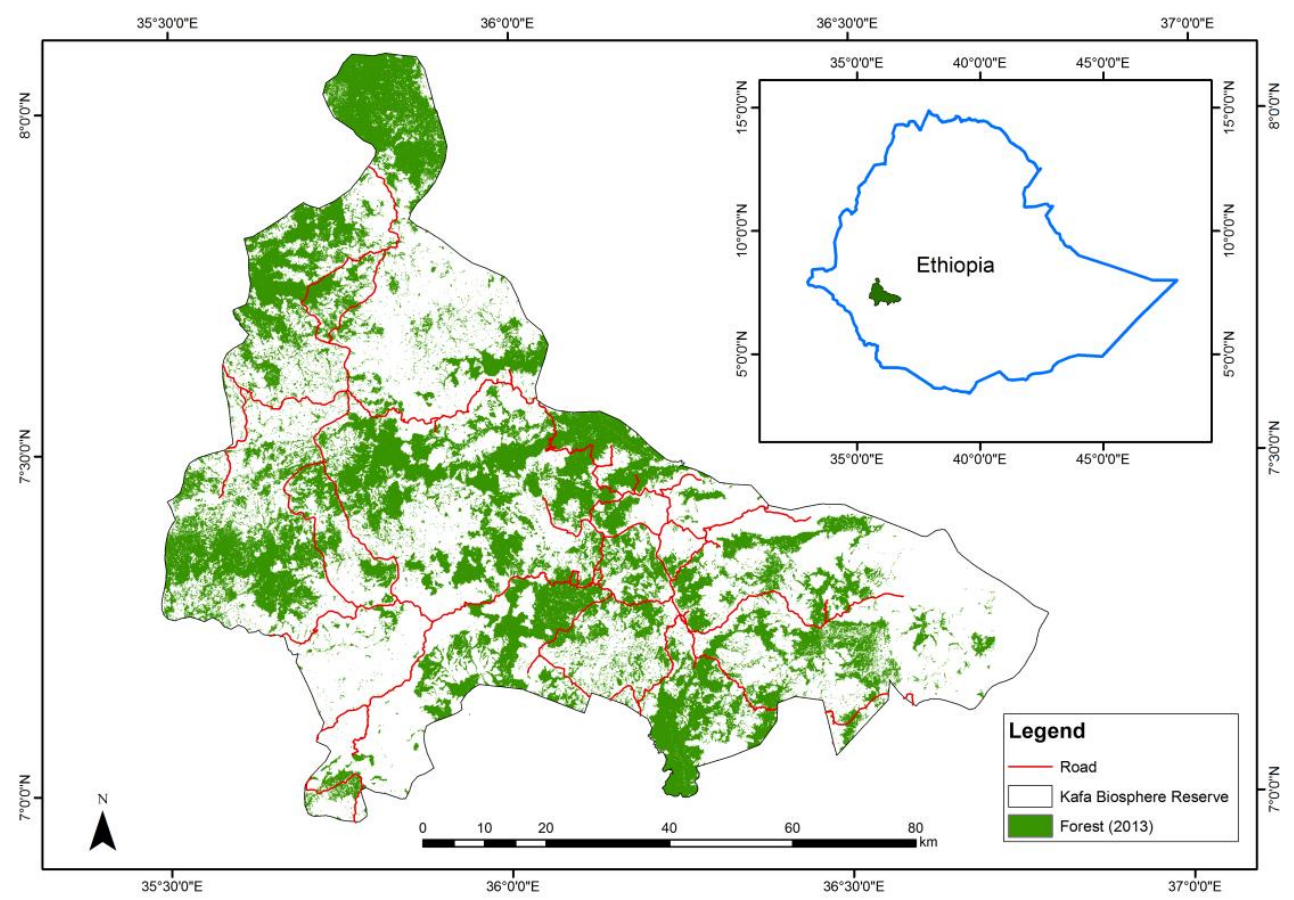

Figure 4.1: Overview of the UNESCO Kafa Biosphere Reserve located in southwest of Ethiopia. Areas in green colour represent the benchmark forest map. The benchmark forest map was generated using a supervised maximum likelihood classifier.

\subsection{Data and methods}

The overview of the data used, and the methods followed in this chapter is shown in Figure 4.2. To detect forest disturbances, we first pre-processed a total of 172 terrain corrected (L1T) multi-spectral images from Landsat-7 Enhanced Thematic Mapper (ETM+, 116 images) and Landsat-8 Operational Land Imager (OLI, 56 images) acquired between January 2010 and June 2016. From these images, we derived normalised difference vegetation index (NDVI; (Tucker 1979; Rouse et al. 1974), Section 4.3.1.1). Second, we produced a benchmark forest mask (Section 4.3.1.2), and used it to mask non-forest areas in the NDVI time series. Third, we normalised NDVI time series spatially to reduce seasonality (Section 4.3.1.3). Fourth, we flagged pixels with two consecutive negative anomalies in Landsat NDVI time series as potentially disturbed (Section 4.3.2). Fifth, we used three different approaches to confirm forest disturbance (Section 4.3.3). In the first approach (Section 4.3.3.1), forest disturbances were immediately confirmed once there were two consecutive 
negative anomalies in the pixel-time series (Two-consecutive anomaly approach). In the second approach (Section 4.3.3.2), we used two consecutive negative anomalies and the magnitude of change to calculate the probability of forest disturbance ( $T_{w o}$ consecutive anomalies + magnitude of change approach). In the third approach (Section 4.3.3.3), we used several space-time features from the local data cube of a flagged pixel to calculate the probability of forest disturbance (Space-time feature approach). Finally, for each approach, we estimated the spatial and temporal accuracy for forest disturbance maps (Section 4.3.4).

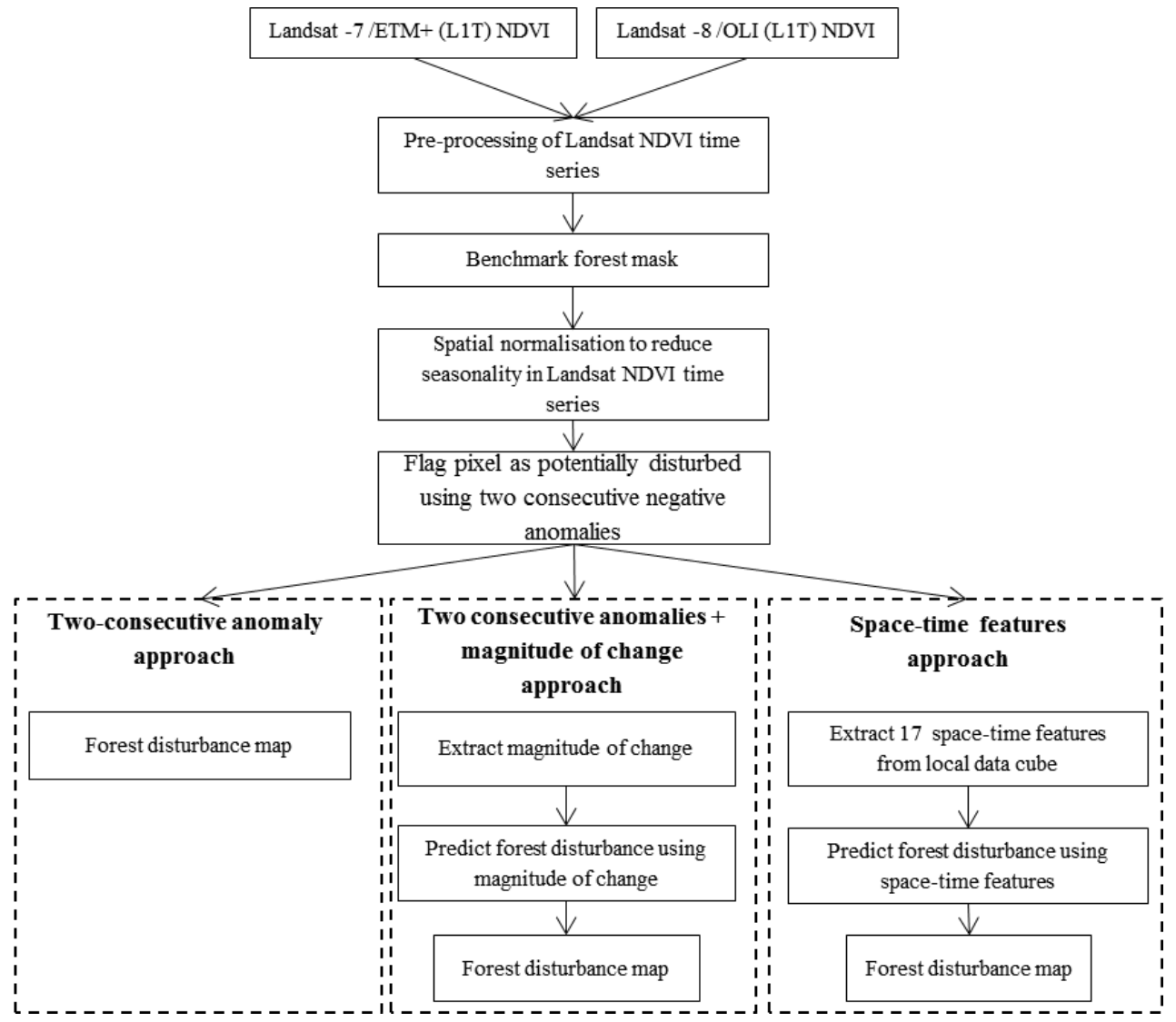

Figure 4.2: The workflow for this chapter. 


\subsubsection{Pre-processing steps}

\subsubsection{Landsat NDVI}

Landsat NDVI time series was derived from terrain corrected (L1T) Landsat-7/ETM+ and Landsat-8/OLI images, spanning from January 2010 to June 2016. Landsat-7/ETM+ and Landsat-8/OLI images, geometrically and atmospherically corrected, were obtained from The United State of America's Geological Survey (USGS) Landsat surface Reflectance (SR) Climate Data Records (CDR; http://landsat.usgs.gov/CDR_LSR.php). USGS use Landsat Ecosystem Disturbance Adaptive Processing System (LEDAPS) algorithm (Masek et al. 2006) to generate Landsat-7/ETM+ surface reflectance products. Landsat 8 OLI surface reflectance products are generated using Landsat 8 OLI surface reflectance algorithm (Vermote et al. 2016). Clouds and cloud shadows in Landsat-7/ETM+ and Landsat-8/OLI images were masked using the CFmask cloud-shadow mask product (Zhu \& Woodcock 2012). CFmask products are distributed together with the Landsat SR CDR Products. Our reference period was 2010 -2013 whereas the monitoring period was 2014 2016.

\subsubsection{Benchmark forest mask}

A benchmark forest mask is essential when monitoring forest cover disturbances from satellite time series to avoid confusing forest cover loss with other land cover dynamics (e.g., cropping cycles; Devries et al., 2015; Hamunyela, Verbesselt, \& Herold, 2016). Similar to (Devries, Verbesselt, et al. 2015) we used a supervised maximum likelihood classifier to generate a forest mask from Landsat-8 OLI mult-spectral image time series for 2013. We removed from the forest mask patches which were smaller than $0.5 \mathrm{~h}$ to conform to areal defining a forest (FAO 2015). The final forest mask product contained 3034809 pixels $(\approx 273133$ ha) classified as forest.

\subsubsection{Spatial normalisation to reduce seasonality in NDVI time series}

The moist Afromontane broadleaf evergreen forest in the UNESCO Kafa Biosphere Reserve exhibits moderate seasonality (Devries, Verbesselt, et al. 2015). If not accounted for appropriately, seasonality may disguise forest disturbances in the time series. Here, we used spatial normalisation (Hamunyela, Verbesselt \& Herold 2016) to reduce seasonality in Landsat NDVI time series. It has been demonstrated that spatial normalisation significantly reduces seasonality (Hamunyela, Verbesselt, \& Herold, 2016; Reiche et al., in review) in satellite time series. Seasonality is reduced by dividing the NDVI 
value for each pixel with the $95^{\text {th }}$ percentile $\left(\mathrm{P}_{95}\right)$ computed from the NDVI values of the pixels within the spatial neighbourhood of such pixel. The spatial window that defines the neighbourhood should be larger than the size of forest disturbance events one wants to detect, to ensure that at least there are forest pixels within the window to avoid normalising against a value from disturbed area (Hamunyela, Verbesselt, Bruin, et al. 2016). The spatial window of 15 by 15 pixels $(\approx 20$ ha) we used in this study was deemed sufficient because forest disturbance patches in the UNESCO Kafa Biosphere Reserve tend to be small (Devries, Verbesselt, et al. 2015; Pratihast et al. 2014).

\subsubsection{Detecting negative anomalies in Landsat NDVI data cubes}

We detected negative anomalies in the local data cubes of spatially normalised Landsat NDVI time series using a method that detects forest disturbances as extreme events in Landsat data cubes (Hamunyela, Verbesselt, Bruin, et al. 2016). The local data cube is defined around each pixel in the image time series and its temporal and spatial extents are user-defined (Hamunyela, Verbesselt, Bruin, et al. 2016). We used a local data cube with a spatial extent of the $15 \times 15$ Landsat pixels. The temporal extent was from 2010 to 2016. The local data cube is split into a reference cube and monitoring cube. The reference cube contains historical observations, whereas the monitoring cube contains newly acquired observations that need to be assessed for forest disturbance (Hamunyela, Verbesselt, Bruin, et al. 2016). Negative anomalies in the monitoring period were identified using a threshold computed from the observations available in the reference period of the local data cube. The threshold corresponds to a user-specified percentile (e.g. $5^{\text {th }}$ percentile), and an observation was a negative anomalies if it was below the specified percentile (Zscheischler et al. 2013). Here, we evaluated five percentile thresholds ( $1^{\text {st }}, 2^{\text {nd }}, 3^{\text {rd }}, 4^{\text {th }}$ and $\left.5^{\text {th }}\right)$. A pixel was flagged as potentially disturbed if it had two consecutive negative anomalies in its time series. Three different approaches were used to confirm negative anomalies as forest disturbance (Section 4.3.3). Observations in the monitoring period were assessed sequentially rather than simultaneously to mimic a near real-time monitoring scenario.

\subsubsection{Confirming anomalies as forest disturbances}

\subsubsection{Two-consecutive anomaly approach}

With this approach, a pixel was immediately confirmed as disturbed once two consecutive negative anomalies in the pixel-time series. This approach was proposed in our earlier work, and it has been demonstrated at two sites, one in 
dry tropical forest in Bolivia and the other in humid evergreen forest in Brazil (Hamunyela, Verbesselt, Bruin, et al. 2016).

\subsubsection{Two consecutive anomalies + magnitude of change approach}

We used the two consecutive negative anomalies $\left(\mathrm{C}_{\text {anomaly }}\right)$ and magnitude of change $\left(\mathrm{M}_{\text {change }}\right)$ (Table 1) to confirm forest disturbance. The aim here was to assess whether using magnitude of change together with two consecutive negative anomalies would improve discrimination of forest disturbances from false detections when compared to a scenario whereby forest disturbance is confirmed using two consecutive negative anomalies only. We used a random forest model $(\mathrm{n}=1311$, trees $=501$; $($ Breiman 2001) $)$ to calculate the probability of disturbance using two consecutive negative anomalies and magnitude of change as predictors of forest disturbance. We used a probability 0.5 as threshold for accepting negative anomalies as forest disturbance. Random forest model was trained using a training data set acquired through visual interpretation of Landsat multi-spectral images. The training sample pixels were selected through probability sampling, using a forest disturbance map generated using the two-consecutive anomaly approach (Section 4.3.3.1).

\subsubsection{Space-time feature approach}

We used all 17 space-time features (see Table 4.1) extracted from the local data cube of Landsat NDVI time series, to calculate the probability of forest disturbance once two consecutive anomalies are signalled at a pixel. The probability of forest disturbance was calculated using a trained a random forest model $(n=1311$, trees $=500 ;($ Breiman 2001) $)$. A pixel was declared disturbed if the probability of disturbance was equal or greater than 0.5. The importance of each space-time feature for predicting forest disturbance was determined using the conditional variable importance approach (Strobl et al. 2008) that accounts for correlation between variables, and accommodates variables of different data types. We used the same training data set as in Section 4.3.3.2 to train the random forest model.

Out of 17 features, one $\left(\mathrm{SD}_{\mathrm{rc}}\right)$ is measuring spatio-temporal variability in the reference period, three ( $\left.\mathrm{Pocum}_{\mathrm{c}}, \mathrm{Pr}_{\text {cum }}, \mathrm{SD}_{\text {Trend }}\right)$ are measuring change in spatial variability over time, six (Poextremes, $\mathrm{Popatch}_{\text {postep }}, \mathrm{Pr}_{\mathrm{patch}}, \mathrm{Pr}_{\text {extremes }}, \mathrm{Pr}_{\text {step }}$ ) are measuring spatio-temporal association with pixels experiencing negative anomalies, and three $\left(\mathrm{CB}_{\mathrm{nf}}, \mathrm{N}_{\mathrm{nf}}, \mathrm{P}_{\mathrm{nf}}\right)$ are measuring spatial association with non-forest areas. The remaining four features, i.e. $\mathrm{V}_{\mathrm{rc}}, \mathrm{Q}_{\text {thresh, }} \mathrm{M}_{\text {change, }} \mathrm{C}_{\text {anomaly }}$, represent the number of observations in the local data cube over the reference period, the anomaly threshold for identifying negative anomalies, the magnitude of change and two consecutive negative anomalies, respectively. 
Spatial variability and spatio-temporal variability can be measured using various metrics (e.g. the range, standard deviation; (Ichoku et al. 2002; Glasbey et al. 2001; Uddstrom \& Oien 1999)). Here, we used the standard deviation as a--measure of spatial variability and spatio-temporal variability. Spatial variability was calculated at each time slice within the local data cube, whereas spatio-temporal variability was calculated by taking in account both the spatial and temporal information in the local data cube. Many space-time features can be extracted from data cubes of satellites image time series. In this study, we chose the space-time features that can be extracted easily in an automated manner from local data cube to ensure that the change detection framework can be used for near real-time forest change monitoring.

Table 4.1: Space-time features extracted from local data cubes for Landsat NDVI time series to predict the probability of forest disturbances given two consecutive negative anomalies at particular pixel. The spatial extent of the local data cube was 15 by 15 Landsat pixels. $\mathrm{T}_{1}$ refers to the time step where the first negative anomaly of the two consecutive negative anomalies is detected, whereas $\mathrm{T}_{2}$ is time step for the second negative anomaly. The spacetime features are grouped by category in the table

\begin{tabular}{|c|c|c|c|c|}
\hline Category & Feature & Acronym & Data type & Explanation \\
\hline \multirow{2}{*}{ 胥 } & $\begin{array}{l}\text { Consecutive } \\
\text { anomalies }\end{array}$ & $\mathrm{C}_{\text {anomaly }}$ & $\begin{array}{l}\text { Discrete- } \\
\text { Numeric }\end{array}$ & $\begin{array}{l}\text { Number of consecutive } \\
\text { negative anomalies. Here, } \\
\text { we used two consecutive } \\
\text { negative anomalies }\end{array}$ \\
\hline & $\begin{array}{l}\text { Magnitude of } \\
\text { change }\end{array}$ & $\mathrm{M}_{\text {change }}$ & Continuous & $\begin{array}{l}\text { Magnitude of change } \\
\text { calculated by subtracting } \\
\text { the anomaly threshold from } \\
\text { the second anomaly at } \mathrm{T}_{2}\end{array}$ \\
\hline \multirow{3}{*}{ 丞 } & $\begin{array}{l}\text { Number of non- } \\
\text { forest pixels in } \\
\text { the reference } \\
\text { period }\end{array}$ & $\mathrm{CB}_{\mathrm{nf}}$ & $\begin{array}{l}\text { Discrete- } \\
\text { Numeric }\end{array}$ & $\begin{array}{l}\text { Number of pixels within the } \\
\text { local data cube which have } \\
\text { been masked as non-forest } \\
\text { in the reference period }\end{array}$ \\
\hline & $\begin{array}{l}\text { Presence of } \\
\text { non-forest } \\
\text { neighbours }\end{array}$ & $\mathrm{N}_{\mathrm{nf}}$ & $\begin{array}{l}\text { Binary }(\text { yes } \\
=1, \text { no }=0)\end{array}$ & $\begin{array}{l}\text { Indicates whether any of the } \\
8 \text {-connected neighbours for } \\
\text { the focal pixel is already } \\
\text { non-forest in the reference } \\
\text { period }\end{array}$ \\
\hline & $\begin{array}{l}\text { Number non- } \\
\text { forest }\end{array}$ & $\mathrm{P}_{\mathrm{nf}}$ & $\begin{array}{l}\text { Discrete- } \\
\text { Numeric }\end{array}$ & $\begin{array}{l}\text { Number of the } 8 \text {-connected } \\
\text { neighbours for the focal }\end{array}$ \\
\hline
\end{tabular}




\begin{tabular}{|c|c|c|c|c|}
\hline & neighbours & & & $\begin{array}{l}\text { pixel which are already non- } \\
\text { forest in the reference } \\
\text { period }\end{array}$ \\
\hline & $\begin{array}{l}\text { Presence of } \\
\text { neighbours } \\
\text { with negative } \\
\text { anomalies at } \mathrm{T}_{1}\end{array}$ & $\operatorname{Pr}_{\text {step }}$ & $\begin{array}{l}\text { Binary } \quad(\text { yes } \\
=1, \text { no }=0)\end{array}$ & $\begin{array}{l}\text { Indicates whether any of the } \\
\text { 8-connected neighbours for } \\
\text { the focal pixel also } \\
\text { experienced negative } \\
\text { anomalies at } \mathrm{T}_{1}\end{array}$ \\
\hline & $\begin{array}{l}\text { Number of } \\
\text { neighbours } \\
\text { with negative } \\
\text { anomalies at } \mathrm{T}_{1}\end{array}$ & $\operatorname{Pr}_{\text {patch }}$ & $\begin{array}{l}\text { Discrete- } \\
\text { Numeric }\end{array}$ & $\begin{array}{l}\text { Number of } 8 \text {-connected } \\
\text { neighbours for focal pixel } \\
\text { which also experienced } \\
\text { negative anomalies at } \mathrm{T}_{1}\end{array}$ \\
\hline & $\begin{array}{l}\text { Number of } \\
\text { pixels in the } \\
\text { local data cube } \\
\text { with negative } \\
\text { anomalies at } \mathrm{T}_{1}\end{array}$ & $\mathrm{Pr}_{\text {extremes }}$ & $\begin{array}{l}\text { Discrete- } \\
\text { Numeric }\end{array}$ & $\begin{array}{l}\text { Number of pixels in the } \\
\text { local data cube which also } \\
\text { experienced negative } \\
\text { anomalies at } \mathrm{T}_{1}\end{array}$ \\
\hline & $\begin{array}{l}\text { Presence of } \\
\text { neighbours } \\
\text { with negative } \\
\text { anomalies at } \mathrm{T}_{2}\end{array}$ & $\mathrm{Po}_{\text {step }}$ & $\begin{array}{l}\text { Binary } \quad(\text { yes } \\
=1, \text { no }=0)\end{array}$ & $\begin{array}{l}\text { Indicates whether any of the } \\
8 \text {-connected neighbours of } \\
\text { the focal pixel is also } \\
\text { experiencing negative } \\
\text { anomalies at } \mathrm{T}_{2}\end{array}$ \\
\hline & $\begin{array}{l}\text { Number of } \\
\text { neighbours } \\
\text { with anomalies } \\
\mathrm{T}_{2}\end{array}$ & Popatch & $\begin{array}{l}\text { Discrete- } \\
\text { Numeric }\end{array}$ & $\begin{array}{l}\text { Number of } 8 \text {-connected } \\
\text { neighbours of the focal pixel } \\
\text { which are also experiencing } \\
\text { negative anomalies at } \mathrm{T}_{2}\end{array}$ \\
\hline & $\begin{array}{l}\text { Number of } \\
\text { pixels with } \\
\text { negative } \\
\text { anomalies in } \\
\text { the local data } \\
\text { cube at } \mathrm{T}_{2}\end{array}$ & $\mathrm{Po}_{\text {extremes }}$ & $\begin{array}{l}\text { Discrete- } \\
\text { Numeric }\end{array}$ & $\begin{array}{l}\text { Number of pixels in local } \\
\text { data cube with negative } \\
\text { anomalies at } \mathrm{T}_{2}\end{array}$ \\
\hline 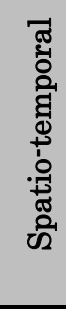 & $\begin{array}{l}\text { Cumulative } \\
\text { sum of } \\
\text { residuals for } \\
\text { spatial } \\
\text { variability at } \mathrm{T}_{2}\end{array}$ & $\mathrm{Po}_{\text {cum }}$ & Continuous & $\begin{array}{l}\text { Cumulative sum of } \\
\text { residuals for spatial } \\
\text { variability at } \mathrm{T}_{2} \text {. Calculated } \\
\text { following these steps: i) } \\
\text { calculate spatial variation } \\
\text { (standard deviation) at each } \\
\text { time step of the time series; } \\
\text { ii) calculate the temporal }\end{array}$ \\
\hline
\end{tabular}




\begin{tabular}{|c|c|c|c|}
\hline & & & $\begin{array}{l}\text { mean of spatial variation; } \\
\text { (3) subtract the temporal } \\
\text { mean of spatial variation } \\
\text { from spatial variation at } \\
\text { each time step }\end{array}$ \\
\hline $\begin{array}{l}\text { Cumulative } \\
\text { sum of } \\
\text { residuals for } \\
\text { spatial } \\
\text { variability at } \mathrm{T}_{1}\end{array}$ & $\operatorname{Pr}_{\text {cum }}$ & Continuous & $\begin{array}{l}\text { Cumulative sum of } \\
\text { residuals for spatial } \\
\text { variability at } T_{1} \text {. Calculated } \\
\text { in same way as } \text { Pocum }_{\text {cobove }}\end{array}$ \\
\hline $\begin{array}{l}\text { Anomaly } \\
\text { threshold }\end{array}$ & Qthresh & Continuous & $\begin{array}{l}\text { Threshold for identifying } \\
\text { negative anomalies, } \\
\text { computed from the local } \\
\text { data cube over the reference } \\
\text { period, corresponding to the } \\
\text { specified percentile (e.g. } 5^{\text {th }} \\
\text { percentile) }\end{array}$ \\
\hline $\begin{array}{l}\text { Variability of } \\
\text { observations in } \\
\text { the local data } \\
\text { cube in the } \\
\text { reference period }\end{array}$ & $\mathrm{SD}_{\mathrm{rc}}$ & Continuous & $\begin{array}{l}\text { Standard deviation of the } \\
\text { observations in the } \\
\text { reference period of a local } \\
\text { data cube }\end{array}$ \\
\hline $\begin{array}{l}\text { Temporal } \\
\text { linear trend in } \\
\text { spatial } \\
\text { variability }\end{array}$ & $\mathrm{SD}_{\text {Trend }}$ & Continuous & $\begin{array}{l}\text { Temporal linear trend in } \\
\text { spatial variability, } \\
\text { quantified using a linear } \\
\text { model. }\end{array}$ \\
\hline $\begin{array}{l}\text { Number of } \\
\text { observations in } \\
\text { local data cube } \\
\text { over the } \\
\text { reference period }\end{array}$ & $\mathrm{V}_{\mathrm{rc}}$ & $\begin{array}{l}\text { Discrete- } \\
\text { Numeric }\end{array}$ & $\begin{array}{l}\text { Number of valid } \\
\text { observations in the local } \\
\text { data cube over the reference } \\
\text { period }\end{array}$ \\
\hline
\end{tabular}

\subsubsection{Estimating the spatial and temporal accuracy}

We estimated the spatial and temporal accuracy for forest cover disturbance using 2343 sample pixels generated through stratified probability sampling (Stehman 2009). The sample pixels and the strata were generated using a forest disturbance map produced by Hamunyela et al (in preparation). This forest disturbance map was generated using the change detection framework presented in this chapter, but it was based on the combination of 
Landsat and Sentinel-2 data. The area proportion for each stratum is shown in Table 4.2. The largest stratum (No change I) represents forest areas which were $60 \mathrm{~m}$ away from areas where disturbances were detected. Stratum No change II was a $60 \mathrm{~m}$ buffer around the areas where disturbances were detected. A buffer zone was created around disturbances in order to improve the estimation of the omission error because changes and misclassifications are expected at the edges of the forest. Stratum Change I represents areas where disturbances were detected between 2014 and 2015, while stratum Change II represents areas where disturbances were detected between 2015 and 2016 . The area was classified as change if the probability of forest disturbance was greater than or equal to 0.5. The allocation of sample size to each stratum was based on the approach recommended by (Olofsson et al. 2014; Stehman 2012) that ensures a reliable estimation of overall accuracy, producer's and user's accuracy for classes with small area proportions. Based on this sample allocation, we calculated the area-adjusted overall accuracy, producer's accuracy and user's accuracy. In addition, we calculated the area bias (a difference between user's and producer's accuracy), and the temporal accuracy expressed as a median temporal detection delay in days. The temporal detection delay was defined as the number of days between the date of disturbance as per reference data and the acquisition date for the image in which a disturbance was confirmed. Similar to (Cohen et al. 2010; Devries, Verbesselt, et al. 2015; Dutrieux et al. 2015; Hamunyela, Verbesselt \& Herold 2016; Zhu \& Woodcock 2014b), reference data were collected from Landsat multi-spectral images through visual interpretation of images. This approach, however, has major limitation because forest disturbances which are smaller than the spatial resolution of Landsat images might not be visible, but might be detected. It is for this reason that we complemented the visual interpretation of Landsat images with very high resolution imagery available in Google Earth (https://www.google.com/earth/) and Bing Maps (http://www.bing.com/maps/), but images are not temporally dense like Landsat.

Table 4.2: Area proportions, number of pixels and allocated sample size for each stratum

\begin{tabular}{lccc}
\hline Stratum & No. of pixels & Area proportion & No. of samples \\
\hline No change I & 2694771 & 0.888 & 1443 \\
No Change II & 261204 & 0.086 & 500 \\
Change I & 71167 & 0.023 & 200 \\
Change II & 7667 & 0.003 & 200 \\
\hline Total & 3034809 & 1 & 2343 \\
\hline
\end{tabular}




\subsubsection{Assessing the effect of probability threshold on spatial accuracy}

In Section 4.3.3.2 and 4.3.3.3, we used a probability threshold of 0.5 to discriminate false detections from real changes. In some instances, the user may have a different monitoring objective. For example, a user may prefer a map with the lowest area bias. We assessed how the probability threshold affects the spatial accuracy for forest disturbance maps produced using the space-time feature approach and the magnitude of change approach. We chose the maps which were generated using the $5^{\text {th }}$ percentile that achieved the highest producer's accuracy and the shortest temporal detection delay. More specifically, we assessed how overall accuracy, user's accuracy, producer's accuracy vary as function of the probability of threshold for accepting negative anomalies as forest disturbance. We increased the probability threshold from 0 to 0.95 , at an interval of 0.05 .

\section{$4.4 \quad$ Results}

The accuracies for the space-time feature approach and the two consecutive anomalies + magnitude of change approach are based on the forest disturbance maps generated using 0.5 as the probability threshold for accepting anomalies as forest disturbance. Effect of the probability threshold on the overall accuracy, user's accuracy and producer's accuracy for maps generated using the space-time features approach and the two consecutive anomalies + magnitude of change approach is shown in Section 4.4.2. In Section 4.4.3, the area estimates for disturbed forest are presented. The ranking of the space-time features based on their conditional variable importance are presented in Section 4.4.4.

\subsubsection{Spatial and temporal accuracy}

Using the space-time feature approach yielded the highest user's accuracy across all the percentile thresholds ( $>70 \%$, Figure $4.3 \mathrm{~d})$. More specifically, for each percentile threshold, the user's accuracy achieved from the space-time feature approach was at least $26 \%$ higher than the user's accuracy achieved either from a two-consecutive anomaly approach or from the magnitude of change approach. This gain in the user's accuracy was particularly high (36.4\%) when using the least strict percentile threshold ( $5^{\text {th }}$ percentile). The difference in the user's accuracy between the two-consecutive anomaly approach and the two consecutive anomalies + magnitude of change approach was marginal across all the percentile thresholds. Across all percentile thresholds, the user's accuracy achieved from the two-consecutive anomaly approach and the two consecutive anomalies + magnitude of change approach 
was below $60 \%$. Compared to the space-time feature approach, the twoconsecutive anomaly approach and the two consecutive anomalies + magnitude of change approach achieved the higher producer's accuracy when using the least strict percentile thresholds ( $4^{\text {th }}$ and $5^{\text {th }}$ percentile, Figure $4.3 \mathrm{c}$ ), but the space-time feature approach achieved the highest overall accuracy (Figure $4.3 \mathrm{a}$ ), and the lowest area bias (Figure 4.3b). More specifically, the producer's accuracy for the space-time feature approach was about $9 \%$ lower than those of the two-consecutive anomaly approach and the two consecutive anomalies + magnitude of change approach when using the least strict percentile threshold. For all approaches, the least strict percentiles achieved the shortest temporal detection delay (32 days) and highest producer's accuracy (> 79\%). For the space-time feature approach, the differences in the producer's accuracy and median temporal detection delay between the most strict percentile threshold ( $5^{\text {th }}$ percentile) and least strict percentile threshold ( $1^{\text {st }}$ percentile) were $47.7 \%$ and 137 days, respectively. Figure 4.4 shows an example of a false detection, related to remnant clouds, which was accepted when using the two-consecutive anomaly approach and the magnitude of change approach, but rejected by the space-time feature approach. 

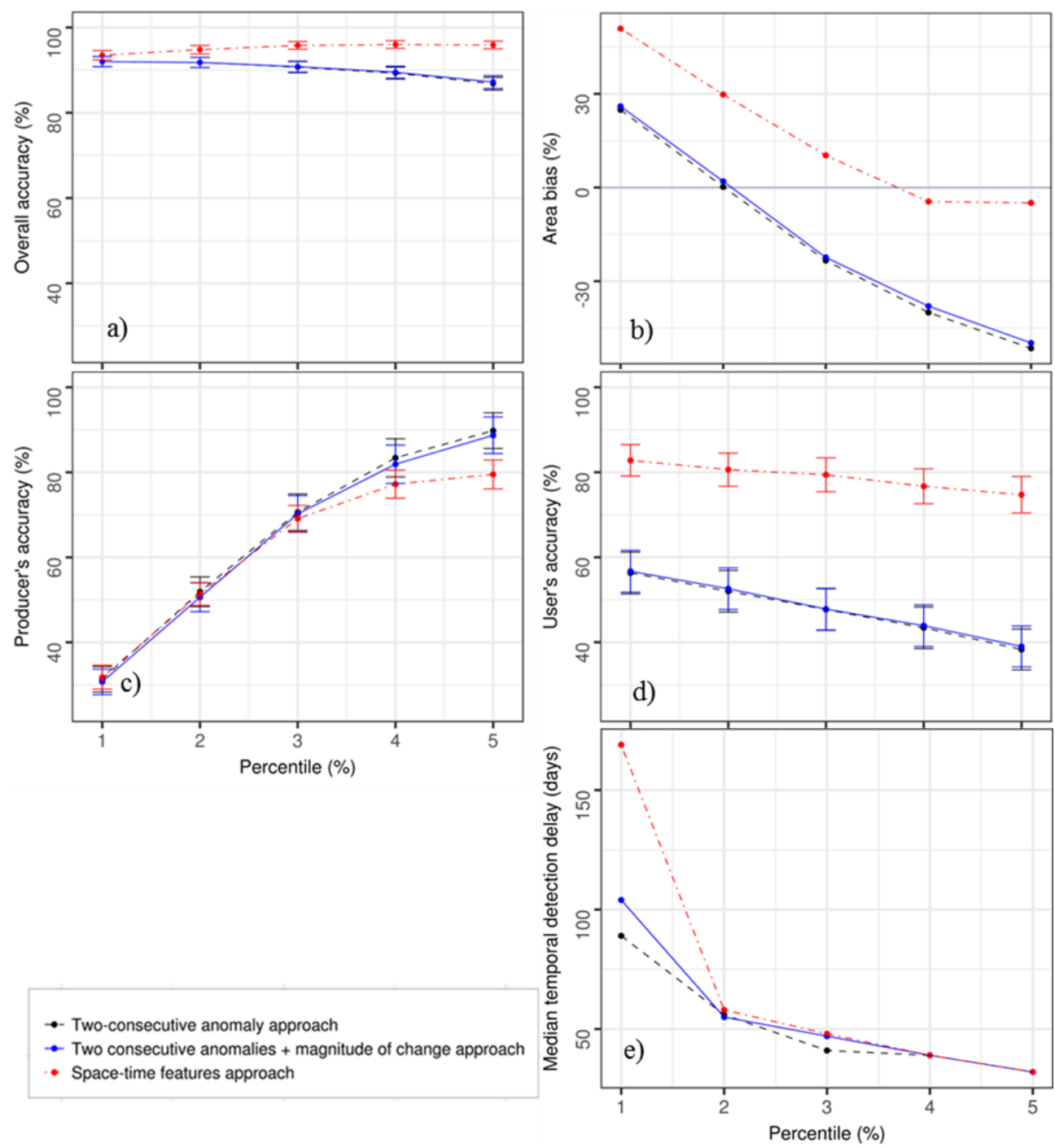

Figure 4.3: Overall accuracy (a); area bias (b); producer's accuracy (c); user's accuracy (d); and median temporal detection delay (e) for forest disturbances detected in the UNESCO Kafa Biosphere Reserve between 2014 and 2016 from Landsat NDVI time series. Forest disturbances were confirmed using three approaches: the two-consecutive anomaly approach, the two consecutive anomalies + magnitude of change approach and the space-time feature approach. The accuracies are shown as a function for the percentile thresholds for declaring anomalous observations in local data cubes. The vertical bars in (a), (c) and (d) indicate the $95 \%$ confidence interval. 


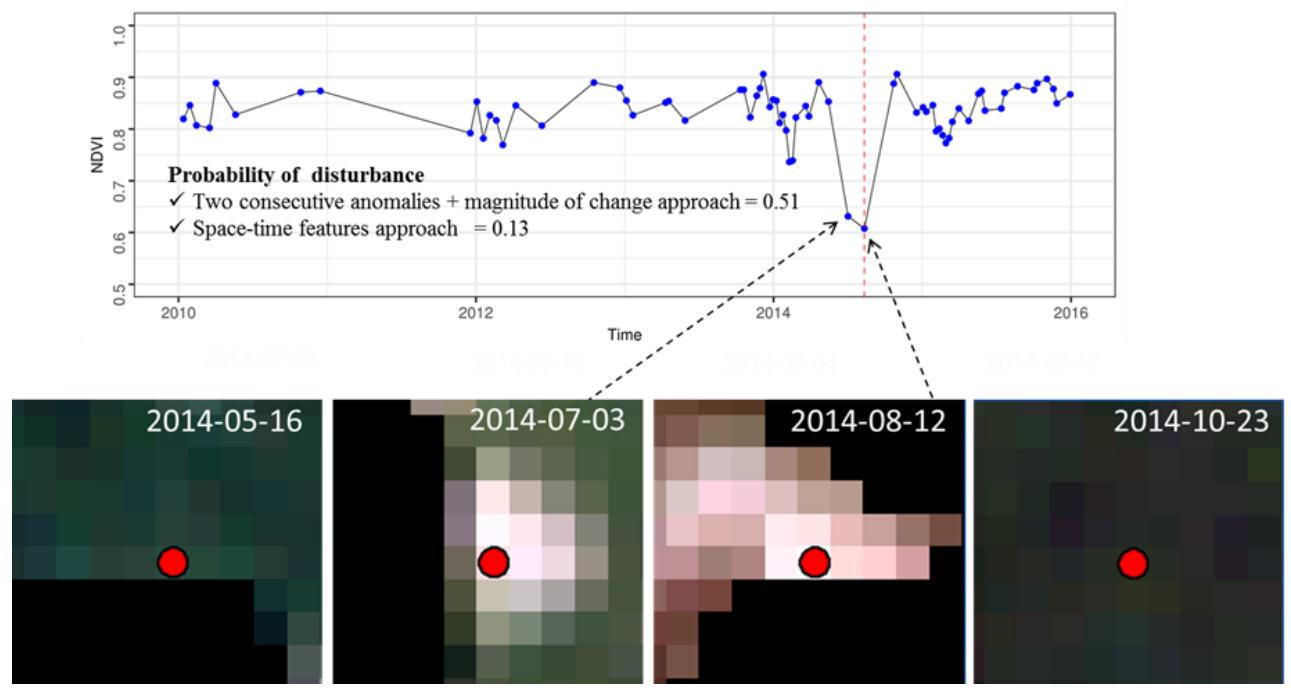

Figure 4.4: An example of a false detection (red dotted line), related to remnant clouds, which was rejected (probability of disturbance $=0.13$ ) when using the space-time features. The probability of disturbance was 0.51 when only using the magnitude of change to predict forest disturbance. The magnitude of change was -0.22 . The red dot represents the pixel (location: $7^{\circ} 30^{\prime} 5.042 " \mathrm{~N}$, $36^{\circ} 1^{\prime} 30.959^{\prime \prime} \mathrm{E}$ ) whose time series is shown, and the black areas in the image chips represent areas which were masked as clouds or cloud shadow using CFmask. Image chips which were covered completely with no data are skipped.

\subsubsection{The effect of the probability threshold on spatial accuracy}

For the space-time approach, the user's accuracy for map produced using the $5^{\text {th }}$ percentile threshold increased by $69.8 \%$, reaching $99.1 \%$ when increasing the probability threshold from 0 to 0.95 . The producer's accuracy decreased by $75.8 \%$, reaching $15.4 \%$. The crossover point for the user's and producer's accuracy was approximately at the probability threshold of 0.55 (Figure 4.5). At this probability threshold, the user's accuracy was $78.3 \%$ while the producer's accuracy was $76.8 \%$. In contrast, the user's and producer's accuracy did not reach the crossover point when using the two consecutive anomalies + magnitude of change approach (Figure 4.5). For this approach, the lowest area bias $(17.3 \%)$ was achieved at the probability threshold of 0.95 , and it was in favour of the producer's accuracy. For the magnitude of change approach, the user's accuracy only increased by $18.3 \%$, reaching $47.6 \%$ when increasing the probability threshold from 0 to 0.95 ; the producer's accuracy decreased by $26.3 \%$, reaching $64.3 \%$. 


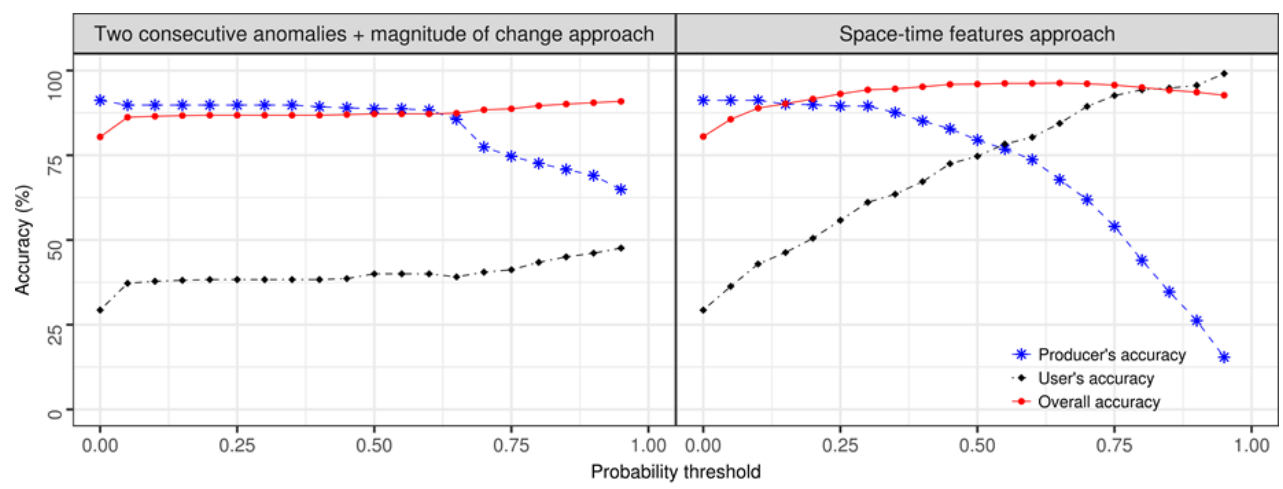

Figure 4.5: Overall accuracy, producer's accuracy and user's accuracy for forest disturbances detected in the UNESCO Kafa Biosphere Reserve between 2014 and 2016 from Landsat NDVI time series using the $5^{\text {th }}$ percentile while changing the probability threshold for accepting two consecutive negative anomalies as forest disturbance.

\subsubsection{Area estimates for disturbed forest}

Using the map produced from the space-time feature approach, the $5^{\text {th }}$ percentile threshold and probability threshold of 0.55 (Figure 4.6), we estimated that about 17915 ha of forest in UNESCO Kafa Biosphere Reserve has been disturbed between 2014 and 2016. The disturbances involved either full or partial removal of forest canopy. Disturbances were mainly concentrated at edges of forest (Figure 4.6). 


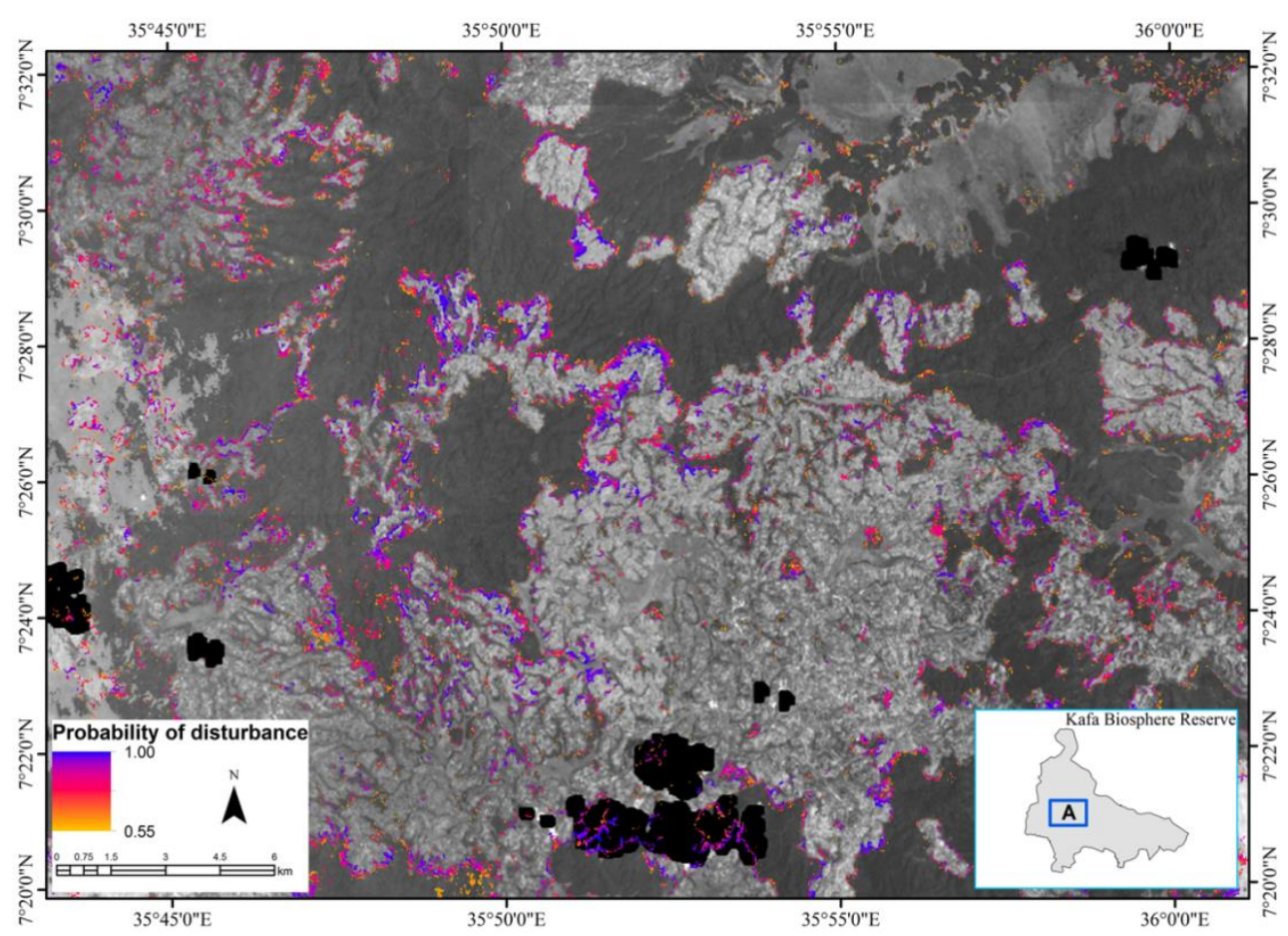

Figure 4.6: An example of forest disturbances detected in the UNESCO Kafa Biosphere Reserve between 2014 and 2016 from Landsat NDVI time series (insert A). The probability disturbance was calculated using the space-time features extracted from Landsat NDVI data cubes based on $5^{\text {th }}$ percentile threshold and 0.55 probability threshold. The base image is the surface reflectance in the red channel (Band 4) for Landsat-8/OLI acquired on 10-032016. The black patches represent areas masked for clouds and cloud shadows.

\subsubsection{Space-time features important for predicting forest disturbance}

Our results show that cumulative sums of residuals for spatial variability (Pocum, and $\operatorname{Pr}_{\text {cum }}$ ), the anomaly threshold for identifying anomalous observations ( $Q_{\text {thresh }}$ ), the variability in the reference cube of a local data cube $\left(\mathrm{SD}_{\mathrm{rc}}\right)$ and the number of non-forest pixels within the local data cube in the reference period $\left(\mathrm{CB}_{\mathrm{nf}}\right)$ were the most important predictors for forest disturbance (Figure 4.7). These space-time features were two times more important than consecutive negative anomalies $\left(\mathrm{C}_{\text {anomaly }}\right)$ and the magnitude of change ( $\left.\mathrm{M}_{\text {change }}\right)$. 


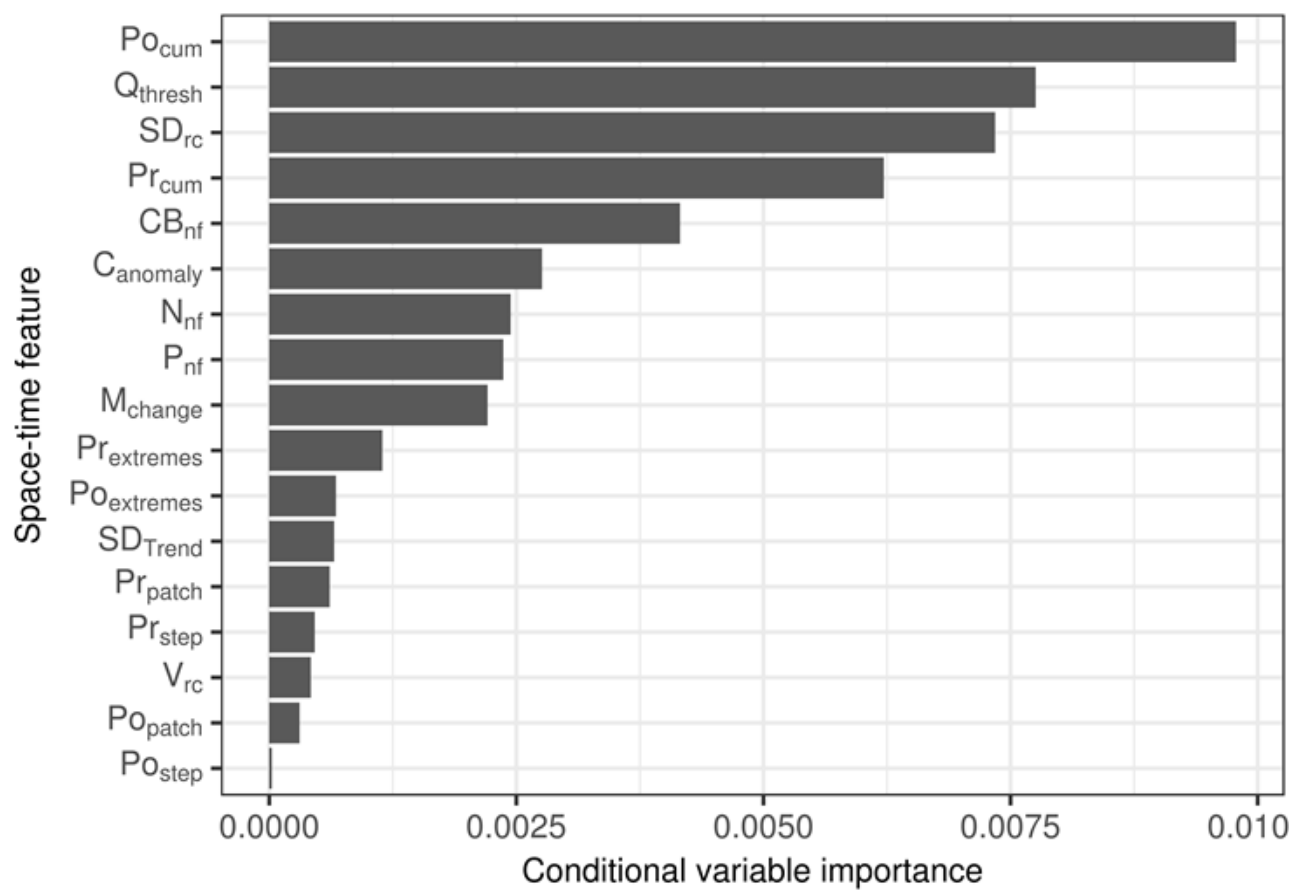

Figure 4.7: Conditional variable importance for each space-time feature using to predict forest disturbances in the UNESCO Kafa Biosphere Reserve from data cubes of Landsat normalised difference vegetation index (NDVI) time series. Training data $(\mathrm{n}=1311)$ was used to determine the conditional variable importance.

\subsection{Discussion}

In this chapter, we proposed to use space-time features to improve forest change monitoring using Landsat time series. Space-time features are used to confirm forest disturbances when negative anomalies are detected in the pixeltime series. Our results show that using space-time features to confirm forest disturbances reduces false detections significantly, with minimal negative effect on producer's accuracy for forest disturbance. Our results also show that using two consecutive negative anomalies to confirm forest disturbances does not reduce false detections sufficiently even when using strict percentile thresholds to identify the anomalies. Instead of reducing false detections, strict percentile thresholds amplified the omission error, and led to a longer temporal detection delay. These results suggest that the criterion of two consecutive anomalies is not robust enough to reject false detection as previously thought (Hamunyela, Verbesselt, Bruin, et al. 2016; Zhu et al. 2012; Reiche, de Bruin, 
et al. 2015). Two consecutive anomalies might be sufficient in areas where forest disturbances occur at large scale and in an abrupt manner [e.g. 6,7], but not sufficient when dealing with complex small-scale forest disturbances (Devries, Verbesselt, et al. 2015; Pratihast et al. 2014). Our results also show that using the magnitude of change, in addition two consecutive negative anomalies, to confirm forest disturbances does not reduce false detections sufficiently. The two consecutive anomalies + magnitude of change approach produced spatial and temporal accuracies largely similar to those of the twoconsecutive anomalies approach. The false detections remained high despite using strict probability thresholds, implying that false detections also had high probability of disturbance when using magnitude of change as a predictor of forest disturbance. Overall, these findings suggest that temporal information alone might not be sufficient to reduce false detections especially when aiming for accurate and timely detection of small-scale and gradual forest disturbances.

We observed that forest disturbances were mainly detected on the periphery of the forest. This pattern of forest disturbance is consistent with the findings of the previous studies (Devries, Verbesselt, et al. 2015; Devries et al. 2016) that mapped forest disturbances in this area using Landsat time series. The drivers of forest disturbance, especially expansion of small-holder agricultural areas and human settlements are likely to occur on edges of the forest especially when there has been an ongoing forest conservation and restoration activities in the area because it might be easier to illegally disturb forest edges than clearing new large intact forest area.

\subsubsection{Importance of space-time features to accurate and timely detection of forest disturbances}

Space-time features provided an opportunity to timely detect anomalies without amplifying false detections. This capacity to reject many false detections explains why the difference in the user's accuracy between the maps produced using the least strict percentile threshold ( $5^{\text {th }}$ percentile) and the maps produced using most strict percentile threshold ( $1^{\text {st }}$ percentile) was only (8\%). This difference was greater than $17 \%$ when using only two consecutive negative anomalies or magnitude of change to confirm forest disturbances. These results suggest that, although using less strict percentile thresholds may initially flag many pixels as potentially disturbed, a set of space-time features provides sufficient information to accurately discriminate false detections from real changes. At the same time, by using least strict percentile thresholds, we can reduce the omission error and the temporal detection delay. When aiming for the lowest area bias (1.5\%), our spatial accuracies (overall accuracy = $96.2 \%$, user's accuracy $=76.8 \%$, producer's accuracy $=78.3 \%$ ) were within the range of those reported in other studies that attempted to map forest 
disturbances at annual or sub-annual scales in recent years (Reiche, Verbesselt, et al. 2015; Grogan et al. 2015; Hamunyela, Verbesselt \& Herold 2016; Hamunyela, Verbesselt, Bruin, et al. 2016; Dutrieux et al. 2015; Reiche, de Bruin, et al. 2015; Devries, Verbesselt, et al. 2015). However, our high spatial accuracies were not achieved at the expense of temporal accuracy.

\subsubsection{Important space-time features for forest disturbance predicting}

Our analysis for conditional variable importance shows that space-time features which are related to spatial variability were the most important predictors for forest disturbances. More specifically, cumulative sum of residuals for spatial variability at the time step before the focal pixel is flagged as potentially disturbed, the anomaly threshold, the variability of the observations in the reference cube, and the cumulative sum of residuals for spatial variability at the time step the focal pixel is flagged as potentially disturbed were the most important predictors for forest disturbance. All these variables are computed using both spatial and temporal information, and are measuring spatio-temporal variability. The high importance of the variables related to spatio-temporal variability is not surprising because spatio-temporal variability is likely to increase once the forest become disturbed. The number of non-forest pixels within the local data cube was also an important predictor of forest disturbances. This is expected because new human-induced forest disturbance typically occur closer to old disturbances in time and space (Alves 2002). So negative anomalies detected at forest pixels which have spatial association with non-forest areas are most likely to signify forest disturbance. Magnitude of change and two consecutive negative anomalies, which are widely used to distinguish real changes from false detections (Hamunyela, Verbesselt \& Herold 2016; Hamunyela, Verbesselt, Bruin, et al. 2016; Devries, Verbesselt, et al. 2015; Zhu et al. 2012; Reiche, de Bruin, et al. 2015), were not the strongest predictors for forest disturbance. Note however that our magnitude of change is computed differently from those used in other studies (Devries, Verbesselt, et al. 2015; Hamunyela, Verbesselt \& Herold 2016). In our study area, forest disturbances are known to occur at small-scale and in gradual manner (Devries, Verbesselt, et al. 2015). They may therefore have small magnitude of changes. This may explain why the magnitude of change was not a strong predictor of forest disturbance.

Although some space-time features were less important than others, it is important to highlight here that the importance of each space-time feature as predictor for forest disturbance may vary from one area to another, depending on the nature of forest disturbance. In areas where the forest is cleared on large scale and involves sudden and full forest cover removal, the magnitude of change might be a strong predictor of forest disturbance. Nonetheless, the space-time feature approach is flexible because the probability of disturbance is 
calculated using a supervised machine learning algorithm (Breiman 2001). However, elaborate training data set to train the learning algorithm is needed to ensure the applicability of this approach to different forest areas. The requirement for training data is therefore disadvantageous because training data for forest disturbances are often lacking in many parts of the world (Cohen et al. 2010), especially for small-scale forest disturbances which are difficult to acquire through visual interpretation of satellite images. Nonetheless, with advent of community-based forest monitoring (Pratihast et al. 2012; Pratihast et al. 2016), observations on forest disturbances acquired through citizen science and local experts could be used for training purposes (See et al. 2014; Pratihast et al. 2016; Pratihast et al. 2014; Devries et al. 2016). Availability of daily high-resolution image from nanosatellites sensors (e.g. Planet's satellites) may also improve acquisition of training data.

\subsubsection{Space-time features approach in the era of multi-sensor forest disturbances}

The approach of using space-time features we presented in this chapter is generic because the features we proposed here can be computed from image time series of any satellite sensors (e.g. Sentinel-2), including Synthetic Aperture Radar (SAR) sensors whose data are widely used for forest monitoring in recent years (Lehmann et al. 2012; Lehmann et al. 2015; Reiche et al. n.d.; Reiche, de Bruin, et al. 2015; Reiche, Verbesselt, et al. 2015). Our approach can also be applied to other optical satellite data metrics. We can for example use of all spectral bands, and extract space-time features from each spectral band. Such multi-spectral space-time features can then be used to predict the probability of forest disturbances. In this way, we can concurrently exploit spatial, temporal and spectral information in satellite data to accurately and timely map forest disturbance. It should be noted however that a clear understanding of how reflectance in each spectral channel reacts to forest disturbance is needed to extract consistent and meaningful space-time features.

\subsection{Conclusion}

This chapter demonstrated that using space-time features extracted from Landsat NDVI data cubes to confirm forest disturbance increases the capacity to reject many false detections, without compromising the temporal accuracy. The use of space-time features is based on the idea that forest disturbances, by default, are spatio-temporal processes, hence information on spatio-temporal association and variability in satellite time series should be taken in account to accurately and timely detect forest disturbances. The following space-time 
features were the strongest predictors for forest disturbance: i) the change in spatio-temporal variability, ii) spatio-temporal association with non-forest, and iii) variability in the reference period. Magnitude of change and two consecutive negative anomalies, which are widely used to distinguish real changes from false detections, were not main predictors of forest disturbance. These findings indicate that space-time features have potential to improve the detection forest disturbances, especially small-scale and gradual forest disturbances whose magnitude of change is often small. Detection of small scale forest disturbances may further improve when using satellite sensors, like Sentinel-2, that provides increased spatial and temporal details.

\section{Acknowledgements}

The work was funded by the European Space Agency (ESA) ForMoSa project Forest Degradation Monitoring with Satellite Data Project (grant agreement 5160957022), and the European Commission Horizon 2020 BACI project (grant agreement 640176). 



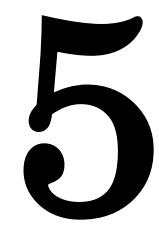

\section{Combining Sentinel-2 and Landsat time series for small-scale forest change monitoring}

This chapter is based on:

Hamunyela, E., Reiche, J., Verbesselt, J., Tsendbazar, N.E., \& Herold, M. Combining Sentinel-2 and Landsat time series for small-scale forest change monitoring, in prep. 


\section{Abstract}

Quantifying forest loss accurately and timely is important for initiatives that aim to reduce carbon emissions from deforestation and forest degradation. Yet, in many parts of the globe, quantifying forest loss accurately and timely is often constrained by our limited capacity to map small-scale forest disturbances. Here, we evaluate whether mapping of small-scale forest disturbances can be improved by detecting forest disturbances using the $10 \mathrm{~m}$ resolution Sentinel-2 time series. To do this, we compared area estimates for forest disturbance assessed from Sentinel-2 time series at $10 \mathrm{~m}$ and $30 \mathrm{~m}$ resolutions. We also evaluated how observation density in the time series affects the detection of small-scale forest disturbances at $30 \mathrm{~m}$ resolution by detecting forest disturbances at $30 \mathrm{~m}$ resolution using a combined time series of all available Landsat-7/ETM+, Landsat-8/OLI and Sentinel-2/MSI observations. We detected forest disturbances in UNESCO Kafa Biosphere Reserve, southwest of Ethiopia, where small-scale forest disturbances are common, using normalised vegetation index (NDVI) time series. NDVI time series was normalised spatially to reduce seasonality and inter-sensor differences. Landsat time series (2013 -2015) was used as a historical reference. We show that spatial normalisation reduces intersensor differences in Landsat-Sentinel-2 NDVI time series significantly, resulting in temporally consistent multi-sensor time series. Our results show that, detecting forest disturbances at $10 \mathrm{~m}$ Sentinel-2 resolution improves the detection of small-scale forest disturbances significantly. At the crossover point, area estimate for disturbed forest derived from Sentinel-2 10m resolution was $2115.9 \pm 65.4$ ha, whereas the area estimate derived from Sentinel-2 30m resolution was $1397.9 \pm 40.5$ ha. Sentinel-2 $10 \mathrm{~m}$ resolution allowed for detection of small-scale disturbances (e.g. road networks), which could not be detected using the $30 \mathrm{~m}$ resolution, but it did not supersede the importance of monitoring forest disturbances using dense time series. The area estimate from $10 \mathrm{~m}$ resolution was not significantly different from those estimated from the $30 \mathrm{~m}$ resolution scenario $(2245.1 \pm 68.6 \mathrm{ha})$ when using a combined time series of all available Landsat and Sentinel-2 observations. With a Sentinel-2B/MSI launched successfully, free and open access dense time series at $10 \mathrm{~m}$ resolution will become available, and will further improve our capacity to map small-scale forest disturbances.

Keywords: Sentinel-2, Landsat, Data cubes, Change detection, Small-scale, Forest disturbance 


\section{$5.1 \quad$ Introduction}

Quantifying forest loss accurately and timely is important for initiatives that aim to reduce carbon emissions from deforestation and forest degradation (Ryan et al. 2012; Anderson et al. 2005; Diniz et al. 2015; Hansen et al. 2016; Achard et al. 2007; Hansen et al. 2010). However, in many parts of the globe, quantifying forest loss accurately and timely is often constrained by our limited capacity to map small-scale forest disturbances (Tyukavina et al. 2013). Over the years, there has been effort to improve the detection of small-scale forest disturbances by using all available medium resolution $(30 \mathrm{~m})$ Landsat time series (Devries, Verbesselt, et al. 2015; Zhu et al. 2012; Hamunyela, Verbesselt \& Herold 2016; Hansen et al. 2016), but our capacity to map small-scales forest disturbances (<10ha) remains limited (Hansen et al. 2016).

Several factors limit our capacity to map small-scales forest disturbances using Landsat time series. Firstly, in tropical regions, availability of valid Landsat observations per year is generally limited due to persistent cloud cover (Asner 2001). This low observation density affects the detection of forest disturbances from Landsat time series (Reiche, Verbesselt, et al. 2015; Reiche, de Bruin, et al. 2015). Fortunately, with advent of free and open access SAR observations from Sentinel-1 sensors, low observation density in the tropics can now be addressed (Reiche et al. in review). Secondly, the choice of the change detection algorithm can also limit the detection of small-scale forest disturbances. Some algorithms are tuned to reduce omission error, other are tuned minimise the commission error (Cohen et al. 2017). Detecting forest disturbances using change detection algorithms tuned for higher-magnitude disturbances reduces the capacity to detect subtle forest disturbances (Cohen et al. 2017). Algorithms that rely on magnitude of change to balance between the commission and omission error (e.g.Devries et al., 2015) can also increase the omission of small-scale disturbances. Thirdly, spatial resolution of Landsat can also affect our capacity to detect small-scale forest disturbances. In many parts of the globe, human-induced forest disturbances occur at small spatial scale (Devries, Verbesselt, et al. 2015; Ryan et al. 2012; Fisher 2010), and majority of such disturbances may be too small to capture using the medium resolution Landsat data. For example, a logging road may be too narrow to capture using Landsat data. Disturbances caused by small-holder agriculture expansion, domestic firewood and charcoal extractions may also be too small to detect correctly using Landsat time series. Satellite sensors such as RapidEye $(5 \mathrm{~m})$ and PlanetScopes $(3.7 \mathrm{~m})$ provide high spatial resolution data which can enhance the detection of small-scale forest disturbances, but these high resolution satellite data are distributed on commercial basis. However, procuring long time series data acquired by commercial satellites can be costly (Wulder \& Coops 2013), thus limiting the application of such data for frequent and large-area forest change monitoring (Wulder et al. 2012; Reiche et al. 2016). 
With the advent of two Sentinel-2 Multi-Spectral Instrument (MSI) satellites, free and open access satellite data with much higher spatial resolution $(10 \mathrm{~m})$ than Landsat are now available. However, it is not yet known whether using the $10 \mathrm{~m}$ resolution Sentinel- 2 time series improves the detection of small-scale forest disturbances. This chapter aims to evaluate the potential of the $10 \mathrm{~m}$ resolution Sentinel-2 time series for small-scale forest change monitoring. The first Sentinel-2 sensor (Sentinel-2A/MSI) was launched on 23 June 2015, and the second sensor (Sentinel-2B/MSI) was only launched recently (7 March 2017). Therefore, Sentinel-2 time series currently lacks sufficient historical observations. Yet, sufficient historical observations are required for robust forest change detection. Landsat has sufficient historical observations, and could potentially be used as reference for Sentinel-2 time series, but combining time series from multiple sensors is often challenging because of the intrinsic inter-sensor differences. Apart from spatial resolution, earth observing satellites often have different radiometric calibrations, viewing angles, orbit times, and spectral resolutions. These inter-sensor differences can propagate disjoints in multi-sensor time series, resulting in many false abrupt changes (Tian et al. 2015).

Although Landsat and Sentinel-2 sensors are considerably similar (Figure 5.1), they also have substantial differences (Mandanici \& Bitelli 2016) in their sensor parameters. For example, Sentinel-2/MSI has radiometric quantization (12 bit) similar to Landsat-8/OLI, but different from Landsat-7/ETM+ (8 bit). Sensors with high radiometric quantization are more sensitive to changes in reflectance signal. The spectral resolution for Sentinel-2/MSI in near-infrared (NIR) region $(785-900 \mathrm{~nm})$ is also narrower than that of Landsat 7/ETM+ (775 $900 \mathrm{~nm})$, but wider than that of Landsat-8/OLI $(845-885 \mathrm{~nm})$. The difference in spectral resolution propagates considerable inter-sensor differences between multi-sensor data. For example, recent studies show that, differences in the NIR spectral resolution between the Landsat-7/ETM+ and Landsat-8/OLI sensors were clearly pronounced in the normalised difference vegetation index (NDVI) values (Roy et al. 2016; Li et al. 2014). Another difference between Landsat 7/ETM+, Landsat-8/OLI and Sentinel-2/MSI is the spatial resolution. Sentinel$2 / \mathrm{MSI}$ acquires data at $10 \mathrm{~m}$ resolution in the visible and NIR regions only, other spectral regions are acquired either at $20 \mathrm{~m}$ or $60 \mathrm{~m}$ resolution, thus limiting the integration of Sentinel-2 and Landsat data for forest change monitoring mainly to the visible and NIR channels.

To combine Landsat and Sentinel-2 time series, their differences in spectral resolutions should be reduced to ensure that such differences do not propagate artificial abrupt changes or increase the variance in a Landsat-Sentinel-2 time series. Traditionally, inter-sensors differences in multi-sensor time series are reduced using adjustment factors (Roy et al. 2016; Steven et al. 2003). Adjustment factors are derived by using the configurations or characteristics of one sensor as a reference for other sensors (Fan \& Liu 2017). Although this 
approach works (Roy et al. 2016), it has serious limitations because an undesirable change in the condition of the reference sensor would render prior adjustment factors invalid. Correcting inter-sensor differences in multi-sensor time series using adjustment factors is nonetheless essential for quantitative analysis (e.g. vegetation trend analysis) to maintain the quantitative meaning of the observations. For forest cover change monitoring, however, maintaining the quantitative meaning of the observations is not important. What is important is to ensure that the impact of forest disturbances in the time series is preserved when reducing inter-sensor differences.

For forest change monitoring, inter-sensor differences in multi-sensor time series can potently be reduced through spatial normalisation. Recently, spatial normalisation has been used to reduce seasonal variations in satellite image time series (Hamunyela, Verbesselt, Bruin, \& Herold, 2016; Hamunyela, Verbesselt, \& Herold, 2016; Reiche, Hamunyela, Verbesselt, Hoekman, \& Herold, in review), but its suitability to reduce inter-sensor differences is not known. With spatial normalisation, inter-sensor differences in multi-sensor time series would be reduced by normalising each pixel value in each image spatially using information derived from the neighbouring pixels.

The objectives of this chapter are to: (i) assess whether monitoring forest disturbances at $10 \mathrm{~m}$ resolution leads to more accurate forest change detection than when using $30 \mathrm{~m}$ resolution, (ii) investigate whether Landsat and Sentinel-2 observations harmonization improves the detection of small-scale forest disturbances compared to Landsat only scenario, (iii) evaluate whether spatial normalisation can reduce inter-sensor differences in Landsat-Sentinel-2 time series. We hypothesised that normalising Landsat-Sentinel-2 time series spatially would reduce inter-sensor differences significantly, resulting in a temporally consistent multi-sensor time series suitable for forest change detection. We also assumed that monitoring forest disturbance at $10 \mathrm{~m}$ resolution would lead to more accurate detection of small-scale forest disturbances than when using the $30 \mathrm{~m}$ resolution. 


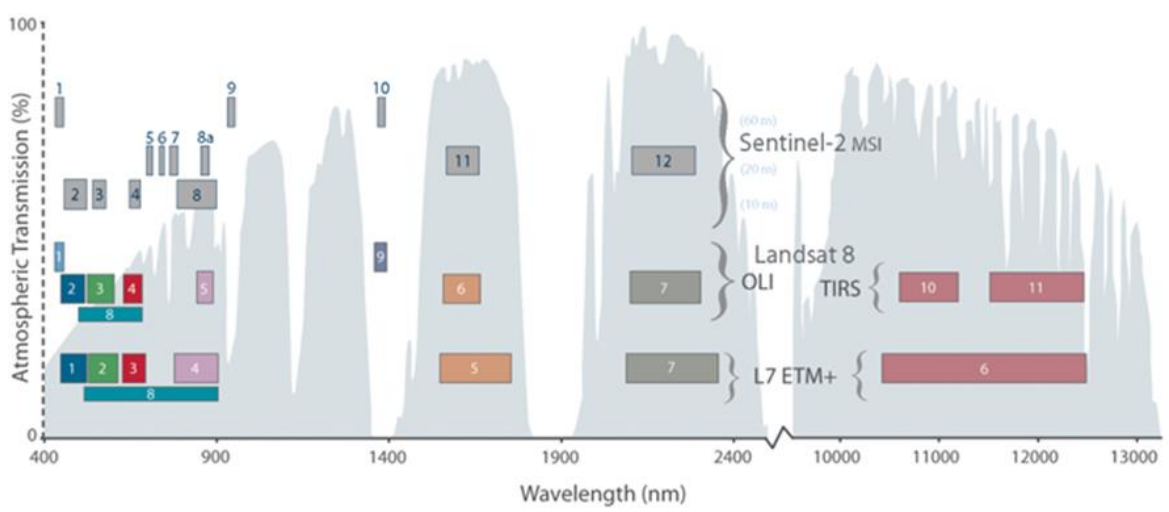

Figure 5.1: Spectral comparison between Landsat 7/ETM+, Landsat-8/OLI and Sentinel-2/MSI (source: https://landsat.gsfc.nasa.gov/sentinel-2alaunches-our-compliments-our-complements/).

\section{$5.2 \quad$ Study area}

Our analysis focused on detecting forest disturbances in the UNESCO Kafa Biosphere Reserve, southwest Ethiopia (Fig. 1), where small-scale forest disturbances are known be common (Dresen et al. 2014; Pratihast et al. 2014). The forest in Kafa is a moist Afromontane broadleaf evergreen forest that exhibits a moderate seasonality. We chose Kafa Biosphere Reserve particularly for two reasons. First, it is part of the areas where Sentinel-2/MSI has consistently been acquiring data since its launch, thus allowing us to evaluate the importance of integrating optical data streams from Landsat and Sentinel-2 sensors for forest monitoring. Second, forest disturbances in Kafa Biosphere Reserve occur at small spatial scale and are generally gradual (DeVries et al., 2015). Such small-scale and gradual change processes are difficult to detect. This study area is therefore particularly ideal for evaluating whether improved spatial resolution of Sentinl-2 improves the detection of small-scale forest disturbances when compared to $30 \mathrm{~m}$ resolution.

Kafa Biosphere Reserve is over 700,000 ha in size and is one of the few remaining natural habitats for Coffee Arabica (Aerts et al. 2015), and of many other endemic species of plants, mammals and birds (Schmitt et al. 2010). Yet, forests in this area are subjected to human-induced disturbances mainly caused by small-holder agriculture, human settlements expansion, industrial coffee plantations, and domestic firewood and charcoal extractions (Dresen et al. 2014; Pratihast et al. 2014). Recent studies (Pratihast et al. 2016; Devries, Verbesselt, et al. 2015; Devries et al. 2016) show that small-scale forest disturbances continue to occur in Kafa Biosphere Reserve. 


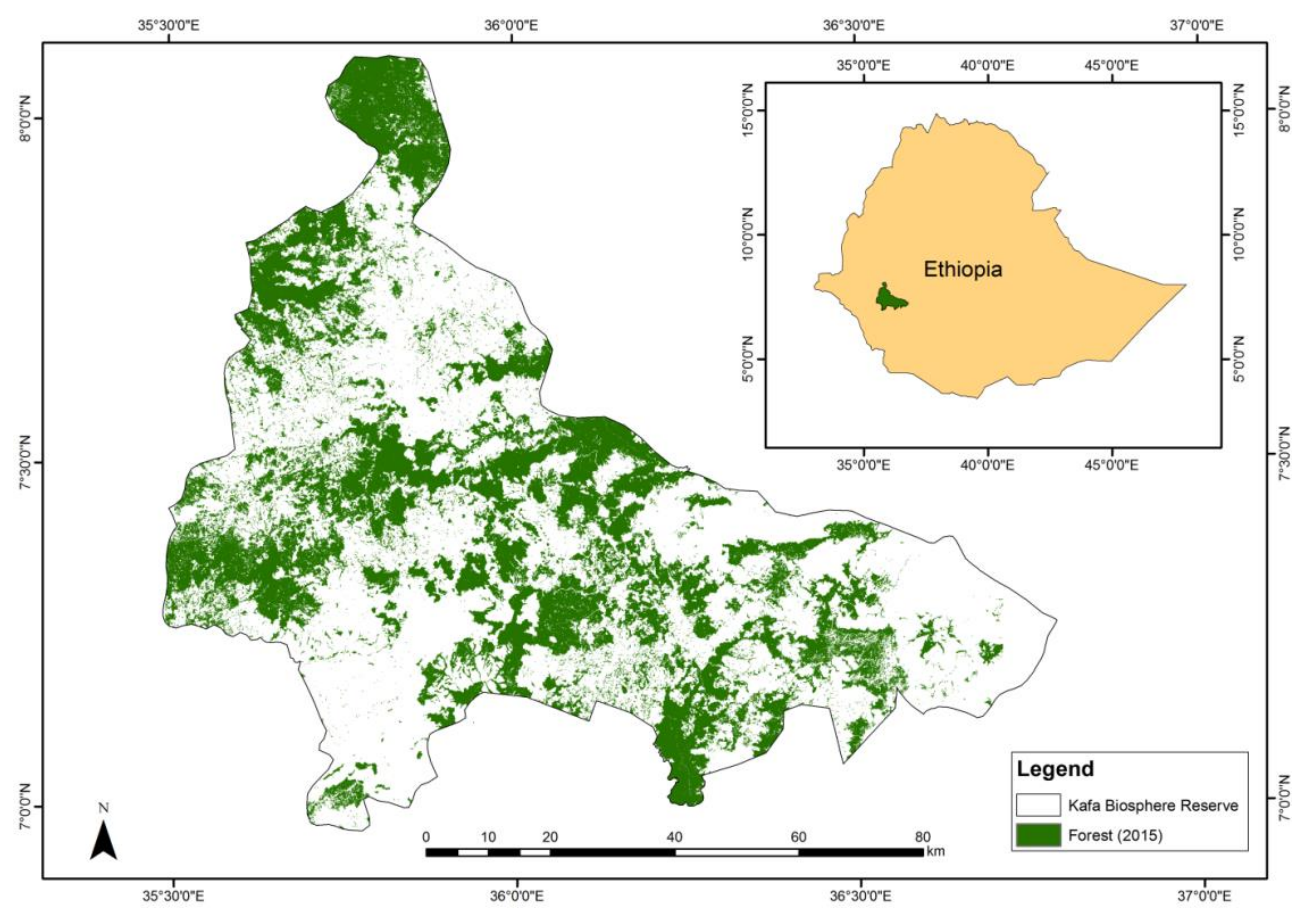

Figure 5.2: Overview of the UNESCO Kafa Biosphere Reserve located in southwestern Ethiopia, The green areas represented the forest cover in Kafa Biosphere Reserve in year 2015. The forest areas were identified using supervised maximum likelihood classifier.

\subsection{Data and methods}

An overview of the methods we followed in this study to detect forest disturbances from Landsat-Sentinel-2 time series is shown in Figure 5.2. First, we masked cloud and cloud shadows in Landsat (Section 5.3.1.1) and Sentinel-2 (Section 5.3.1.2) multi-spectral images. Second, Landsat multi-spectral images were co-registered to Sentinel-2 multi-spectral images (Section 5.3.1.3). We derived NDVI (Rouse, Haas, Scheel, \& Deering, 1974; Tucker, 1979) from coregistered Landsat-7/ETM+, Landsat-8/OLI and Sentinel-2/MSI images. Third, we produced a benchmark forest mask (Section 5.3.1.4), and use it to mask nonforest areas in the Landsat and Sentinel-2 time series. Fourth, we combined Landsat and Sentinel-2 NDVI time series at $30 \mathrm{~m}$ and $10 \mathrm{~m}$ resolutions (Section 5.3.1.5). Firth, Landsat and Sentinel-2 NDVI were normalised spatially to reduce seasonality and inter-sensor differences in the time series (Section 5.3.1.6). Sixth, we quantified inter-sensor differences in Landsat-Sentinel-2 NDVI pixel time series before and after spatial normalisation (Section 5.3.2). Seventh, in Section 5.3.3, we detected forest disturbances in Sentinel-2/MSI NDVI time 
series at $10 \mathrm{~m}$ and $30 \mathrm{~m}$ resolution while using observations from LandsatSentinel-2 (2013 -2015) as a historical reference to assess the effect of spatial resolution on the detection of forest disturbances in Kafa Biosphere Reserve. For the $10 \mathrm{~m}$ scenario, Landsat time series were disaggregated to $10 \mathrm{~m}$ resolution. Forest disturbances were also detected in Landsat time series only, and in a combined time series of all available Landsat and Sentinel-2 at $30 \mathrm{~m}$ to assess the effect of observation density for detection of forest disturbances in Kafa Biosphere Reserve. Finally, spatial and temporal accuracy, as well as and forest loss estimates were calculated using a sample-based reference data (Section 5.3.4).

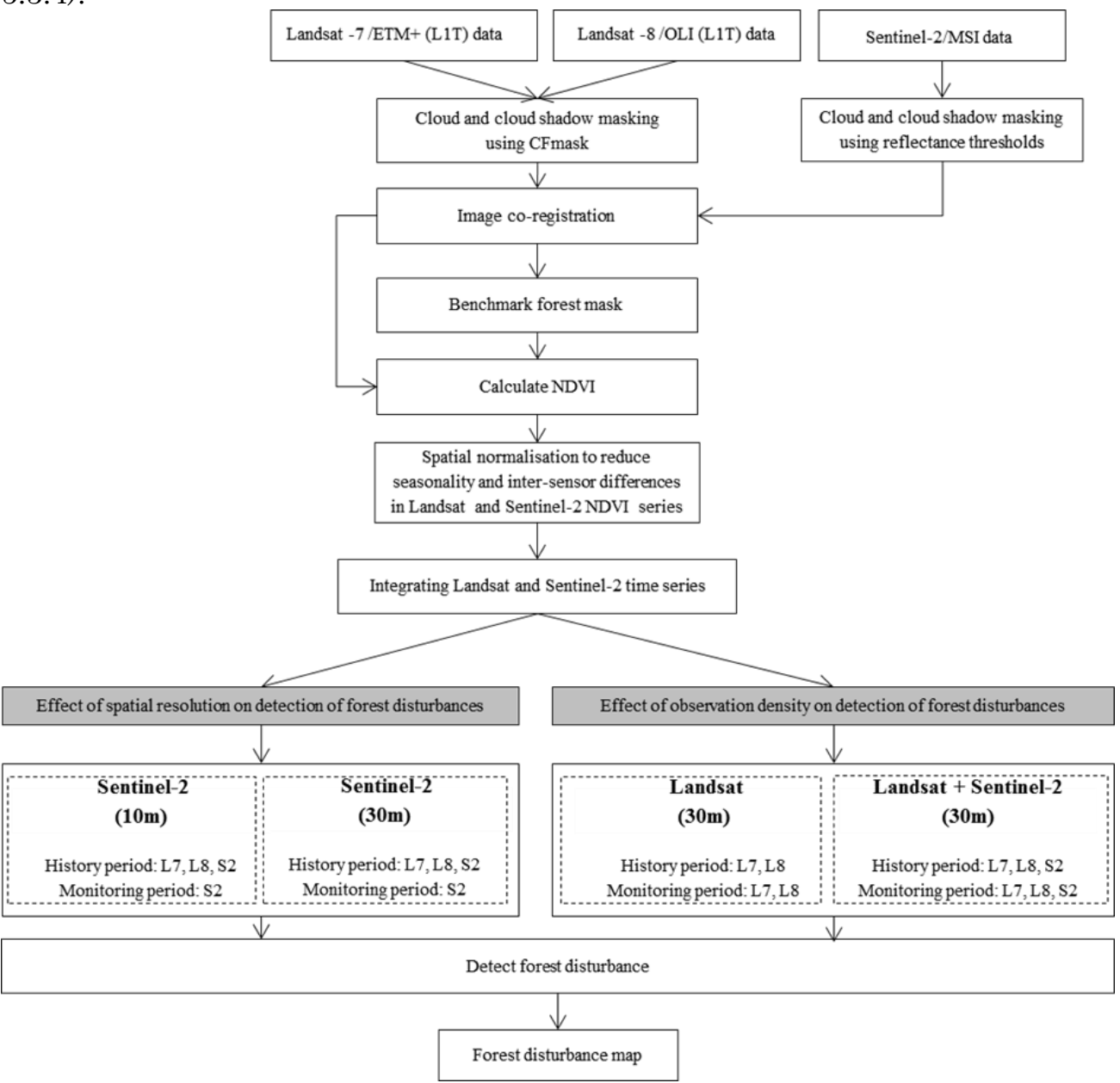

Figure 5.3: The workflow for detecting forest disturbances using LandsatSentinel-2 image time series. 
Table 5.1: Number of NDVI images from Landsat-7/ETM+, Landsat-8/ OLI and Sentinel-2/ MSI sensor that were used for detection of forest cover loss UNESCO Kafa Biosphere Reserve.

\begin{tabular}{lccc}
\hline Sensor & $\begin{array}{c}\text { No. of images in } \\
\text { history period }\end{array}$ & $\begin{array}{c}\text { No. of images in } \\
\text { monitoring period }\end{array}$ & $\begin{array}{c}\text { Total no. of } \\
\text { images }\end{array}$ \\
\hline Landsat-7/ETM+ & 55 & 16 & 71 \\
Landsat-8/OLI & 46 & 16 & 62 \\
Sentinel-2/MSI & 3 & 18 & 21 \\
\hline
\end{tabular}

\subsubsection{Pre-processing of satellite data}

\subsubsection{Landsat data}

We sourced geometrically and atmospherically corrected Landsat-7/ETM+ and Landsat-8/ OLI surface reflectance products from The United State of America's Geological Survey (USGS) Landsat surface Reflectance (SR) Climate Data Records (CDR; http://landsat.usgs.gov/CDR_LSR.php). Landsat-7/ETM+ surface reflectance products were generated using Landsat Ecosystem Disturbance Adaptive Processing System (LEDAPS) algorithm (Masek et al. 2006), whereas Landsat 8 OLI surface reflectance products are generated using Landsat 8 OLI surface reflectance algorithm (Vermote et al. 2016). We masked the clouds and cloud shadows in Landsat-7/ETM+ and Landsat-8/OLI images using the CFmask cloud-shadow mask product (Zhu \& Woodcock 2012) which is distributed together with the Landsat SR CDR Products.

\subsubsection{Sentinel-2 data}

We used Sentinel-2 data acquired by Sentinel-2A/MSI between November 2015 and December 2016. Similar to Pipitone et al., (2016), we generated the Sentinel-2 bottom of atmosphere (BOA) reflectance products from the top of atmosphere (TOA) Level-1C data using Sen2cor software (Uwe et al. 2013). TOA reflectance data were sourced from the Sentinels Scientific Data Hub (https://scihub.copernicus.eu). Currently, a standard algorithm for masking clouds and cloud shadows in Sentinel-2/MSI data does not exist. To mask clouds in Sentinel-2/MSI data, we used reflectance thresholds derived from coastal aerosol band (Band 1, disaggregated to $10 \mathrm{~m}$ resolution) and blue band (Band 2). For shadow masking, we used reflectance thresholds derived from near-infrared band (Band 8) and short-wave infrared band (Band 12, disaggregated to $10 \mathrm{~m}$ resolution). Thresholds and the procedure we used to detect and mask clouds and cloud shadows in Sentinel-2 multi-spectral images are shown in Table 5.2. Note that the thresholds were derived from images whose reflectance values 
were scaled by 10000 . Reflectance thresholds were derived by training a logistic regression to determine the probability of a pixel being clear, contaminated by a cloud or cloud shadow given its reflectance values. In the next step, we calculated classification accuracy for each class, while varying the probability for clouds or shadows. We chose the reflectance thresholds that achieved the highest classification accuracy for clouds and shadow. We trained the logistic regression using data manually digitised from Sentinel-2/MSI data acquired on 29-11-2015 and 08-03-2016. Thresholds for cloud and cloud shadows were validated using a test dataset also manually digitised from Sentinel-2/MSI data, acquired on 1702-2016 and 29-12-2015. Reflectance thresholds, derived from the bands we used here, have been used recently to detect and mask clouds and cloud shadows in Sentinel-2 multi-spectral images (Verhegghen et al. 2016).

Table 5.2: Cloud and cloud-shadow thresholds and a procedure for detecting and masking clouds and cloud shadows in Sentinel-2 multi-spectral images. Note that the thresholds were derived from images whose reflectance values were scaled by 10000 .

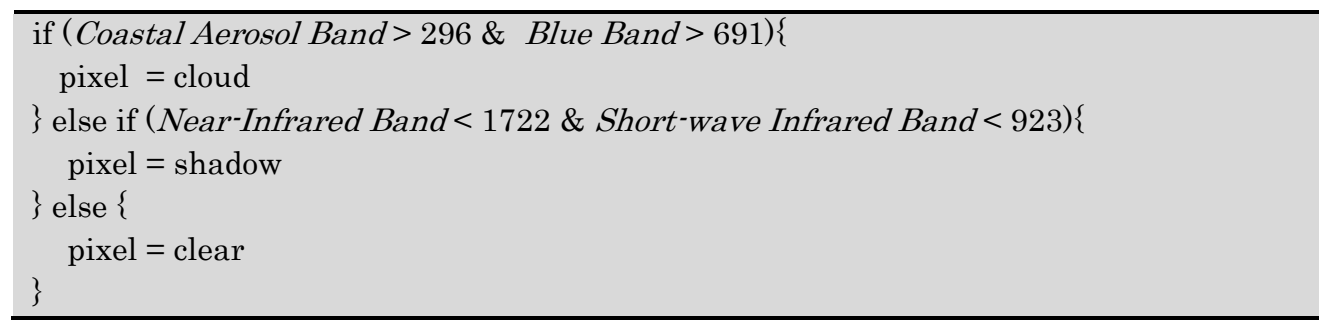



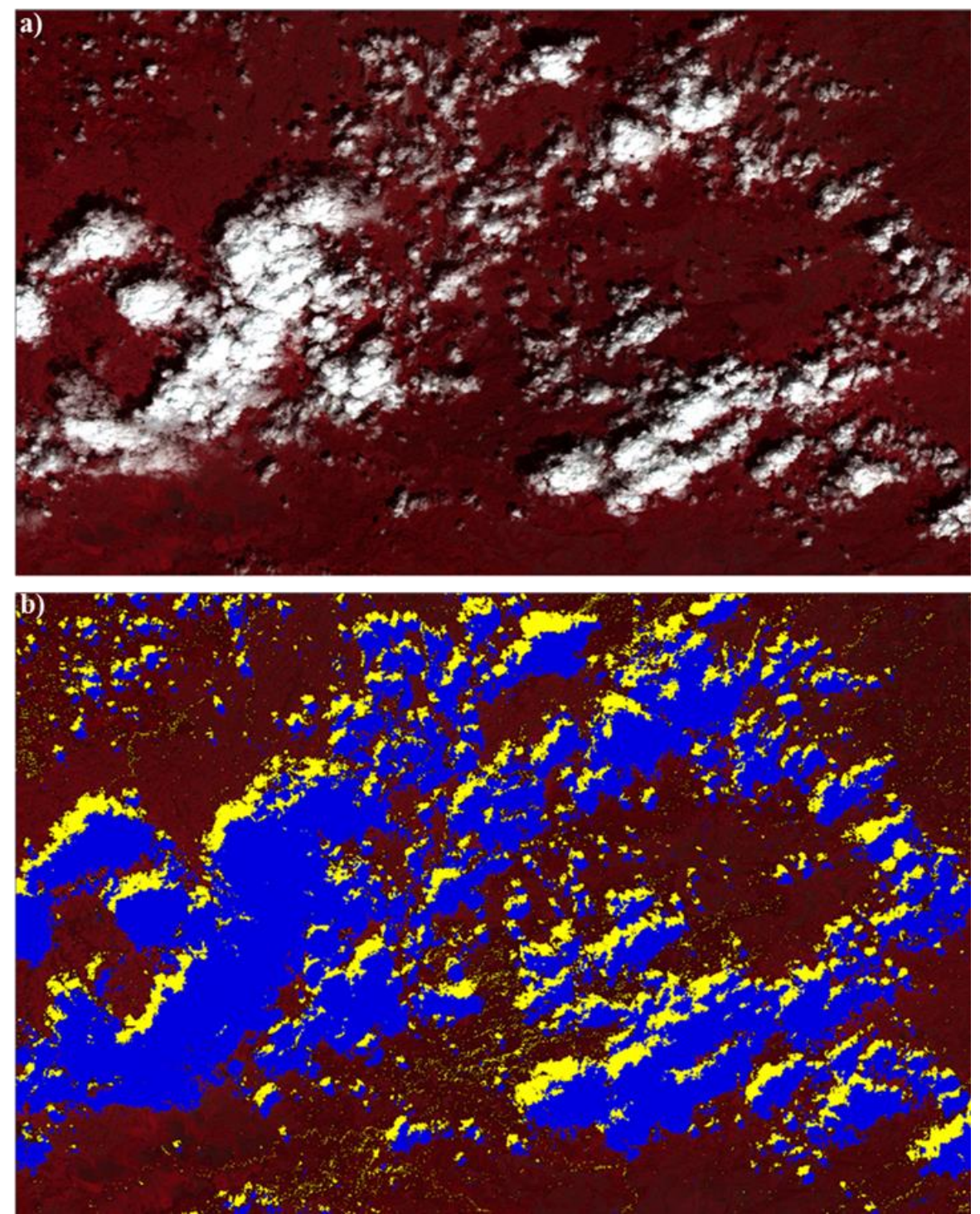

Figure 5.4: An example of how cloud and cloud shadows were identified in Sentinel-2/MSI image using our thresholds. The blue colour in (b) represents areas in (a) which were identified as clouds, and the yellow colour in (b) represents areas identified as shadows. The base image is band 8-3-2 composite, which is part of a Sentinel-2/MSI image acquired over UNESCO Kafa Biosphere Reserve, southwest of Ethiopia, in 2015-11-29. 


\subsubsection{Co-registration of Landsat and Sentinel-2 images}

Accurate image-to-image co-registration is a prerequisite for meaningful time series-based change detection. Currently, however, there is a known sensorto-sensor geolocation error of about $38 \mathrm{~m}$ between Landsat-8/OLI and Sentinel2/MSI (Storey et al. 2017). Here, we co-registered Landsat and Sentinel-2 images using the GAMMA software package (Werner \& Strozzi 2000) to ensure good image-to-image co-registration. Landsat multi-spectral images were coregistered to Sentinel-2 multi-spectral images. Co-registration steps involved an automated cross-correlation between the source image (Landsat) and a reference target image (Sentinel-2) using the least-squares polynomial fitting, which resulted in range and azimuth offset of $4^{\text {th }}$ polynomial order. The offset polynomials were then used to co-register the source image to the reference target image achieving a sub-pixel accuracy smaller than 0.01 pixel.

\subsubsection{Benchmark forest mask}

To avoid detecting changes occurring in other land cover types than forest loss, we masked non-forest areas using a benchmark forest mask. The benchmark forest mask was generated from Landsat-8/OLI multi-spectral image time series for 2015 using a supervised maximum likelihood classifier. Forest patches smaller than 0.54 ha were excluded in order to avoid including transitional mosaic areas composed of trees planted around the crop fields. Such transitional mosaic areas are common in UNESCO Kafa Biosphere Reserve and can amplify commission error if not excluded (Devries, Verbesselt, et al. 2015). In total, the final forest mask product contained 2985996 forest pixels $(\approx 268740$ ha).

\subsubsection{Spatial normalization to reduce seasonality and inter-sensor differences in the time series}

Accounting for seasonality in satellite image time series is essential for robust forest cover change detection. The moist Afromontane broadleaf evergreen forest in the UNESCO Kafa Biosphere Reserve exhibits moderate seasonality (Devries, Verbesselt, et al. 2015). In addition, differences in spectral resolution (NIR channel) propagate significant inter-sensor differences in NDVI time series from multiple sensors (Ke, Im, Lee, Gong, \& Ryu, 2015; Roy et al., 2016). If not accounted for, both forest seasonality and inter-sensor differences can affect the detection of forest disturbances adversely, either by increasing commission error or increasing the omission error because of high variance in the time series. Here, we used spatial normalisation (Hamunyela, Verbesselt \& Herold 2016) to reduce seasonality and inter-sensor differences in LandsatSentinel-2 NDVI time series. Seasonality and inter-sensor differences are reduced by dividing the NDVI value for each pixel in each image with a global 
95 ${ }^{\text {th }}$ percentile $\left(\mathrm{P}_{95}\right)$ computed over the entire NDVI image. We used a global $\mathrm{P}_{95}$ for two main reasons. First, the forest in our study area was largely homogenous in terms of forest type. Second, unlike local $\mathrm{P}_{95}$, the global $\mathrm{P}_{95}$ eliminate the difficulty of selecting the suitable size for a local spatial window, and is more computationally efficient than a local spatial window.

\subsubsection{Combining Landsat and Sentinel-2 time series}

Among the 13 spectral regions for Sentinel-2/MSI, only the visible and NIR regions at $10 \mathrm{~m}$ resolution allow for straightforward spatial integration with Landsat data. Other spectral regions for Sentinel-2 sensors are either available at $20 \mathrm{~m}$ or $60 \mathrm{~m}$. Sentinel-2/MSI acquires data at $10 \mathrm{~m}$ resolution in the visible and NIR regions, whereas Landsat sensors acquire at $30 \mathrm{~m}$. To combine Landsat and Sentinel-2 time series, we first derived normalised difference vegetation index (NDVI) (Tucker 1979; Rouse et al. 1974) from pre-processed Landsat (30 m) and Sentinel-2 $(10 \mathrm{~m})$ data. Next, we combined Landsat and Sentinel-2 time series at two different spatial resolutions: $30 \mathrm{~m}$ and $10 \mathrm{~m}$. At the $30 \mathrm{~m}$, we first aggregated Sentinel- 2 time series to $30 \mathrm{~m}$ resolution, and combined all available Landsat and Sentinel-2 observations. At $10 \mathrm{~m}$ resolution, Landsat NDVI time series (2013 - 2015) disaggregated to $10 \mathrm{~m}$ resolution was combined with $10 \mathrm{~m}$ resolution Sentinel-2 NDVI time series.

\subsubsection{Quantifying inter-sensor differences in Landsat-Sentinel-2 pixel-time series}

To assess whether spatial normalisation can reduce inter-sensor differences in Landsat-Sentinel-2 NDVI time series, we quantified the differences before and after spatial normalisation. To quantify the inter-sensor differences between Landsat-7/ETM+, Landsat-8/OLI and Sentinel-2/MSI NDVI time series, we first calculated the median NDVI value from the time series for each sensor at each pixel. For each sensor, only observations acquired between November 2015 and December 2016 were included in the calculation of the median NDVI value. This period corresponds with the period when the Sentinel-2/MSI started acquiring data. We restricted the calculation of inter-sensor differences to the observations acquired between November 2015 and December 2016 to ensures that intersensor differences are calculated from data acquired during corresponding time of the year. After calculating the median NDVI value, we calculated the difference between the median NDVI values for two different sensors. For example, let ML7, ML8 and MS2 be the median values for Landsat-7/ETM+, Landsat-8/OLI and Sentinel-2/MSI NDVI time series at a particular pixel. Intersensor difference between Landsat-8/OLI and Landsat-7/ETM+ was quantified by subtracting ML7 from ML8; MS2 from ML8 for Landsat-8/OLI and Sentinel2/MSI differences; and MS2 from ML7 for Landsat-7/ETM+ and Sentinel-2/MSI 
differences. We used the median value because it is robust against outliers. After calculating the inter-sensor differences, we used a t-test to assess whether the median inter-sensor difference before spatial normalisation (BSN) was different from zero. Next, we used a paired t-test to assess whether spatial normalisation (ASN) reduces inter-sensor differences in NDVI time series significantly. We only compared inter-sensor differences for sample pixels $(n=399)$, based on the validation data, where no anomalies were detected (see Section 5.4.3) and where the time series for each sensor had at least more than six valid observations. A $5 \%$ significant level was used as threshold for accepting or rejecting the null hypothesis that the reduction in the inter-sensor differences after spatial normalisation is significant.

\subsubsection{Detecting forest disturbances from Landsat-Sentinel-2 time series}

We used a space-time change detection framework (Hamunyela, Reiche, Verbesselt, \& Herold, in review; Hamunyela, Verbesselt, Bruin, et al., 2016) that detects forest disturbances as extreme events in satellite data cubes to detect forest disturbances in Landsat- Sentinel-2 time series. With this change detection framework, potential forest disturbances in the satellite image time series are identified based on two consecutive negative anomalies (Hamunyela, Verbesselt, Bruin, et al. 2016). We used the $5^{\text {th }}$ percentile threshold computed from the observations available in the reference period of a local data cube to identify the negative anomalies. A $5^{\text {th }}$ percentile threshold was used because it leads to timely detection of forest disturbances (Hamunyela et al., in review; Hamunyela, Verbesselt, Bruin, et al., 2016). A local data cube is defined around each pixel, and it has both spatial and temporal extents. These extents are userdefined. A temporal extent corresponds to the length of the time series (2013 2016). Here, we used a spatial extent of 9 × 9 pixels. Once two consecutive negative anomalies are detected, a set of space-time features extracted from the local data cube is used to confirm forest disturbances(Hamunyela et al., in review). Forest disturbance is confirmed by first calculating the probability for forest disturbance with space-time features being predictors of forest disturbances. Forest disturbances are identified using a probability threshold, which is user-defined. The probability for forest disturbance was calculated using a trained random forest model $(n=2483$, trees $=1000 ;($ Breiman 2001) $)$.

\subsubsection{Estimating spatial and temporal accuracy, and area for disturbed forest}

For each monitoring scenario (Sentinel-2 at $10 \mathrm{~m}$, Sentinel-2 at $30 \mathrm{~m}$, Landsat at 30m , and Landsat + Sentinel-2 at 30m), we estimated area-adjusted spatial accuracy (overall accuracy, producer's and user's accuracy) and area estimates for forest cover disturbance and area bias (difference between commission error and omission error). Areas estimates and area bias were 
expressed in hectares. The accuracies and area of forest disturbances for maps with $30 \mathrm{~m}$ resolution (Sentinel-2 at $30 \mathrm{~m}$, Landsat at $30 \mathrm{~m}$, and Landsat + Sentinel-2 at 30m) were estimated using 2000 sample pixels (Change area $=500$, Buffer zone $=500$, No change area $=1000$ ) generated through stratified probability sampling (Stehman 2009) using the forest disturbance map (probability threshold $=0.5$ ) produced from Landsat + Sentinel-2 at $30 \mathrm{~m}$ scenario. The accuracies and area of disturbances for map with $10 \mathrm{~m}$ resolution (Sentinel-2 at 10m) were estimated also using 2000 sample pixels (Change area $=500$, Buffer zone $=500$, No change area $=1000)$ generated through stratified probability sampling (Stehman 2009). The stratification was based on the forest disturbance map (probability threshold $=0.5$ ) produced from Sentinel-2 at $10 \mathrm{~m}$ scenario.

To estimate overall accuracy, producer's and user's accuracy for rare class (disturbed forest) reliably, we allocated the sample size to each stratum based on a recommended approach (Stehman 2012; Olofsson et al. 2014). Based on this sample allocation, we calculated the area-adjusted spatial accuracy (overall accuracy, producer's accuracy and user's accuracy) and area estimates for forest disturbance. Apart from spatial accuracy and estimates, we calculated the temporal detection delay (expressed in days) for forest disturbances detected from Landsat at $30 \mathrm{~m}$ and Landsat + Sentinel-2 at $30 \mathrm{~m}$ to evaluate how observation density affects the temporal detection accuracy for small-scale forest disturbance. The temporal detection delay was defined as the number of days between the date of disturbance as per reference data and the acquisition date for the image in which a disturbance was confirmed.

Reference data were collected through visual interpretation of Landsat $(30 \mathrm{~m})$ and Sentinel-2 $(10 \mathrm{~m})$ multi-spectral time series. This approach of collecting reference data through visual interpretation of dense medium spatial resolution images is widely used in recent years (Devries, Verbesselt, et al. 2015; Hamunyela, Verbesselt \& Herold 2016; Zhu \& Woodcock 2014b; Dutrieux et al. 2015) because elaborate reference data for forest disturbance are scarce in many parts of the globe (Cohen, Yang, \& Kennedy, 2010). However, collecting reference data in this way has a major drawback, because forest disturbances which are smaller than the spatial resolution of the satellite images from which reference data are collected might not be visible, but could be detected, and might be interpreted incorrectly as commission error. 
Table 5.3: Area proportions, number of pixels and number of sample pixels per stratum based on forest disturbance map produced using the probability threshold of 0.5

\begin{tabular}{|c|c|c|c|}
\hline \multicolumn{4}{|c|}{$30 \mathrm{~m}$ resolution map } \\
\hline Stratum & No. of pixels & Area proportion & No. of sample pixels \\
\hline No change area & 2649105 & 0.887 & 2000 \\
\hline Buffer zone & 282173 & 0.095 & 500 \\
\hline Change area & 54230 & 0.018 & 500 \\
\hline Total & 2985508 & 1 & 2000 \\
\hline
\end{tabular}

\begin{tabular}{lccc}
\hline \multicolumn{4}{c}{10 m resolution map } \\
\hline Stratum & No. of pixels & Area proportion & No. of sample pixels \\
\hline No change area & 22335931 & 0.831 & 2000 \\
Buffer zone & 4118755 & 0.153 & 500 \\
Change area & 414886 & 0.016 & 500 \\
Total & 26869572 & 1 & 2000 \\
\hline
\end{tabular}

\section{$5.4 \quad$ Results}

\subsubsection{Effect of spatial normalisation on inter-sensor differences in Landsat- Sentinel-2 time series}

Our analysis show that statistically significant inter-sensor differences exist between the NDVI time series for Landsat-8/OLI and Landsat-7/ETM+ $(\mathrm{t}=$ $30.76, \mathrm{df}=398, \mathrm{p}<0.0001)$, Landsat-8/OLI, Sentinel-2/MSI $(t=19.37, \mathrm{df}=398, \mathrm{p}$ $<0.0001)$, and Landsat-7/ETM+ and Sentinel-2/MSI $(\mathrm{t}=-4.59$, df $=398, \mathrm{p}<$ 0.0001). However, these inter-sensor differences in Landsat-Sentinel-2 NDVI time series were reduced significantly by normalising the time series spatially. The median difference between Landsat-8/OLI and Landsat-7/ETM+ NDVI time series were reduced from 0.043 to $0.015(t=35.45,398, p<0.0001)$, from 0.030 to 0.020 for Landsat-8/OLI and Sentinel-2/MSI NDVI time series $(t=10.66, \mathrm{df}=$ $398, \mathrm{p}<0.0001$ ), and from 0.012 to 0.004 for Landsat-7/ETM+ and Sentinel2/MSI NDVI time series $(\mathrm{t}=-13.44$, $\mathrm{df}=398, \mathrm{p}<0.0001)$. Overall, the distribution of inter-differences becomes narrower after normalising the time series spatially (Figure 5.5). The inter-quartile range for Landsat-8/OLI Landsat-7/ETM+ differences, for example, decreased from 0.033 to 0.027 and from 0.033 to 0.027 for Landsat-8/OLI - Sentinel-2/MSI. Inter-quartile range for Landsat-7/ETM+ - Sentinel-2/MSI differences also decreased from 0.044 to 0.036. Based on the distributions of the differences, the NDVI for Landsat-8/OLI was higher than that of Landsat-7/ETM+ and Sentinel-2/MSI, whereas the NDVI for Landsat-7/ETM+ was lower than that of Sentinel-2/MSI. Fig. 5.6 show a multi- 
sensor time series for Landsat-8/OLI, Landsat-7/ETM+ and Sentinel-2/MSI before (BSN) and after (ASN) spatial normalisation. This example shows how the multi-sensor time series become more temporally consistent after spatial normalisation.
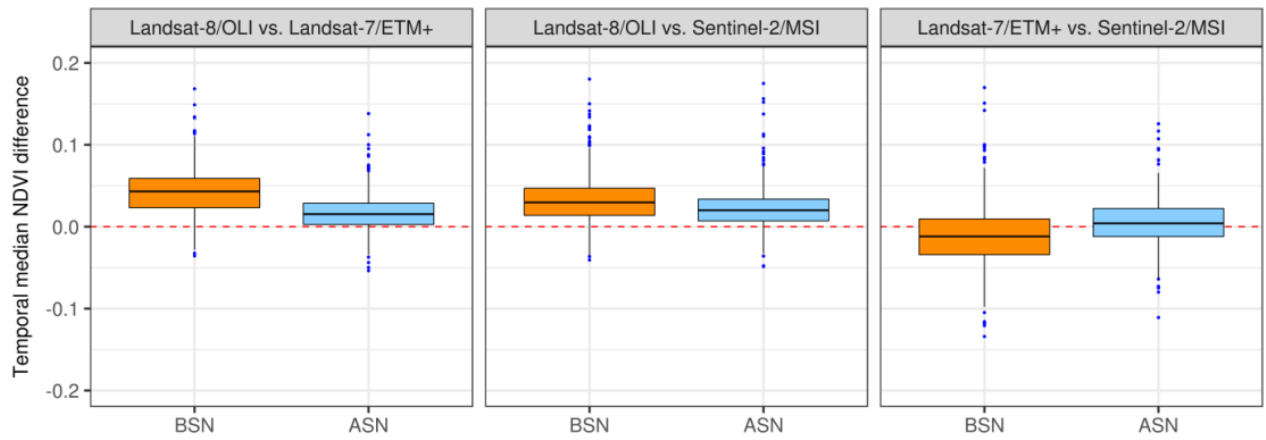

Figure 5.5: A boxplot for inter-sensor differences between the temporal median NDVI time series for Landsat-7/ETM+, Landsat-8/OLI and Sentinel-2/MSI before (BSN) and after (ASN) spatial normalisation

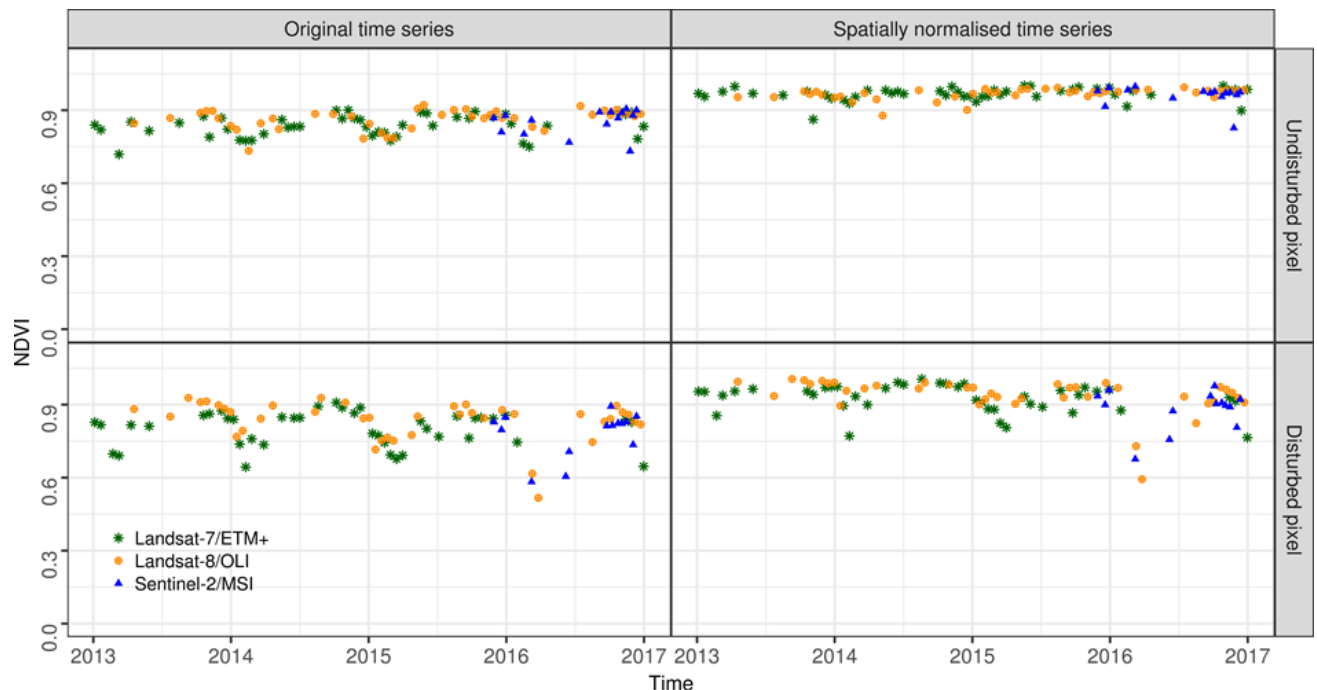

Figure 5.6: An example of a multi-sensor NDVI time series for Landsat-7/ETM+, Landsat-8/OLI and Sentinel-2/MSI before (BSN) and after (ASN) spatial normalisation. The time series for undisturbed forest cover is shown in (a), and (b) shows the time series for disturbed forest cover. 


\subsubsection{Effect of spatial resolution on detection of forest disturbances}

The omission error for forest disturbances was generally high both for the $10 \mathrm{~m}$ and $30 \mathrm{~m}$ resolution (Figure 5.7). At the probability threshold of 0 , the producer's accuracy for the $10 \mathrm{~m}$ resolution was only $54.6 \pm 11.7 \%$ (user's accuracy $=4.1 \pm 1.7 \%$ ), whereas the producer's accuracy for the $30 \mathrm{~m}$ resolution was $57.3 \pm 13.7 \%$ (user's accuracy $=3.3 \pm 1.6 \%$ ). At crossover point (lowest area bias), the producer's accuracy for the $10 \mathrm{~m}$ resolution was $50.7 \pm 3.1 \%$ (user's accuracy $=51.0 \pm 4.4 \%$ ), whereas the producer's accuracy for the $30 \mathrm{~m}$ resolution was $41.7 \pm 2.9 \%$. Note that the accuracies for $30 \mathrm{~m}$ and $10 \mathrm{~m}$ resolution are estimated using different reference datasets because of the different minimum mapping units. Detecting forest disturbances at $10 \mathrm{~m}$ resolution nonetheless led to more accurate area estimates for forest disturbance than when detecting forest disturbances at $30 \mathrm{~m}$ resolution (Figure 5.8). At the probability threshold of 0 , forest disturbances was estimated at $2280.7 \pm 266.2$ ha from $10 \mathrm{~m}$ resolution and at $1919.2 \pm 262.7$ ha from $30 \mathrm{~m}$ resolution. Estimated areas of forest disturbances decreased with increasing probability threshold for forest disturbance, reaching $166 \pm 2.7$ ha for $10 \mathrm{~m}$ scenario and $107.4 \pm 1.6$ ha for $30 \mathrm{~m}$ scenario at 0.95 probability threshold. Overall, the area bias for $10 \mathrm{~m}$ and $30 \mathrm{~m}$ resolutions was high at the low probability thresholds, but the area bias for $10 \mathrm{~m}$ resolution was generally lower than that of $30 \mathrm{~m}$ resolution. The area bias decreased exponentially with increasing probability threshold. For the $10 \mathrm{~m}$ resolution, the lowest area bias (-26.4 ha) was achieved at 0.5 probability threshold, whereas the lowest area bias $(-521.2 \mathrm{ha})$ for $30 \mathrm{~m}$ resolution was achieved at the probability threshold of 0.55 . The lowest area bias for $10 \mathrm{~m}$ and $30 \mathrm{~m}$ were all in favour of commission error, implying that the omission error was higher than the commission error. The area estimates at lowest bias was 2115.9 \pm 65.4 ha for the $10 \mathrm{~m}$ resolution and $1397.9 \pm 40.5$ ha for the $30 \mathrm{~m}$ resolution. Figure 5.9 shows an example of river channels which were omitted by the $30 \mathrm{~m}$ resolution but are clearly visible in $10 \mathrm{~m}$ resolution scenario. In our case, however, detection of these river channels is accounted as a commission error. 


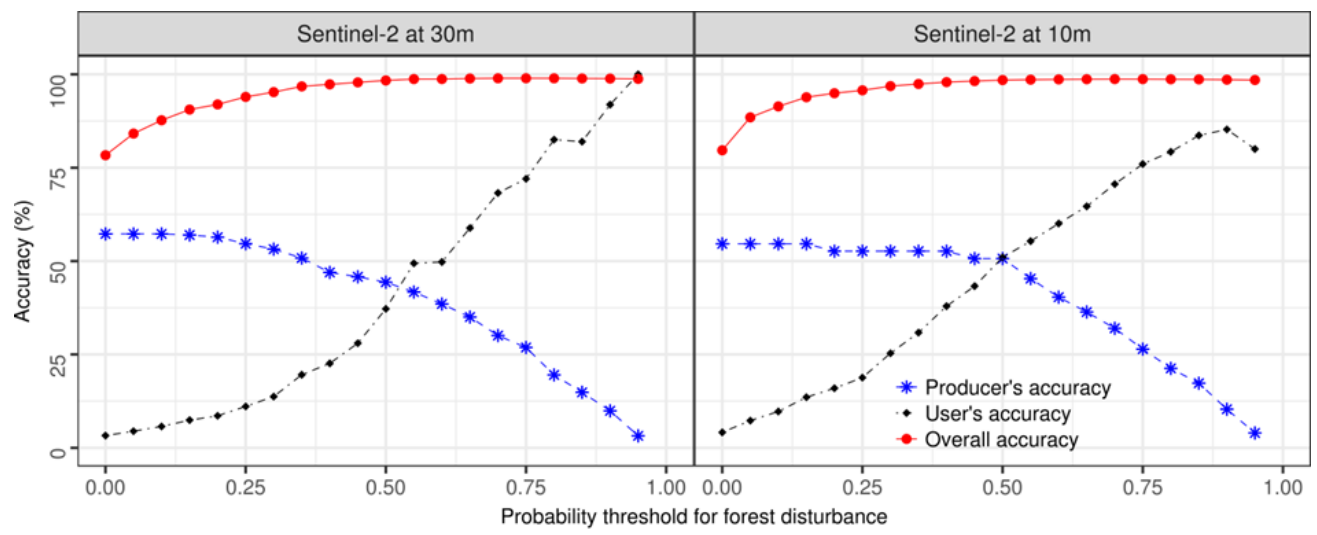

Figure 5.7: Overall accuracy, user's accuracy and producer's for forest disturbances detected in UNESCO Kafa Biosphere Reserve, southwestern Ethiopia in 2016 using Sentinel-2 normalised difference vegetation index (NDVI) time series at $30 \mathrm{~m}$ and $10 \mathrm{~m}$ resolution. Landsat and Sentinel-2 NDVI time series for $2010-2015$ was used as historical reference. Note that the accuracies for $30 \mathrm{~m}$ and $10 \mathrm{~m}$ resolution are estimated using different reference datasets because of the different minimum mapping units.
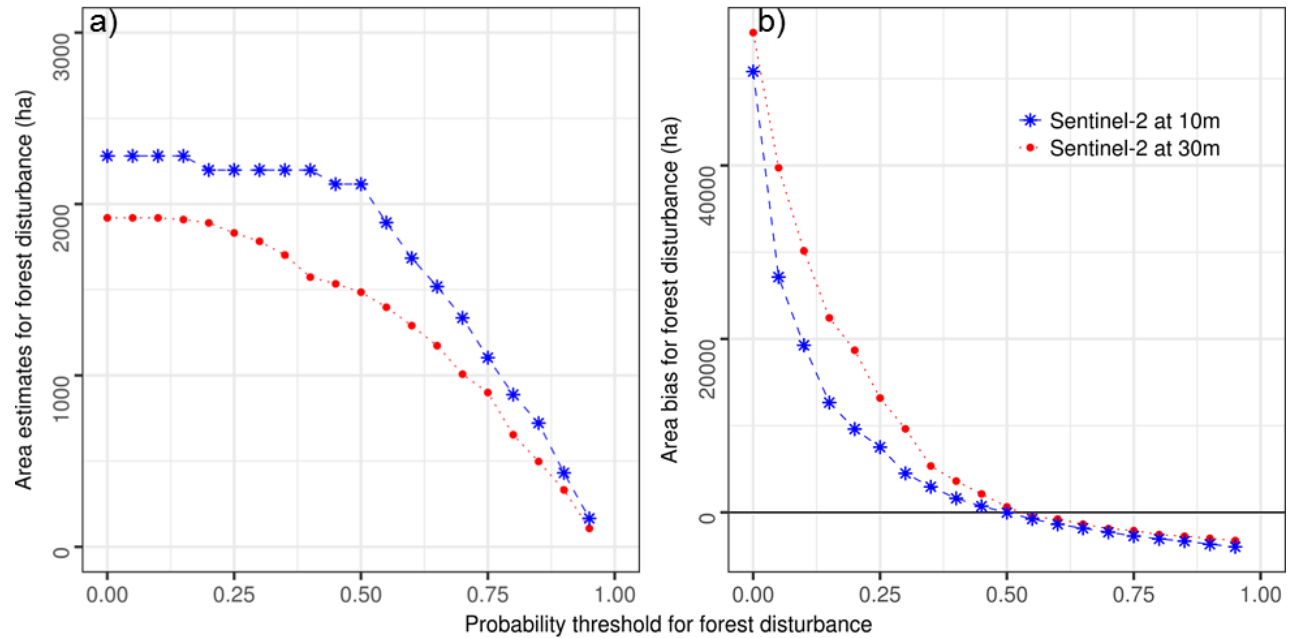

Figure 5.8: Area estimates (a) and area bias (b) for forest disturbances detected from Sentinel-2 NDVI (normalised difference vegetation index) time series at $10 \mathrm{~m}$ and $30 \mathrm{~m}$ resolutions in UNESCO Kafa Biosphere Reserve, southwestern Ethiopia while using Landsat and Sentinel-2 NDVI time series for 2010 -2015 as historical reference. 


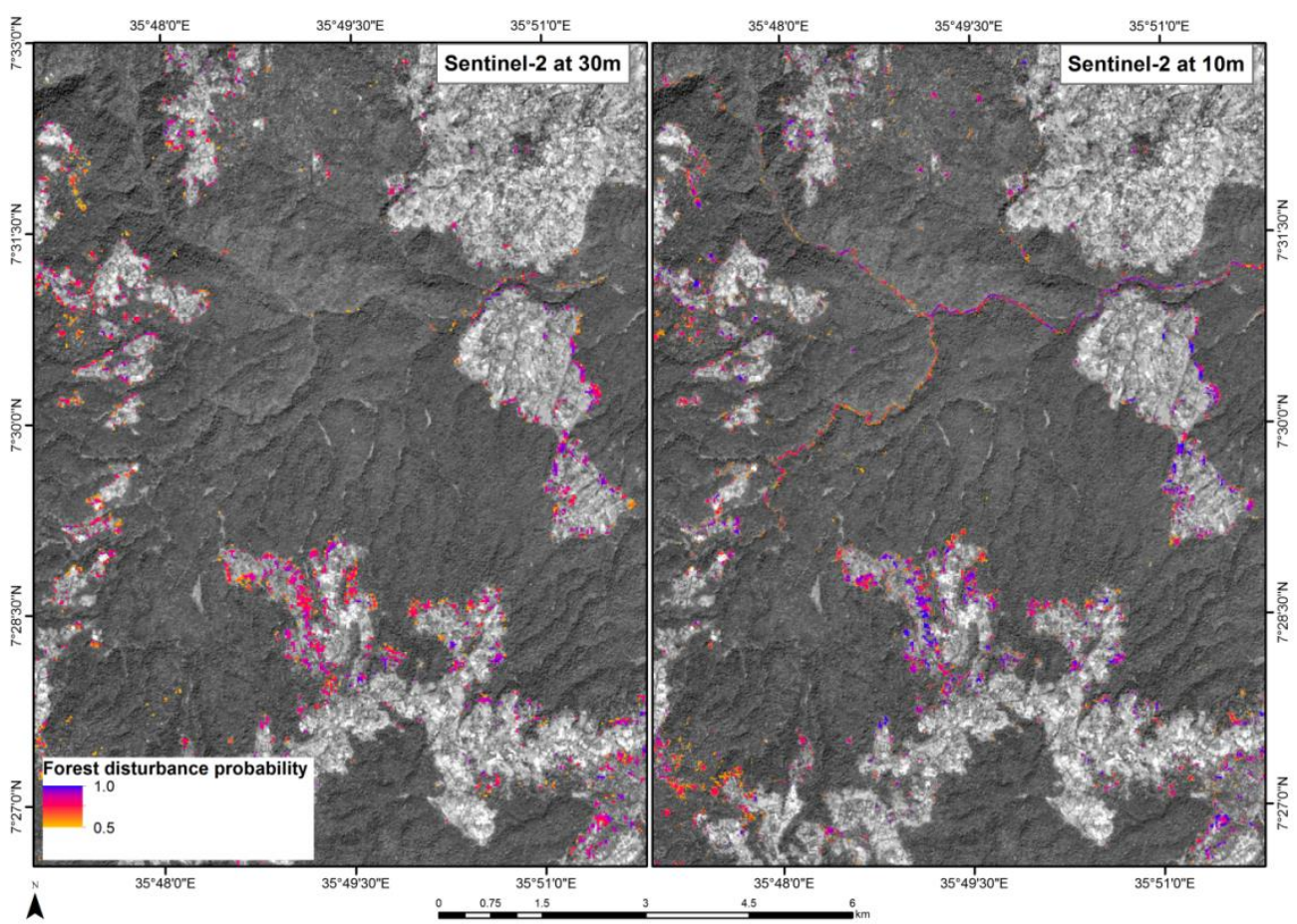

Figure 5.9: An example for forest disturbances detected from Sentinel-2 NDVI time series at $10 \mathrm{~m}$ and $30 \mathrm{~m}$ resolutions in Kafa biosphere reserve while using Landsat and Sentinel-2 NDVI time series for 2010 -2015 as historical reference. Note the river channels which were omitted by the $30 \mathrm{~m}$ resolution scenario but clearly detected by $10 \mathrm{~m}$ resolution scenario. These river channels have been there prior to year 2016, but given its narrow nature, it does not appear as a non-forest area in a $30 \mathrm{~m}$ resolution image. This example illustrates how spatial resolution affects the detection of forest disturbances from satellite image time series.

\subsubsection{Effect of observation density on detection of forest disturbances}

Combining Landsat and Sentinel-2 time series increased the number of valid observations available per pixel in the monitoring period (Figure 5.10). Increasing the observation density of the improved the detection of forest disturbances (Figure 5.11). For example, at the probability threshold of 0 , the producer's accuracy for Landsat-only was only $55.6 \pm 8.4 \%$ (area estimates $=$ $1862.6 \pm 155.7 \mathrm{ha}$ ). This producer's accuracy increased to $81 \pm 11.9 \%$ (area estimates $=2741.1 \pm 327.3 \mathrm{ha})$ when using a combined time series of Landsat and Sentinel-2. The producer's accuracy for forest disturbances, however, decreased with increasing probability threshold, reaching $25.4 \pm 2.6 \%$ (area 
estimate $=851.2 \pm 21.8 \mathrm{ha}$ ) for Landsat-only scenario and $8.2 \pm 0.5 \%$ (area estimates $=273.3 \pm 5.8$ ha) for Landsat + Sentinel-2 scenario at probability threshold of 0.95. At lower probability thresholds, the area bias (in favour of the commission error) was higher when using a combined time series of Landsat and Sentinel-2 than when using Landsat-only (Figure 5.12). This area bias, however, decreased exponentially when increasing the probability threshold from 0 to 0.95. For Landsat-only scenario, the lowest area bias (-124.2ha) was achieved at probability threshold of 0.65 , whereas the lowest area bias (-39.5 ha) for Landsat + Sentinel-2 scenario was achieved at probability threshold of 0.55 . At the lowest area bias, the producer's accuracy for Landsat-only scenario was $51.8 \pm 3.0 \%$ (area estimate $=1735.7 \pm 52.7 \mathrm{ha})$, and $67.0 \pm 3.1 \%$ (area estimate $=2245.1 \pm 68.6$ ha) for Landsat + Sentinel-2 scenario. Figure 5.13 shows an example of forest disturbances detected when using Landsat only, and additional forest disturbances detected after combining Landsat and Sentinel-2 time series.

The impact of combining Landsat and Sentinel-2 time series on temporal accuracy was marginal. At the crossover points (lowest area bias), the temporal detection delay for Landsat only scenario was 21 days, and only decreasing to 17 days after combining Landsat and Sentinel-2 time series.

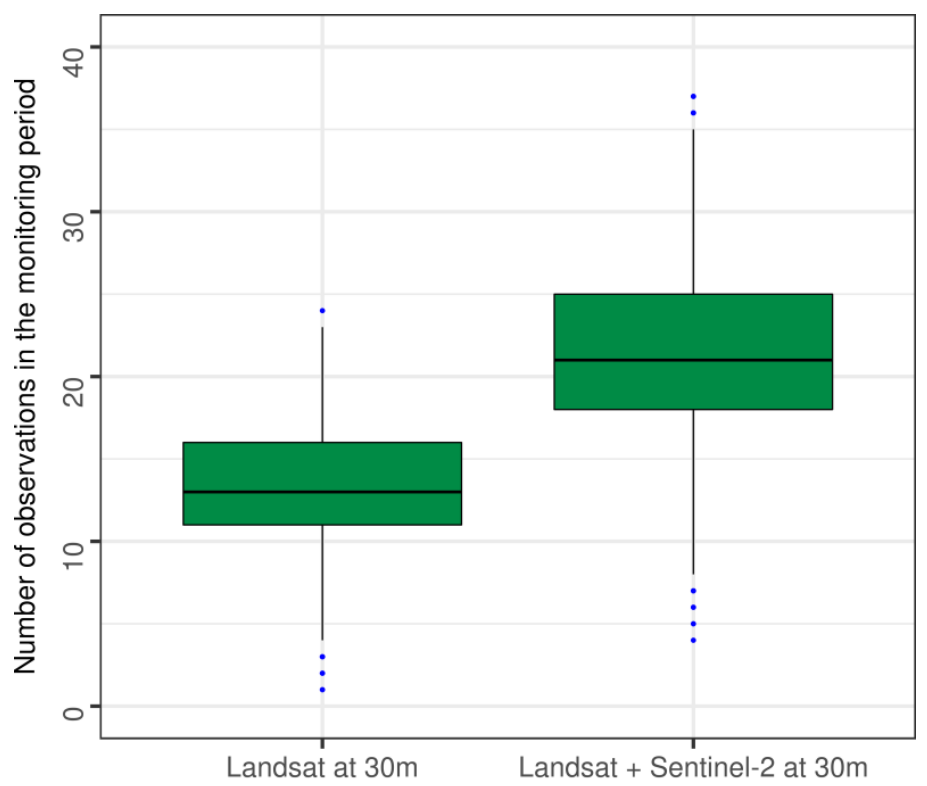

Figure 5.10: The number of valid observations in the monitoring period (01- 012016 to 31-12-2016) over UNESCO Kafa Biosphere Reserve, southwest of Ethiopia for Landsat-only and Landsat + Sentinel-2 scenarios. The number of valid observations is based on 2000 validation sample pixels. 


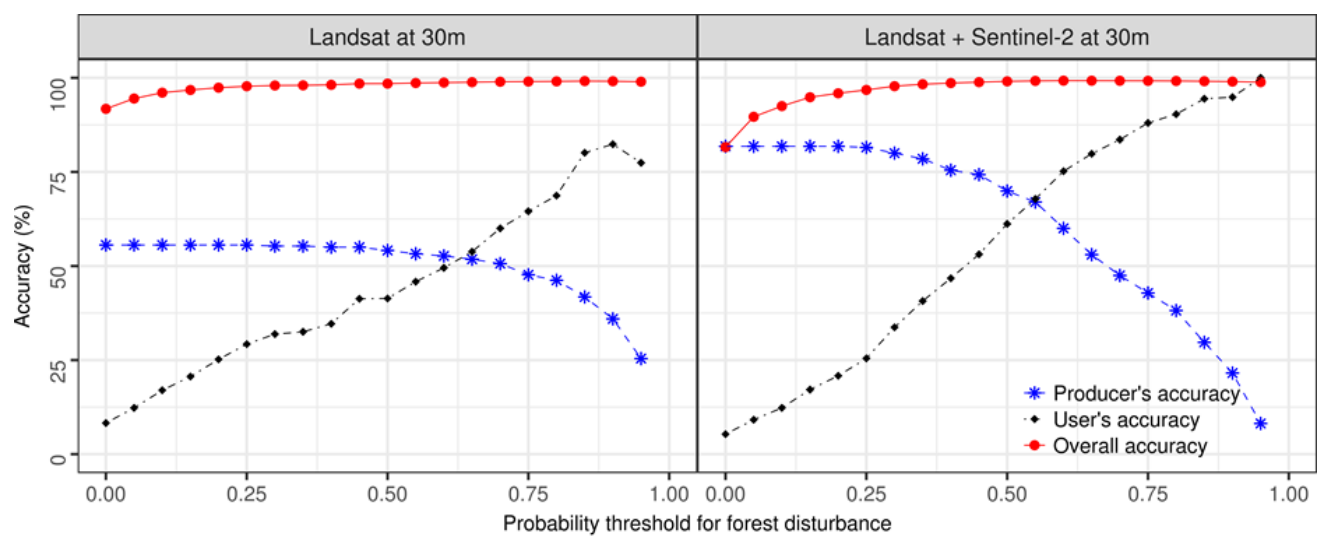

Figure 5.11: Overall accuracy, user's accuracy and producer's for forest disturbances detected in UNESCO Kafa Biosphere Reserve, southwestern Ethiopia in 2016 from normalised difference vegetation index (NDVI) time series derived from Landsat and Sentinel-2 data. Accuracies are for Landsat-only (Landsat-7/ETM+ and Landsat-8/OLI) and Landsat +Sentinel-2 scenarios.

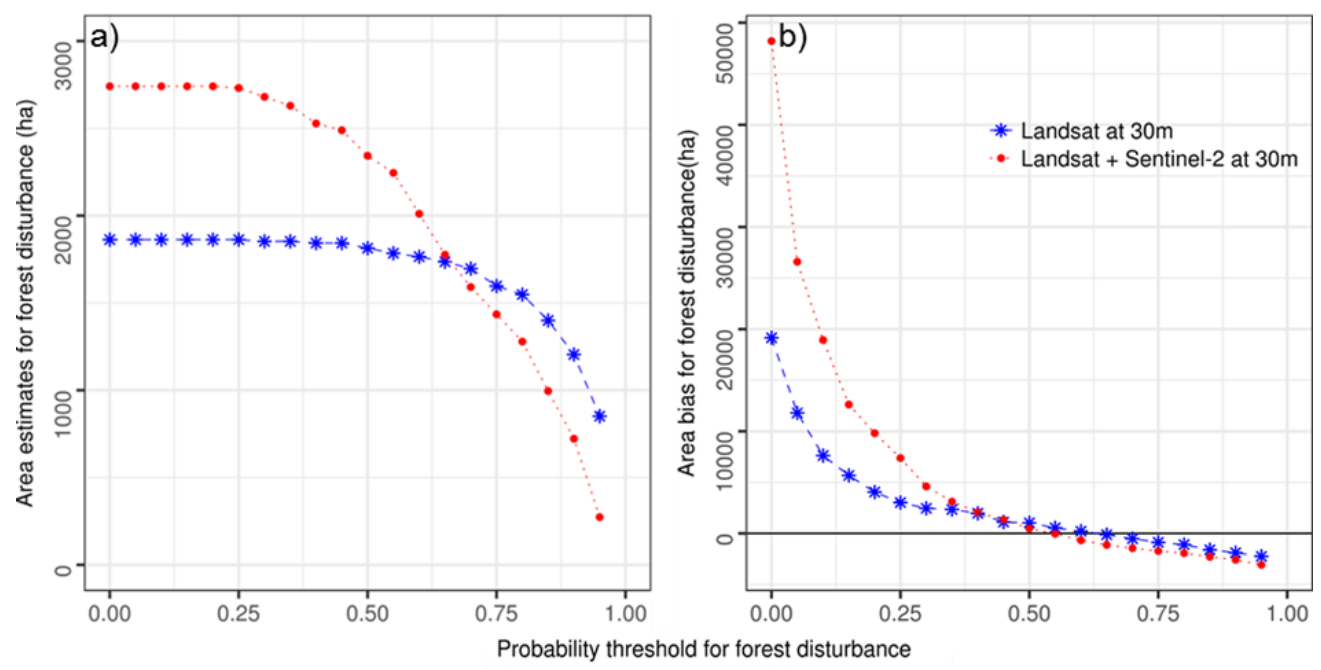

Figure 5.12: Area estimates (a) and area bias (b) for forest disturbances detected in UNESCO Kafa Biosphere Reserve, southwestern Ethiopia in 2016 from normalised difference vegetation index (NDVI) time series derived from Landsat and Sentinel-2 data. Area estimates are for Landsat-only (from Landsat-7/ETM+ and Landsat-8/OLI) and Landsat +Sentinel-2 scenario. 


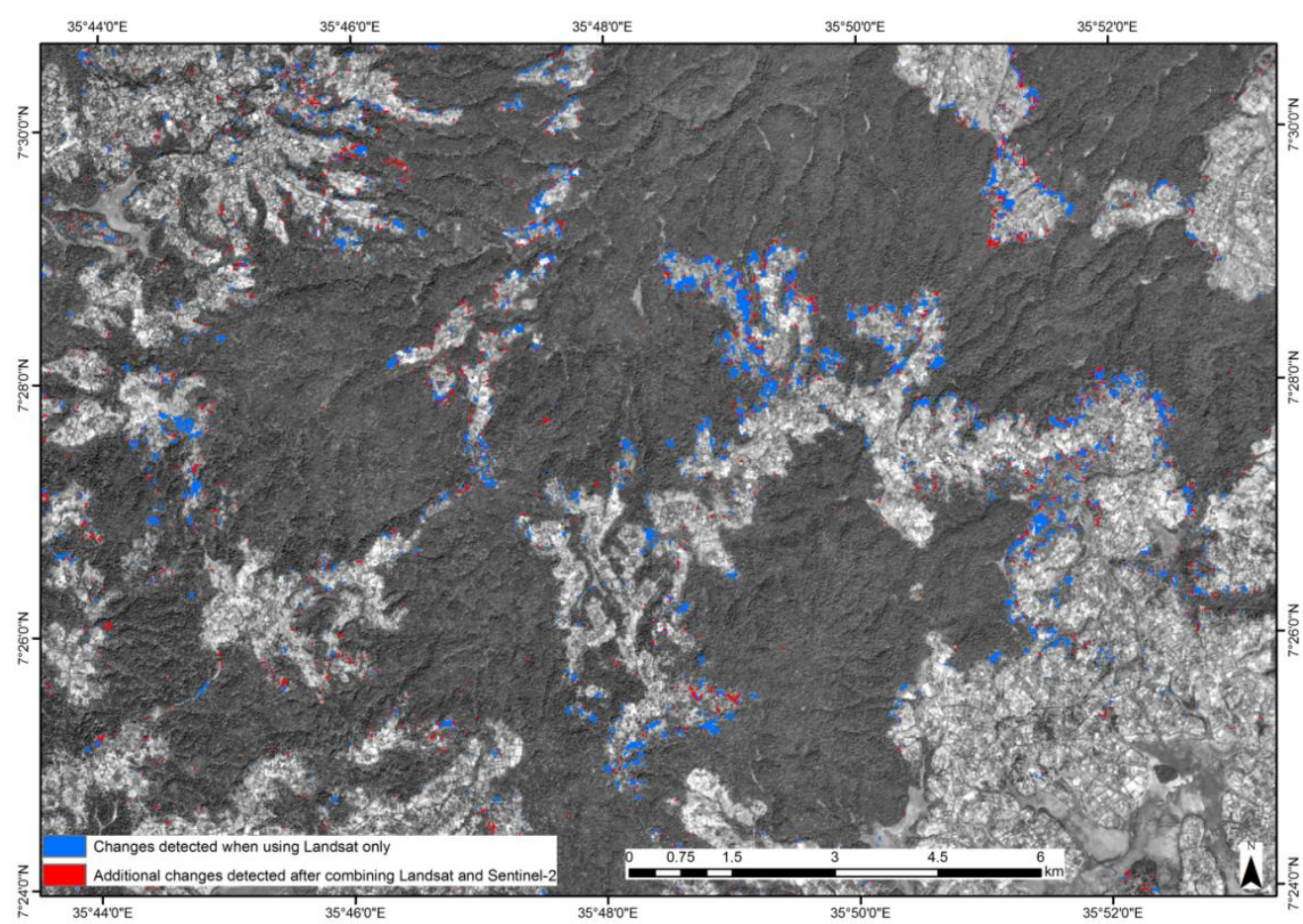

Figure 5.13: An example illustrating the complementarity of Landsat and Sentinel-2 for detection of forest disturbances. Additional forest disturbances (in red) were detected after combining Sentinel-2/MSI time series. Forest disturbances (in blue) were detected when using Landsat time series only (Landsat-7/ETM+ and Landsat-8/OLI). Forest disturbances were detected in UNESCO Kafa Biosphere Reserve, southwestern Ethiopia between 01- 01-2016 and $31-12-2016$ at $30 \mathrm{~m}$ resolution using normalised difference vegetation index (NDVI).

\subsubsection{Comparison of overall accuracy and disturbance area estimates for all four monitoring scenarios at crossover point}

The overall accuracy and area estimates of forest disturbance achieved from each monitoring scenario (Landsat at 30m, Sentinel-2 at 30m, and Landsat + Sentinel-2 at 30m, Sentinel-2 at 10m) at crossover point (lowest area bias) are shown in Figure 5.14. The difference in overall accuracy between all scenarios was not significant, but area estimates were significantly different. Detecting forest disturbances at $30 \mathrm{~m}$ resolution in Sentinel-2 time series only (Sentinel-2 at $30 \mathrm{~m})$ achieved the lowest area estimates $(1397.9 \pm 40.5 \mathrm{ha})$ at the crossover point, followed by Landsat-only scenario (Landsat at $30 \mathrm{~m}$ ) which achieved area estimates of $1735.7 \pm 52.7$ ha. The highest area estimates $(2245.1 \pm 68.6$ ha $)$ at 
the crossover point was achieved from Landsat + Sentinel-2 at $30 \mathrm{~m}$, but this estimate was not significantly different from that achieved from Sentinel-2 at $10 m$ scenario $(2115.9 \pm 65.4 \mathrm{ha})$.

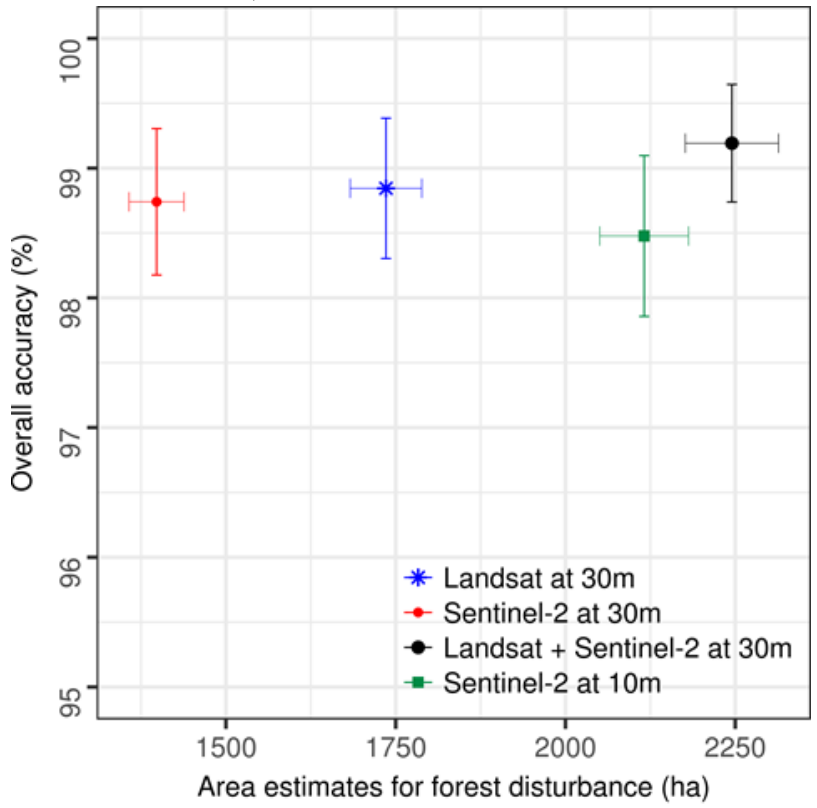

Figure 5.14: Overall accuracy and area estimates for forest disturbance achieved from each monitoring scenario at the crossover point (lowest area bias) when detecting forest disturbances in UNESCO Kafa Biosphere Reserve, southwestern Ethiopia in 2016 from normalised difference vegetation index (NDVI) time series derived from Landsat and Sentinel-2 data.

\subsection{Discussion}

In this chapter, we combined Landsat and Sentinel-2 time series to evaluate how spatial resolution and observation density affects detection of small-scale forest disturbances. We also evaluated whether spatial normalisation can reduce the inter-sensor differences in Landsat-Sentinel-2 NDVI time series significantly to create a temporally consistent multi-sensor NDVI time series suitable for forest change detection. Our results show that the inter-sensor differences in Landsat-Sentinel-2 NDVI time series can be reduced significantly through spatial normalisation. Spatial normalisation did not completely remove the differences, but it reduced them significantly, resulting into temporally consistent time series suitable for forest change detection. Previous studies demonstrated that spatial normalisation reduces seasonality in image time series (Hamunyela, Verbesselt, \& Herold, 2016; Reiche et al., in review), but it was not known whether it can also reduce inter-sensor differences in multi- 
sensor time series. Our findings, therefore, indicate that spatial normalisation is an important pre-processing step in satellite-based forest change monitoring because it can reduce both seasonality and inter-sensor differences in satellite image time series. Reducing inter-sensor differences and seasonality in multisensor time series through spatial normalisation is more attractive for forest change monitoring because it allows for rapid combination of data from multiple sensors for forest change detection in different forest types. Unlike the adjustment factors which are commonly used to harmonise observations from multiple satellites (Roy et al. 2016; Fan \& Liu 2017), spatial normalisation simplifies the process of combining data from multiple satellites for forest change detection, because it eliminates the need for using the time series of one sensor as a reference for other sensors. However, spatial normalisation may face challenges in areas where evergreen and deciduous forests co-exist because $\mathrm{P}_{95}$ is likely to be calculated from pixels from evergreen forest (Hamunyela, Verbesselt, Bruin, et al. 2016). This problem of co-existence of evergreen and deciduous forest can be solved by normalising each pixel based on forest type.

Our analysis show that mapping forest disturbances at $10 \mathrm{~m}$ resolution leads to more accurate forest change detection than mapping forest disturbances at $30 \mathrm{~m}$ resolution (Figure 5.9). We observed that small-scale disturbances (e.g. road networks), as well as river channels within the forest, which were not detectable from $30 \mathrm{~m}$ resolution, were detected with high confidence at $10 \mathrm{~m}$ resolution (Figure 5.9). The ability to detect small-scale disturbances such as road networks may explain why the area estimates for forest disturbance estimated from Sentinl-2 at $10 \mathrm{~m}$ resolution were much higher than those estimated from Sentinel-2 at $30 \mathrm{~m}$ resolution. These findings, therefore, highlight the importance of high spatial resolution data from Sentinel-2 sensors within the context of forest change monitoring. In particular, we demonstrated that free and open access $10 \mathrm{~m}$ resolution data from Sentinel-2 has high potential to improve our capacity to map forest disturbances occurring at small spatial scale. In the Landsat-only era, detecting small-scale forest disturbances has been challenging, limiting our capacity to quantify global forest loss accurately (Tyukavina et al. 2013). However, with the $10 \mathrm{~m}$ Sentinel-2 data available globally, there is now a unique opportunity to improve global estimates of forest loss. In our monitoring context, detection of river channels is a commission error, but detecting these channels robustly demonstrates the importance of using high spatial resolution satellite time series in land monitoring. It also highlights the potential for Sentinel-2 sensors to improve our understanding of different dynamics occurring on land surface.

With two Sentinel-2 sensors now available, the $10 \mathrm{~m}$ resolution time series from Sentinel-2 also has potential to enhance the capacity to detect forest disturbances which are caused, for example, by logging roads and selective logging early. Although Sentinl-2 time series currently lacks sufficient historical observations, we showed in this chapter that Landsat time series disaggregated 
to $10 \mathrm{~m}$ resolution can be used as a reliable historical reference for Sentinel-2 time series. Therefore, there is no need to wait for several years before exploiting Sentinel-2 time series to map forest disturbances at sub-annual scales. Detecting forest disturbances at $10 \mathrm{~m}$ resolution is, however, computationally demanding, hence posing a huge challenge for large-area forest monitoring. Computationally powerful platforms, e.g. Google Earth Engine (https://earthengine.google.com/), may therefore play a crucial role in achieving sub-annual forest change mapping and monitoring over large area using $10 \mathrm{~m}$ resolution Sentinel-2 time series.

Combining all available Landsat and Sentinel-2 observations at $30 \mathrm{~m}$ resolution resulted in more accurate forest change detection than when using Landsat only (Landsat-7/ETM+ and Landsat-8/OLI). More specifically, our results show that combining observations from multiple sensors to increase observation density in image time series does not only improve temporal accuracy, but it also improves the area estimates for forest disturbance. These findings corroborate previous studies (Reiche, de Bruin, et al. 2015; Reiche et al. 2016; Reiche, Verbesselt, et al. 2015) which showed that combining observations from multiple satellite sensors increases the accuracy of forest change detection. However, combining time series from multiple sensors can increase the noise in the time series, resulting to high commission error. In our case, the high commission error when combining Landsat and Sentinel-2 NDVI time series could be attributed to imperfect cloud and shadow masking for Sentinel-2 data. Nonetheless, we observed that the commission error also increased when combining observations from Landsat-7/ETM+ and Landsat-8/OLI only. To avoid this high commission error, change detection approaches should have capacity to reject false detections when detecting forest disturbances from multi-sensor time series. We observed that the commission error decreased exponentially when increasing the probability threshold for forest disturbance. This exponential decrease in the commission error corroborates our previous findings (Hamunyela et al., in review) that using a set of space-time features to confirm forest disturbances increases the capacity to reject false detections.

For all monitoring scenarios we implemented in this chapter Sentinel-2 at $30 \mathrm{~m}$ scenario produced the lowest disturbance area estimates when aiming for a disturbance map with the lowest area bias, followed by Landsat at 30m scenario. Area estimates from Sentinel-2 at 30m scenario were lower than those estimated from Landsat at $30 \mathrm{~m}$ because Sentinel-2 at $30 \mathrm{~m}$ scenario had lower observation density than Landsat at $30 \mathrm{~m}$ scenario. For Sentinel-2 at 30m, forest disturbances were detected using observations from a single sensor (Sentinel2/MSI) whereas the scenario for Landsat at 30m combined observations from two sensors (Landsat-7/ETM+ and Landsat-8/OLI). The area estimates for forest disturbance increased further after combining all available observations from Landsat-7/ETM+, Landsat-8/OLI and Sentinel-2/MSI at 30m resolution, thus confirming that increasing observation density in the image time series increases the accuracy for forest change detection (Reiche, de Bruin, et al. 2015; 
Reiche, Verbesselt, et al. 2015). Disturbance area estimates from Sentinel-2 at $10 \mathrm{~m}$ resolution were higher than those from Sentinel-2 at 30m and Landsat at $30 \mathrm{~m}$ scenarios, despite it having similar observation density as Sentinel-2 at $30 \mathrm{~m}$, and lower density than that of Landsat at $30 \mathrm{~m}$. These findings show that the use of high spatial resolution data improves the detection of small-scale forest disturbances significantly. The disturbance area estimates from Sentinel-2 at $10 \mathrm{~m}$ scenario were nonetheless not significantly different from those estimated from a combined time series of all available Landsat-7/ETM+, Landsat-8/OLI and Sentinel-2/MSI observations at 30m resolution. For Sentinel2 at $10 \mathrm{~m}$ scenario, some forest disturbances, which were detected in Landsat + Sentinel-2 at 30m scenario, might have been omitted because there were not two consecutive anomalies in time series due to limited observation density. These findings, therefore, show that mapping forest disturbances using high spatial resolution data does not supersede the importance of dense time series when monitoring forest disturbances from satellite data. Dense time series is critically important, especially for change detection approaches which only confirm forest disturbance when there are at least two consecutive negative anomalies in the time series. With a Sentinel-2B/MSI launched successfully, free and open access dense time series at $10 \mathrm{~m}$ resolution will become available. Such high resolution (spatially and temporally) data will enhance our capacity to map small-scale forest disturbances accurately and timely.

\subsection{Conclusion}

Mapping small-scale forest disturbances accurately and timely remains one of the major challenges for satellite-based forest monitoring. This chapter demonstrated that mapping of small-scales can be improved by using the $10 \mathrm{~m}$ resolution time series from recently launched Sentinel-2 sensors. Monitoring forest disturbances at $10 \mathrm{~m}$ resolution improved the detection of small-scale disturbances significantly when compared to $30 \mathrm{~m}$ resolution. Combining all available Landsat-7/ETM+, Landsat-8/OLI and Sentinel-2/MSI observations at $30 \mathrm{~m}$ resolution also improves the detection of forest disturbances when compared to Landsat-only scenario, thus emphasis the complementarity between Landsat and Sentinel-2 sensors. Disturbances area estimates from $10 \mathrm{~m}$ resolution Sentinel-2 were not significantly different from those estimated from a combined time series of all available Landsat-7/ETM+, Landsat-8/OLI and Sentinel-2/MSI observations at $30 \mathrm{~m}$ resolution, thus highlighting the importance of using high spatial resolution and dense time series when monitoring small scale forest disturbances. Spatial normalisation is not only suitable approach for reducing seasonality in image time series, but it is also a promising approach for reducing inter-sensor differences in multi-sensor time series when combining observations from multiple optical satellite sensors. With two Sentinel-2 sensors now available, the observation density for the $10 \mathrm{~m}$ resolution time series will 
increase globally, providing an opportunity to improve the estimates of global forest loss. However, processing and analysing the $10 \mathrm{~m}$ resolution time series is computationally demanding when compared to the 30 resolution. Therefore computationally powerful cloud computing platforms (e.g. Google Earth Engine) will crucial in facilitating large-area and frequent monitoring of forest disturbances using the $10 \mathrm{~m}$ resolution time series from Sentinel-2 sensors.

\section{Acknowledgements}

This work was funded by the European Space Agency (ESA) ForMoSa project Forest Degradation Monitoring with Satellite Data Project (grant agreement 5160957022), the European Commission Horizon 2020 BACI project (grant agreement 640176), European Commission Horizon 2020 LANDSENSE project (grant agreement number 689812) and JRC CGLOPS1 (grant agreement FWC199494). 


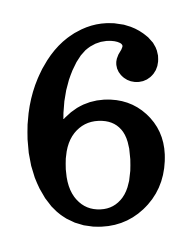

Improving near real-time deforestation monitoring in tropical dry forests by combining dense Sentinel-1 time series with Landsat and ALOS-2 PALSAR-2

This chapter is based on:

Reiche, J., Hamunyela, E., Verbesselt, J., Hoekman, D., \& Herold, M. Improving near-real time deforestation monitoring in tropical dry forests by combining dense Sentinel-1 time series with Landsat and ALOS-2 PALSAR-2. Remote Sensing of Environment, in review. 


\section{Abstract}

Combining observations from multiple optical and synthetic aperture radar (SAR) satellites can provide temporally dense and regular information at medium resolution scale independently of weather, season and location. This has the potential to improve near real-time deforestation monitoring in dry tropical regions, where traditional optical only monitoring systems typically suffer from limited data availability due to persistent cloud cover. In this context, the recently launched Sentinel-1 satellites promise unprecedented potential as for the first time dense and regular SAR observation are available free and openly. Here, we demonstrated multi-sensor near real-time deforestation detection in tropical dry forests, for which we combined Sentinel1 C-band SAR time series with ALSO-2 PALSAR-2 L-band SAR and Landsat 7 and 8 . We used spatial normalisation to reduce the dry forest seasonality in the SAR and optical time series, and combined them within a probabilistic approach to detect deforestation in near real-time. Our results for a dry tropical forest site in Bolivia $\left(10,000 \mathrm{~km}^{2}\right)$ showed that, as a result of high observation availability of Sentinel-1, deforestation events were detected more timely with Sentinel-1 than compared to Landsat and PALSAR-2. The spatial and temporal accuracies further improved beyond the single-sensor results when combining observations in a multi-sensor approach. We improved the precision of the reference data derived from the multi-sensor satellite time series, which enabled a more robust estimation of the temporal accuracy. We quantified how the near real-time deforestation detection is associated with a trade-off between the confidence in detection and the temporal accuracy. We showed that the trade-off affects the choice on how to use the near-real time data for different applications such as fast alerting with high temporal accuracy but lower confidence versus accurate detection at lower temporal detail. When aiming for a high confidence in change area estimates for example, deforestation was detected with a user's accuracy of $88 \%$ and producer's accuracy of $89 \%$ (low area bias) and a mean time lag of 31 days using all sensors. This is on average 7 days earlier than when using only Sentinel-1 observations and six weeks earlier than when relying on Landsat observations only. We showed that confident near real-time deforestation alerts can be provided with a mean time lag of 22 days but these are associated with a higher commission error. With more dense time series data expected from the Sentinel-1 and -2 sensors for the upcoming decade, spatial and temporal detection accuracy of multi-sensor deforestation monitoring in the tropics will improve further.

Keywords: Sentinel-1, Multi-sensor, Near real-time, ALOS-2 PALSAR-2, Landsat, Temporal accuracy, Time series, Change detection, Deforestation, Sensor interoperability, SAR-optical, Dry forest 


\subsection{Introduction}

Satellite-based monitoring systems are the primary tools for providing near real-time (NRT) information on newly deforested areas in vast and inaccessible tropical forests. Their potential to empower governments and communities to enact timely actions against illegal and unsustainable forest activities and to respond to natural disasters is increasingly recognized (Assunção et al. 2013; Lynch et al. 2013; Wheeler et al. 2014; Hansen et al. 2016). At an operationalised level, the NRT detection of deforestation is currently realized by monitoring systems that mainly rely on near daily observations of the coarse resolution MODIS (Moderate Resolution Imaging Spectroradiometer) sensor. The near daily observations provide a satisfactory temporal coverage in the frequently cloud covered tropical region (Diniz et al. 2015; Shimabukuro et al. 2006; Hammer et al. 2009; Wheeler et al. 2014). Due to the coarse resolution of MODIS data of $250-500 \mathrm{~m}$, however, many small scale changes are missed (Anderson et al. 2005; Hansen \& Loveland 2012; Hammer et al. 2014). Hansen \& Loveland (2012) demonstrated that MODIS misses up to fifty percent of the forest changes when compared to medium resolution Landsat data $(30 \mathrm{~m})$.

Stimulated by the opening of the Landsat archive in combination with the ability to download fully pre-processed images, efforts in recent years shifted towards operational and large area Landsat based deforestation monitoring at annual (Hansen et al. 2013; Souza, Jr et al. 2013) and sub-annual scale (Hansen et al. 2016). Several optical time series approaches with NRT capabilities have been developed to exploit the entire temporal detail of Landsat data (Verbesselt et al. 2012; Xin et al. 2013; Zhu et al. 2012; Hansen et al. 2016). Hansen et al. (2016) demonstrated the potential for and constraints of operational Landsat based deforestation alerts for the humid tropics. The major limitation of Landsat-based tropical deforestation alerts is the limited availability of cloud-free observations (Sannier et al. 2014; Hansen et al. 2016; Souza, Jr et al. 2013). In particular cloud-free observations are rare during the wet season. Other regions, such as the Peruvian cloud forests for example (Hansen et al. 2016), suffer from pervasive cloud cover throughout the entire year. In extreme cases, Landsat data gaps even remain for more than one year (Potapov et al. 2012; Hansen et al. 2016; Sannier et al. 2014). In summary, a monitoring system that relies on medium resolution optical data only will not provide a sufficient number of cloud-free observations throughout all seasons and geographical locations. A reduced number of cloud-free observations results in delayed detection of new deforestation events (Reiche, Verbesselt, et al. 2015).

Synthetic aperture radar (SAR) can penetrate through clouds, and therefore has the potential to complement optical-based forest monitoring systems (Joshi et al. 2016; Vaglio Laurin et al. 2013; De Sy et al. 2012). To monitor tropical deforestation at larger scales mainly long wavelength L-band 
SAR $(\sim 23.5 \mathrm{~cm})$ data has been utilized (Shimada et al. 2014; Whittle et al. 2012). Shorter wavelength C-band $(\sim 5.6 \mathrm{~cm})$ SAR are generally less useful for forest change monitoring because of the lower penetration depth and rapid saturation of the signal over forests (Woodhouse 2005). Fragmented and inconsistent data acquisitions and/or commercial data distribution of key SAR missions in the past hampered their operational application and limited opportunities to integrate SAR and optical data (Reiche et al. 2016). With the launch of the Sentinel-1A and 1B C-band SAR satellites in 2014 and 2016 (Torres et al. 2012), for the first time, dense SAR time series are free and openly available for the tropical region. Since temporal sampling frequency is key to NRT deforestation monitoring, Sentinel-1 could be a milestone when it comes to more precise tracking of forest change events and activities - a potential that is yet to be explored. A key question is to what extent the high temporal observation density of Sentinel-1 C-band SAR may compensate for the lower sensitivity to detect deforestation when compared to longerwavelength L-band SAR observations. L-band SAR data are currently available from the ALOS-2 PALSAR-2 mission (launched 2014, Rosenqvist et al. 2014), but only a few images per year are available for most tropical regions and the commercial data distribution limits the operational uptake. However, freely accessible L-band data are in sight with the upcoming SAOCOM-1 (Satélite Argentino de Observación Con Microondas; planned launch 2017) and NISAR (NASA/ISRO Synthetic Aperture Radar; planned launch 2020) missions.

Space agencies and international organisations, i.e. the Global Forest Observation Initiative (GFOI 2015), emphasize the need and potential for joint exploitation of the impeding stream of free-of-charge C-band SAR, L-band SAR and optical time series to improve tropical forest monitoring (Reiche et al. 2016). Using dense Sentinel-1 time series for NRT deforestation monitoring could provide a significant step forward in its own right. Combining time series from multiple SAR and optical sensors, however, has the potential to even improve the robustness of NRT deforestation monitoring at medium resolution scale by increasing the number of available observations and ensuring a minimum number of observations for all seasons and geographical locations. In recent years several studies have demonstrated the increase of spatial and temporal accuracy of deforestation detection when combining SAR and optical time series (Lehmann et al. 2012; Lehmann et al. 2015; Reiche et al. 2013; Reiche, de Bruin, et al. 2015; Reiche, Verbesselt, et al. 2015). These studies have developed some of the methodological underpinnings for the combination of these datasets using probabilistic approaches (Lehmann et al. 2012; Lehmann et al. 2015; Reiche, de Bruin, et al. 2015), and focused on combining Landsat and ALOS PALSAR L-band SAR data at small test sites and humid tropical forest conditions. The expansion of these methods, however, to seasonal dry tropical forest conditions, to combine more than two sensors, and the consideration of new dense Sentinel-1 C-band SAR data, is outstanding. 
Detecting deforestation in satellite image time series in dry tropical forest conditions requires the removal of the seasonal forest component. Otherwise, the seasonal variations may lead to substantial false detection of deforestation (Hamunyela, Verbesselt \& Herold 2016). While the seasonal forest component in the optical time series signal is driven by changes in the photosynthetic phenology (Tucker 1979), changes in canopy structure and moisture cause seasonal variations in the SAR backscatter signal (Ulaby et al. 1986). The majority of time series approaches proposes seasonal model fitting to account for forest seasonality (Verbesselt, Hyndman, Newnham, et al. 2010; Zhu et al. 2012). Robust model fitting requires sufficient historical observations. Due to cloud cover, historical Landsat time series in tropical regions are often too sparse for robust fitting of seasonal model. The same applies for ALOS PALSAR-1/2 L-band SAR time series. Hamunyela, Verbesselt \& Herold (2016) proposed spatial normalisation to reduce seasonality in Landsat time series and successfully applied it to a dry tropical forest. Each pixel is normalised with the value of dense forest in the spatial surrounding of the pixel to be normalised. The application of spatial normalisation could also be applied in SAR time series to reduce seasonal variability; a potential that has yet to be demonstrated.

Assessing the temporal accuracy alongside the spatial accuracy gets increasingly important when evaluating NRT monitoring systems. While consolidated guidelines and methods exist for estimating the spatial accuracy of detected change (Foody 2002; Olofsson et al. 2013; Stehman 2009; Olofsson et al. 2014), assessing the temporal accuracy is challenging due to the lack of temporally dense reference information. As a result, reference data are commonly derived from the satellite time series itself using visual image interpretation (Cohen et al. 2010; Zhu et al. 2012; Devries, Verbesselt, et al. 2015). Commonly, the date at which the change is first visible in the image time series is used as reference data to calculate the temporal accuracy or temporal detection delay (Zhu \& Woodcock 2014a; Pratihast et al. 2015; Hansen et al. 2016; Reiche, Verbesselt, et al. 2015; DeVries et al. 2015). This approach results in time-biased reference data. The bias is related to the fact that true date of deforestation can occur at any date between the date of the image at which the change is first visible (commonly considered as reference date) and the date of the previous image in the time series. This imprecision is variable in time and space, and becomes even larger when relying on sparse and/or irregular time series, such as for example Landsat in some tropical regions. Since NRT monitoring is aiming for temporal accuracies in order of weeks or days, such an imprecision in the reference data becomes critical when assessing the performance for various applications. Despite the fundamental importance, the consideration of the temporal precision in the reference data, and the impact for users, have not been thoroughly studied for forest change alerting. 
In this chapter we address some key challenges for multi-sensor NRT deforestation monitoring building on newly available dense Sentinel-1 time series data in combination with Landsat and PALSAR-2. We aim to:

(i) Demonstrate how spatial normalisation reduces dry forest seasonality in Sentinel-1, ALOS-2 PALSAR-2 and Landsat time series in order to combine them for NRT deforestation detection using a probabilistic approach. We put particular emphasis on how environmental effects and dry forest seasonality affect the dense Sentinel-1 C-band SAR time series signal when compared to Landsat NDVI and PALSAR-2 L-band SAR.

(ii) Compare the spatial and temporal accuracy of single-sensor Sentinel-1, PALSAR-2 and Landsat results versus multi-sensor results. We take the precision of the reference data into account to provide a more robust estimate on how quickly a change event happening on the ground can be detected.

(iii) Evaluate the impact of uncertainties for different user scenarios by taking a critical look at the trade-off between spatial and temporal accuracy associated with NRT deforestation monitoring.

With this study we highlight the unprecedented potential of dense Sentinel-1 time series and its combination with other SAR and optical sensors to improve the robustness of NRT deforestation monitoring in dry tropical forest environment.

\subsection{Study area}

The study was conducted at a dry tropical forest site $\left(10,000 \mathrm{~km}^{2}\right)$, located in the southeast of the province of Santa Cruz, Bolivia (centred at $18.388^{\circ} \mathrm{S}$, $62.361^{\circ} \mathrm{W}$ ) (Figure 6.1). Being one of the wettest regions of Bolivia, this area is characterized by a humid tropical climate with distinct wet ( October - May) and dry seasons ( June - September). The change from wet and dry seasons is associated with a strong change in photosynthetic activity of the forest. Deforestation in the area is mainly caused by large-scale industrial logging and agricultural expansion, resulting into visible patches of land cleared from forests (Figure 6.1). While deforestation is defined in many ways, we follow a forest cover definition in a sense that pixels with less than $10 \%$ canopy cover are considered non-forest. Since this is a study for near-real time detection, a focus on the pixel level is our preferred choice. 


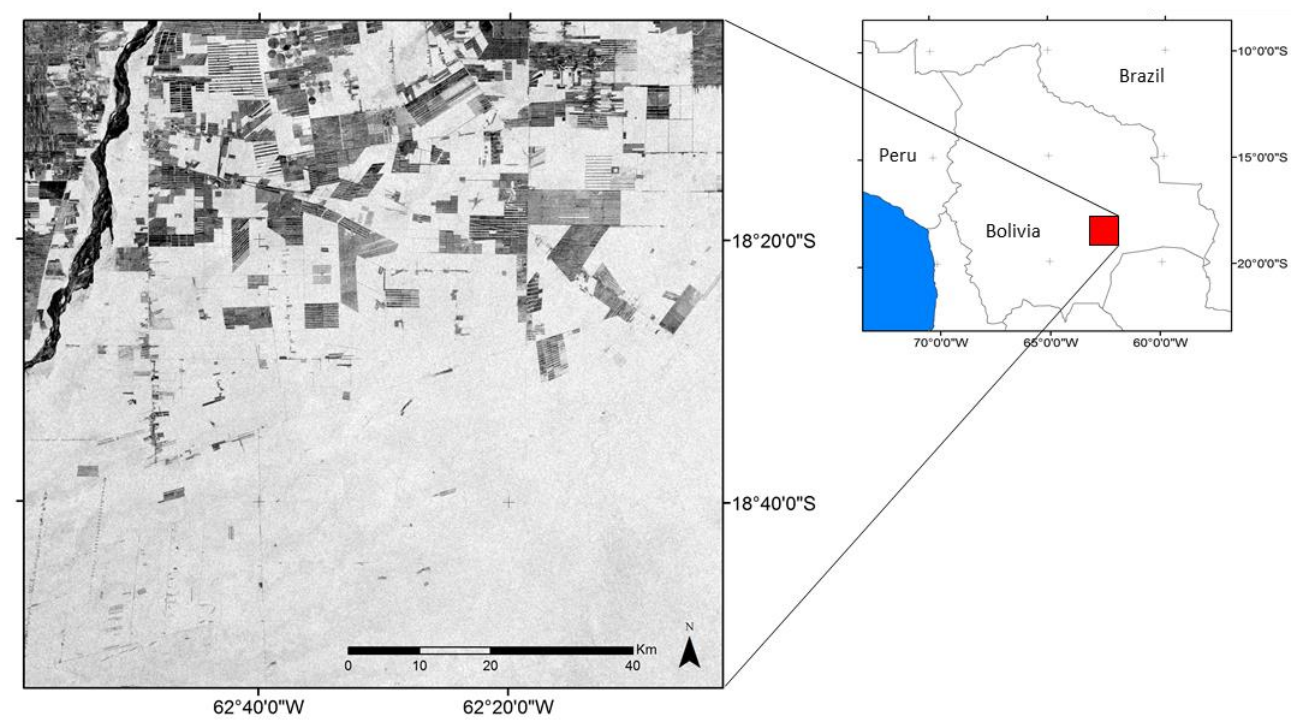

Figure 6.1: The dry tropical forest study site located in the province of Santa Cruz, Bolivia. The large area deforested patches that are the result from logging activities over the past decade are clearly visible. The base image is a Sentinel-1 VV-polarised image acquired at 2016-08-26.

\subsection{Data and methods}

An overview of the methods used in this study is shown in Figure 6.2. We acquired Sentinel-1 VV-polarised C-band SAR, ALOS-2 PALSAR-2 HVpolarised L-band SAR and Landsat (7 and 8) NDVI time series data for the two year period between 2014-10-01 and 2016-09-30; corresponding to the first two years of available Sentinel-1 images. Data from the first year was used to derive training information (training period), and data from the second year was used to detect new deforestation events (monitoring period). We first preprocessed the individual Sentinel-1 VV, ALOS-2 PALSAR-2 HV and Landsat NDVI time series (Section 6.3.1.1 - 6.3.1.3), including time series coregistration (Section 6.3.1.4). Next, a digitized forest benchmark map was used to mask out all non-forest areas at the beginning of our monitoring period (Section 6.3.2), before we applied spatial normalization to reduce dry forest seasonality in the individual time series (Section 6.3.3). We used a probabilistic approach to combine the spatially normalised time series and to detect deforestation in NRT (Section 6.3.4). We emulated a NRT scenario for the monitoring period (Section 6.3.5), and assessed the spatial and temporal accuracy of detected deforestation (Section 6.3.6). We compared the singlesensor results with multi-sensor results. 


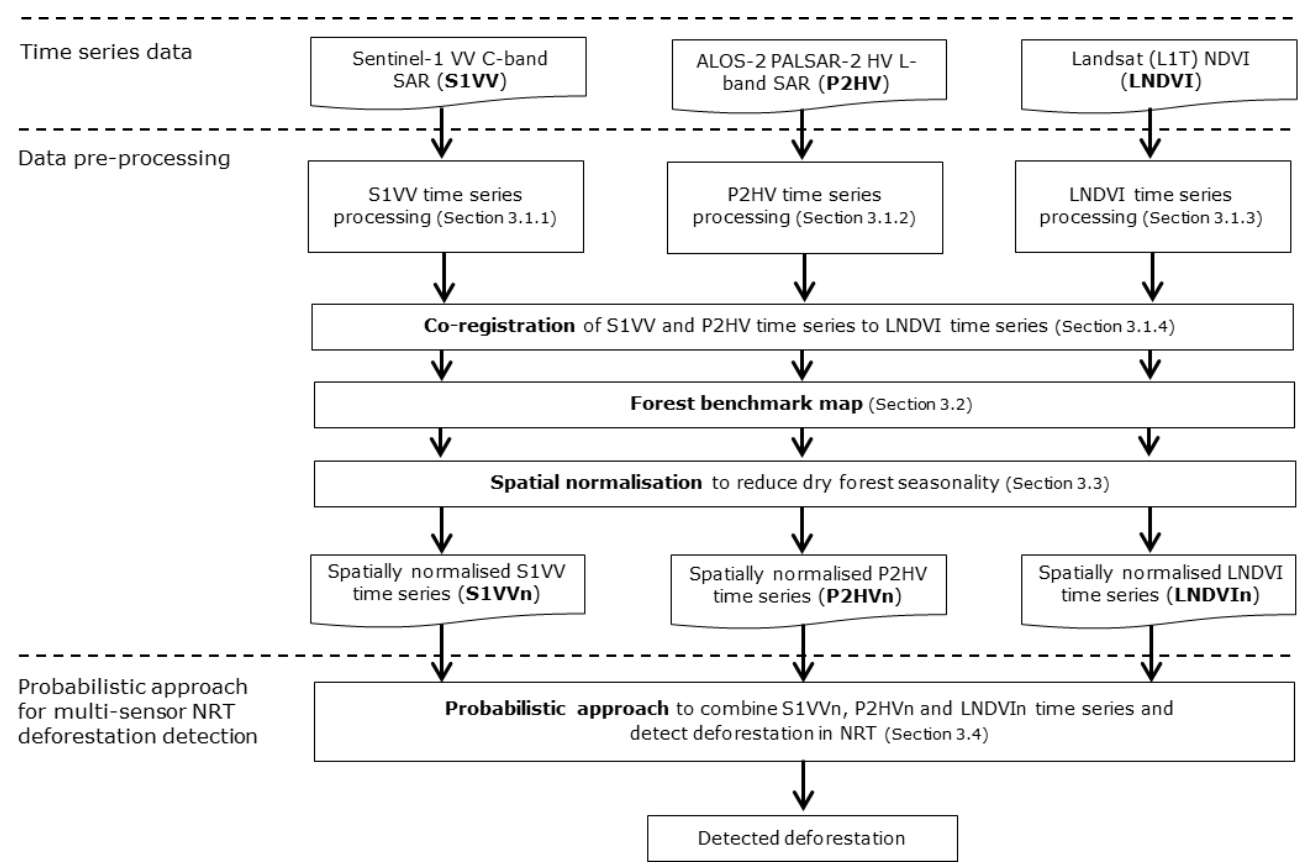

Figure 6.2: Flowchart used in this study to process and combine time series of Sentinel-1 VV-polarised C-band SAR, ALOS-2 PALSAR-2 HV-polarised L-band SAR and Landsat NDVI for NRT deforestation detection.

\subsubsection{Satellite image processing}

\subsubsection{Sentinel-1 VV-polarised C-band SAR}

We obtained Sentinel-1 VV-polarised (S1VV) images acquired in Interferometric Wide Swath (IW, $250 \mathrm{~km}$ swath width) from the Sentinel science hub (https://scihub.copernicus.eu/) for the period between 2014-10-01 and 2016-09-30. The Sentinel-1A C-band SAR instrument operates at $5.3 \mathrm{~cm}$ wavelength (Torres et al. 2012). In total, 162 single-polarised (VV) and 9 dualpolarised (VV and VH) acquisitions were retrieved. The images were acquired in ascending and descending mode with an incidence angle of $39^{\circ}\left( \pm 0.5^{\circ}\right)$ and provided in Level-1 Ground Range Detected (GRD) format. We used the VVpolarised images to build a time series (S1VV). Because of few dual-polarised acquisitions (VV and $\mathrm{VH}$ ) we did not consider the $\mathrm{VH}$ polarisation for this study.

Sentinel-1 time series processing consisted of five steps: (i) individual image pre-processing and geocoding, (ii) quality control, (iii) mosaicking, (iv) multi-temporal filtering, (v) and co-registration to Landsat. Firstly, we preprocessed each image individually using the Gamma software package (Wegmüller et al. 2016). Pre-processing consisted of data import and format 
conversion, multi-looking, radiometric calibration, topographic normalization and geocoding to $30 \mathrm{~m}$ pixel resolution (WGS84, UTM 20S) using the $30 \mathrm{~m}$ SRTM DEM (https://lta.cr.usgs.gov/SRTM1Arc). The outputs were geocoded and topographically normalised gamma-nought backscatter images at $30 \mathrm{~m}$ resolution. Secondly, we removed corrupted images and images with geolocation accuracy smaller than 0.5 pixels. In total, we removed 13 images. The resulting average geocoding accuracy was smaller than 0.2 pixels. This reflects an internally well co-registered S1VV time series. Thirdly, we mosaicked all images acquired at the same date. The pre-processed and mosaicked S1VV backscatter time series consists of 98 images. Fourthly, we applied adaptive multi-temporal SAR filtering (Quegan \& Yu 2001) to the preprocessed and mosaicked time series. A measured increase of the equivalent number of looks indicated a clear reduction of SAR speckle in the data. Fifthly, we co-registered the S1VV time series to Landsat images described in Section 6.3.1.4. The number of per-pixel observations averages 57 observations $(\sim 2.4$ obs. /month) and ranges between 47 ( 1.9 obs. /month) and 98 observations ( $4.1 \mathrm{obs}$. /month) for the two year period (Figure $6.3 \mathrm{a})$. The observations were distributed regularly over time. Due to overlapping of ascending and descending frames, the eastern part of the study area has a higher temporal coverage (Figure 6.3a).

\subsubsection{ALOS-2 PALSAR-2 HV-polarised L-band SAR}

ALOS-2 PALSAR-2 HV-polarised (P2HV) images acquired in Fine Beam Dual (FBD, 70 swath width) mode were obtained from the ALOS-2 data archive (https://auig2.jaxa.jp/ips/home) for the period between 2014-10-01 and 2016-0930. In total, 47 FBD images were obtained in Level 1.1 format. The images were acquired in ascending and descending mode with an incidence angle of $34.3^{\circ}$. We chose the HV polarization as ALOS-2 PALSAR-2 metric (P2HV). The higher capacity to detect forest cover change in L-band HV-polarised images when compared to $\mathrm{HH}$-polarised images is well recognised and has been demonstrated by multiple studies (Motohka et al. 2014; Ryan et al. 2012; Thapa et al. 2013; Joshi et al. 2016; Shimada et al. 2014). Time series processing of P2HV was as the processing of S1VV described in Section 6.3.1.2. We removed corrupted images and images with geolocation accuracy smaller than 0.5 pixels. In total, two images were removed. The geocoding accuracy was below 0.4 pixels for all images. This reflects an internally well co-registered P2HV time series. The pre-processed and mosaicked P2HV time series consists of 15 images. Co-registration to Landsat images is described in Section 6.3.4. The number of per-pixel P2HV observations averages 8 observations $(\sim 0.3$ obs. /month) and ranges between $4(\sim 0.15$ obs. /month) and 15 observations $(\sim 0.6$ obs. /month) (Figure 6.3b) for the two year period. 


\subsubsection{Landsat NDVI}

We obtained Landsat NDVI (LNDVI) products from the United State of America's Geological Survey (USGS) Landsat Surface Reflectance (SR) Climate Data Records (CDR) (http://landsat.usgs.gov/CDR_LSR.php) for the period between 2014-10-01 and 2016-09-30. The NDVI products are atmospherically and geometrically corrected and were derived from Level 1 Terrain corrected (L1T) Landsat-7/ETM+ and Landsat-8/OLI images. In total, 63 images (WGS84, UTM 20S) were acquired. We masked cloud and cloud shadows for each individual image using the CFmask cloud mask product which is distributed with the Landsat SR CDR data. We assumed the LNDVI time series to be internally well geocoded. The number of cloud-free LNDVI observations averages 30 observations ( 1.25 obs. /month) and ranges between 13 ( 0.5 obs. /month) and 44 observations ( 1.8 obs. /month) for the two year period (Figure 6.3c).

\subsubsection{Co-registration}

A precisely co-registered and integrated satellite image time series consisting of S1VV, P2HV and LNDVI images is crucial, in order to apply any SAR-optical algorithm. We used the GAMMA software package (Werner \& Strozzi 2000) to co-register the S1VV and P2HV time series to the LNDVI time series. Co-registration included an automated cross-correlation between the source image (S1VV / P2HV) and the reference target image (LNDVI), using improved least-squares polynomial fitting. The cross-correlation resulted in range and azimuth offset polynomial of $4^{\text {th }}$ order. The offset polynomials were subsequently used to co-register the source images to the reference image (Werner \& Strozzi 2000). Co-registration results indicated a sub-pixel accuracy of smaller than 0.01 pixels for both, S1VV and P2HV. For the multi-sensor S1VV, P2HV and LNDVI time series, the number of valid per-pixel observations averages 96 observations ( 4 obs./month) and ranges from 65 ( $\sim 2.7 \mathrm{obs} . / \mathrm{month})$ to $147 \mathrm{observations}(\sim 6.1 \mathrm{obs} . / \mathrm{month})$ for the two year period (Figure 6.3d).

\subsubsection{Benchmark forest mask}

We divided the two years period of available and overlapping S1VV, P2HV and LNDVI time series into a one year training period (2014-10-01 - 2015-0930 ) and a one year monitoring period (2015-10-01 - 2016-09-30). We used data from the training period to parametrize the probabilistic approach that we used to combine the S1VV, P2HV and LNDVI time series and to detect deforestation in NRT during the monitoring period (Section 6.3.4). To validate the detected deforestation events, reference deforestation information are required for the monitoring period. As for many tropical forest areas, reliable reference data for forest cover and forest cover change are not available. High to medium spatial 
resolution satellite data are commonly used to facilitate the derivation of validation data (Dutrieux et al. 2015; Hamunyela, Verbesselt, Bruin, et al. 2016; Cohen et al. 2010; Zhu \& Woodcock 2014b; Hansen et al. 2016).

We used the available S1VV, P2HV and LNDVI images complemented by high spatial resolution imagery available in Google Earth (https://www.google.com/earth/) and Bing Maps (http://www. bing.com/maps/) to derive reference information through visual image interpretation. First, we generated a forest benchmark mask representing all forested pixels at the beginning of the monitoring period. We only considered pixels that were entirely covered with forest. The forest strata accounts for $77.5 \%\left(7749.75 \mathrm{~km}^{2}\right)$ and the non-forest strata for $22.5 \%\left(2250.25 \mathrm{~km}^{2}\right)$. Based on the forest benchmark mask, we generated a deforestation mask for the monitoring period consisting of two strata: deforestation and stable forest. Deforestation was defined as loss of forest cover that is visually visible from the satellite images and the complementary high resolution maps. Stable forest areas represent all forest pixels that did not change during the monitoring period. The deforestation strata accounted for $1.03 \%\left(79.5 \mathrm{~km}^{2}\right)$ of the benchmark forest area, whereas the stable forest area accounted for the remaining $98.97 \%$ $\left(7670.25 \mathrm{~km}^{2}\right)$. 
(a) S1VV [min:47; mean:57; max:98]

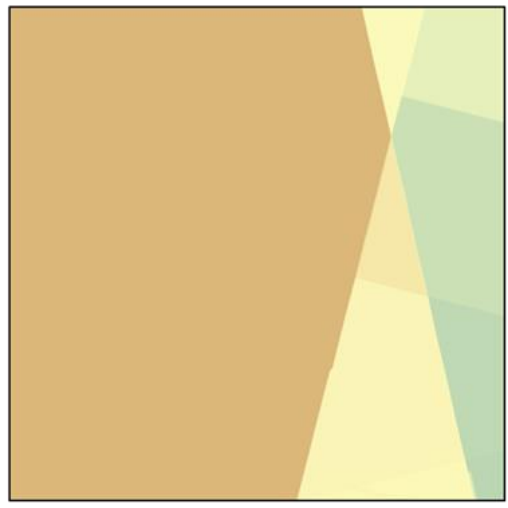

(c) LNDVI [min:13; mean: 30; max:44]

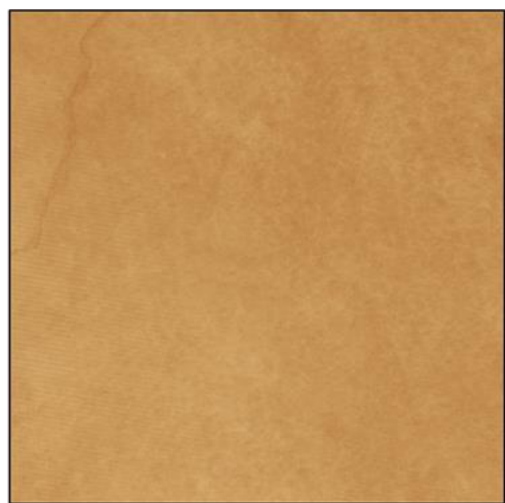

(b) P2HV [min:4; mean: 8; max:15]

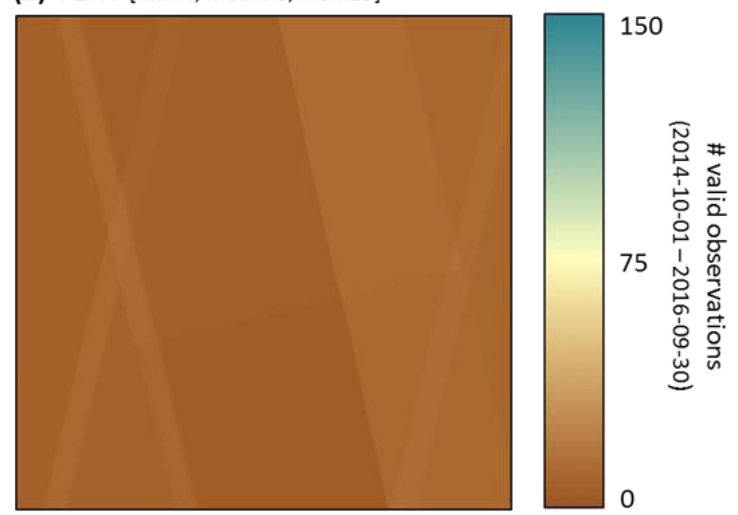

(d) Multi-sensor [min:65; mean:96; max:147]

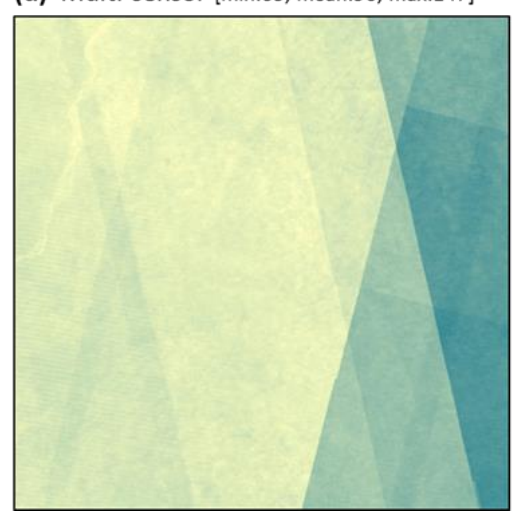

Figure 6.3: The number of valid per-pixel observations for Sentinel-1 VV (S1VV, a), ALOS-2 PALSAR-2 HV (P2HV, b), Landsat NDVI (LNDVI, c), and the multi-sensor time series (d) for the two year period between 2014-10-01 and 2016-09-30. The minimum, mean and maximum number of available per-pixel observations is given for each of the time series.

\subsubsection{Spatial normalization to reduce dry forest seasonality}

We used spatial normalization to reduce dry forest seasonality in the S1VV, P2HV and LNDVI time series whereby we built upon the method of Hamunyela, Verbesselt \& Herold (2016). Pixels were spatially normalised by subtracting the $95^{\text {th }}$ percentile, P95, of the distribution of pixel within their spatial neighbourhood. It is based on the assumption that the upper tail of the distribution (corresponding to $\mathrm{P}_{95}$ ) of pixels within the spatial neighbourhood represents forest pixels (Hamunyela, Verbesselt \& Herold 2016). To spatially normalise a pixel, we subtract $\mathrm{P}_{95}$ from the pixel instead of dividing the pixel by $\mathrm{P}_{95}$ as proposed by Hamunyela, Verbesselt \& Herold (2016). This ensures that the difference between $\mathrm{P}_{95}$ and the pixel to be normalised results in the same 
normalised pixel value for varying $\mathrm{P}_{95}$. The $2 \mathrm{~dB}$ difference between $-14 \mathrm{~dB}$ (corresponding to $\mathrm{P}_{95}$ ) and $-16 \mathrm{~dB}$ (corresponding to the pixel to be normalised) and the $2 \mathrm{~dB}$ difference between $-15 \mathrm{~dB}$ and $-17 \mathrm{~dB}$, for example, results in the same normalised pixel value of $-2 \mathrm{~dB}$. This allows the robust application to nonnormalised metrics with open value scales such as the S1VV and P2HV SAR backscatter images.

A forest benchmark mask is required so that (i) non-forest land cover types such as agriculture do not affect $\mathrm{P}_{95}$, and (ii) that the seasonality for forest pixel can be captured by $\mathrm{P}_{95}$. We used the benchmark forest mask (Section 6.3.2) to mask out all non-forest pixels in the S1VV, P2HV and LNDVI time series images. Further, it is crucial that the spatial window used to calculate $\mathrm{P}_{95}$ covers forest areas. Otherwise deforested areas are erroneously normalised with $\mathrm{P}_{95}$ that was calculated based on deforested pixels instead of forest pixels. A large spatial window is required at our study site, because we deal with large logging operations and forest patches being surrounded by already deforested land. As our study site is relatively small and is characterised by homogeneous dry forest areas, we used a global $\mathrm{P}_{95}$, calculated for the entire study area. We assumed that environmental effects such as droughts are regional, affecting the whole of our study area in the same manner (Hamunyela, Verbesselt \& Herold 2016). The spatially normalised S1VV, P2HV and LNDVI time series are hereafter referred to as S1VVn, P2HVn and LNDVIn, respectively.

\subsubsection{Probabilistic approach for multi-sensor NRT deforestation detection}

Building upon the approach of Reiche, et al. (2015), we present a probabilistic approach to combine the spatially normalized S1VVn, P2HVn (both SAR) and LNDVIn (optical) time series and to detect deforestation in NRT. For method parametrisation, we first derived sensor specific forest (F) and non-forest (NF) probability density functions ( $\mathrm{pdfs}$ ) using data from the training period (Section 6.3.4.1). Figure 6.4 gives a schematic overview the probabilistic approach. We considered a NRT scenario with past $(\mathrm{t}-1)$, current $(\mathrm{t})$ and future observations $(\mathrm{t}+1)$, with multiple observations possible at the same observation date. First (Section 6.3.4.2), once a new observation of either of the input time series was available $(\mathrm{t}=$ current $)$ it was converted to the conditional NF probability $\left(\mathrm{s}^{\mathrm{NF}}\right)$ using the sensor specific $\mathrm{F}$ and $\mathrm{NF}$ pdf. The derived conditional NF probability was added to the combined time series of conditional NF probabilities derived from the previous S1VVn, P2HVn and LNDVIn time series observations ( $\mathrm{t}-\mathrm{i}$ ). Second (Section 6.3.4.3), we flagged a potential deforestation event in the case that the conditional NF probability was larger than 0.5. We calculated the probability of deforestation using iterative Bayesian updating. Future observation $(t+i)$ were used to update the probability of deforestation in order to confirm or reject the flagged deforestation event. 


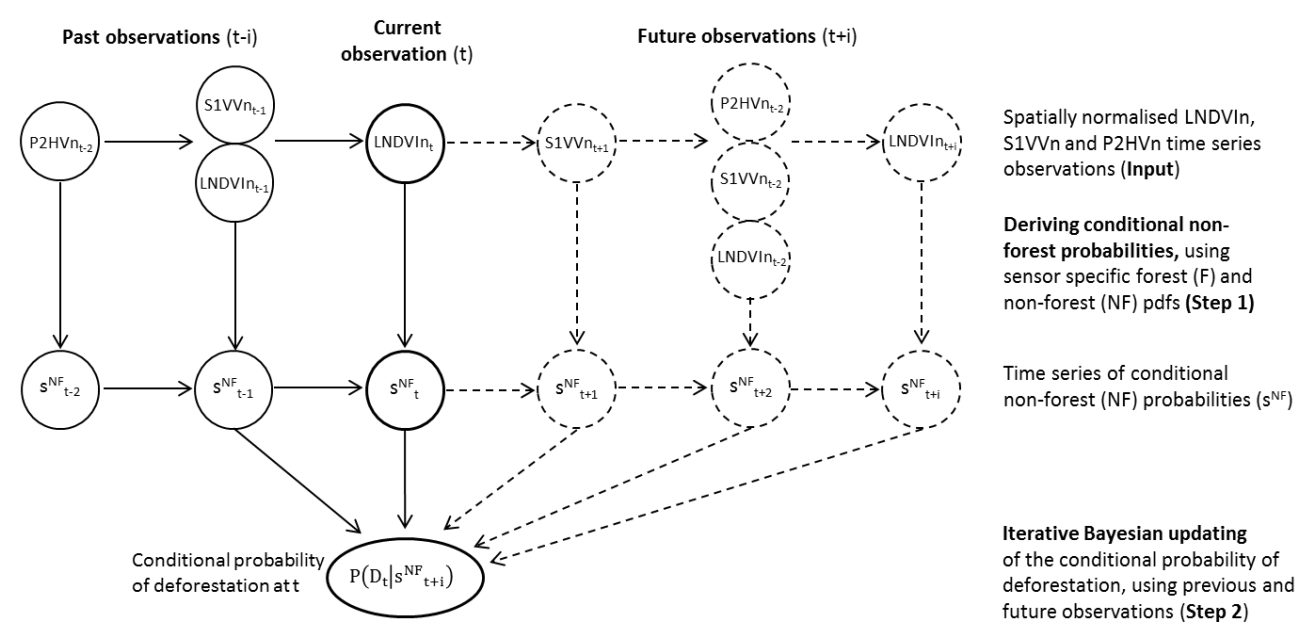

Figure 6.4: Probabilistic approach used to combine time series of spatially normalized Sentinel-1 VV (S1VVn), ALOS-2 PALSAR-2 HV (P2HVn) and Landsat NDVI (LNDVIn) observations and to detect deforestation in NRT.

\subsubsection{Deriving sensor specific forest and non-forest pdfs from training data}

We derived sensor specific probability density functions (pdfs) for forest (F) and non-forest (NF), separately for S1VVn, P2HVn and LNDVIn. We selected ten equally sized stable $\mathrm{F}$ and $\mathrm{NF}$ training areas $(\sim 25$ ha each) for the training period through visual image interpretation, whereby we followed the method described in Section 6.3.2. We used all observations available for the training period and covering the $\mathrm{F}$ and $\mathrm{NF}$ training areas to derived sensor specific $\mathrm{F}$ and NF distributions. We fitted a Gaussian model separately to the F and NF distributions. Maximum likelihood fitting with an iterative optimization method was used (Venables \& Ripley 2002). We evaluated the F/NF separability for S1VVn, P2HVn and LNDVIn using the normalized JeffriesMatusita distance (JM) (Laliberte et al. 2012). JM has a finite dynamic range between 0 (inseparable) and 2 (separable). We compared the F/NF separability obtained for S1VVn, P2HVn and LNDVIn with results obtained for S1VV, P2HV and LNDVI.

\subsubsection{Deriving and combining time series of conditional NF probabilities}

Using the corresponding sensor specific $\mathrm{F}$ and NF pdfs (Section 6.3.4.1), we estimated the conditional NF probability for individual time series observations. Following Bayes' theorem, we calculated the conditional NF probabilities for S1VVn, P2HVn and LNDVIn observation: $\mathrm{P}(\mathrm{NF} \mid \mathrm{S} 1 \mathrm{VVn})$, $\mathrm{P}(\mathrm{NF} \mid \mathrm{P} 2 \mathrm{HVn})$ and $\mathrm{P}(\mathrm{NF} \mid \mathrm{LNDVIn})$. For example, the conditional NF probability for a S1VVn observation was calculated as: 
$P(N F \mid S 1 V V n)=\frac{p(S 1 V V n \mid N F)}{p(S 1 V V n \mid N F)+p(S 1 V V n \mid F)}$

with $\mathrm{p}(\mathrm{S} 1 \mathrm{VVn} \mid \mathrm{F})$ and $\mathrm{p}(\mathrm{S} 1 \mathrm{VVn} \mid \mathrm{NF})$ being the probability of the S1VVn observation given the presence of $\mathrm{F}$ and $\mathrm{NF}$, respectively. $\mathrm{p}(\mathrm{S} 1 \mathrm{VVn} \mid \mathrm{F})$ and $\mathrm{p}(\mathrm{S} 1 \mathrm{VVn} \mid \mathrm{NF})$ were derived from the sensor specific S1VVn F and NF pdfs. We used a block weighting function that modifies $\mathrm{P}(\mathrm{NF} \mid \mathrm{S} 1 \mathrm{VVn})$ at 0.1 and 0.9 to avoid extreme probabilities (Reiche, de Bruin, et al. 2015).

Next, we combined the conditional NF probabilities estimated for the individual S1VVn, P2HVn and LNDVIn, ( $(\mathrm{NF} \mid \mathrm{S} 1 \mathrm{VVn}), \mathrm{P}(\mathrm{NF} \mid \mathrm{P} 2 \mathrm{HVn})$ and ( $\mathrm{P}(\mathrm{NF} \mid \mathrm{LNDVIn})$ ) observations to create a new time series of conditional $\mathrm{NF}$ probabilities, $\mathrm{s}^{\mathrm{NF}}$. In case that multiple observations from different sensors were acquired at the same observation date, we computed $\mathrm{s}^{\mathrm{NF}}$ jointly from the multiple observations using Bayesian updating. For example, in case of a S1VVn and LNDVIn observation acquired at the same date, $\mathrm{s}^{\mathrm{NF}}$ was calculated as:

$\mathrm{S}^{\mathrm{NF}} \mid \mathrm{S} 1$ VVn, LNDVIn $=\frac{\mathrm{P}(\mathrm{NF} \mid \mathrm{S} 1 \mathrm{VVn}) * \mathrm{P}(\mathrm{NF} \mid \mathrm{LNDVIn})}{(\mathrm{P}(\mathrm{NF} \mid \mathrm{S} 1 \text { VVn }) * \mathrm{P}(\mathrm{NF} \mid \mathrm{LNDVIn})+(1-\mathrm{P}(\mathrm{NF} \mid \mathrm{S} 1 \text { VVn })) *(1-\mathrm{P}(\mathrm{NF} \mid \mathrm{LNDVIn})))}$

\subsubsection{Iterative Bayesian updating to detect deforestation}

We checked each new observation $(t=$ current $)$ if it was potentially deforested. It was flagged to be potentially deforested in the case that the conditional NF probability $\left(\mathrm{s}_{\mathrm{NF}}\right)$ exceeded 0.5. For a flagged observation, the conditional probability of deforestation was computed by iterative Bayesian updating, using the previous observation $(t-1)$, the current observation $(t)$, as well as upcoming observations $(t+i)$ :

$P\left(D_{t} \mid s^{N F}{ }_{t+i}\right)=\frac{P\left(s^{N F}{ }_{t+i} \mid D\right) P\left(D_{t} \mid s^{N F}{ }_{t+i-1}\right)}{P\left(s^{N F}{ }_{t+i}\right)}$

with $\mathrm{P}\left(\mathrm{D}_{\mathrm{t}} \mid \mathrm{s}^{\mathrm{NF}} \mathrm{t+ \textrm {i }}\right)$ being the posterior, denoting the conditional probability of deforestation at $t\left(D_{t}\right)$ given $\mathrm{s}^{\mathrm{NF}} \mathrm{t}_{+\mathrm{i}}$ as new evidence. $\mathrm{P}\left(\mathrm{s}^{\mathrm{NF}} \mathrm{t+i}\right)$ refers to the total probability of the signal $\mathrm{s}^{\mathrm{NF}} \mathrm{t+i}$. $\mathrm{i}$ refers to the $\mathrm{i}$-th future observation that can take values between 0 and $n$.

For the initial step ( $\mathrm{i}=0=$ current), $\mathrm{P}\left(\mathrm{D}_{\mathrm{t}} \mid \mathrm{s}^{\mathrm{NF}} \mathrm{t}\right)$ was calculated using the conditional NF probability at the current step $\left(\mathrm{s}_{\mathrm{NF}}\right)$ as new evidence, and the conditional NF probability at the previous time step $\left(\mathrm{s}^{\mathrm{NF}_{\mathrm{t}-1}}\right)$ as the prior probability. With the availability of future observation in subsequent steps ( $>$ 0 ), the conditional probability of deforestation $\left(D_{t}\right)$ was repeatedly updated. Hereby, upcoming observations $\left(\mathrm{s}_{\mathrm{NF}}\right)$ were used as new evidence (Reiche, de Bruin, et al. 2015). Deforestation events were confirmed if the conditional probability of deforestation exceeded a defined threshold $\mathrm{X}$. The $\mathrm{X}$ threshold 
value can range between 0 and $<1$. Choosing high $\mathrm{X}$ threshold values increase the confidence to confirm deforestation events, because more observations are used to reach a high probability of deforestation, and, thus to confirm the change. When compared to lower $\mathrm{X}$ threshold values, however, events are more likely to be confirmed later. In case the conditional probability of deforestation decreased below 0.5 when using future observation, we considered a falsely indicated deforestation event. In this case we unflagged the observation as being potentially deforested, and continued monitoring (Reiche, de Bruin, et al. 2015).

\subsubsection{Detecting deforestation in an emulated NRT scenario}

We emulated a NRT scenario for the monitoring period to validate the proposed method with respect to its NRT deforestation detection performance. We added the individual S1VVn, P2HVn and LNDVIn observations chronologically to the multi-sensor time series from the start of the monitoring period onwards (2015-10-01). We applied the probabilistic approach described in the previous Section 6.3.4 to each newly added observation with subsequent observations being considered as future observations. We analysed the results for increasing $X$ threshold values from $X=0.5$ to $X=0.975$ in steps of 0.025 . We compared the multi-sensor results obtained for the combined S1VVn, P2HVn and LNDVIn time series with single-sensor results.

\subsubsection{Estimating the spatial and temporal accuracy}

We estimated the spatial and temporal accuracy for the deforestation maps. We generated 1877 sample points using probability sampling consistent with Stehman (2012) and Olofsson et al. (2014). To address the small proportion of deforestation in the monitoring period (1.03\%), which is typical for land changes, we followed Olofsson et al. (2014) and allocated 200 sample points to the deforestation stratum. The remaining 1677 samples were allocated to the stable forest stratum. This allocation ensured a reliable estimation of both, the producer's and user's accuracy of the deforestation class. We calculated the area adjusted overall accuracy (OA), producer's accuracy (PA; 1- omission error) and user's accuracy (UA; 1 - commission error). Due to the very small area proportion of the deforestation class, the OA is mainly driven by the UA and PA of the dominating stable forest class and is not a good measure to assess the method performance to detect deforestation. The UA and PA of the deforestation class are more useful for the comparison of singleversus multi-sensor approaches.

To estimate the temporal accuracy, we calculated the mean time lag of confirmed deforestation events (MTL). In addition, we calculated the mean time lag of the time at which the confirmed deforestation events were initially flagged (MTLF). To calculate MTL and MTLF, we retrieved reference 
information from the deforestation sample. For each of the 200 sample points, we documented the date at which the deforestation was visible for the first time $\left(\mathrm{T}_{\mathrm{ref}}\right)$ by systematically tracing the images in the multi-sensor S1VV, $\mathrm{P} 2 \mathrm{HV}$ and LNDVI time series. The approach is consistent with approaches recommended or used in previous studies (Cohen et al. 2010; Devries, Verbesselt, et al. 2015; Hansen et al. 2016; Zhu \& Woodcock 2014b)

Commonly $\mathrm{T}_{\text {ref }}$ is used as reference data to calculate the temporal accuracy or temporal detection delay (Zhu \& Woodcock 2014a; Pratihast et al. 2016; Hansen et al. 2016). Tref, however, does not represent the "true" date of the deforestation event, but rather provides a reference date relative to the satellite time series observations itself. The "true" date instead occurred sometime between the image in which the deforestation event is first visible $\left(\mathrm{T}_{\mathrm{ref}}\right)$ and the previous image in the time series ( $\left.\mathrm{T}_{\text {ref_previous}}\right)$. The time span between $\mathrm{T}_{\text {ref }}$ and $\mathrm{T}_{\text {ref_previous }}$ depends on the observation density and is varying across space (see Figure 6.3d). For the 200 deforestation sample points, the

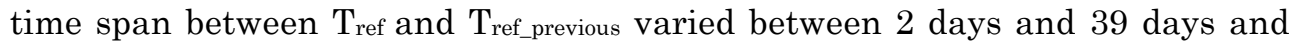
averages 13 days (Figure 6.5). The time span describes the imprecision of $\mathrm{T}_{\text {ref. }}$ Accordingly, the average imprecision was 13 days. To reduce the imprecision of $\mathrm{T}_{\text {ref }}$ in the absence of any further information on when the change truly occurred within the time span, we calculated an adjusted reference time, $\mathrm{T}_{\text {ref_adjusted, }}$ as the date right in-between $\mathrm{T}_{\text {ref }}$ and $\mathrm{T}_{\text {ref_previous: }}$ :

$$
\mathrm{T}_{\text {ref_adjusted }}=\mathrm{T}_{\text {ref }}-\frac{\left(\mathrm{T}_{\text {ref }}-\mathrm{T}_{\text {ref_previous }}\right)}{2}
$$

This reduced the average imprecision by fifty percent to \pm 6 days. Next, we calculated the time lag of detected deforestation events, TL, by calculating the time span between Tref_adjusted and the date at which a deforestation event was confirmed by our algorithm (T). We treated detected deforestation events that have been flagged $\left(\mathrm{T}_{\mathrm{F}}\right)$ earlier than first visible in the multi-sensor time series $\left(\mathrm{T}_{\mathrm{ref}}\right)$ as commission error and exclude them from the calculation:

$$
\mathrm{TL}=\mathrm{T}\left(\mathrm{T}_{\mathrm{F}}>\mathrm{T}_{\text {ref }}\right)-\mathrm{T}_{\text {ref_adjusted }}
$$

Analogously to TL, we calculate the time lag of the date at which the confirmed changes were first flagged, TLF:

$$
\mathrm{TL}_{\mathrm{F}}=\mathrm{T}_{\mathrm{F}}\left(\mathrm{T}_{\mathrm{F}}>\mathrm{T}_{\text {ref }}\right)-\mathrm{T}_{\text {ref_adjusted }}
$$

We calcualted MTL as the mean of TL for all correctly detected change pixels. We derived MTLF as the mean of TLF for all correctly detected change pixels. MTL and MTLF were given in days. It is important to analyse MTL and MTLF jointly with the OE, because MTL and MTLF are calculated based on correctly detected changes only. 


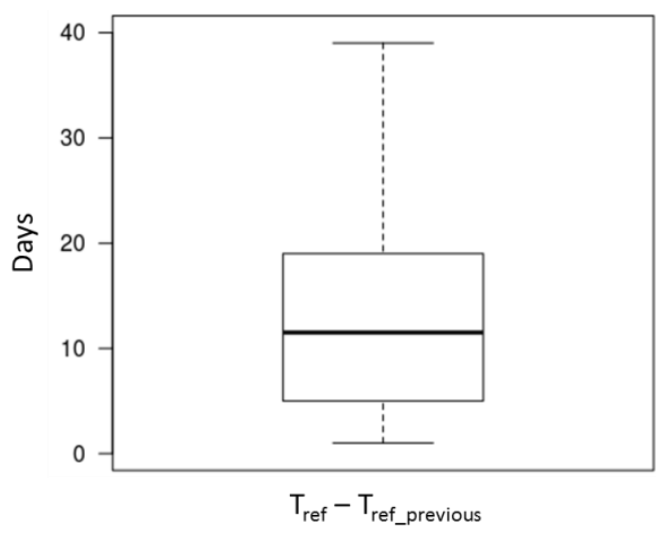

Figure 6.5: The time span between the image at which the deforestation event is first visible in the multi-sensor S1VV, P2HV and LNDVI time series ( $\mathrm{T}_{\text {ref }}$ ) and the previous image in the time series ( $\mathrm{T}_{\text {ref_previous }}$ ).

\subsection{Results}

\subsubsection{Spatial normalization and derived $\mathrm{F}$ and NF pdfs}

Figure 6.6 depicts the $\mathrm{F}$ and NF distributions overlaid with fitted pdfs separately for the original and spatially normalized time series: S1VV (a1) and S1VVn (b2), P2HV (b1) and P2HVn (b2), LNDVI (c1) and LNDVIn (c2). For the original time series we found LNDVI to have the weakest F/NF class separability ( $\mathrm{JM}=0.66)$ when compared to S1VV (JM=1.14) and P2HV (JM = 1.91). The bimodal LNDVI class distribution for $\mathrm{F}$ corresponds to wet and dry season observations, whereby the dry season observations largely overlap with the NF class. S1VV and P2HV show unimodal F and NF distributions. We found strongly increased F/NF separabilities for the spatial normalized S1VVn $(\mathrm{JM}=1.44), \mathrm{P} 2 \mathrm{HVn}(\mathrm{JM}=1.98)$ and LNDVIn ( $\mathrm{JM}=0.91)$ time series. The derived sensor specific pdfs for the spatially normalized time series were used for method parametrization. Figure 6.7 shows an example time series with the original (black dots) and spatially normalized (blue dots) observations for the two year period: S1VV and S1VVn (a), P2HV and P2HVn (b), LNDVI and LNDVIn (c). The pixel time series covers a stable dry forest that is deforested in January 2016. In addition to the time series covering the 2-year overlapping period, the LNDVI and LNDVIn time series are shown for a seven year period (2010 - 2016; Figure 6.7c1). The strong seasonality in photosynthetic activity, typical for dry forest, is clearly reflected in the original LNDVI time series (Figure 6.7c, c1). The original S1VV and P2HV SAR time series show a much weaker seasonal signal for stable dry forest. The seasonality was strongly reduced for the spatially normalized time series, in particular for LNDVIn. 
Original

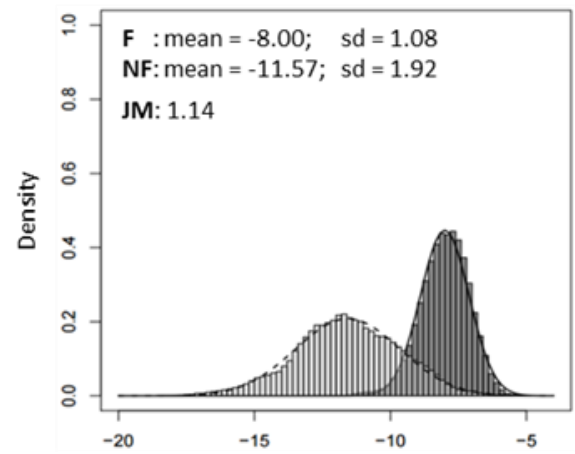

(a1) S1VV [dB]

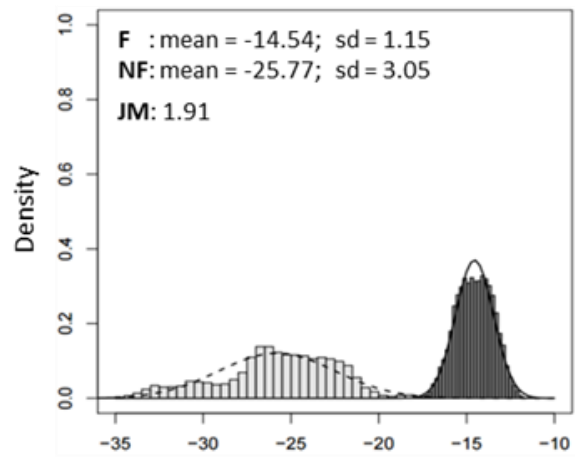

(b1) $\mathrm{P} 2 \mathrm{HV}[\mathrm{dB}]$

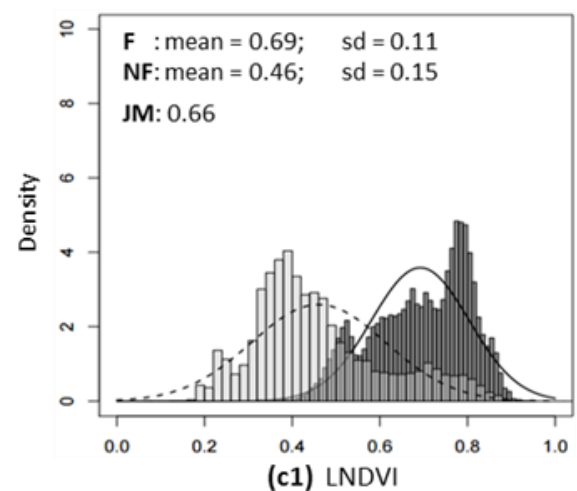

Spatially normalised

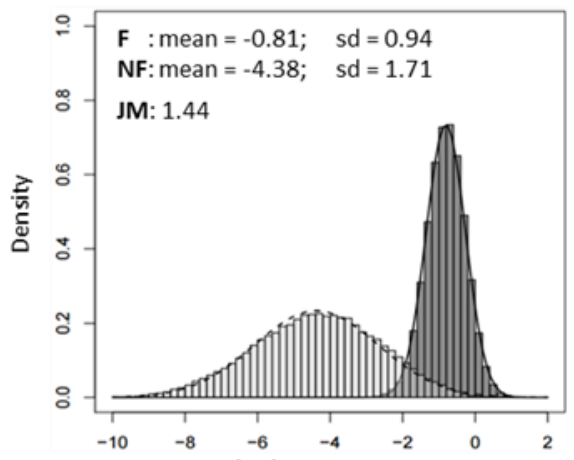

(a2) S1VVn [dB]

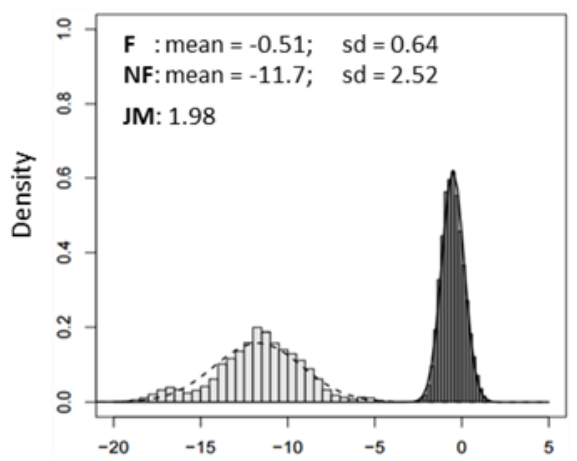

(b2) P2HVn [dB]

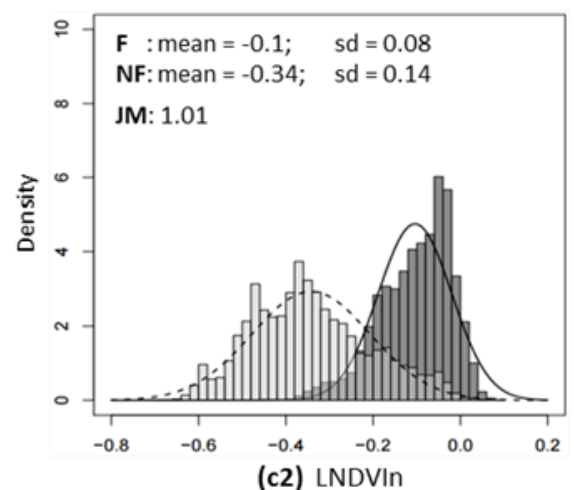

Figure 6.6. Forest (F) and non-forest (NF) distributions overlaid with probability density functions (pdfs) fitted separately for the original S1VV (a1), P2HV (b1) and LNDVI (c1) time series, and the spatially normalized S1VVn (a2), P2HVn (B2) and LNDVIn (a2) time series. Mean and standard deviation (sd) of the derived pdfs and the Jeffries-Matusita distance (JM) are given. JM ranges between 0 (inseparable) and 2 (separable). 


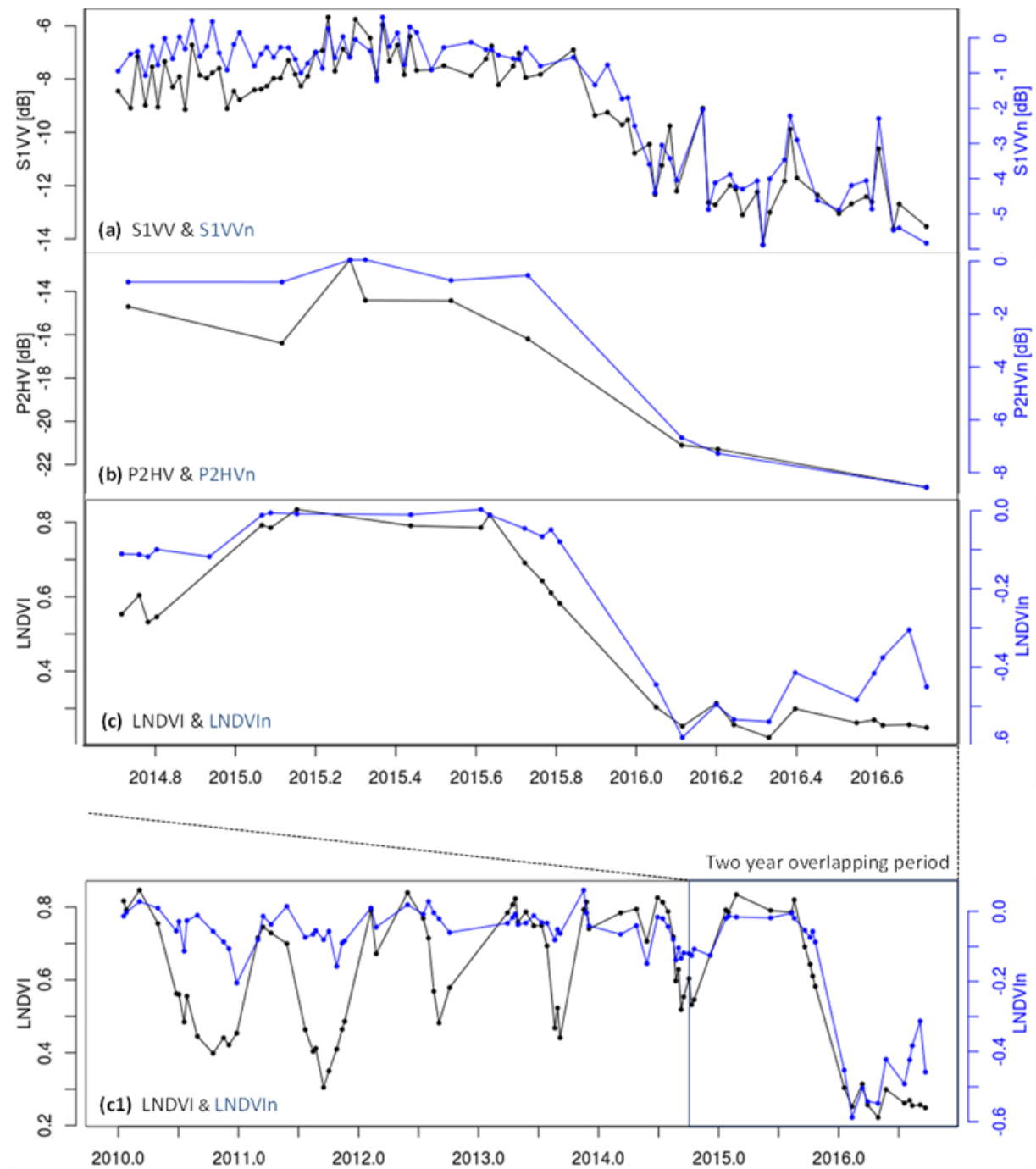

Figure 6.7: Example time series with the original (black dots) and spatially normalized (blue dots) observations: S1VV and S1VVn (a), P2HV and P2HVn (b), LNDVI and LNDVIn (c). In addition to the time series covering the 2-year overlapping period (a, b, c), the LNDVI and LNDVIn time series are shown for a seven year period $(2010$ - 2016; Figure 6.7c1).) This shows the annual seasonal dry forest pattern reflected in the LNDVI time series signal. When compared to the original LNDVI (c and c1) time series, the original S1VV (a) and P2HV (b) SAR time series show a weaker seasonality over stable dry forest. The pixel covers a stable dry forest that was deforested in January 2016. The time series are from the same pixel. 


\subsubsection{Detected deforestation}

Figure 6.8 illustrates a single pixel example with a deforestation event in January 2016, and shows the results for the single-sensor cases, S1VVn (a), P2HVn (b), LNDVIn (c) and the multi-sensor case (d). For the multi-sensor case the time series of conditional NF probabilities, $\mathrm{s}^{\mathrm{NF}}$, is shown in addition ( $\mathrm{d}$ bottom). The reference time at which the change was first visible in the satellite time series $\left(\mathrm{T}_{\mathrm{ref}}\right)$ is 2015(354) (day of year) and the adjusted reference time ( $\mathrm{T}_{\text {ref_adjusted }}$ ) was 2016(4). The adjusted reference time was used to calculate the mean time lag of confirmed changes (MTL), as well as the mean time lag of the time at which confirmed changes were first flagged (MTLF). For the multisensor case, the deforestation event was confirmed at $\mathrm{T}=2008(16)$, which is 12 days after it was flagged at $\mathrm{T}_{\mathrm{F}}=2008(04)$. When compared to multi-sensor case, the deforestation event was confirmed 6 days later with S1VVn, 25 days later for LNDVIn and 56 days later with P2HVn.

\subsubsection{Spatial and temporal accuracies for increasing $\mathrm{X}$ threshold}

The spatial accuracy (OA; PA and UA of the deforestation class) and temporal accuracy (MTL and MTLF) are shown in Figure 6.9 for increasing of $X$ threshold values, for the single-sensor cases, S1VVn (a) and LNDVIn (b), and multi-sensor case (c). Single-sensor results for the P2HVn are not presented. Because the $\mathrm{P} 2 \mathrm{HVn}$ observations available in the monitoring period were very limited, with some areas covered with one observation only, most deforestation events were not detected. A valid comparison with the other cases was therefore not possible. The P2HVn single pixel example depicted in Figure 6.7b and $6.8 \mathrm{~b}$ have above average observation availability. The results for S1VVn, LNDVIn, and the multi-sensor case can be summarized as follows.

For S1VVn (Figure 6.9a), we found increasing UA and decreasing PA of the deforestation class was obtained by increasing $\mathrm{X}$ threshold values. The UA/PA cross-over point occurred at $\mathrm{X}=0.85$. At the cross-over point, the UA and PA for the deforestation class were $85.9 \%$ and $82.7 \%$, respectively. The maximum OA $(99.7 \%)$ for S1VVn was also achieved at the UA/PA cross-over point. The UA and PA for the stable forest class (not shown in Figure 6.9a) were generally higher than the UA and PA for the deforestation class. For the $\mathrm{UA} / \mathrm{PA}$ cross-over point $(\mathrm{x}=0.85)$, UA and PA for the stable forest class were $99.8 \%$ and $99.0 \%$, respectively. The MTL increased for increasing $X$ threshold values from 21 days (for $\mathrm{X}=0.5$ ) to 58 days (for $\mathrm{X}=0.975$ ), and was 39 days at the UA/PA cross-over point. The MTLF was similar for all $X$ threshold values and was 16 days for the UA/PA cross-over point.

For LNDVIn (Figure 6.9b), spatial and temporal accuracies were generally lower when compared to those of S1VVn. We found an increasing UA and decreasing PA of the deforestation class for increasing $X$ threshold values. No UA/PA cross-over point was found due to a low UA. The maximum OA (99.1\%) 
was achieved at $\mathrm{X}=0.975$, and the corresponding UA and $\mathrm{PA}$ of the deforestation class were $51.5 \%$ and $69.5 \%$, respectively. The UA and PA for the stable forest class (not shown in Figure 6.9b) were generally higher than the UA and PA for the deforestation class. For $\mathrm{X}=0.975$, UA and PA for the stable forest class were $99.7 \%$ and $99.0 \%$, respectively. The MTL and MTLF increased for increasing $\mathrm{X}$ threshold values from 27 days (for $\mathrm{X}=0.5$ ) to 78 days (for $\mathrm{X}=$ 0.975). The MTLF was similar for all $X$ threshold values and was 18 days for $X=$ 0.975 .

The spatial and temporal accuracies for the multi-sensor case (Figure 6.9c) were generally higher when compared to those of single-sensor S1VVn, P2HVn and LNDVIn cases. For increasing $X$ threshold values, the UA of the deforestation class increased while the PA for the deforestation class decreased. The UA for deforestation class increased from 8.8\% (for $\mathrm{X}=0.5$ ) to $93.2 \%$ (for $\mathrm{X}$ $=0.975$ ), whereas the PA decreased from $100 \%$ (for $\mathrm{X}=0.5$ ) to $87.4 \%$ (for $\mathrm{X}=$ $0.975)$. The UA/PA cross-over point occurred at $X=0.925$, for which the UA and PA of the deforestation class were $88 \%$ and $88.9 \%$, respectively. The maximum OA $(99.8 \%)$ for the multi-sensor case was also found at the UA/PA cross-over point. For the UA/PA cross-over point $(\mathrm{X}=0.925)$, UA and PA for the stable forest class (not shown in Figure 6.9c) were 99.9\% and 99.0\%, respectively. The MTL increased for increasing $X$ threshold values from 13 days (for $X=0.5$ ) to 39 days (for $X=0.975$ ). At the UA/PA cross-over point, the MTL was 31 days. The MTLF was similar for all $X$ threshold values and was 11 days for the UA/PA cross-over point.

\subsubsection{Deforested area}

We choose a $X$ threshold value for which we achieved the lowest area bias to generate the final deforestation maps for S1VVn, LNDVIn and the multisensor case (Figure 6.10). For all three cases, the $\mathrm{X}$ threshold value with the lowest area bias also corresponds to the maximum OA. For S1VVn and the multi-sensor case this refers to the UA/PA cross-over point (Figure $6.9 \mathrm{~b}$ and 6.9c). We choose $X=0.975$ for LNDVIn, $X=0.85$ for S1VVn and $X=0.925$ for multi-sensor case. Different key user scenarios for selecting a $X$ threshold value are discussed in Section 6.5.1. The total deforested area for the one year monitoring period between 2015-10-01 and 2016-09-30 was estimated to be $67.8 \mathrm{~km}^{2}\left(\right.$ bias $\left.=2.8 \mathrm{~km}^{2}\right)$ for S1VVn, $56.7 \mathrm{~km}^{2}\left(\right.$ bias $\left.=22.6 \mathrm{~km}^{2}\right)$ for LNDVIn and $71 \mathrm{~km}^{2}\left(\right.$ bias $\left.=0.4 \mathrm{~km}^{2}\right)$ for the multi-sensor case. 

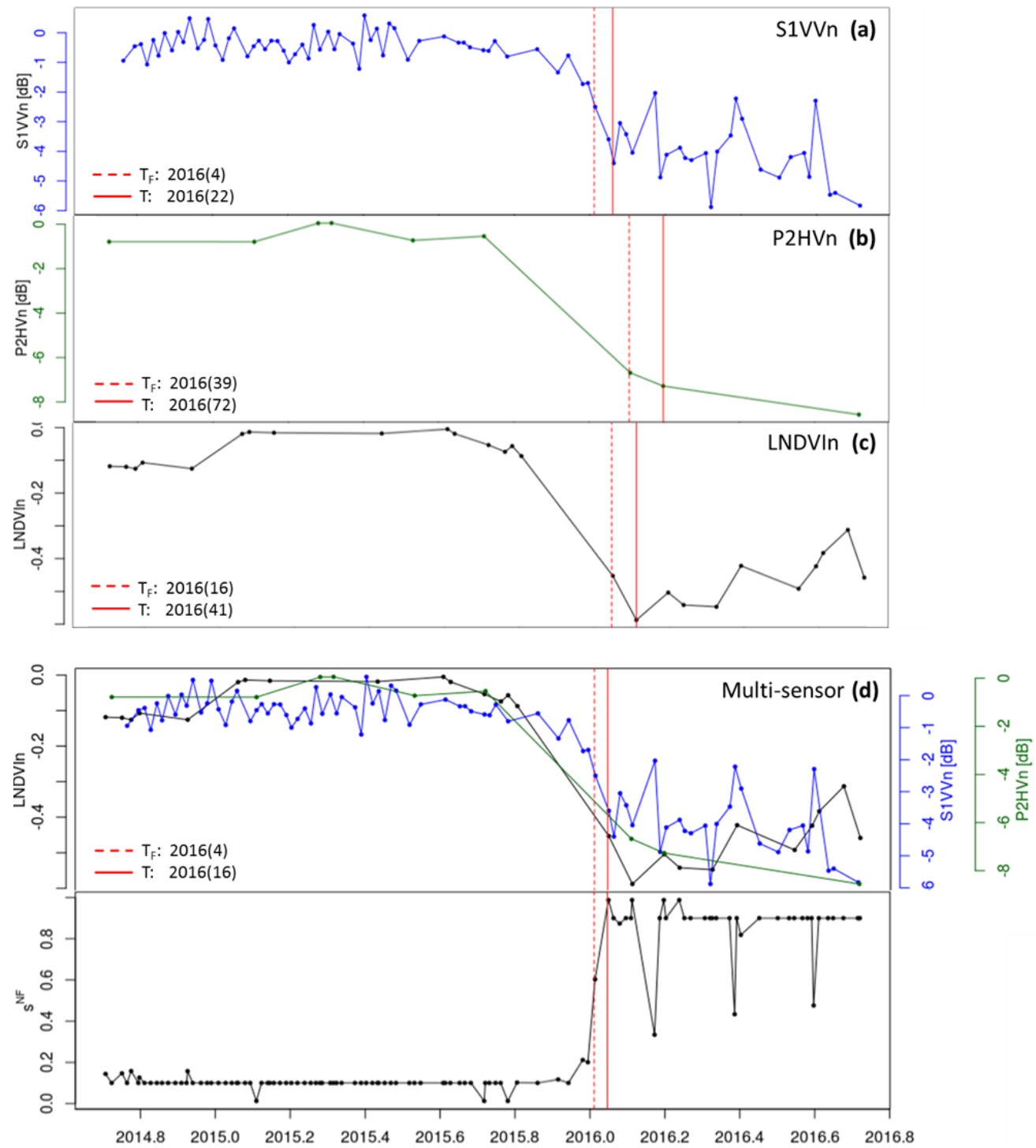

Figure 6.8. Pixel example showing detected deforestation, for the single-sensor cases, S1VVn (a), P2HVn (b), LNDVIn (c) and the multi-sensor case (d). For the multi-sensor case the time series of conditional NF probabilities, $\mathrm{s}^{\mathrm{NF}}$, is shown in addition (d-bottom). The time at which the deforestation was first flagged $\left(\mathrm{T}_{\mathrm{F}}\right)$ and the time at which it was confirmed $(\mathrm{T})$ are given as the day of year. The reference time at which the change was first visible in the satellite time series $\left(\mathrm{T}_{\text {ref }}\right)$ is $2015(354)$ (day of year). The adjusted reference time ( $\mathrm{T}_{\text {ref_adjusted }}$ ) is 2016(4). The time series are from the same pixel as Figure 6.7. 
(a) LNDVIn
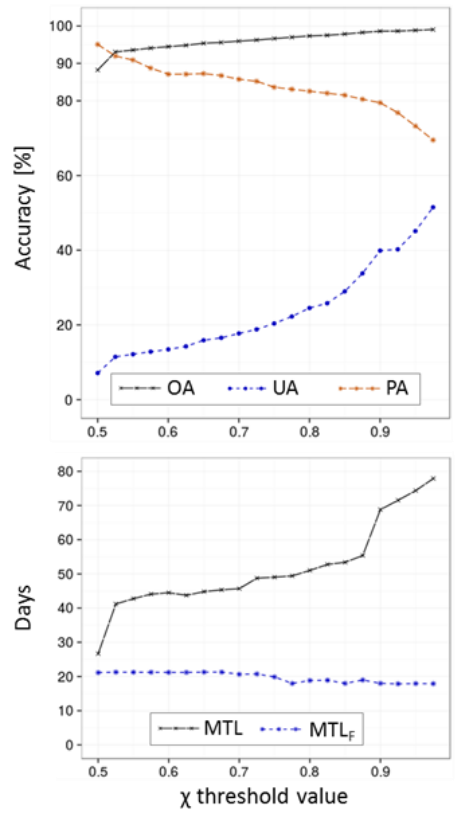

(b) S1VVn
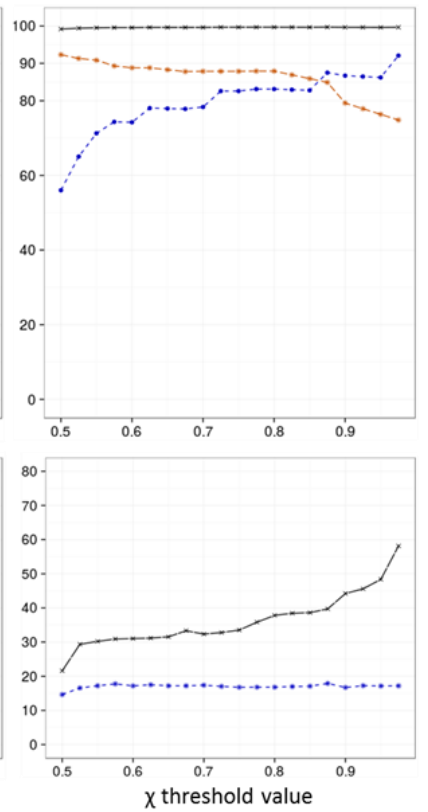

(c) Multi-sensor (LNDVIn, S1VVn, P2HVn)
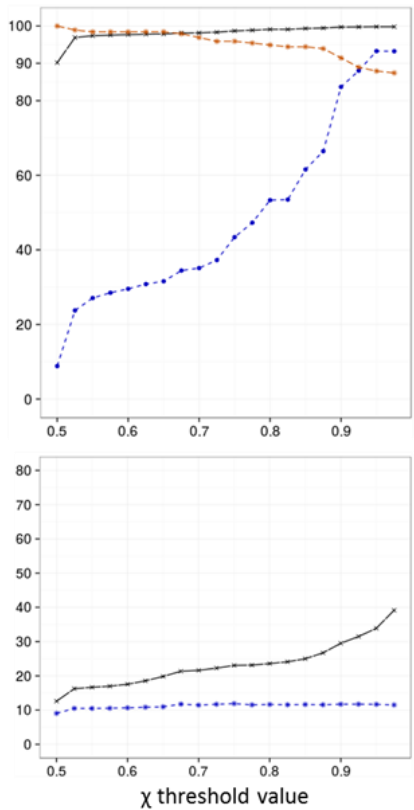

Figure 6.9. Spatial accuracy $(\mathrm{OA}=$ overall accuracy; $\mathrm{PA}$ and $\mathrm{UA}=$ producer's and user's accuracies of the deforestation class), and temporal accuracy (MTL = mean time lag of confirmed deforestation events; MTLF = mean time lag of the date at which confirmed deforestation events were first flagged) as a function of increasing $X$ threshold values, separately for S1VVn (A), LNDVIn (b) and the multi-sensor case (S1VVn, P2HVn, LNDVIn; c). 

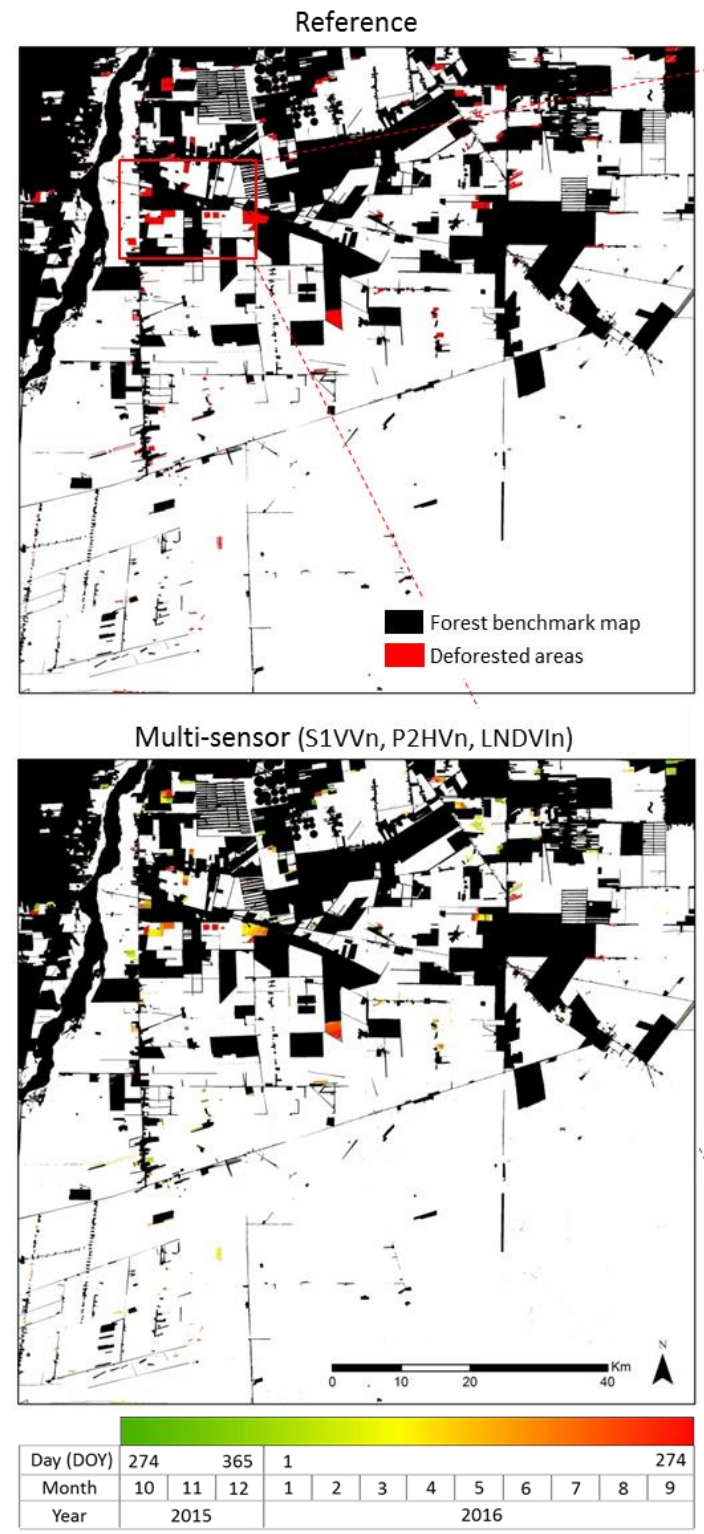
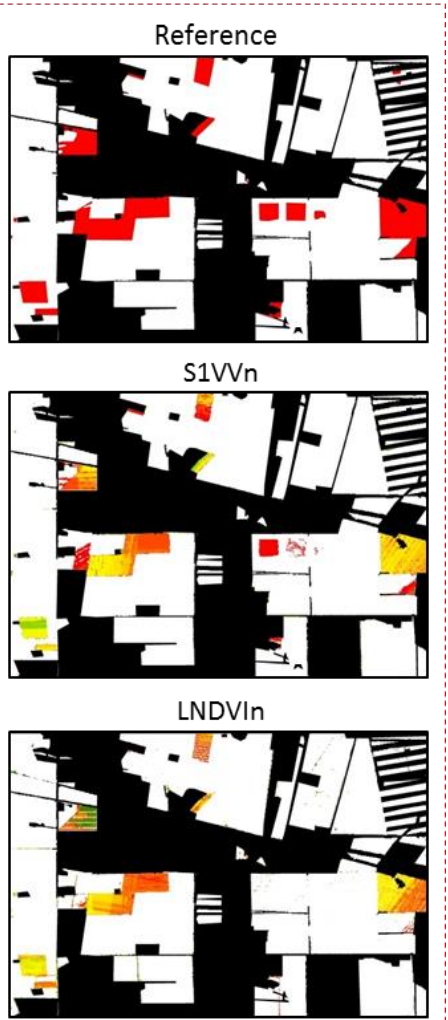

Multi-sensor

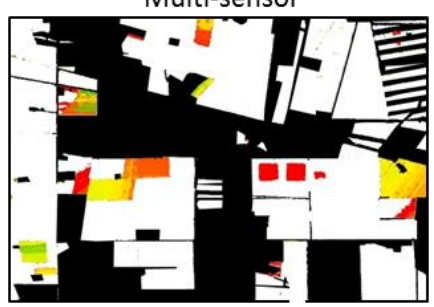

Figure 6.10. Deforestation events detected for multi-sensor case (S1VVn, P2HVn, LNDVIn) for the one year monitoring period (2015-10-01 - 2016-09-30) at the dry forest study site in Santa Cruz, Bolivia (bottom left). Reference forest benchmark map and deforested areas (top left) were digitized from the original images. For a subset (squared red box in reference map), single-sensor results for S1VVn and LNDVIn are compared with the multi-sensor results (right panel). 


\subsection{Discussion}

In this chapter we presented the first study on multi-senor SAR-optical NRT deforestation detection in a tropical dry forest, and we combined newly available dense Sentinel-1 time series with ALOS-2 PALSAR-2 and Landsat 7 and 8 data. We demonstrated that spatial normalisation can be used to reduce dry forest seasonality in the SAR time series in order to combine them with optical time series for NRT deforestation detection using a probabilistic approach. Our results for a dry tropical forest site in Bolivia show that deforestation events were detected more timely with Sentinel-1 than with Landsat or PALSAR-2. The spatial and temporal accuracies further improved beyond the single-sensor results when combining observations in a multisensor approach. We improved the precision of the reference data derived from the multi-sensor satellite time series, which enabled a more robust estimation of the temporal accuracy. When aiming for the lowest map area bias, the overall accuracy of our multi-sensor map was $99.8 \%$, with deforestation events detected with a user's accuracy of $88 \%$ and a producer's accuracy of $89 \%$. We estimated $71 \mathrm{~km}^{2}$ of newly deforested areas. This relatively high forest loss within one year can be attributed to large scale commercial logging activities which are typical for this area (Hamunyela, Verbesselt, Bruin, et al. 2016). Deforestation events were detected with a MTL of 31 days. This is more than one week earlier than when using single-sensor S1VVn results (MTL = 39 days), and even six weeks earlier than when using single-sensor LNDVIn results (MTL $=78$ days). The ability to detect deforestation events with such a high spatial and temporal accuracy highlights the advantage of combining medium spatial resolution time series data from multiple SAR and optical sensors.

\subsubsection{Optimized threshold selection for different use cases}

We found a clear trade-off between spatial and temporal accuracy when selecting the $X$ threshold value for deforestation monitoring. While the UA of the deforestation class improved for increasing $X$ threshold values, the PA of the deforestation class and temporal accuracy (MTL) decreased (Figure 6.9c). In other words, an increasing confidence to accurately detect deforestation events is associated with omitted and delayed detected changes. This trade-off confirms the findings of previous studies which evaluated the performance of satellite based time series change detection (Zhu et al. 2012; Hamunyela, Verbesselt, de Bruin, et al. 2016; Reiche, Verbesselt, et al. 2015). No single X threshold value satisfies the highest UA, PA and temporal accuracy. The selection of the $X$ threshold value is therefore dependent on the monitoring requirements of the user and is always associated with an error.

Here, we discuss four key use cases based on the multi-sensor results (Figure 6.9c). Table 6.1 provides an overview of the use cases and lists the $X$ 
threshold value used to generate the results together with the associated OA, UA and PA of the deforestation class, the estimated area bias and the MTL.

Table 6.1. Key use cases for detecting deforestation based on the multi-sensor results (Figure 6.9c). For each use case, the $\mathrm{X}$ threshold value used to generate the results is listed together with the associated overall accuracy (OA), user's and producer's accuracy of the deforestation class (UA \& PA), the estimated area bias and the mean time lag of detected deforestation (MTL).

\begin{tabular}{lcccccc}
\hline Use case & $\begin{array}{c}\mathrm{X} \text { threshold } \\
\text { value }\end{array}$ & $\begin{array}{c}\text { OA } \\
(\%)\end{array}$ & $\begin{array}{c}\text { UA } \\
(\%)\end{array}$ & $\begin{array}{c}\text { PA } \\
(\%)\end{array}$ & $\begin{array}{c}\text { Area bias } \\
\left(\mathrm{km}^{2}\right)\end{array}$ & $\begin{array}{c}\text { MTL } \\
(\text { days })\end{array}$ \\
\hline Fast alerting & 0.5 & 90.2 & 8.8 & 100 & 755 & 12 \\
Confident alerting & 0.8 & 99.1 & 53.3 & 94.9 & 50.4 & 22 \\
Accounting of changes & 0.925 & 99.8 & 88.0 & 88.9 & 0.4 & 31 \\
Target field surveys & 0.975 & 99.8 & 93.3 & 87.5 & -9 & 39 \\
\hline
\end{tabular}

The first use case (Fast alerting) describes the case in which a user is interested in detecting deforestation as quickly as possible. By choosing a low $\mathrm{X}$ threshold value of 0.5 , deforestation events are confirmed with a MTL of 13 days. This case basically relies on single observations to timely detect deforestation events, which are prone to noise, artefacts, and short-term image variability stemming from use multi-sensor data. In combination with the high sensitivity to flag change, this results in many false detections and large commission error $(\mathrm{UA}=8.8 \%)$. On the other hand no events were missed $(\mathrm{PA}=$ $100 \%$ ). These fast alerts might be used to identify potentially critical areas and/or for tasking high-resolution satellite data acquisitions to zoom in to specific hot spot areas.

The second use case (Confident alerting) describes a more realistic use case for NRT monitoring in which deforestation is detected based on more observations, less timely, but with an acceptable false alarm rate. By using a $X$ threshold value of 0.8 , deforestation events were detection with a MTL of 22 days, a commission error of $47 \%$ and an omission error of $5 \%$.

The third use case (Accounting of changes) reflects a user that aims for high confidence in area estimates (i.e. as used in greenhouse gas accounting; Defries et al. 2007) and wants to produce quality deforestation maps but does not need the highest temporal detail. Therefore, a low area bias is essential for accounting changes. The lowest area bias corresponded to the UA/PA cross-over point at $\mathrm{X}=0.925$ (area bias $=0.4 \mathrm{~km}^{2}$ ). A MTL of 31 days, basically allows for (on average) monthly accounting of forest loss area change.

The fourth use case (Target field surveys) presents the case in which a user wants to assess impacts of events on the ground with a dedicated field campaign. Deforestation events, therefore, need to be detected with the highest possible certainty to avoid field teams being sent to sites at which deforestation events were falsely detected. By using a $\mathrm{X}$ threshold value of 0.975 , a UA of 93.3\% was achieved indicating a low false alarm rate. Since many observations 
were used to increase the confidence to confirm deforestation, a high MTL of 39 days was the result and some changes were omitted.

In conclusion, there are trade-offs for multi-sensor satellite-based NRT deforestation monitoring depending on the intended use. A systems working in parallel optimizing monitoring towards these needs and addressing both alerting and area accounting could be followed. Increasing the use of local calibration data (i.e. from local experts, forest managers) can continuously and significantly improve the detection accuracy (Devries et al. 2016).

\subsubsection{Observation availability and implications for large area NRT monitoring}

Observation availability is considered the driving factor for the NRT performance of satellite based deforestation monitoring systems in the tropics (Hansen et al. 2016). With Sentinel-1, dense C-band SAR time series are now available globally. For the two year study period (2014-10-01 - 2016-09-30), a minimum of 57 Sentinel-1 observations ( $\sim 2.4$ obs. /month) were available for our study site (Figure 6.3a). The relatively flat topography of our study site, typical for the Amazonian basin, allowed the straightforward combination of ascending and descending images. In areas with strong relief, differing topographic effects in ascending and descending images should be considered; e.g. SAR layover and shadow at different locations. The two year study period corresponds to the first two mission years of Sentinel-1. For this period only one satellite (Sentinel-1A) was operating. It was used mainly to acquire data over prioritised areas and, therefore, many tropical areas were covered with few observations. With the launch of the second Sentinel satellite (Sentinel$1 \mathrm{~B})$, the new observation strategy of the Sentinel-1A/B constellation is in place (https://sentinel.esa.int/web/sentinel/missions/sentinel-1/observation-scenario).

With this new acquisition strategy, 12 day repeated acquisitions for all tropical regions are guaranteed; for many areas even in dual-polarisation mode (VV and VH). For many tropical regions ALOS-2 PALSAR-2 provides limited coverage per year. For the two year period, large parts of our study area were covered with four or less observations only. This did not allow a meaningful detection of deforestation using ALOS-2 PALSAR-2 data only. With the upcoming SAOCOM-1 (2017) and NISAR (2020) missions, more frequent and free of charge L-band SAR time series will become available.

While the acquisition strategy determines the observation availability of SAR sensors, cloud cover is the main limiting factor for optical sensors. For the two year period, cloud cover strongly reduced the number of valid per-pixel Landsat observations from 63 to a minimum of 13 observations ( 0.5 obs. /month) (Figure 6.3c). Our results confirm the findings of previous studies, which showed a large spatial variance of Landsat observation densities across space (Potapov et al. 2012; Sannier et al. 2014; Hansen et al. 2016). The issue 
of sparse optical time series over certain tropical regions, for example cloud forests, will remain even when combining multiple optical sensors, e.g. Landsat and Sentinel-2.

By combining observations from Sentinel-1, PALSAR-2 and Landsat, we were able to increase the number of available observations to a minimum of 65 observations ( $2.7 \mathrm{obs} . / \mathrm{month})$ and an average of 96 observations ( 4 obs./month) for the two year period (Figure 6.3d). We showed that providing regular observation throughout all seasons and locations results in increased spatial and temporal detection accuracies, which corroborates the findings of previous studies (Reiche, Verbesselt, et al. 2015; Reiche, de Bruin, et al. 2015).

\subsubsection{Spatial normalization to reduce dry forest seasonality}

The seasonal dry forest component was strongly pronounced in the optical LNDVI signal. The high sensitivity of the NDVI signal to change in photosynthetic activity that is associated with the change between wet and dry season, led to a drop of the LNDVI signal of up to 0.5 (Figure 6.7c1). LNDVI wet and dry season observations are reflected in a bimodal $\mathrm{F}$ distribution (Figure 6.6c). The dry season observations strongly overlapped with the NF class. This resulted in a weak $\mathrm{F} / \mathrm{NF}$ class separability ( $\mathrm{JM}=0.66$ ). Spatial normalisation reduced the seasonal dry forest component, as reflected in a unimodal $\mathrm{F}$ distribution, resulting in increased $\mathrm{F} / \mathrm{NF}$ separability $(\mathrm{JM}=1.01)$.

We found a weaker seasonal dry forest component for the S1VV and P2HV SAR backscatter signals. A weaker seasonal component was expected as SAR backscatter is not influenced by photosynthetic activity of the forest, but instead is sensitive to its structure and moisture (Ulaby et al. 1986). The backscatter signal increased from the dry to wet season in the range of $\sim 2 \mathrm{~dB}$. This is mainly explained by increased canopy moisture during the wet season (Woodhouse et al. 1999). Due to the relatively stable backscatter signal over forest, S1VV (JM = 1.14) and P2HV (JM = 1.91) already showed good F/NF separability prior to spatial normalisation. Nonetheless, spatial normalisation increased F/NF separability for S1VVn (JM = 1.44) and P2HVn $(\mathrm{JM}=1.98)$. The higher F/NF separability of P2HV can be mainly explained by two factors. First, the long wavelength L-band SAR penetrates the tropical forest canopy and backscattering is primarily caused by branches and trunks. After deforestation, the L-band backscatter strongly decreases causing a large contrast between forest and non-forest. Secondly, cross-polarised HV backscatter (P2HV) is more sensitive to forest change than single-polarised VV backscatter (S1VV) (Hoekman \& Quiriones 2000; Woodhouse 2005). The shorter wavelength of C-band SAR only partially penetrates the forest canopy, and primarily interacts with leaves and small branches. After forest removal, $\mathrm{C}$-band interacts in a similar way with the remaining understory vegetation and bushes. C-band also largely responds to soil surface roughness and 
moisture. Increased soil moisture after strong rain events for example, can lead to increased backscatter (Woodhouse et al. 1999). This effect is visible as temporary spikes in the S1VVn time series at NF conditions (Figure 6.7a). These effects lead to a generally lower F/FN separability when compared to Lband SAR (van der Sanden 1997; Ulaby et al. 1986; Woodhouse 2005). Utilizing the upcoming cross-polarised Sentinel-1 (VV and VH) time series promises increased $\mathrm{F} / \mathrm{NF}$ separability, as for example soil moisture differences will affect the signal less.

While spatial normalisation has been applied to reduce dry forest seasonality in Landsat NDVI data in earlier studies (Hamunyela, Verbesselt \& Herold 2016; Hamunyela, Verbesselt, Bruin, et al. 2016), its application on SAR data was first demonstrated in this study. We successfully applied spatial normalisation on both sparse (P2HV) and short SAR time series (P2HV and S1VV) acquired over dry forest. Our results confirm that spatial normalisation can be a valid and promising alternative approach to seasonal model fitting approaches (Hamunyela, Verbesselt \& Herold 2016) that rely on long and dense historical time series (Verbesselt, Hyndman, Newnham, et al. 2010; Zhu et al. 2012). We used a global percentile for spatial normalisation as our study site was covered homogeneously with dry forest. Computationally, a global percentile is more attractive, especially in an areas dominated by a single forest type. Where spatial normalisation is applied to an area with varying forest types and seasonality, a local window approach as demonstrated by Hamunyela, Verbesselt \& Herold (2016) should be used.

\subsubsection{Probabilistic approach for multi-sensor NRT deforestation detection}

Converting the original SAR (S1VVn and P2HVn) and optical (LNDVIn) information into NF probabilities allowed us to combine and analyse a single signal instead of separate SAR and optical signals. While we demonstrated the combination of three different sensors, the approach is flexible to combine observations from additional sensors, like e.g. Sentinel-2.

A major advantage of the proposed probabilistic approach is that it directly accounts for the different sensor specific F/NF class separability of S1VVn, P2HVn and LNDVIn observations, as the derived NF probabilities are used to calculate and update the conditional probability of deforestation. This allowed us to overcome some key limitations of the single-sensor time series. First, while $\mathrm{P} 2 \mathrm{HVn}$ showed the highest $\mathrm{F} / \mathrm{NF}$ class separability, NRT deforestation monitoring based on only $\mathrm{P} 2 \mathrm{HVn}$ observations was not reliable due to the very low data availability. For the multi-sensor case, however, the individual P2HVn observations were used. The high F/NF separability resulted in rather extreme NF probabilities of individual P2HVn observations, which in turn had a strong effect on the iterative Bayesian updating of the deforestation probability. Second, the lower F/NF class separability of LNDVIn and S1VVn 
often resulted in a reduced impact of single LNDVIn and S1VVn observation. However, this was compensated by the higher observation availability.

We demonstrated the combination of univariate SAR and optical time series. To make full use of the available reflectance spectra of optical sensors and different polarisations of SAR sensors, sensor specific F and NF class distributions should be derived from multi-variate instead of univariate feature spaces. Using VH-polarised Sentinel-1 observations in addition to the VVpolarised observations for example, can improve $\mathrm{F} / \mathrm{NF}$ class distributions (see Section 6.5.3).

In this study, we dealt with large area industrial logging activities which are typical in the region. For operationalisation and a wider area of application, the approach should be adapted to and tested for varying harvesting practices and more complex forest dynamics.

\subsubsection{Temporal accuracy and adjusted reference date}

We showed that the temporal accuracy is equally important as spatial accuracy when assessing the performance of satellite based NRT alert systems. Temporally dense ground information to assess deforestation events detected from dense SAR and optical time series is very rare. We showed that the reference date derived from the satellite time series itself, as commonly used in recent studies (Zhu \& Woodcock 2014a; Pratihast et al. 2015; Hansen et al. 2016; Reiche, Verbesselt, et al. 2015; DeVries et al. 2015), is associated with uncertainty about the true date for deforestation. The imprecision is related to the fact that the true date of deforestation occurred sometime between the date of the image at which the change is first visible (commonly considered as the reference date) and the date of the previous image in the time series. When combining the observations from Landsat, Sentinel-1 and ALOS-2 PALSAR-2 for our site, the imprecision of the derived reference date varied between 2 days and 39 days with an average of 13 days. By adjusting the reference date we were able to reduce the imprecision by fifty percent to an average of \pm 6 days. In this context, Sentinel-1 time series show great potential as regular and dense observations to enable the derivation of regular sampled reference data. This may allow a more comparable assessment of the temporal accuracy across different areas.

\subsection{Conclusion}

We demonstrated multi-sensor SAR-optical NRT deforestation detection in a tropical dry forest, and we combined time series observations from Sentinel-1, PALSAR-2 and Landsat 7 and 8. We successfully applied spatial normalisation to reduce the dry forest seasonality in the SAR and optical time series, and combined them in a probabilistic approach. Our results show that deforestation 
events were detected with a higher spatial and temporal accuracy when combining observations from multiple sensors than when using observations from a single sensor. We showed the unprecedented potential of dense Sentinel$1 \mathrm{C}$-band time series for providing dense observations and to compensate for sudden environmental effects. We improved the precision of reference data derived from the satellite time series itself and provided a more robust estimate of the temporal accuracy. We presented and discussed different use cases, and highlighted that deforestation detection is always associated with a trade-off between the user's, producer's and the temporal accuracy. Timely detection of deforestation events, for example, is commonly associated with a low user's accuracy and a high producer's accuracy. The operationalisation of the approach requires its adaption to deal with varying harvesting practices, more complex forest dynamics and to make use of all optical bands and SAR polarisations. In particular, the upcoming dense Sentinel-1 dual-polarised data promises to increase the capacity for NRT deforestation detection. In summary, combining multiple SAR and optical time series can guarantee regular and temporally dense observations at medium spatial resolution independent of weather, season and spatial location, and with this improves NRT deforestation monitoring in the tropics.

\section{Acknowledgements}

The work was funded by the European Commission Horizon 2020 BACI project (grant agreement 640176) and the European Space Agency (ESA) ForMoSa project - Forest Degradation Monitoring with Satellite Data Project (grant agreement 5160957022). It was also supported by the Global Forest Observation Initiative (GFOI) research and development programme. ALOS-2 PALSAR-2 data was provided by JAXA through the 6th Research Announcement for ALOS-2 (RA6) (PI No. 3036). 
Chapter

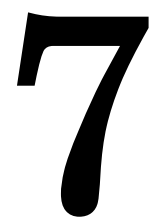

Synthesis 


\subsection{Main results}

Monitoring forest disturbances is crucial for initiatives that aim to protect of forest ecosystems from illegal exploitations, but detecting forest disturbances timely and accurately is challenging. The main objective of this thesis was to improve satellite-based forest change monitoring by tackling some key challenges that affect accurate and timely detection of forest disturbances. More specifically, the thesis assessed the extent to which combining contextual and temporal information in satellite image time series can help us to address the problems of: i) seasonality, ii) omission of small-scale disturbances, iii) inherent noise in satellite image time series and iv) inter-sensor differences in satellite image time series. To achieve the objective of the thesis, four research questions are formulated (Section 1.6), and each question is addressed through a core chapter, resulting into specific findings for each research question. Here, I summarise the main research findings for each question.

\subsubsection{How do we account for seasonality in irregular satellite image time series when aiming for accurate and timely detection of disturbances in dry forests (Chapter 2 and 6)?}

Accounting for seasonality is a pre-requisite for near real-time forest change detection using satellite data. Typically, existing change detection frameworks use a seasonal model to account for seasonality satellite image time series, but fitting a seasonal model is particularly challenging when the time series lacks sufficient observations or when the time series consist of observations acquired at irregular intervals. In this thesis, I tackled the problem of seasonality from a different perspective. Instead of using a seasonal model, I used contextual information in satellite images to reduce seasonality. More specifically, I developed a spatial normalisation approach in Chapter 2 which reduces seasonality, and applied that approach to Landsat image time series. I tested this spatial normalisation approach in both dry and humid tropical forests. The seasonality in the image time series for dry tropical forest was strong and poorly pronounced in the humid forest. Based on this analysis, the results showed that, in dry tropical forest, deforestation events were detected much earlier when using spatial normalisation approach to reduce seasonality than when using a seasonal model. In the dry tropical forest, the median temporal detection delay for deforestation from the spatial normalisation approach was two observations, which was seven times shorter than the median temporal detection delay from the seasonal model approach (15 observations). The difference in temporal detection delay between the spatial normalisation approach and the seasonal model approach in the humid tropical forest was not significant. This non-significant difference in temporal detection delay at the humid tropical forest site could be attributed to inherent low seasonality in the image time series over evergreen forests. Unlike strong 
seasonality, lowly pronounced seasonality in humid forest can hardly disguise the impact of deforestation on the time series.

I found that the differences in overall spatial accuracy between the spatial normalisation approach and seasonal model approach were also not significant in both dry and humid tropical forests. With a critical view, this nonsignificance in spatial accuracy is contradictory to the findings on temporal detection delay. Ironically, if the forest disturbances are detected early when using the spatial normalisation approach, then the spatial accuracy for the spatial normalisation approach should also be better than that of the seasonal model approach. A non-significant difference in spatial accuracy can be attributed to the way the validation approach was implemented. I sequentially tracked forest disturbances over a longer period, but the validation was based on single forest disturbance map which contains all changes detected over such long period. As result, even if the deforestation events were initially omitted, in case of the seasonal model approach, they could still be detected on a later stage. An appropriate monitoring and validation strategy would therefore been to bin the monitoring period into several monitoring periods, and generate a forest disturbance map for each period. The accuracy for each monitoring period could then be assessed separately. Such approach would be more robust and informative on the difference between spatial normalisation and seasonal model approach. Nonetheless, the results for this analysis show that using contextual information to reduce seasonality in satellite image time series is a robust alternative to a seasonal model approach, and can improve near realtime detection of forest disturbances, especially in dry forest.

In Chapter 2, I only demonstrated the spatial normalisation approach to optical image time series. Yet, near real-time forest change monitoring is increasingly done using a combination of optical and Synthetic Aperture Radar (SAR) image time series. Cognisant of this fact, in Chapter 6, this thesis further evaluated whether spatial normalisation approach can also be extended to SAR time series. It should be noted however, that SAR signal is not influenced by photosynthetic activity of the forest, but by forest structure and moisture (Ulaby et al. 1986). Yet, since the canopy moisture for forest especially for the dry forest, varies significantly between the wet and dry season because of the drying of the leaves, some form of seasonality is still expected in SAR time series. Therefore, in chapter 6, spatial normalisation approach was applied to Sentinel-1 and ALOS-2 PALSAR-2 time series to evaluate whether seasonal variations can be reduced by normalising SAR signal using contextual information. The results from this analysis show that spatial normalisation can also be used to reduce seasonal variations in SAR time series, thus increasing the separability between forest and non-forest observations. Forest change results from this analysis confirm that combining observations from multiple sensors improves the detection of forest disturbances. The results also confirm that timely detection of forest 
disturbances is typically accompanied by many false alerts, resulting in extremely low user's accuracy.

\subsubsection{What is the added-value of using both spatial and temporal information when monitoring forest disturbances from satellite image time series (Chapter 3 and 4)?}

In the past, monitoring forest disturbances using satellite data was based on the analysis of single pixel-time series, thus exploiting spectral and temporal information only. Contextual information is not included in deciding whether a pixel has been disturbed or not. In Chapter 3, I demonstrated how contextual and temporal information in satellite image time series can be combined and exploited to detect forest disturbances in near real-time. I proposed a data-driven space-time change detection method that forest disturbance as an extreme event within a space-time data cube of satellite image time series. Contextual and temporal information is combined by reducing the seasonal variations in image time series through spatial normalisation, and subsequently treat each deseasonalised observation in the local data cube as an independent observation. Forest disturbance is identified based on the distribution of deseasonalised observations in the data cube. I showed that forest disturbances can still be detected reliably even when the reference period only contains one-year of data. In this context, the proposed change detection approach provides an opportunity to detect forest disturbances using image time series with scarce historical observations. In addition, by leveraging both contextual and temporal information, there would be no need to pre-process huge amounts of historical data when monitoring forest disturbances. The proposed approach for combining contextual and temporal information in satellite data may however face challenges in mixed forests where deciduous and evergreen forests coexist within short distances mainly because of the way seasonality is reduced in the time series.

Producing forest disturbance maps that have both high spatial and temporal accuracy is one of the major challenges facing near real-time forest change monitoring. This thesis aimed at addressing this challenge by using a set of space-time features to reduce false detections when aiming for timely detection of forest disturbances. In Chapter 4, I demonstrated that achieving both high spatial and temporal accuracy is possible by using a set of space-time features extracted from local data cubes of satellite image time series to predict forest disturbance. Using a set of space-time features to confirm forest disturbance increases the capacity to reject many false detections, without compromising the temporal accuracy. I found space-time features related to change in spatio-temporal variability, and spatio-temporal association with non-forest areas, to be the main predictors for forest disturbance. The magnitude of change and two consecutive negative anomalies, which are widely 
used to distinguish forest disturbances from false detections, were not the main predictors for forest disturbance. However, it is should be noted that the importance of each space-time feature as predictor for forest disturbance may vary from one area to another, depending on the nature of forest disturbance. Nonetheless, like in Chapter 3, the findings in Chapter 4 emphase the importance of combining contextual and temporal information when aiming for accurate and timely detection forest disturbances. In particular, I showed that relying only on temporal information is not sufficient for accurate and timely detection of forest disturbances, especially when monitoring forest disturbances in areas where the disturbances occur at small spatial scale and in gradual manner.

\subsubsection{How can we combine observations from multiple satellites using space- time information to improve near real-time forest change monitoring (Chapter 5 and 6)?}

Accurate and timely detection of forest disturbances using satellite data can only be achieved when using observations from multiple satellites. However, earth observing satellites often have different radiometric calibrations, viewing angles, orbit times, and spatial and spectral resolutions. The unit of measurement also differ between SAR and optical. These intersensor differences complicate the combination of multi-sensor time series in two ways. First, the inter-sensor differences propagate disjoints in the time series which can amplify false detections. Second, deseasonalisation of the multi-time series cannot be done using a single seasonal model, especially when the sensors are using different measurement units.

In Chapter 5, I demonstrated that inter-sensor differences in optical-time series can be reduced through spatial normalisation. The differences are not entirely removed from the time series, but they are significantly reduced resulting into temporally consistent time series suitable for forest change detection. More specifically, using contextual information to harmonise Landsat and Sentinel-2 time series allowed for monitoring of forest disturbances at $10 \mathrm{~m}$ Sentinel-2 resolution while using Landsat time series as historical reference. Monitoring forest disturbance at $10 \mathrm{~m}$ Sentinel-2 resolution improved the detection of forest disturbances when compared to $30 \mathrm{~m}$ resolution.

In Chapter 6, the problem of seasonality in multi-sensor time series was tackled through spatial normalisation. More specifically, spatial normalisation was used to reduce seasonality in SAR and optical time series, thus facilitating the integration of multiple SAR and optical time series to monitor forest disturbances at dry tropical forest in near real-time. By using spatial normalisation, the use of sensor-specific seasonal model to account for seasonality was avoided, thus enabling for rapid combination of observations 
multiple satellites. In the Chapter 6, spatial normalisation approach was modified slightly. Instead of dividing each observation with $\mathrm{P}_{95}$, we subtracted $\mathrm{P}_{95}$ from each observation in order to preserve the magnitude of change. The inter-sensor differences between SAR and optical time series were instead reduced by converting observations into probability of forest (Reiche, de Bruin, et al. 2015). As result, in this Chapter, we could not conclude whether the spatial normalisation approach which is used in Chapter 5 to reduce intersensor differences in Landsat-Sentinel-2 time series can also be used to reduce inter-sensor differences in SAR-optical time series.

In Chapter 6, we showed that combining observations from Sentilel-1 and Landsat sensors leads to accurate and timely detection of forest disturbances, but timely detection of forest disturbances is accompanied by high commission error. In chapter 4 and 5, we showed that the commission error can be reduced by using a set of space-time features to confirm forest disturbances. Potentially space-time features can also reduce false detections in case of SAR-optical time series, but monitoring of forest disturbances from SAR-optical time series should shift from single pixel-analysis to space-time change analysis to take advantage of the space-time features.

\subsection{Reflection and outlook}

\subsubsection{Reducing inter-sensor differences in SAR-optical time series through spatial normalisation}

In chapter 5, I demonstrated that inter-sensor differences in multi-optical sensor time series can be reduced through spatial normalisation to generate temporally consistent multi-sensor time series suitable for monitoring forest disturbances. I did not however investigate whether spatial normalisation can also be used to reduce inter-sensor differences between SAR and optical time. So, one of the outstanding questions that this thesis did not answer is whether spatial normalisation can be an alternative approach for reducing inter-sensor differences between SAR and optical time series. Preliminary results show that spatial normalisation can also reduce inter-sensor differences in SARoptical time series, resulting in temporally consistent multi-sensor time series (Figure 7.1), suitable for forest change detection (Figure 7.2). Inter-sensor differences in SAR-optical time series are reduced by first inverting each SAR value in each image (E.q. 7.1), before applying spatial normalisation as proposed in Chapter 2.

$$
\rho=\frac{1}{|q|}
$$


where $|q|$ is the absolute value of the original SAR backscatter, and $\rho$ is an inverted SAR backscatter. Future work should aim at assessing whether SARoptical time series integrated through spatial normalisation can also result in accurate and timely detection of forest disturbances. Reducing inter-sensor differences between SAR and optical time series through spatial normalisation is advantageous because: i) the user can apply any change detection approach of choice, including curve-fitting approaches e.g. (Zhu et al. 2012; Verbesselt et al. 2012; Kennedy et al. 2010).

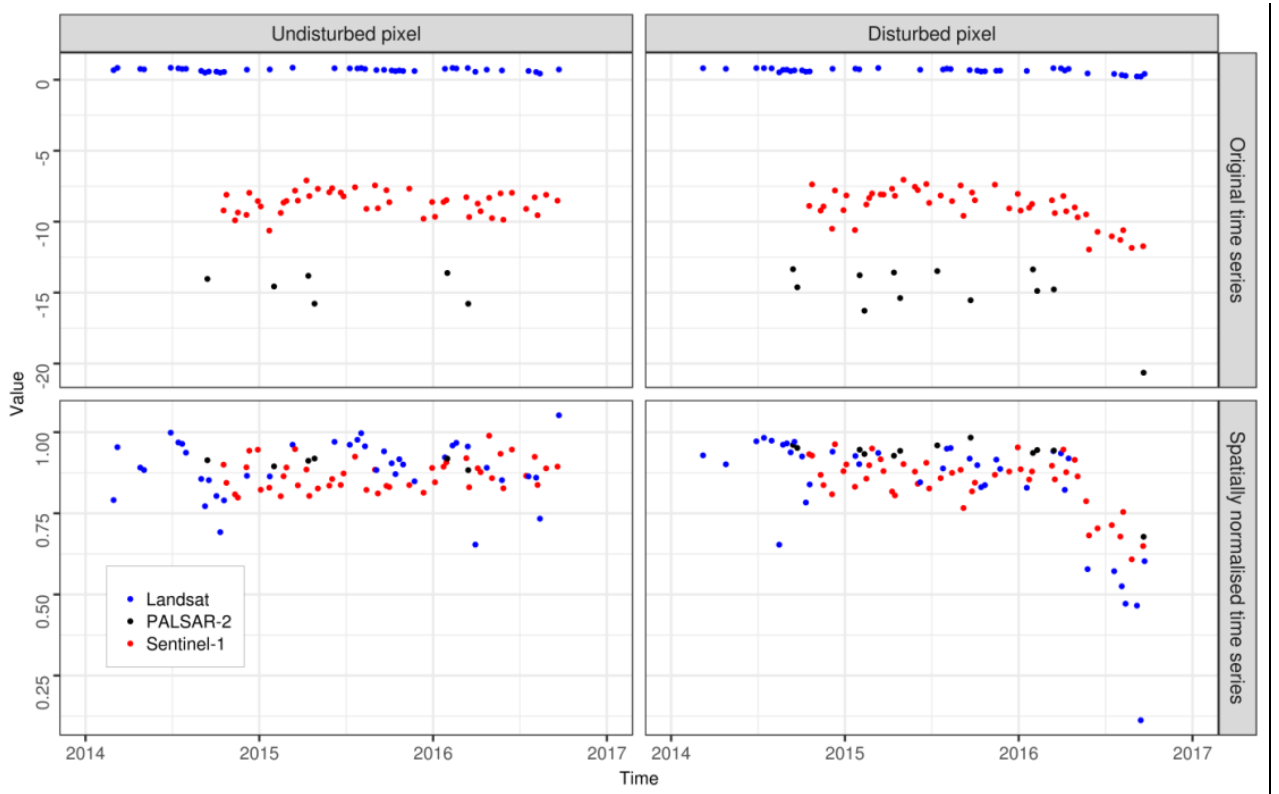

Figure 7.1: An example of original and spatially normalised pixel-time series for Landsat NDVI, Sentinel-1 and ALOS-2 PALSAR-2 backscatter at undisturbed and disturbed pixels from a dry tropical forest in Bolivia. Note that the variations in the original time series, especially for Landsat, are not evident because of the scale on y-axis. 


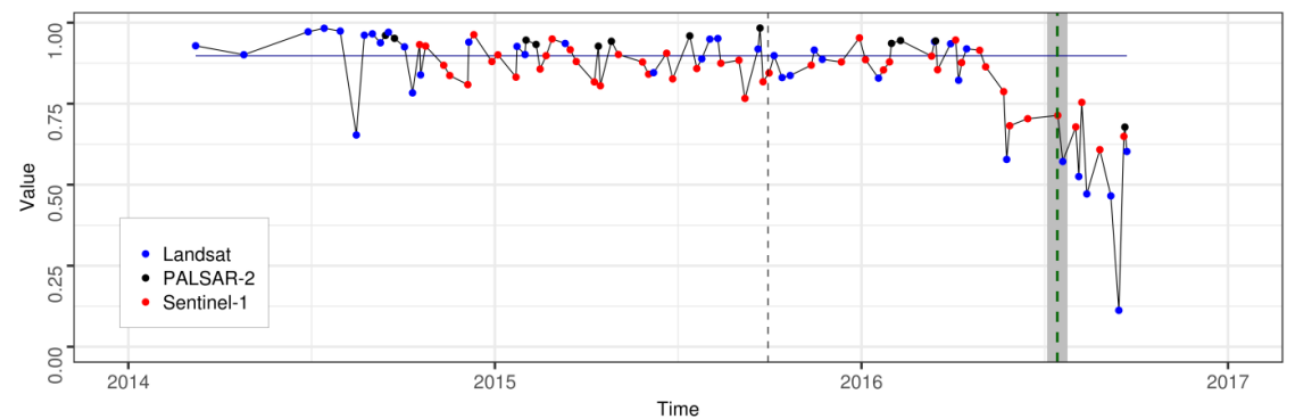

Figure 7.2: An example of forest disturbance detected from spatially normalised multi-sensor time series for Landsat, Sentinel-1 and ALOS-2 PALSAR-2 using the BFAST (Verbesselt et al. 2012). The time series is for the disturbed pixel shown in Figure 7.1. The solid horizontal line (dark blue) is the fitted mean model, whereas the dashed vertical lines indicate the start of the monitoring period (black) and the date in which a forest disturbance was detected (dark green). Here, the forest disturbance was detected in Sentinel-1 image.

\subsubsection{Space-time forest change monitoring using SAR-Optical time series}

This thesis demonstrated that space-time features, extracted from data cubes of satellite image time series, improve the detection of forest disturbances at sub-annual scale by increasing the capacity to reject false detections without compromising the temporal accuracy. However, in this thesis, I used the optical image time series to demonstrate the idea of using space-time features to confirm forest disturbances. Yet, forest change monitoring is increasingly done using a combination of SAR and optical time series. With this fact in mind, it is important that the idea of using space-time features is also extended to a combined SAR-optical time series. To do this, however, a space-time change detection framework that can integrate SAR and optical time series is needed. There is high potential that the space-time change approach I developed in Chapter 3 and 4 can accommodate SAR- optical time series. This is so because, like seasonal variations, inter-sensor differences in SAR-optical time series can also be reduced through spatial normalisation (Figure 7.1 and 7.3). Figure 7.3 shows the distribution of SAR and optical observations in local data cube before and after spatial normalisation. Potentially, with this distribution after spatial normalisation, forest disturbance can be identified as extreme event in SAR- optical data cube, and space-time features can also be extracted from the data cube. However, future work should aim at investigating whether detecting forest disturbances from SAR-optical time series using the space-time change approach also leads to accurate and timely detection of forest disturbances. 

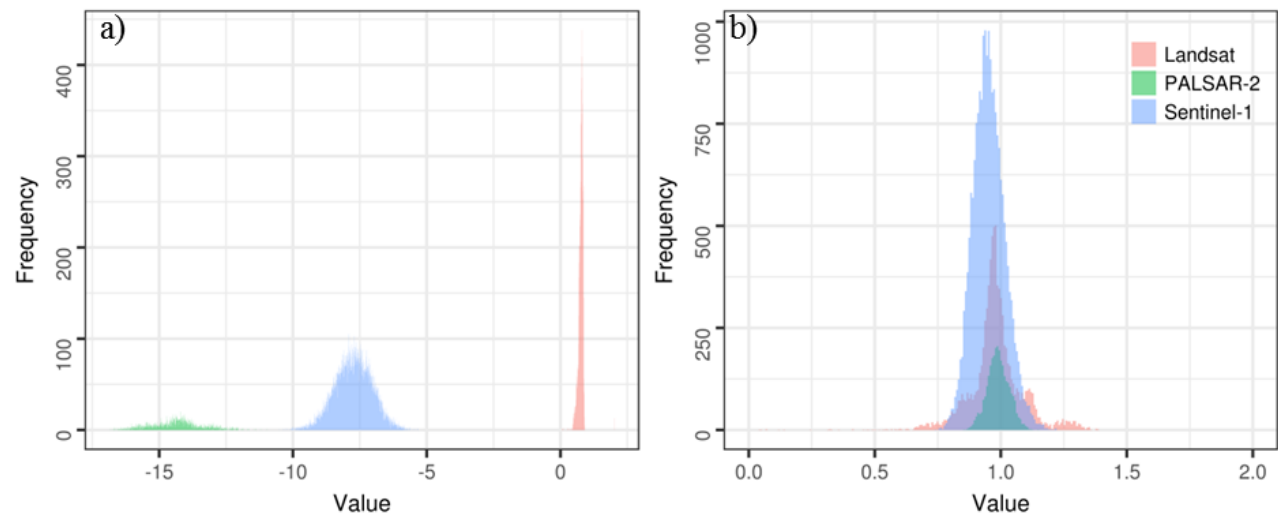

Figure 7.3: Distribution of Landsat NDVI, and Sentinel-1 and ALOS-2 PALSAR-2 backscatter in a local cube (a) before and (b) after spatial normalisation

\subsubsection{Toward timely characterisation of the drivers of forest change}

Apart from forest change itself, timely and accurate wall-to-wall information on the type and drivers of forest change is critically needed to understand why the forest cover is disturbed (Hansen et al. 2010). Currently, wall-to-wall information on the drivers for forest change is approximated from satellite data retrospectively (De Sy et al. 2015). With advancement in forest change detection realised in this thesis, whereby both spatial and temporal information are exploited for forest change detection, coupled recent advancement in machine learning and computer vision (Huang et al. 2006; Lary et al. 2016; Akram et al. 2017), there is now a unique opportunity to investigate whether drivers of forest change can be predicted from space-time features extracted from satellite image time series. Recently, features extracted from satellite images were used to predict spatially-explicit poverty levels using machine learning (Jean et al. 2016). There is also a need to investigate whether existing approaches for mapping forest regrowth (Devries, Decuyper, et al. 2015) can be improved by exploiting space-time information in satellite data.

Characterisation of forest changes should ideally determine the type of forest change (deforestation, forest degradation, or forest regrowth) and the driver of forest change (e.g. agriculture or timber harvesting), as well as the agent causing the forest disturbances (human or natural). Characterising drivers of forest change in this way would lead to timely and wall-to-wall determination of types and the drivers of forest change, thus contributing significantly towards the measurement, reporting and verification (MRV) systems under REDD+ mechanism (Reduce emissions from deforestation and forest degradation). To achieve this, however, elaborate training and validation data sets are essential. Acquiring elaborate training and validation data sets 
for forest change is challenging (Cohen et al. 2010), but, with recent advancement in citizen science and community-based forest monitoring (Pratihast et al. 2012; Pratihast et al. 2016; Devries et al. 2016; Pratihast et al. 2014), elaborate training and validation data sets for drivers of forest change can be acquired. Training and validation data sets acquired through citizen science and community-based forest monitoring can be complemented with data sourced through daily high-resolution image from nanosatellites sensors (e.g. Planet's satellites) to also acquire training and validation data in areas where local people could not access.

\subsubsection{Optimising space-time forest change detection for large-area monitoring}

The space-time change detection framework proposed in this thesis was tested at three test sites, namely in Bolivia, Brazil and Ethiopia. These sites have major differences. For example, the Bolivian site is a dry tropical forest that exhibits strong seasonality. Forest disturbances at this site are mainly large blocks of deforestation events caused by industrial agricultural expansion. The forest at the Brazilian site is an evergreen tropical forest, and the forest disturbances are heterogeneous, composed of large and small-scale disturbances. The Ethiopian site is a moist Afromontane broadleaf evergreen forest with moderate seasonality. At this site, forest disturbances are occurring mainly at small spatial scale, and are caused by small-holder agriculture, human settlements expansion, industrial coffee plantations, and domestic firewood and charcoal extractions. Although these three sites represent a combination of some major differences in forest types and disturbances, they do not represent all important types of forest disturbance regimes that occur at regional or global scale. Therefore it is not possible to conclude that the proposed space-time change detection framework is ready for upscaling to regional or global scale. The space-time change detection framework should be evaluated further especially in dry tropical forest areas where disturbances occur at small spatial scales and in gradual manner, to properly optimise the approach for large-area forest change monitoring. In conclusion, it is critical that forest change detection methods are sufficiently flexible to adapt to different regions with varying forest disturbances in order to improve largearea forest monitoring.

\subsubsection{Monitoring forest changes in a big earth observation data era: challenges and opportunities}

With recent advent of Copernicus Sentinel satellites, such as Sentinel-1 and -2 sensors, coupled with existing Landsat sensors, free and open access medium resolution earth observation data with high temporal frequency are 
increasingly becoming available. Such "big" earth observation data will have profound influence on how forest changes are monitored, especially on the monitoring frequency and the nature of disturbances that can be detected. For example, since it will be possible to have medium spatial resolution satellite image every few days even in cloudy tropical regions, mainly because of Sentinel-1 that penetrates clouds, forest disturbances especially small-scale disturbances caused for example by selective logging can be detected with high temporal details. However, monitoring forest changes using such voluminous earth observation data will present major challenges. For example, downloading and pre-processing such voluminous data and use it to monitor forest disturbances at high temporal frequency and over large area will be computationally challenging.

Computationally, space-time forest change monitoring is more expensive than single pixel-time series change monitoring. Currently, the space-time change detection approach presented in this thesis is implemented using $\mathrm{R}$ programming language, which is computationally not fast. This makes the approach relatively slow for upscaling to large areas. So, to upscale space-time forest change monitoring approach to large areas requires further research on how to computationally optimise it, especially in the context of big earth observation data. Recent developments in spatio-temporal detection of forest disturbances in multi-dimensional satellite data arrays (Lu et al. 2016) coupled with ongoing initiatives which aim to scale-up spatio-temporal analytics of earth observation using $\mathrm{R}$ and SciDB (http://r-spatial.org/r/2016/05/11/scalableearth-observation-analytics.html) have potential to accelerate research on space-time forest change monitoring using multi-sensor image time series. Furthermore, cloud-computing infrastructure i.e. Google Earth Engine, Earth Observation Data Centre for Water Resources Monitoring, NASA Earth Exchange and the planned European Open Science Cloud will be crucial for overcoming the challenges presented by large-area and frequency monitoring of forest changes using big earth observation data. However, for such cloudcomputing infrastructures to be more effective, the image pre-processing and change detection algorithms should remain open-source to enable reproducible science and to accelerate forest change monitoring. Overall, accurate, timely and consistent co-registration of data from multiple satellites is a key challenge that should be addressed to ensure exploitation of such multi-sensor data for forest change monitoring. 



\section{References}

Achard, F. et al., 2014. Determination of tropical deforestation rates and related carbon losses from 1990 to 2010. Global change biology, 20, pp.2540-2554.

Achard, F. et al., 2007. Pan-tropical monitoring of deforestation. Environmental Research Letters, 2(45022), p.11.

Aerts, R., Berecha, G. \& Honnay, O., 2015. Protecting coffee from intensification. Science, 347(6218), p.139.

Akram, T. et al., 2017. Towards real-time crops surveillance for disease classification: exploiting parallelism in computer vision. Computers and Electrical Engineering, 59, pp.15-26.

Alves, D.S., 2002. Space-time dynamics of deforestation in Brazilian Amazônia. International Journal of Remote Sensing, 23(14), pp.2903-2908.

Anderson, L.O. et al., 2005. Assessment of deforestation in near real time over the Brazilian Amazon using multitemporal fraction images derived from Terra MODIS. IEEE Geoscience and Remote Sensing Letters, 2(3), pp.315-318.

Arneth, A. et al., 2017. Historical carbon dioxide emissions caused by land-use changes are possibly larger than assumed. Nature Geoscience, 10, pp.7984 .

Asner, G.P., 2001. Cloud cover in Landsat observations of the Brazilian Amazon. International Journal of Remote Sensing, 22(18), pp.3855-3862.

Asner, G.P. et al., 2013. Elevated rates of gold mining in the Amazon revealed through high-resolution monitoring. PNAS, 110(46), pp.18454-18459.

Assunção, J., Gandour, C. \& Rocha, R., 2013. DETERring Deforestation in the Brazilian Amazon: Environmental Monitoring and Law Enforcement, Rio De Janeiro: Climate Policy Initiative.

Bonan, G.B., 2008. Forests and climate change: climate benefits of forests. Science, 320, pp.1444-1450.

Breiman, L.E.O., 2001. Random Forests. Machine Learning, 45, pp.5-32.

Carter, S. et al., 2015. Mitigation of agriculture emissions in the tropics: comparing forest land-sparing options at the national level. Biogeosciences Discussions, 12(7), pp.5435-5475. 
Chu, C., Stinchcombe, M. \& White, H., 1996. Monitoring structural change. Econometrica, 64(5), pp.1045-1065.

Ciais, P. et al., 2005. Europe-wide reduction in primary productivity caused by the heat and drought in 2003. Nature, 437(7058), pp.529-33.

Cleveland, R.B. et al., 1990. STL: A seasonal-trend decomposition procedure based on loess. Journal of Official Statistics, 6, pp.3-73.

Cohen, J.E., 2003. Human Population: The Next Half Century. Science, 302, pp.1172-1175.

Cohen, W.B. et al., 2017. How similar are forest disturbance maps derived from different Landsat time series algorithms? Forests, 8, p.98.

Cohen, W.B., Yang, Z. \& Kennedy, R., 2010. Detecting trends in forest disturbance and recovery using yearly Landsat time series: 2 . TimeSync - Tools for calibration and validation. Remote Sensing of Environment, 114(12), pp.2911-2924.

Coppin, P. et al., 2014. Digital change detection methods in ecosystem monitoring: a review. International Journal of Remote Sensing, 25(9), pp.1565-1596.

Coumou, D. \& Rahmstorf, S., 2012. A decade of weather extremes. Nature Climate Change, 2(7), pp.1-6.

Davis, R. a. \& Mikosch, T., 2008. Extreme value theory for space-time processes with heavy-tailed distributions. Stochastic Processes and their Applications, 118, pp.560-584.

DeFries, R. et al., 2007. Earth observations for estimating greenhouse gas emissions from deforestation in developing countries. Environmental Science \& Policy, 10(4), pp.385-394.

Defries, R.S., Hansen, M.C. \& Townshend, J.R.G., 2000. Global continuous elds of vegetation characteristics: a linear mixture model applied to multi-year $8 \mathrm{~km}$ AVHRR data. International Journal of Remote Sensing, 21(6), pp.1389-1414.

Devries, B. et al., 2016. Characterizing forest change using community-based monitoring data and Landsat time series. PLoS ONE, 11(3), p.e0147121.

Devries, B., Verbesselt, J., et al., 2015. Robust monitoring of small-scale forest disturbances in a tropical montane forest using Landsat time series. Remote Sensing of Environment, 161, pp.107-121.

Devries, B., Decuyper, M., et al., 2015. Tracking disturbance-regrowth dynamics in tropical forests using structural change detection and Landsat time series. Remote Sensing of Environment, 169, pp.320-334. 
Diniz, C.G. et al., 2015. DETER-B: The New Amazon Near Real-Time Deforestation Detection System. IEEE Journal of Selected Topics in Applied Earth Observations and Remote Sensing, 8(7), pp.3619-3628.

Dresen, E. et al., 2014. Fuelwood savings and carbon emission reductions by the use of improved cooking stoves in an afromontane forest, Ethiopia. Land, 3, pp.1137-1157.

Dutrieux, L.P. et al., 2015. Monitoring forest cover loss using multiple data streams, a case study of a tropical dry forest in Bolivia. ISPRS Journal of Photogrammetry and Remote Sensing, 107, pp.112-125.

Fan, X. \& Liu, Y., 2017. A Generalized Model for Intersensor NDVI Calibration and Its Comparison With Regression Approaches. IEEE Transactions on Geoscience and Remote Sensing, 55(3), pp.1842-1852.

FAO, 2015. Global Forest Resources Assessment 2015,

Fearnside, P.M., 1996. Amazonian deforestation and global warming: carbon stocks in vegetation replacing Brazil's Amazon forest. Forest Ecology and Management, 80, pp.21-34.

Fearnside, P.M., 2000. Global warming and tropical land-use change: greenhouse gas emissions from biomass burning, decomposition and soils in forest conversion, shifting cultivation and secondary vegetation. Climatic Change, 46, pp.115-158.

Fisher, B., 2010. African exception to drivers of deforestation. Nature Geoscience, 3, pp.375-376.

Foody, G., 2002. Status of land cover classification accuracy assessment. Remote Sensing of Environment, 80(1), pp.185-201.

GFOI, 2015. GFOI R \& D Plan for 2015+: An action plan for advancing priority R\&D topics related to the use of Remote Sensing in National Forest Monitoring. Version 1.1, (Version 1.1), p.105.

Glasbey, C.A., Graham, R. \& Hunter, A.G.M., 2001. Spatio-temporal variability of solar energy across a region: a statistical modelling approach. Solar Energy, 70(4), pp.373-381.

Green, R.E. et al., 2005. Farming and the Fate of Wild Nature. Science, 307, pp.550-556.

Griffiths, P. et al., 2012. Using annual time-series of Landsat images to assess the effects of forest restitution in post-socialist Romania. Remote Sensing of Environment, 118, pp.199-214.

Grogan, K. et al., 2015. Cross-border forest disturbance and the role of natural rubber in mainland Southeast Asia using annual Landsat time series. 
Remote Sensing of Environment, 169, pp.438-453.

Hammer, D., Kraft, R. \& Wheeler, D., 2014. Alerts of forest disturbance from MODIS imagery. International Journal of Applied Earth Observation and Geoinformation, 33, pp.1-9.

Hammer, D., Kraft, R. \& Wheeler, D., 2009. FORMA: Forest Monitoring for Action-Rapid Identification of Pan-Tropical Deforestation Using Moderate-Resolution Remotely Sensed Data, Rochester, New York: Centre for Global Development.

Hamunyela, E., Verbesselt, J., Bruin, S. De, et al., 2016. Monitoring deforestation at sub-annual scales as extreme events in Landsat data cubes. Remote sensing, 8, p.651.

Hamunyela, E. et al., 2013. Trends in Spring Phenology of Western European Deciduous Forests. Remote Sensing, 5(12), pp.6159-6179.

Hamunyela, E. et al., Using space-time features to improve detection of forest disturbances using Landsat time series. Remote sensing, In review.

Hamunyela, E., Verbesselt, J. \& Herold, M., 2016. Using spatial context to improve early detection of deforestation from Landsat time series. Remote Sensing of Environment, 172, pp.126-138.

Hansen, M.. et al., 2016. Humid tropical forest disturbance alerts using Landsat data. Environmental Research Letters, 11(3), p.34008.

Hansen, M.C. et al., 2000. Global land cover classification at $1 \mathrm{~km}$ spatial resolution using a classification tree approach. International Journal of Remote Sensing, 21(6), pp.1331-1364.

Hansen, M.C. et al., 2003. Global Percent Tree Cover at a Spatial Resolution of 500 Meters: First Results of the MODIS Vegetation Continuous Fields Algorithm. Earth Interactions, 7(10), pp.1-13.

Hansen, M.C. et al., 2013. High-resolution global maps of 21st-century forest cover change. Science, 342, pp.850-853.

Hansen, M.C. \& Defries, R.S., 2004. Detecting Long-term Global Forest Change Using Continuous Fields of Tree-Cover Maps from 8-km Advanced Very High Resolution Radiometer ( AVHRR ) Data for the Years 1982 - 99. Ecosystems, 7, pp.695-716.

Hansen, M.C. \& Loveland, T.R., 2012. A review of large area monitoring of land cover change using Landsat data. Remote Sensing of Environment, 122, pp.66-74.

Hansen, M.C., Stehman, S. V \& Potapov, P. V, 2010. Quantification of global gross forest cover loss. PNAS, 107(19), pp.8650-8655. 
Heimann, M. \& Reichstein, M., 2008. Terrestrial ecosystem carbon dynamics and climate feedbacks. Nature, 451, pp.289-292.

Hilker, T., Wulder, M.A., Coops, N.C., Linke, J., et al., 2009. A new data fusion model for high spatial- and temporal-resolution mapping of forest disturbance based on Landsat and MODIS. Remote Sensing of Environment, 113(8), pp.1613-1627.

Hilker, T., Wulder, M.A., Coops, N.C., Seitz, N., et al., 2009. Generation of dense time series synthetic Landsat data through data blending with MODIS using a spatial and temporal adaptive reflectance fusion model. Remote Sensing of Environment, 113(9), pp.1988-1999.

Hoekman, D.H. \& Quiriones, M.J., 2000. Land cover type and biomass classification using AirSAR data for evaluation of monitoring scenarios in the Colombian Amazon. IEEE Transactions on Geoscience and Remote Sensing, 38(2), pp.685-696.

Huang, C. et al., 2009. Dynamics of national forests assessed using the Landsat record: Case studies in eastern United States. Remote Sensing of Environment, 113(7), pp.1430-1442.

Huang, G., Zhu, Q. \& Siew, C., 2006. Extreme learning machine: Theory and applications. Neurocomputing, 70, pp.489-501.

Ichoku, C. et al., 2002. A spatio-temporal approach for global validation and analysis of MODIS aerosol products. Geophysical Research Letters, 29(12), p.1616.

Jean, N. et al., 2016. Combining satellite imagery and machine learning to predict poverty. Science, 353(6301), pp.790-794.

Jenkins, M., 2007. Prospects for Biodiversity. Science, 302, pp.1175-1177.

Joshi, N. et al., 2016. A Review of the Application of Optical and Radar Remote Sensing Data Fusion to Land Use Mapping and Monitoring. Remote Sensing, 8, p.70.

Ke, Y. et al., 2015. Characteristics of Landsat 8 OLI-derived NDVI by comparison with multiple satellite sensors and in-situ observations. Remote Sensing of Environment, 164, pp.298-313.

Kennedy, R.E., Yang, Z. \& Cohen, W.B., 2010. Detecting trends in forest disturbance and recovery using yearly Landsat time series: 1. LandTrendr - Temporal segmentation algorithms. Remote Sensing of Environment, 114(12), pp.2897-2910.

Laliberte, A.S., Browning, D.M. \& Rango, A., 2012. A comparison of three feature selection methods for object-based classification of sub-decimeter 
resolution UltraCam-L imagery. International Journal of Applied Earth Observation and Geoinformation, 15, pp.70-78.

Lamb, A. et al., 2016. The potential for land sparing to o set greenhouse gas emissions from agriculture. Nature Climate Change, 6, pp.488-492.

Lary, D.J. et al., 2016. Machine learning in geosciences and remote sensing. Geoscience Frontiers, 7(1), pp.3-10.

Lehmann, E.A. et al., 2012. Joint processing of Landsat and ALOS-PALSAR data for forest mapping and monitoring. IEEE Transactions on Geoscience and Remote Sensing, 50(1), pp.55-67.

Lehmann, E.A. et al., 2015. SAR and optical remote sensing: Assessment of complementarity and interoperability in the context of a large-scale operational forest monitoring system. Remote Sensing of Environment, 156, pp.335-348.

Leisch, F., Hornik, K. \& Kuan, C.-M., 2000. Monitoring structural changes with the generalized fluctuation test. Econometric Theory, 16, pp.835-854.

Lhermitte, S. et al., 2011. A comparison of time series similarity measures for classification and change detection of ecosystem dynamics. Remote Sensing of Environment, 115(12), pp.3129-3152.

Lhermitte, S. et al., 2010. A pixel based regeneration index using time series similarity and spatial context. Photogrammetric Engineering and Remote Sensing, 76(6), pp.673-682.

Li, P., Jiang, L. \& Feng, Z., 2014. Cross-Comparison of Vegetation Indices Derived from Landsat-7 Enhanced Thematic Mapper Plus (ETM+) and Landsat-8 Operational Land Imager (OLI) Sensors. Remote Sensing, 6, pp.310-329.

Lobell, D.B., Sibley, A. \& Ortiz-monasterio, J.I., 2012. Extreme heat effects on wheat senescence in India. Nature Climate Change, 2(3), pp.186-189.

Lu, M. et al., 2016. Spatio-temporal change detection from multidimensional arrays: Detecting deforestation from MODIS time series. ISPRS Journal of Photogrammetry and Remote Sensing, 117, pp.227-236.

Luyssaert, S. et al., 2008. Old-growth forests as global carbon sinks. Nature, 455, pp. $213-215$.

Lynch, J. et al., 2013. Choose satellites to monitor deforestation. Nature, 496(7445), pp.293-294.

Mandanici, E. \& Bitelli, G., 2016. Preliminary Comparison of Sentinel-2 and Landsat 8 Imagery for a Combined Use. Remote Sensing, 8, p.1014. 
Masek, J.G. et al., 2006. A Landsat surface reflectance dataset for North America, 1990-2000. IEEE Geoscience and Remote sensing letters, 3(1), pp.68-72.

Meehl, G.A. \& Tebaldi, C., 2004. More intense, more frequent , and longer lasting heat waves in the 21st century. Science, 305, pp.994-997.

Motohka, T. et al., 2014. Using time series PALSAR gamma nought mosaics for automatic detection of tropical deforestation: A test study in Riau, Indonesia. Remote Sensing of Environment, 155, pp.79-88.

Nepstad, D. et al., 2014. Slowing Amazon deforestation through public policy and interventions in beef and soy supply chains. Science, 344(6188), pp.1118-23.

Nepstad, D. et al., 2008. The end of deforestation in the Brazilian Amazon. Science, 326, pp.1350-1351.

Newbold, T. et al., 2015. Global effects of land use on local terrestrial biodiversity. Nature, 520, pp.45-50.

Olofsson, P. et al., 2014. Good practices for estimating area and assessing accuracy of land change. Remote Sensing of Environment, 148, pp.42-57.

Olofsson, P. et al., 2013. Making better use of accuracy data in land change studies: Estimating accuracy and area and quantifying uncertainty using strati fi ed estimation. Remote Sensing of Environment, 129, pp.122-131.

Pan, Y. et al., 2011. A large and persistent carbon sink in the world's forests. Science, 333(6045), pp.988-93.

Pipitone, C. et al., 2016. Data Service Platform for Sentinel-2 Surface Reflectance and Value-Added Products: System Use and Examples. Remote Sensing, 8, p.938.

Potapov, P. V. et al., 2012. Quantifying forest cover loss in Democratic Republic of the Congo, 2000-2010, with Landsat ETM+ data. Remote Sensing of Environment, 122, pp.106-116.

Pratihast, A.K. et al., 2014. Combining satellite data and community-based observations for forest monitoring. Forests, 5, pp.2464-2489.

Pratihast, A.K. et al., 2016. Design and implementation of an interactive webbased near real-time forest monitoring system. PLoS ONE, 11(3), p.e0150935.

Pratihast, A.K. et al., 2012. Mobile devices for community-based REDD+ monitoring: a case study for Central Vietnam. Sensors, 13(1), pp.21-38.

Quegan, S. \& Yu, J.J., 2001. Filtering of multichannel SAR images. IEEE 
Transactions on Geoscience and Remote Sensing, 39(11), pp.2373-2379.

Le Quéré, C. et al., 2009. Trends in the sources and sinks of carbon dioxide. Nature Geoscience, 2(12), pp.831-836.

Reiche, J., de Bruin, S., et al., 2015. A bayesian approach to combine Landsat and ALOS PALSAR time series for near real-time deforestation detection. Remote sensing, 7, pp.4973-4996.

Reiche, J., 2015. Combining SAR and optical satellite image time series for tropical forest monitoring $\mathrm{PhD}$ Thesis., Wageningen University, The Netherlands.

Reiche, J. et al., 2016. Combining satellite data for better tropical forest monitoring. Nature Climate Change, 6(2), pp.120-122.

Reiche, J. et al., 2013. Feature Level Fusion of Multi-Temporal ALOS PALSAR and Landsat Data for Mapping and Monitoring of Tropical Deforestation and Forest Degradation. IEEE Journal of Selected Topics in Applied Earth Observations and Remote Sensing, 6(5), pp.2159-2173.

Reiche, J., Verbesselt, J., et al., 2015. Fusing Landsat and SAR time series to detect deforestation in the tropics. Remote Sensing of Environment, 156, pp.276-293.

Reiche, J. et al., Improving near-real time deforestation monitoring in tropical dry forests by combining dense Sentinel-1 time series with Landsat and ALOS-2 PALSAR-2. Remote Sensing of Environment, In review.

Reichstein, M. et al., 2013. Climate extremes and the carbon cycle. Nature, 500(7462), pp.287-295.

Riano, D. et al., 2002. Assessment of vegetation regeneration after fire through multitemporal analysis of AVIRIS images in the Santa Monica Mountains. Remote Sensing of Environment, 79, pp.60-71.

Rosenqvist, A. et al., 2007. ALOS PALSAR: A Pathfinder Mission for GlobalScale Monitoring of the Environment. IEEE Transactions on Geoscience and Remote Sensing, 45(11), pp.3307-3316.

Rosenqvist, A. et al., 2014. Operational performance of the ALOS global systematic acquisition strategy and observation plans for ALOS-2 PALSAR-2. Remote Sensing of Environment, 155, pp.3-12.

Rouse, J.. et al., 1974. Monitoring vegetation systems in the Great Plains with ERTS. In 3rd Earth Resource Technology Satellite (ERTS) Symposium. pp. $48-62$.

Roy, D.P. et al., 2016. Characterization of Landsat-7 to Landsat-8 reflective wavelength and normalized difference vegetation index continuity. 
Remote Sensing of Environment, 185, pp.57-70.

Ryan, C.M. et al., 2012. Quantifying small-scale deforestation and forest degradation in African woodlands using radar imagery. Global Change Biology, 18(1), pp.243-257.

Saatchi, S. et al., 2012. Persistent effects of a severe drought on Amazonian forest canopy. PNAS, 110(2), pp.565-570.

van der Sanden, J.J., 1997. Radar remote sensing to support tropical forest management, Georgetown, Guyana: Tropenbos-Guyana Programme.

Sannier, C. et al., 2014. Using the regression estimator with Landsat data to estimate proportion forest cover and net proportion deforestation in Gabon. Remote Sensing of Environment, 151, pp.138-148.

Scarino, B.R. et al., 2016. A Web-Based Tool for Calculating Spectral Band Difference Adjustment Factors Derived From SCIAMACHY Hyperspectral Data. IEEE Transactions on Geoscience and Remote Sensing, 54(5), pp.2529-2542.

Schmitt, C.B. et al., 2010. Floristic diversity in fragmented Afromontane rainforests: Altitudinal variation and conservation importance. Applied Vegetation Science, 13, pp.291-304.

Schultz, M. et al., 2016. Performance of vegetation indices from Landsat time series in deforestation monitoring. International Journal of Applied Earth Observations and Geoinformation, 52, pp.318-327.

See, L., Fritz, S. \& Victoria, D.G., 2014. Beyond sharing Earth observations. Nature, 514, p.168.

Seneca Creek Associates, 2014. "Illegal" Logging and Global Wood Markets: The Competitive Impacts on the U.S. Wood Products Industry. Prepared for American Forest \& Paper Association by Seneca Creek Associates and Wood Resources International.

Setiawan, Y., Kustiyo, K. \& Darmawan, A., 2016. A simple method for developing near real-time nationwide forest monitoring for Indonesia using MODIS near- and shortwave infrared bands. Remote Sensing Letters, 7(4).

Sexton, J.O. et al., 2013. Global, 30-m resolution continuous fields of tree cover: Landsat-based rescaling of MODIS Vegetation Continuous Fields with lidar-based estimates of error. International Journal of Digital Earth, 6(5), pp.427-448.

Shimabukuro, Y.E. et al., 2006. Near real time detection of deforestation in the Brazilian Amazon using MODIS imagery. Revista Ambiente \& Água - An 
Interdisciplinary Journal of Applied Science, 1(1), pp.37-47.

Shimada, M. et al., 2014. New global forest/non-forest maps from ALOS PALSAR data (2007-2010). Remote Sensing of Environment, 155, pp.1331.

Souza, Jr, C. et al., 2013. Ten-Year Landsat Classification of Deforestation and Forest Degradation in the Brazilian Amazon. Remote Sensing, 5(11), pp.5493-5513.

Stehman, S. V., 2012. Impact of sample size allocation when using stratified random sampling to estimate accuracy and area of land-cover change. Remote Sensing Letters, 3(2), pp.111-120.

Stehman, S. V., 2009. Sampling designs for accuracy assessment of land cover. International Journal of Remote Sensing, 30(20), pp.5243-5272.

Steven, M.D. et al., 2003. Intercalibration of vegetation indices from different sensor systems. Remote Sensing of Environment, 88, pp.412-422.

Storey, J. et al., 2017. A note on the temporary misregistration of Landsat-8 Operational Land Imager (OLI) and Sentinel-2 Multi Spectral Instrument (MSI) imagery. Remote Sensing of Environment, 186, pp.121-122.

Strobl, C. et al., 2008. Conditional variable importance for random forests. BMC Bioinformatics, 9, p.307.

De Sy, V. et al., 2015. Land use patterns and related carbon losses following deforestation in South America. Environmental Research Letters, 10.

De Sy, V. et al., 2012. Synergies of multiple remote sensing data sources for REDD + monitoring. Current Opinion in Environmental Sustainability, 4, pp.696-706.

Thapa, R.B. et al., 2013. The tropical forest in south east Asia: Monitoring and scenario modeling using synthetic aperture radar data. Applied Geography, 41, pp.168-178.

Tian, F. et al., 2015. Evaluating temporal consistency of long-term global NDVI datasets for trend analysis. Remote Sensing of Environment, 163, pp.326340.

Torres, R. et al., 2012. GMES Sentinel-1 mission. Remote Sensing of Environment, 120, pp.9-24.

Tucker, C.J., 1979. Red and photographic infrared linear combinations for monitoring vegetation. Remote Sensing of Environment, 150, pp.127-150.

Tyukavina, A. et al., 2013. National-scale estimation of gross forest aboveground carbon loss : a case study of the Democratic Republic of the 
Congo. Environmental Research Letters, 8, p.14.

Uddstrom, M.J. \& Oien, N.A., 1999. On the use of high-resolution satellite data to describe the spatial and temporal variability of sea surface temperatures in the New Zealand region. Journal of Geophysical Research, 104(C9), pp.20729-20751.

Ulaby, T.F., Moore, R.K. \& Fung, A.K., 1986. Microwave remote sensing Active and Passive, Norwood, USA: Artech House.

UNFCCC, 2009. Cost of implementing methodologies and monitoring systems relating to estimates of emissions from deforestation and forest degradation, the assessment of carbon stocks and greenhouse gas emissions from changes in forest cover, and the enhancement of for. UNFCCC Secretary, Bonn, Germany.

Uwe, M.-W. et al., 2013. Sentinel-2 level 2A prototype processor : architecture , algorithms, and first results. In ESA Living Planet Symposium 2013, Edinburgh, UK. pp. 3-10.

Vaglio Laurin, G. et al., 2013. Optical and SAR sensor synergies for forest and land cover mapping in a tropical site in West Africa. International Journal of Applied Earth Observation and Geoinformation, 21, pp.7-16.

Venables, W.N. \& Ripley, B.D., 2002. MASS: modern applied statistics with S. Issues of Accuracy and Scale, p.868.

Verbesselt, J., Hyndman, R., Newnham, G., et al., 2010. Detecting trend and seasonal changes in satellite image time series. Remote Sensing of Environment, 114(1), pp.106-115.

Verbesselt, J., Hyndman, R., Zeileis, A., et al., 2010. Phenological change detection while accounting for abrupt and gradual trends in satellite image time series. Remote Sensing of Environment, 114(12), pp.29702980 .

Verbesselt, J., Zeileis, A. \& Herold, M., 2012. Near real-time disturbance detection using satellite image time series. Remote Sensing of Environment, 123, pp.98-108.

Verhegghen, A. et al., 2016. The Potential of Sentinel Satellites for Burnt Area Mapping and Monitoring in the Congo Basin Forests. Remote Sensing, 8, pp.1-22.

Vermote, E. et al., 2016. Preliminary analysis of the performance of the Landsat 8 / OLI land surface re fl ectance product. Remote Sensing of Environment, 185, pp.46-56.

Vermote, E., Justice, C.O. \& Bréon, F., 2009. Towards a Generalized Approach 
for Correction of the BRDF Effect in MODIS Directional Reflectances. IEEE Transactions on Geoscience and Remote Sensing, 47(3), pp.898-908.

Wegmüller, U. et al., 2016. Sentinel-1 support in the GAMMA software. Procedia Computer Science, 100, pp.1305-1312.

Werner, C. \& Strozzi, T., 2000. Gamma SAR and interferometric processing software. In Proceeedings of the 2000 ERS-ENVISAT Symposium. Gothenburg, Sweden.

Wheeler, D. et al., 2014. Satellite-based forest clearing detection in the Brazilian Amazon: FORMA, DETER, and PRODES, World Resources Institute.

Whittle, M. et al., 2012. Detection of tropical deforestation using ALOSPALSAR: A Sumatran case study. Remote Sensing of Environment, 124, pp.83-98.

Wilson, E., 1927. Probable inference, the law of succession, and statistical inference. Journal of the American Statistical Association, 22(158), pp.209-212.

Woodhouse, I., van der Sanden, J.J. \& Hoekman, D.H., 1999. Scatterometer observations of seasonal backscatter variation over tropical rain forest. IEEE Transactions on Geoscience and Remote Sensing, 37(2), pp.859-861.

Woodhouse, I.H., 2005. Introduction to Microwave Remote Sensing, CRC Press.

Wulder, M.A. et al., 2012. Opening the archive : How free data has enabled the science and monitoring promise of Landsat. Remote Sensing of Environment, 122, pp.2-10.

Wulder, M.A. \& Coops, N.C., 2013. Make Earth observations open access. Nature, 513, pp.2013-2014.

Xin, Q. et al., 2013. Toward near real-time monitoring of forest disturbance by fusion of MODIS and Landsat data. Remote Sensing of Environment, 135, pp.234-247.

Zeileis, A. et al., 2005. Monitoring structural change in dynamic econometric models. Journal of Applied Econometrics, 20(1), pp.99-121.

Zeileis, A., Shah, A. \& Patnaik, I., 2010. Testing , monitoring, and dating structural changes in exchange rate regimes. Computational Statistics and Data Analysis, 54(6), pp.1696-1706.

Zhang, H.K. \& Roy, D.P., 2016. Landsat 5 Thematic Mapper re fl ectance and NDVI 27-year time series inconsistencies due to satellite orbit change. Remote Sensing of Environment, 186, pp.217-233. 
Zhang, J., 2010. Multi-source remote sensing data fusion: status and trends. International Journal of Image and Data Fusion, 1(1), pp.5-24.

Zhu, Z. \& Woodcock, C.E., 2014a. Automated cloud, cloud shadow, and snow detection in multitemporal Landsat data: An algorithm designed speci fi cally for monitoring land cover change. Remote Sensing of Environment, 152, pp.217-234.

Zhu, Z. \& Woodcock, C.E., 2014b. Continuous change detection and classification of land cover using all available Landsat data. Remote Sensing of Environment, 144, pp.152-171.

Zhu, Z. \& Woodcock, C.E., 2012. Object-based cloud and cloud shadow detection in Landsat imagery. Remote Sensing of Environment, 118, pp.83-94.

Zhu, Z., Woodcock, C.E. \& Olofsson, P., 2012. Continuous monitoring of forest disturbance using all available Landsat imagery. Remote Sensing of Environment, 122, pp.75-91.

Zscheischler, J. et al., 2013. Detection and attribution of large spatiotemporal extreme events in Earth observation data. Ecological Informatics, 15, pp.66-73. 



\section{Summary}

Forests provide essential goods and services to humanity, but humaninduced forest disturbances have been on ongoing at alarming rates, undermining the capacity for forests to continue providing essential goods and services. In recent years, the understanding of the short-term and long-term impacts of deforesting and degrading forest ecosystems has improved, and global efforts to reduce forest loss are ongoing. However, in many parts of the globe, significant forest areas continue to be lost. To fully protect forest ecosystems efficiently, timely, reliable and location-specific information on new forest disturbances is needed. Frequent and large-area forest mapping and monitoring using satellite observations can provide timely and cost-effective information about new forest disturbances. However, there are still key weaknesses associated with existing forest monitoring systems. For example, the capacity for forest monitoring systems to detect new disturbances accurately and timely is often limited by persistent cloud cover and strong seasonal dynamics. Persistent cloud can be addressed by using observations from multiple satellite sensors, but satellite sensors often have inter-sensor differences which make integration of observations from multiple sensors challenging. Seasonality can be accounted for using a seasonal model, but image time series are often acquired at irregular intervals, making it difficult to properly account for seasonality. Furthermore, with existing forest monitoring systems, detecting subtle, low-magnitude disturbances remains challenging, and timely detection of forest disturbances is often accompanied by many false detections. The overall objective of this thesis is to improve forest change monitoring by addressing the key challenges which hinders accurate and timely detection of forest disturbances from satellite data. In the next paragraphs, I summarise how this thesis tackled some of the key challenges which hamper effective monitoring of forest disturbances using satellite observations.

Chapter 2 addresses the challenge of seasonality by developing a spatial normalisation approach that allows us to account for seasonality in irregular image time series when monitoring forest disturbances. In this chapter, I showed that reducing seasonality in image time series using spatial normalisation leads to timely detection of forest disturbances when compared to a seasonal model approach. With spatial normalisation, near real-time forest monitoring in dry forests, which has been challenging for many years, is now possible. Applying spatial normalisation in areas where evergreen and deciduous forests co-exist is however challenging. Therefore, further research is needed to improve the spatial normalisation approach to ensure that it is 
applicable to areas with a combination of different forest types. In particular, a spatial normalisation approach which is forest type-specifics is desirable. In this chapter, forest disturbances were detected by analysing single pixel-time series. Spatial information was only used to reduce seasonality.

Taking in account the fact that forest disturbances are spatio-temporal events, I investigated whether there is an added-value of combining both spatial and temporal information when monitoring forest disturbances from satellite image time series. To do this, I first developed a space-time change detection method that detects forest disturbances as extreme events in satellite data cubes (Chapter 3). I showed that, by combining spatial and temporal information, forest disturbances can still be detected reliably even with limited historical observations. Therefore, unlike approaches which detect forest disturbances by analysing single pixel- time series, the space-time approach does not require huge amount of historical images to be pre-processed when monitoring forest disturbances. I then evaluated the added-value of using space-time features when confirming forest disturbances (Chapter 4). I showed that using a set of space-time features to confirm forest disturbances enhance forest monitoring significantly by reducing false detections without compromising temporal accuracy. With space-time features, the discrimination of forest disturbances from false detections is no longer based on temporal information only, hence providing opportunity to also detect low-magnitude disturbances with high confidence. Based on the analysis for conditional variable importance, I showed that features which are computed using both spatial and temporal information were the most important predictors of forest disturbances, thus enforcing the view that forest disturbances should be treated as spatio-temporal in order to improve forest change monitoring.

In Chapter $2-4$, forest disturbances where detected from medium resolution Landsat time series. Yet, recent studies showed that small-scale forest disturbances are often omitted when using Landsat time series. In Chapter 5, I investigated whether detection of small-scale forest disturbances can be improved by using the $10 \mathrm{~m}$ resolution time series from recently launched Sentinel-2 sensor. I also investigated whether the spatial normalisation approach developed in Chapter 2 can be used to reduce intersensor differences in multi-sensor optical time series. I showed that the $10 \mathrm{~m}$ resolution Sentinel-2 time series improves the detection of small-scale forest disturbances when compared to $30 \mathrm{~m}$ resolution. However, the $10 \mathrm{~m}$ resolution does not supersede the importance of frequent satellite observations when monitoring forest disturbances. I also showed that spatial normalisation approach developed in Chapter 2 can reduce inter-sensor differences in multisensor optical time series significantly to generate temporally consistent time series suitable for forest change detection. Spatial normalisation does not completely remove inter-sensor differences, but the differences are significantly reduced. 
Monitoring of forest disturbances is increasingly done using a combination of Synthetic Aperture Radar (SAR) and optical time series. Therefore, Chapter 6 investigated whether the spatial normalisation approach developed in Chapter 2 can also reduce seasonal variations in SAR time series to facilitate the integration of SAR-optical time series for forest monitoring in dry tropical forests. This Chapter demonstrated that seasonal variations in SAR time series can also be reduced through spatial normalisation. As a result, observations from SAR and optical time series were combined to improve near real-time forest change detection in dry tropical forest. In Chapter 7, it is demonstrated that spatial normalisation has potential to also reduce intersensor differences in SAR-optical time series, resulting into temporally consistent SAR-optical time series.

In conclusion, this thesis developed a space-time forest monitoring framework that addresses some key challenges affecting satellite-based forest monitoring. In particular, new methods that allow for timely and accurate detection of forest disturbances using observations from multiple satellites were developed. Overall, the methods developed in this research contribute to our capacity to accurately and timely detect forest disturbances in both dry and humid forests. 



\section{Acknowledgements}

Completing a $\mathrm{PhD}$ requires a great deal of self-motivation, but it is the depth and consistency of support you receive from the people around you that gets you to the finishing line. As I arrive at the finishing line of my $\mathrm{PhD}, \mathrm{I}$ would like to thank the people who gave me deep and consistent support during the last four years of my PhD journey. The chronology in which I thank them does not reflect the superiority of the support.

First I would like to thank Martin - my promotor. Martin, it was in August - the summer of 2012 (Dutch summer) - I was just winding up my stay in The Netherlands after my MSc studies hence my mind was fully set for Namibia - when I heard that you were looking for me. The news of you looking for me made me wonder a lot about what could possibly trigger you to look for me. On my way to your office, to hear why you were looking for me, many thoughts crossed my mind, but a PhD offer was not part of such thoughts in any way. Upon arrival in your office, you did not hesitate to let me know that there is a possibility of a $\mathrm{PhD}$ offer in your research group. With this news, you sent my mind in the "forest of confusion" for some few minutes. Remember, my mind was fully set for Namibia -not for a prolonged stay in The Netherlands. Such confusion was inevitable because, suddenly, I had to decide at that point in time whether to accept the offer or to stick to my plans of going permanently to Namibia. I do not remember much of what I said to you as a package of my response, but at least I remember clearly telling you that I would take the offer. After few months of holiday and preparations in Namibia, I came back to The Netherlands in February - the winter of 2013 - to begin with a new journey - a PhD journey in your research group. Martin, from there on, you took me under your wings, helping me find my path, and sometimes confusing me with your research ideas during our $\mathrm{PhD}$ meetings. You gave me endless opportunities to grow and the "ocean of freedom" to explore and implement my research ideas. Martin, it is the depth and consistency of the support you gave me in the last four years that makes me deeply thankful to you. You have been a fantastic promotor.

In 2011, prior to starting my $\mathrm{PhD}$ journey and after completing the coursework of my MSc in Wageningen, I popped into the office of Jan Verbesselt, to discuss the possibility of him becoming the supervisor of my MSc thesis. I had my own research idea that I wanted to pursue. More specifically, I wanted to use earth observation data to investigate the effect of climate variability on the vegetation dynamics in Etosha National Park, Namibia. Immediately after I finished explaining my idea, Jan asked me if I had validation data. My answer was a straight "no validation data". In a flash of a 
second, Jan suggested that may be I should work on the analysis of phenological changes instead, but the study area would be Europe where validation data were freely available. Not fully convinced by this idea, I somewhat agreed to explore that possibility. Again, in a flash of a second, Jan started showing me his research work on near real-time change monitoring, more specifically the idea of BFAST-Monitor. Jan, I still do not know why you were showing me that work, but I at least know that, in Chapter 2 of my PhD thesis, I used the algorithm that you were developing. Sticking with the idea of phenological changes, together with Gerbert Roerink, you become the supervisor of my MSc thesis and you constantly guided me during the MSc thesis. Eventually, we published a peer-reviewed journal article from the MSc work - although on a later stage. Fate had it; you become my co-promotor for my $\mathrm{PhD}$. Of course this was not a surprise to me. I knew from the beginning when Martin made the offer to me that I would work with you. Just few days after settling back in Wageningen, we had our first meeting - to iron out the $\mathrm{PhD}$ proposal. After four months of hard-work, the proposal was ready. The rest is history. Then the most difficult part of $\mathrm{PhD}$ journey began - the implementation of the research ideas crafted in the research proposal. Together with Martin, you helped me find my way. You were constantly present when I needed your help or when I was completely lost in the scientific world. I remember vividly when you invited me for a "scientific walk" around the lake behind Lumen Building in Wageningen. I knew you were probably worried that may be I was somehow stuck, and you wanted to help me find my way. Jan, you motivated me when my morale was ever low. You would call and ask if all was well with me. You always had my back. You would not give up on me even when I did not seem fit to be a PhD. Jan, there were many moments I paused and wondered whether I really deserved all that deep and consistent support you gave me. What I however know for sure is that I am deeply and forever indebted to you. You were a critical force that got me to the finishing line. Thank you, Jan! I look forward to many collaborations with you in the future.

During my PhD journey, the steam of administrative work was always on. Dealing with the steam of administrative work during a $\mathrm{PhD}$ journey is tough because oftentimes the energy reserve is emptied by the "scientific engine". Surprisingly, I survived that steam of administrative work. This was mainly because of Antoinette and Truus. At all time, Antoinette and Truus were ever available and ready to assist whenever I needed to have administrative work sorted out. Antoinette and Truus, it is because of your unwavering support that you rendered to me during my $\mathrm{PhD}$ journey that I will forever be grateful to you. To this I say: dank je!!

Without your colleagues, a PhD journey can be a lonely adventure filled with a multitude of scientific barriers, which can send you into distress. In my case, I was lucky to escape this trap of distress. I had a "sea of good colleagues" that helped me escape this trap. My colleagues shielded me from academic 
loneliness, and were a great source of comfort when the going got tough. Our academic discussions formed a great source of ideas that greatly enriched my $\mathrm{PhD}$ research. The collegiality of my colleagues was essentially a mantle of hope during my $\mathrm{PhD}$ journey. It is for this reason that I shall forever be grateful to Sytze, Ben, Eskender, Mathieu, Sarah, Benjamin, Kim, Johannes R., Jose, Nandika, Michi, Giulia, Richard, Niki, Loïc, Arun, Patric, Roberto, Brice, Arnold, Erika, Kalkidan, Astrid, Yang, Lammert, Simon, Valerio, Peter, Rosa Maria, Harm, Marcio, Ron, John, Alvaro, Juha, Arend, Willy, Qijun, Johannes E., Lukasz, Maria, Marston, Aldo, Jan C., Roland, Frans, Philip, Anne, Konstantin, Sabina, Cristina, Jalal, Marcelo, Danaë, Joao, Daniela and Corne.

A "sea of good colleagues", can provide you with critical comfort and necessary support during your $\mathrm{PhD}$ journey, but such comfort and support are inadequate in the absence of good friends.

I have a "mountain of friends" that helped me get into, and through, my $\mathrm{PhD}$ life. Jat, Joseph and Veronica, in the last four years, you took up a burden of responsibilities which was never yours. Yet, you carried such responsibilities on your shoulders with sheer excellence. Please, tell me - how do I thank you when the burden was too heavy? You know very well what I am referring to here. Nevertheless, I am deeply grateful to you. When I had so many doubts on whether I should really return to The Netherlands for a $\mathrm{PhD}$, it was Malakia (Embodi), Jossy and Martin Hipondoka who produced words of wisdom to brutally encounter my doubts. Malakia, Jossy and Martin Hipondoka, your words basically got me into the plane to The Netherlands. Today, I would like to thank you for your wise words. With my doubts clashed, I landed back in Wageningen on the $5^{\text {th }}$ of February 2013. But, I was now confronted by a risk the risk of getting homesick. Fortunately, during my stay in Wageningen, I was lucky to be graced by the friendship of Melvin, Mkhize, Koki, Matthew, Judie, Albertina, Valerie, Fecilidade, Cecario, Eliana, Christie, Precious, Wallou, Simone, Yaqing, Bongani, Vincent, and Sebastine (my Oga). Your friendship was the tranquiliser of my homesickness during my time in Wageningen. Thank you! Back home, the tentacles of friendship never ceased to reach for me. Far away from home yet I never felt the absence of the warmth of friendship from Chichi, Titus, Maria, Kotokeni, Muni, Imms, Katox, Lylio, Mesty, Verena, Ottilie Mwazi, Grace, Paulina, Naveuye, Nanhapo, Hilma, Uazu, Alex, Sphumtile, Phile, Pauline, Tuma, Six, Samora, Joe Hangula, and Joe Namhindo. May your tentacles of friendship continue reaching for me to provide warmth of friendship to me my dear friends. Indeed, my last months of the $\mathrm{PhD}$ journey were intense and unforgiving, and sometimes were mentally draining. But, such intense and unforgiving period was neutralised by the warmth of friendship from Ndeshie. Our lengthy conversations - conversations between strangers, yet friends, helped me reach the finishing line. 
A combination of a "sea of good colleagues" and a "mountain of friends" was indeed critical to the completion of my $\mathrm{PhD}$ journey, but it was the support I received from my family and the sacrifice my family made during my absence in their mix that kept me walking forward during my $\mathrm{PhD}$ journey. This unwavering family support originated from my parents (Namukwiyu and Hendrina), my siblings (Rebeka, Isreal, Elise, Tuulongelwa, Tuhafeni, Justina, Kaleinasho, Ndiilokelwa, Shidute, Kauna, Simon, Ndiiwana and Pomwene), nieces (Ndeshi, Maria), and cousins (Pandulo, Weyulu). I know, my prolonged absence in your mix literally denied you the presence of a son, brother and a cousin and a relative. I hope you will one day be proud that your son, a brother, a cousin and a relative - who has been away from home pursuing a $\mathrm{PhD}$ journey - is now coming home. Namukwiyu- my father-it was September 2012, I had just returned home from The Netherlands after two years of MSc studies. You were so happy to have me back home. Then, I broke the news of me going back to The Netherlands, this time around for fours to pursue a PhD. I still remember vividly your 2 -seconds facial expression. I knew you were basically saying I must be crazy to do that, and of course you were worried given the length of the period I would be away. Despite that expression, you nonetheless wished me luck in my endeavour. This is characteristic of you -to provide support and to be un-limiting parent. Daddy, fours is gone -and I am coming home. For now all I want to say is: thank you for your ever-present support and for being unlimiting daddy. To my late mom, Lydia, although you have been gone for about 22 years now, as I arrive at the finishing line, I wish you could know that your boy that you sent to school about 27 years ago is now coming home after 22 years in school. Nevertheless, I just want to let your spirit know that your message of hard-work, focus and self-discipline that you delivered to me 27 years ago, when I entered the door of school for the first time in 1990, has taken me through to the end.

Indeed, there are many other people who helped me immensely before or during my PhD journey, whose names I did not mention here. To them I say, your support to me is highly appreciated.

Finally, I admit, it is so difficult to fully express sufficient gratitude to a bunch of people who have been extremely good to you. I could not thank you all sufficiently, but please find solace in knowing that your support to me did not end in vain.

To you all, I say: Thank you!! Tangi unene!! Dank je!!

Eliakim

Wageningen, 17 July 2017 


\section{Publications}

Peer-reviewed journals:

Hamunyela, E., Reiche, J., Verbesselt, J., \& Herold, M. (2017). Using spacetime features to improve detection of forest disturbances from Landsat time series. Remote Sensing, 9 (6), 515, DOI: 10.3390/rs9060515

Hamunyela, E., Verbesselt, J., de Bruin, S., \& Herold, M. (2016). Monitoring Deforestation at Sub-Annual Scales as Extreme Events in Landsat Data Cubes. Remote Sensing , 8(8), 651, DOI: 10.3390/rs808065

Hamunyela, E., Verbesselt, J. \& Herold, M. (2016). Using spatial context to improve early detection of deforestation from Landsat time series. Remote Sensing of Environment, 172, 126-138, DOI: 10.1016/j.rse.2015.11.006

Hamunyela, E., Verbesselt, J., Roerink, G. \& Herold, M. (2013). Trends in Spring Phenology of Western European Deciduous Forests. Remote Sensing, 5, 6159-6179, DOI:10.3390/rs5126159

Manuscripts in preparation/review:

Hamunyela, E., Reiche, J., Verbesselt, J., Tsendbazar, N.E., \& Herold, M. Combining Sentinel-2 and Landsat time series for small-scale forest change monitoring. In prep.

Lu, M., Hamunyela, E., Pebesma, E. \& Verbesselt, J. Dimension reduction of multi-spectral 1 satellite image time series to improve deforestation monitoring. Remote Sensing. In review.

Reiche, J., Hamunyela, E., Verbesselt, J., Hoekman, D., \& Herold, M. Improving near-real time deforestation monitoring in tropical dry forests by combining dense Sentinel-1 time series with Landsat and ALOS-2 PALSAR-2. Remote Sensing of Environment. In review.

Beza, E., Hamunyela, E., Reidsma, P., Belay, M. M, \& Kooistra, L. Remote sensing and crowdsourcing for estimating and explaining yields: sesame in Ethiopia. In prep.

Conferences proceedings/talks and contributions:

Lu, M. \& Hamunyela, E. (2016). On-line change monitoring with transformed multi-spectral time series, a study case in tropical forest. The 
International Archives of the Photogrammetry, Remote Sensing and Spatial Information Sciences, Volume XLI-B7, 2016 XXIII ISPRS Congress, 12-19 July 2016, Prague, Czech Republic . DOI: 10.5194/isprsarchives-XLI-B7-987-2016

Hamunyela, E., Verbesselt, J., de Bruin, S., Herold, M. Spatio-temporal Monitoring of Deforestation in Dry Forests using Satellite Image Time Series. ESA Living Planet Symposium 2016, Prague, Czech Republic, 9-13 May 2016.

Verbesselt, J. DeVries, B., Reiche, J., Dutrieux, L, P., Hamunyela, E., Herold, M. Detecting, monitoring and charactering ecosystem change using multiple satellite sensor image time series. European Space Agency Living Planet Symposium 2016, Prague, Czech Republic, 9-13 May 2016.

Hamunyela, E., Verbesselt, J., Herold, M. Improving near real-time mapping of forest disturbances from Landsat time series using spatial context. 12th Regional Workshops on Forest Monitoring GEO GFOI Early Warning Systems for deforestation. San Jose Dos Campos, Brazil, January 19-23, 2014

Dutrieux, L., P., Hamunyela, E., Verbesselt, J., Kooistra, L., Herold, M. Spatio-temporal break detection for deforestation monitoring using Landsat and MODIS image time series. International Conference "Global Vegetation Monitoring and Modeling” (GV2M) 2014. Avignon, France, 03 -07 February 2014.

Hamunyela, E., Verbesselt, J., Schultz, M., Penndorf, A., Frotscher, K., Herold, M., Reiche, J., DeVries, B. , Dutrieux, L., P., Calders, K. Tracking forest cover change using Landsat \& Rapid Eye towards S2. Sentinel-2 for Science Workshop, Frascati, Italy, 20 - 22 May 2014. 


\section{Short biography}

Eliakim Hamunyela was born on 28 May 1984 in Omangela village, Namibia. From 1990 to 1999, he attended his primary education at Omundudu Combined School, about $1.5 \mathrm{~km}$ from his homestead. Growing up as a typical village boy, Eliakim enjoyed balancing his time between school work and looking after cattle and goats, ploughing the cropfield and hunting for birds using a catapult. These activities provided him with different but complementary perspectives about life, thus helping him to always graduate amongst the best students in his class during his primary education. In 2000, Eliakim moved to Eengedjo Secondary School, a boarding school, to pursue his secondary education. From 2000 to 2002, while at secondary school, Eliakim continued to perform well in school, earning him several awards, including being the overall best learner in Eengedjo Secondary School in 2000. For his final national secondary examination, he was awarded a prize of the best learners in Physical Science and Agriculture in Ohangwena region.

With sponsorship from the national power utility, NamPower, Eliakim moved to Windhoek, Namibia, in 2003 to pursue a four year BSc in Environmental Biology and Geography at the University of Namibia.

After graduating from the University of Namibia in 2007, Eliakim joined the Central Bureau of Statistics, National Planning Commission, Namibia, as GIS Analyst based in Otjozondjupa Region. He was then promoted to the position of a senior GIS Analyst in 2010, thus moving back to Windhoek, to head the Census Mapping Project. The project had a goal of creating a national dwelling frame, to improve the population and housing census as wells as the local and regional development planning. In September of the same year, Eliakim was awarded a scholarship through the Netherlands Fellowship Programme to pursue a MSc. in Geoinformation Science at Wageningen University, The Netherlands. After completing his MSc studies, he was offered an opportunity to pursue a $\mathrm{PhD}$ research at the Laboratory of Geo-information Science and Remote Sensing at Wageningen University, under the supervision of Prof Martin Herold and Dr Jan Verbesselt. His PhD research work culminated into this thesis. His $\mathrm{PhD}$ research provided him with many opportunities to travel across the globe, including a visit to Google headquarters, Mountain View, USA, in 2014 as a visiting scientist.

Eliakim's current research interests are related to near real-time environmental monitoring using satellite observations, time series analysis and fusion of earth observation data for environmental monitoring. He is the creator of the STEF package (https://github.com/hamun001/STEF) - an open source $\mathrm{R}$ package for space-time forest change monitoring. 



\section{PE\&RC Training and Education Statement}

With the training and education activities listed below the $\mathrm{PhD}$ candidate has complied with the requirements set by the C.T. de Wit Graduate School for Production Ecology and Resource Conservation (PE\&RC) which comprises of a minimum total of 32 ECTS (= 22 weeks of activities)

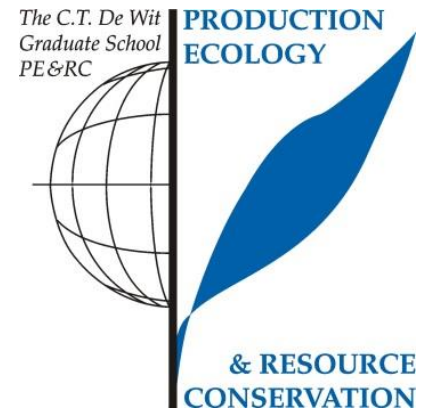

Review of literature (6 ECTS)

- Near real-time monitoring of forest change dynamics using satellite observations

\section{Writing of project proposal (4.5 ECTS)}

- Spatio-temporal approaches for satellite monitoring of global forest change dynamics

Post-graduate courses (8.6 ECTS)

- Multivariate analysis; PE\&RC (2013)

- Sampling in Space and Time for Survey and Monitoring of Natural Resources; PE\&RC (2013)

- $\quad$ Bayesian Statistics; PE\&RC (2013)

- $\quad$ Mixed Linear Models; PE\&RC (2013)

- $\quad$ Linear Models; PE\&RC (2013)

- Generalised Linear Models; PE\&RC (2014)

- Geostatistics; PE\&RC and SENSE (2015)

- $\quad$ Structural equation modelling; PE\&RC and WASS (2015)

Laboratory training and working visits (1.2 ECTS)

- Implementing BFAST algorithms in Google Earth Engine; Google, Mountain View, California

Invited review of (unpublished) journal manuscript (2 ECTS)

- Global Change Biology: satellite-derived phenological changes (2014)

- Remote Sensing: detection of spatio-temporal changes (2015)

Competence strengthening / skills courses (1.8 ECTS)

- Technique for writing and presenting a scientific paper; WGS (2014)

- Interpersonal communication for PhD students; WGS (2016)

PE\&RC Annual meetings, seminars and the PE\&RC weekend (1.8 ECTS)

- $\quad$ PE\&RC First year weekend (2013)

- PE\&RC Day (2014)

- $\quad$ PE\&RC Midterm weekend (2015) 
Discussion groups / local seminars / other scientific meetings (6 ECTS)

- $\quad$ R Users meeting (2013-2014)

- Remote sensing thematic group meeting (2013-2016)

International symposia, workshops and conferences (5 ECTS)

- $12^{\text {th }}$ Regional Workshops on Forest Monitoring GEO GFOI Early Warning Systems for deforestation: improving near real-time mapping of forest disturbances from Landsat time series using spatial context; San Jose Dos Campos, Brazil (2014)

- ESA Living Planet Symposium: spatio-temporal monitoring of deforestation in dry forests using satellite image time series; Prague, Czech Republic (2016)

Supervision of MSc students

- Bongani Ngwenyama: comparing the performance of structural change approaches

- Amalia Castro Gómez: improving forest monitoring: combining temporal and spatial information 

This work was supported financially by Google through Deforestation Monitoring project (grant agreement 5120906), the European Space Agency through ForMoSa project - Forest Degradation Monitoring with Satellite Data Project (grant agreement 5160957022) and by the European Union through The Role of Biodiversity in Climate Change Mitigation project (FP7/2007-2013; grant agreement 283093).

Cover design:

Initial design by Malakia (Embodi) Haimbangu

Redesigned by Ruben Stoel of Proefschriftmaken | www.proefschriftmaken.nl 